\title{
Phenomena at the border between quantum physics and general relativity
}

by

\author{
Valentina Baccetti
}

\author{
A thesis \\ submitted to the Victoria University of Wellington \\ in fulfilment of the \\ requirements for the degree of \\ Doctor of Philosophy
}

in the School of Mathematics, Statistics and Operations Research.

Victoria University of Wellington 2014 



\begin{abstract}
In this thesis we shall present a collection of research results about phenomena that lie at the interface between quantum physics and general relativity. The motivation behind our research work is to find alternative ways to tackle the problem of a quantum theory of/for gravitation.

In the general introduction, we shall briefly recall some of the characteristics of the well-established approaches to this problem that have been developed since the beginning of the middle of the last century. Afterward we shall illustrate why one would like to engage in alternative paths to better understand the problem of a quantum theory of/for gravitation, and the extent to which they will be able to shed some light into this problem.

In the first part of the thesis, we shall focus on formulating physics without Lorentz invariance. In the introduction to this part we shall describe the motivations that are behind such a possible choice, such as the possibility that the physics at energies near Planck regime may violate Lorentz symmetry. In the following part we shall first consider a minimalist way of breaking Lorentz invariance by renouncing the relativity principle, that corresponds to the introduction of a preferred frame, the aether frame. In this case we shall look at the transformations between a generic inertial frame and the aether frame still requiring the transformations to be linear. The second step is to establish the transformations for the energy and momentum in order to define some dynamics and design possible experiments to test such assumptions. As an application we shall present two compelling models that minimally break Lorentz invariance, the first one only in the energy-momentum sector, the second one in the transformation between inertial frames. Following along the line of physics without Lorentz invariance, we shall next explore some threshold theorems in both scattering and decay processes by considering only the existence of some energy momentum relation $E(p)$, without making any further assumption. We shall see that quite a lot can be said and that 3-momenta can behave in a complicated and counter-intuitive manner.

In the second part of the thesis we shall address the thermodynamics of spacetime and the important role played by entropy. In the introduction we shall outline the idea of induced gravity, which is the motivation behind this possible interpre-
\end{abstract}


tation of general relativity as a mean field theory of some underlying microscopic degrees of freedom. In the next chapter we shall partially review Jacobson's thermodynamic derivation of the Einstein equations and generalise it to a generic birfucate null surface. The interesting result we shall see is that, given the construction of the thermodynamic system via some virtual constantly accelerating observers, we can assign a "virtual" definition of Clausius entropy to essentially arbitrary causal horizons. To conclude this part we shall present some of the mathematical properties of entropy. In particular we shall focus on the simpler case of single-channel Shannon entropy and study under which conditions it is infinite, even though the probability distribution is normalisable.

In the last part, we shall describe a proposal for a space-base experiment to test the effects of acceleration and gravity of quantum physics. In principle, the results of such an experiment could shed some light on fundamental questions about the overlap of quantum theory and general relativity; at the same time, they may enable experimentalists interested to implement quantum communication into space based technology, to correct adverse gravitational effects.

We conclude with a brief discussion of lessons learned from these different approaches. 
(C)2014, Valentina Baccetti 
The journey is long and the path is pathless and one has to be alone.

There is no map and no one to guide.

But there is no alternative

One cannot escape it, one cannot evade it.

One has to go on the journey.

The goal seems impossible but the urge to go on it is intrinsic.

The need is deep in the soul.

Really, you are the urge, you are the need and consciousness cannot be otherwise because of this challenge and because of this adventure.

So do not waste time - begin.

Do not calculate - begin.

Do not hesitate - begin.

Do not look back - begin

And always remember old Lao Tzu's words:

A tree that takes both arms to encircle grows from a tiny rootlet.

A many-storied pagoda is built by placing one brick upon another brick.

A journey of three thousand miles is begun by a single step.

OSHO - A Cup of Tea 


\section{Acknowledgments}

And here it comes, the moment to thank all the people who helped me to achieve this great goal that a $\mathrm{PhD}$ is. First things first, I would like to thank Matt, my supervisor, for all the support and guidance you have given me in the last three years. I have truly appreciated all the scientific discussions we have had and all the work we have done together. I hope I have been able to grab even the tiniest amount of your passion for science and for research, and your huge knowledge. I am profoundly grateful to my officemates, Prado and Kyle. We have shared part of this journey together, with its ups and downs. I wish you good luck for your future careers, but knowing you guys, I am sure your future will be bright. Another key ingredient in the completion of this thesis is the RQI group in Nottingham. Ivette, Carlos, Nico, Ant, Gerardo, Mehdi, David, Jason, Giannis, Angela, thank you guys for having welcomed me in your group of "metrology boys and space girls" so quickly. I have learned so much about physics, team work and friendship in the six months I have spent with you. I hope I can visit you soon again. I also want to thank Nicolas Menicucci for all the scientific discussions we have had, for all the physics you have taught me, and for the support you gave me when I needed it more. I want to thank all the staff members at the School of Mathematics, Statistics and Operations Research at Vic, in particular my secondary supervisor Mark McGuinness; Prema, Kelsey, Ginny and Tania, and all the members of the school office, your help was always more than appreciated and your smily faces made several of my mornings at Uni. I also want to thank Steven Archer for the nice support you have given me for the tutoring and marking, and Radek for being such a nice guy.

I also want to thank Vladimir, Xenia and Sviatoslav Pestov, for the Irene Pestov's Memorial Scholarship, set up in memory of their late wife and mother Irene Pestov, of which I am a humble recipient.

And now let us go to all the friends who have supported me with their company and laughs during these three long years. The biggest thank you goes to Filippo and Giulia S., for basically being my family here in Wellington. All the movie nights we 
have had together, the holiday in Coromandel under the tropical typhoon with your amazing families, the Weta party, the Sundays at the farmers market, all the simple moments we have shared, have been incredible for me. I am more than glad to see you both succeeding in your dreams. Also I need to thank Patato and Kali; without you little silly creatures, life would not be as entertaining at Rodrigo Rd. A big hug and thank you go to Elisa and Raphael, with whom I have had delicious dinners and interesting conversations based on physics and rum (at some stage more rum than physics). Thank you Elisa for making me laugh with your blog every time I needed. Also a big thank you to Cristina, Lorena, Marcello, Sonja, Giulia M. and Rachele, for all the time we have spent together, and for reminding me that there is a world outside; Marcello for the beautiful experience on the Milford track and the motto "stai sereno". Roberto for the amazing fresh pasta and pizza you make at Merkato Fresh and for the spirit of Rome you brought to Wellington, every time I visit you I feel at home. Marco Z., I am very glad to see you pursuing your dreams, and I am more than thrilled for you, I hope I can help you find some interesting science that you can show in one of your documentaries. Daniel and Juan, my friends of many chats and coffees, your latin warmth made me feel like at home, your friendship is very dear to me.

Lia, for being my family in Nottingham, I really appreciated all the times we went to Tesco around midnight, just because we wanted. The time we spent together was some of the best in my life. I hope you have enjoyed it too. Manuel, Kai, Wilhelm, Giannis, Benito, Anna, Rowan, Gael, Richard, Nico, Hugo, Giulia G., Silvio, Simona, Michalis, Sid, Sara, Ant, Luis, Cristiano, Daniele and Federica for all the parties, improvised dinners and picnics in Wollaton park. You made me spend a phenomenal summer in the UK, I really want to meet you all at some stage and I deeply miss you. Do not worry Giannis, I will start Salsa classes as soon as I finish this thesis. (Thank you Daniele for your pressure cooker, the cast iron pot, and the bike, I think I have lost count of all the things you lent us.) A big thank you for all my rock climbing friends in Nottingham and in Wellington. Anna, Rowan, Lia, Manuel, Gael, Daniel, Richard, Michalis, Wilhelm, Ellie, Gemma, Gabriela, Francesco, Achim, Romain, Fabrizio, Marco and Anya, for being awesome fellows in my attempts to defy gravity also in the outdoor. I have loved our trips to the gym, to the Peak District, and Turakirae Head, I would love to climb with all of you one day. Thank you for the great example and the inspiration. And Vacho, for our silly conversations on Skype, thank you for all the times you were there for me, and for contradicting each and every single thing I said (and the fantastic music you have given me in these years). I'm very grateful to my very dear friends and mentors 
Lorenzo Sindoni and Gabriele Palombo. The endless conversations we have had, have always been a source of inspiration to me and have deeply shaped my path and thoughts.

Lastly the most important people in my life. Mum and dad, thank you for all your support in these years that I have been so far away from you. I would not be here without all the things you have taught me, without all the love you have given me. I hope I can show you the New Zealand beautiful landscape and rough sea one day. Also I need to thank my brothers, Claudio and Fabrizio, for being ... awesome brothers, for sharing several passions with me, and for all the times you had made me laugh. And a big thank you to your wife Bea and fiancé Veronica. This thesis would not be here without you Steve, your unconditional love helped me going through the worst moments of my life. Your example as a great scientist has helped me to push my boundaries beyond anything I have thought possible. Thank you for believing in me and leaving me free to grow as a person. You know where your place in my heart is. I also need to thank your family for their support even from such a distance. Merrilee, I know you are going through the same epic endeavour as me, writing a $\mathrm{PhD}$ thesis. I know you will do great. 


\section{Contents}

\begin{tabular}{lll}
\hline & Introduction & 1
\end{tabular}

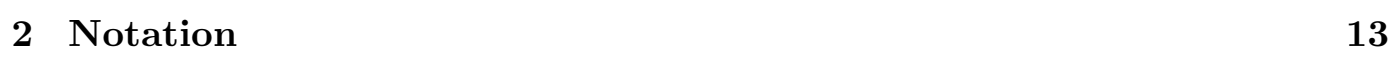

I Physics without Lorentz invariance 15

\begin{tabular}{|lll}
3 & An overview of Lorentz symmetry breaking & 17
\end{tabular}

4 Inertial frames without the relativity principle $\quad 21$

4.1 Introduction . . . . . . . . . . . . . . . . . . . . . . . . 21

4.2 General transformations between inertial frames . . . . . . . . . . . 23

4.2 .1 Definition of an inertial frame . . . . . . . . . . . . 23

$4.2 .2 \quad$ Argument for linearity . . . . . . . . . . . . . . . . . . 24

$4.2 .3 \quad$ General representation of inertial transformations . . . . . . . 26

4.2 .4 Aether frame and moving frame . . . . . . . . . . 28

4.2 .5 Transformation of 3 -velocity . . . . . . . . . . . . . . . 30

4.2 .6 Groupoid/pseudogroup structure . . . . . . . . . . . . . . . . . . . . . . . . 31

4.3 Transformations of energy and momentum . . . . . . . . . . . . 33

$4.3 .1 \quad$ Defining energy and momentum . . . . . . . . . . . 33

4.3 .2 Affine versus linear transformations . . . . . . . . . . . 35

4.3 .3 Summary (Energy-momentum) $\ldots \ldots \ldots \ldots$

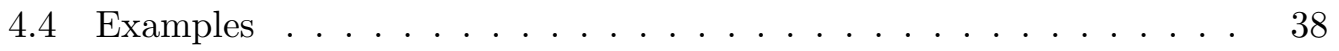

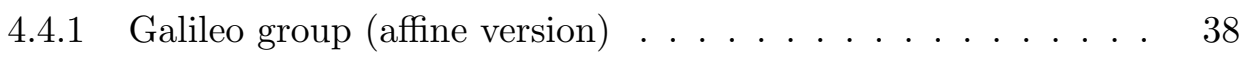

4.4 .2 Lorentz group (linear version) . . . . . . . . . . . . . . . . 39

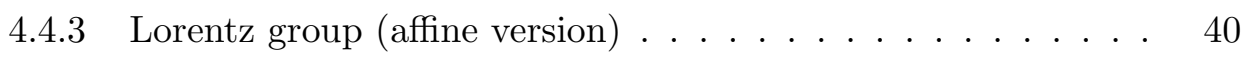

$4.4 .4 \quad$ Galileo group (linear version) . . . . . . . . . . . . . . . . . . 42

4.4 .5 Considerations regarding affine versus linear. . . . . . . . . . 43

4.5 On-shell relations for energy and momentum . . . . . . . . . . . 43 
$4.5 .1 \quad$ Rest energy without the relativity principle . . . . . . . . . . 43

4.5 .2 Invariant rest energy without the relativity principle . . . . . 45

$4.5 .3 \quad$ First minimalist Lorentz-violating model . . . . . . . . . . . . 46

4.5 .4 Considerations regarding on-shell energy-momentum . . . . . 48

4.6 Adding more constraints . . . . . . . . . . . . . . . . . . . . . . . 49

$4.6 .1 \quad$ Linearity plus isotropy . . . . . . . . . . . . . . . . . . . 49

4.6 .2 Linearity plus isotropy plus reciprocity . . . . . . . . . . 52

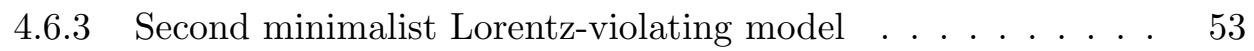

4.6 .4 Linearity plus isotropy plus reciprocity plus relativity . . . . 55

4.7 Conclusions regarding the relativity principle $\ldots \ldots \ldots$. . . . . . 57

5 Lorentz violating kinematics: Threshold theorems 59

5.1 Introduction . . . . . . . . . . . . . . . . . . . 59

5.2 General background $\ldots \ldots \ldots$. . . . . . . . . . . . . 62

5.3 Cautionary comments $\ldots \ldots \ldots \ldots$. . . . . . . . . . . 63

5.4 Decay thresholds . . . . . . . . . . . . . . . . . . . . 65

$5.4 .1 \quad$ Kinematically allowed region $\ldots \ldots \ldots \ldots$. . . . . . 65

5.4 .2 Thresholds in momentum space . . . . . . . . . . . . . 67

$5.4 .3 \quad$ Lagrange multiplier techniques . . . . . . . . . . . . . . 74

5.4 .4 Thresholds in terms of energy . . . . . . . . . . . 76

5.4 .5 Asymmetric thresholds . . . . . . . . . . . . . . . 77

5.4 .6 Some examples . . . . . . . . . . . . . . . . . . . 78

5.4 .7 Monotonicity . . . . . . . . . . . . . . . . . 80

5.4 .8 Isotropy $\ldots \ldots \ldots \ldots$. . . . . . . . . . . . . . . . 80

5.4 .9 Monotonicity plus isotropy $\ldots \ldots \ldots \ldots$. . . . . . . 81

5.5 Scattering thresholds . . . . . . . . . . . . . . . . . 81

$5.5 .1 \quad$ 2-particle collisions . . . . . . . . . . . . . . . . . . 81

5.5 .2 Incoming 3 -velocities . . . . . . . . . . . . . . 83

5.6 Conclusions regarding threshold theorems $\ldots \ldots \ldots$. . . . . . 85

\begin{tabular}{lll}
\hline II & Thermodynamics of space-time & 87
\end{tabular}

\begin{tabular}{|lll}
6 & Thermodynamics of space-time - an overview & 89
\end{tabular}

\begin{tabular}{|lll}
\hline 7 & Clausius entropy for arbitrary bifurcate null surfaces & 93
\end{tabular}

7.1 Introduction . . . . . . . . . . . . . . . . . . . . . . 93

7.2 Flat Minkowski space-time $\ldots \ldots \ldots$. . . . . . . . . . . . . 96 


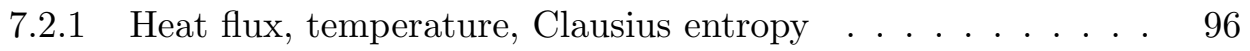

7.2 .2 Rindler wedges . . . . . . . . . . . . . . . . . . . . . 98

7.2 .3 Causal null cones . . . . . . . . . . . . . . . . . . . . . . 103

7.2 .4 Convex-base null conoids . . . . . . . . . . . . . . 106

7.2 .5 Causal diamonds . . . . . . . . . . . . . . . 107

$7.2 .6 \quad$ Generic null conoids . . . . . . . . . . . . . . . . . . . . 109

$7.2 .7 \quad$ Generic bifurcate null surfaces $\ldots \ldots$. . . . . . . . . . . . . 110

7.3 Compatibility with the Bekenstein bound . . . . . . . . . . . . . 111

$7.4 \quad$ Curved space-time $\ldots \ldots \ldots \ldots 112$

7.4 .1 Near the bifurcation 2-surface . . . . . . . . . . . . . . . 113

7.4 .2 General formula for curved-space Clausius entropy . . . . . . 115

$7.4 .3 \quad$ Generalized second law . . . . . . . . . . . . . . . . 115

7.5 Conclusions regarding Clausius entropy $\ldots \ldots \ldots$

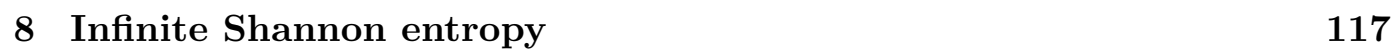

8.1 Introduction . . . . . . . . . . . . . . . . . . . . . 118

8.2 Some examples of infinite Shannon entropy . . . . . . . . . . . . . 119

8.3 Probability gap . . . . . . . . . . . . . . . . . . . . . . . . 120

8.4 Elementary bound leading to the Gibbs inequality . . . . . . . . . . 121

8.5 Partial counts, partial probabilities, partial Shannon entropies . . . . 122

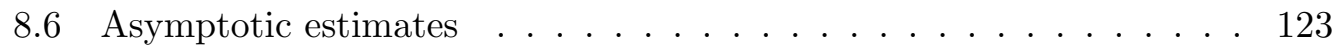

8.7 Entropy bounds from the Gibbs inequality . . . . . . . . . . . . . . . 124

8.8 Dirichlet series . . . . . . . . . . . . . . . . . . . . . . . 126

8.9 Discussion regarding infinite entropy $\ldots \ldots \ldots$. . . . . . . . . . . . 129

\begin{tabular}{lll}
\hline III Gravitational effects on quantum physics & 131
\end{tabular}

\begin{tabular}{|lll}
\hline 9 & Observing gravitational effects on quantum physics & 133
\end{tabular}

9.1 Introduction . . . . . . . . . . . . . . . . . . . . . 133

9.2 The experimental proposal $\ldots \ldots \ldots \ldots$

9.3 The Bose-Einstein condensate and acoustic metric . . . . . . . . . . 137

9.3 .1 Inertial and accelerated motion . . . . . . . . . . . . . . . 139

9.4 Bogoliubov transformations, the covariance matrix formalism and entanglement . . . . . . . . . . . . . . . . . . . 140

9.4 .1 Covariance matrix . . . . . . . . . . . . . . . . . . . . . 142

9.4 .2 Measurement of the entanglement - Negativity . . . . . . . . 143

$9.5 \quad$ Experimental setup . . . . . . . . . . . . . . . . . . . . 144 
9.6 Conclusions . . . . . . . . . . . . . . . . . . . . . . . . . . . . . . 149

\begin{tabular}{ll}
\hline 10 Summary and discussion & 151
\end{tabular}

\begin{tabular}{lll}
\hline IV Appendices & 155
\end{tabular}

\begin{tabular}{|ll}
\hline A Lorentz symmetry breaking & 157
\end{tabular}

A.1 Double special relativity and its applicability to our model . . . . . . 157

A.2 Relative locality . . . . . . . . . . . . . . . . . . . . . . 158

A.3 Some matrix identities . . . . . . . . . . . . . . . . . . 158

A.4 Consistency of dynamics and kinematics . . . . . . . . . . . . 159

\begin{tabular}{ll}
\hline B Energy conditions & 163
\end{tabular}

B.1 Average energy conditions . . . . . . . . . . . . . . . . . . 165

B.2 Some applications . . . . . . . . . . . . . . . . 167

\begin{tabular}{ll}
\hline C Generalised second law & 169
\end{tabular}

\begin{tabular}{|ll}
\hline D Jensen's inequality and Shannon entropy bounds & 171
\end{tabular}

D.1 Definition of Jensen's inequality . . . . . . . . . . . . . . . . . . . 171

D.1.1 Shannon entropy bounds . . . . . . . . . . . . . 171

\begin{tabular}{|lll}
\hline E & Bose-Einstein condensate and analogue space-time & 173
\end{tabular}

E.1 Effective space-time for a relativistic Bose-Einstein condensate . . . 173

E.2 Effective metric and accelerating BEC . . . . . . . . . . . . 175

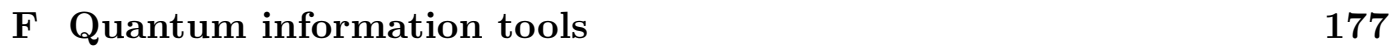

F.1 Covariance matrix formalism . . . . . . . . . . . . . . . . 177

F.2 Squeezed coherent states . . . . . . . . . . . . . . . . . . 178

F.3 Negativity as a measure of entanglement . . . . . . . . . . . . . . . . 181

G Publications not included in this thesis 183

G.1 Massive gravity from bimetric gravity . . . . . . . . . . . . . . . 183

G.2 Null Energy Condition violations in bimetric gravity . . . . . . . . . 184

G.3 Gordon and Kerr-Schild ansatze in massive and bimetric gravity . . 184

G.4 Massive gravity as a limit of bimetric gravity . . . . . . . . . . . . . 185

G.5 Inertial frames without the relativity principle: breaking Lorentz symmetry . . . . . . . . . . . . . . . . . . . . 185 
\begin{tabular}{lr}
\hline H Curriculum vitæ & 187
\end{tabular}

H.1 Education . . . . . . . . . . . . . . . . . . . . . . . . . . . . . . 187

H.2 Scholarship and prizes . . . . . . . . . . . . . . . . . . . . . 188

H.3 Other publications . . . . . . . . . . . . . . . . . . . . 188 


\section{List of Figures}

4.1 Transformations between inertial frames without relativity principle 32

4.2 First minimalist Lorentz-violating model . . . . . . . . . . . . . . . . 47

4.3 Second minimalist Lorentz-violating model $\ldots \ldots$. . . . . . . . . . 54

$5.1 \quad$ Kinematically accessible region for a Lorentz invariant particle of mass $m$, and a Lorentz invariant particle of mass $4 m$, decaying to two \begin{tabular}{|l}
\hline identical particles of mass $m . \ldots \ldots \ldots$ \\
\hline
\end{tabular}

$5.2 \quad$ Kinematically accessible region for a Lorentz invariant particle of mass $m$, and a Lorentz invariant particle of mass $4 m$, decaying to two identical particles with energy-momentum relation $E=\sqrt{E_{0}^{2}+E_{*}^{2} \tanh \left(p^{2} c^{2} / E_{*}^{2}\right)}$.

5.3 Kinematically accessible region for initial particles with a "latticelike" energy-momentum relation $E=(\hbar c / a)|\sin (p a / \hbar)|$, decaying to two identical particles with "tanh-like" energy-momentum relation $E=\sqrt{E_{0}^{2}+E_{*}^{2} \tanh \left(p^{2} c^{2} / E_{*}^{2}\right)} . \ldots \ldots \ldots \ldots$

5.4 Kinematically accessible region for a particle with a mass $m$ and a polynomial energy-momentum relation, decaying to two identical Lorentz invariant particles of mass $m$. . . . . . . . . . . 71

5.5 Kinematically accessible region for a particle of mass $m$ with a com-

plicated but monotonic energy-momentum relation, decaying to two
identical Lorentz invariant particles of mass $m$. . . . . . . . . . . 72

$5.6 \quad$ Plot of the energy as a function of $\epsilon$ for equation $(5.60)$ holding $E_{0}$ and $E_{*}$ fixed and for five distinct values of $p_{0} \ldots \ldots \ldots \ldots 79$

7.1 Rindler wedge: virtual hyperbolic timelike observer and 4-normal. . 99

7.2 Formal direction of the heat flux $₫ \mathrm{~d} Q . \ldots \ldots$. . . . . . . . . 100

7.3 Physical direction of the heat flux đQ, (and the entropy flux $\mathrm{d} S$ ), assuming the GSL holds. . . . . . . . . . . . . . . . 102 
7.4 Bifurcate double null cone based on a spherical bifurcation 2-surface. 104

7.5 Direction of physical entropy fluxes for the bifurcate double null cone based on a spherical bifurcation 2-surface. . . . . . . . . . . . 106

7.6 Causal diamond configuration for double null cones. . . . . . . . . . 108

7.7 Direction of the physical entropy fluxes, (assuming the GSL, which is implied by the NEC), for the causal diamond configuration. . . . . . 109

7.8 Bifurcation surface and the local Rindler horizon. . . . . . . . . . . . 113

9.1 Experimental proposal: Two BECs inside separate satellites are entangled while both are in the same circular LEO orbit; subsequently one undergoes a finite acceleration and a change of orbit. . . . . . . 136

9.2 Negativity $N$ versus difference in gravitational field potential between initial and final orbits $\delta \phi$, after the first change in velocity $\Delta v_{l}$ for different values of acceleration. . . . . . . . . . . . . . . . . . 145

9.3 Illustration of the CanX-4 and -5 nanosatellites. . . . . . . . . . . . . 146 


\section{List of Tables}

$5.1 \quad$ Behaviour at threshold for the 3-velocities and 3-momenta of outgoing final-state particles (compared to each other) under various assum-

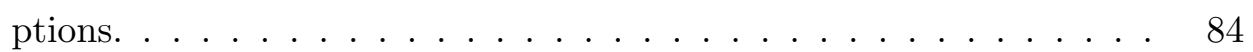

5.2 Behaviour at threshold for the 3-velocity and 3-momentum of the initial decaying particle (as compared to the final state decay product particles) under various assumptions. . . . . . . . . . . . . . 84

5.3 Behaviour at threshold for the 3-velocities and 3-momenta of the two initial state (incoming) particles (as compared to each other) under various assumptions. 


\section{Chapter 1}

\section{Introduction}

The historical development of science is characterised by several revolutions, to which one can refer as paradigm shift 1 . As is inherent in their definition the two last paradigm shifts, that had occurred at the beginning of the last century, had completely changed not only the interpretation of the physics world, but also the entire worldview within which it exists.

The theory of general relativity (GR) formulated by Albert Einstein in 1915, and the theory of quantum mechanics formulated during the first half of the twentieth century, could be considered the two greatest achievements of physics of the past century. Both describe in a comprehensive way the physical phenomena that fall under their domains, respectively cosmology and atomic and subatomic particles physics, and both do so to an astonishing degree of accuracy.

Yet they offer us strikingly different pictures of physical reality in which the description of "reality" given by the two theories seems to be quite in contradiction. Indeed, quantum mechanics and general relativity consist of two paradigms themselves. Quantum mechanics and its relativistic counterpart, quantum field theory, are formulated using quantities called dynamical fields on a fixed background, called Minkowski space-time, and they have an intrinsic probabilistic nature.

On the other hand the main and most interesting features of general relativity are the completely background independent formulation and the interpretation of gravity as a geometric property of space-time; GR is also a classical theory -

\footnotetext{
${ }^{1} \mathrm{~A}$ paradigm shift is the definition of scientific revolution given by the epistemologist and historian of science Thomas Kuhn in his book The Structure of Scientific Revolutions [190]. According to Kuhn, a paradigm shift occurs when the universally accepted theories, or paradigms, show anomalies that cannot be explained within paradigm itself. Kuhn identifies the paradigm not simply with the current theory, but the entire worldview in which it exists, and all of the implications which come with it.
} 
meaning it does not have any probabilistic/quantum behaviour.

For these reasons, these two theories turn out to be logically incompatible when applied to systems where they are both non-negligible, such as particle-physics processes at energy scales of the order of the Planck energy $E_{p} \sim 10^{19} \mathrm{GeV}$, therefore excluding the possibility of describing the very first moments of the Universe or the reality beyond the event horizon of a black hol $e^{2}$.

In principle one could keep this schizophrenic attitude and use the precise, geometric picture of general relativity while dealing with cosmological and (certain) astrophysical phenomena, while switching to the probabilistic theory of quantum mechanics when working with atomic and subatomic particles. Indeed - and rather unfortunately - the highest energy reached by our current technology, in the LHC particle accelerator, is of "only" $14 \mathrm{TeV}\left(14 \times 10^{3} \mathrm{GeV}\right)$, while the highest energy particles in nature ever registered, the trans-GZK cosmic ray 3 , can only reach energies of the order of $10^{11} \mathrm{GeV}$, still eight orders of magnitude less that the Planck scale, and consequently excluding any experimental access to energy regimes when both theories come into play. (Furthermore the trans-GZK particles are extremely rare, the total number of observed events between 1962 and 2008 ranging at a few dozen at the most, hence making the possibility of understanding any new physics rather difficult).

Nevertheless this picture is highly unsatisfactory from a conceptual point of view as the two theories consist of two paradigms that live in contradiction to one another. Certainly the successful quantum formulation and unification of three of the four fundamental interactions (electromagnetic, strong and weak, excluding gravity) led to the search of a theory of everything, of which the two pictures we are currently using can be considered approximations in their respective domains. This would be the provisional theory that we can use when faced with phenomena where the worlds of general relativity and quantum mechanics must unavoidably meet.

Quite remarkably, Einstein himself had already pointed out the necessity, if not of a quantum theory of gravity, at least of a theory that could take into account

\footnotetext{
${ }^{2}$ General relativity's configuration space is defined as diffeormophism invariant, i.e. invariant under active diffeormorphisms. However, whether the theory itself is invariant under this kind of diffeomorphism (especially "on shell") is still a matter of debate. However, when introducing matter degrees of freedom, the theory certainly loses such invariance, therefore allowing for the meaningful introduction of explicit energy scales such as the Planck energy.

${ }^{3}$ In astroparticle physics, an ultra-high-energy cosmic ray (UHECR) is a cosmic ray particle with a kinetic energy greater than $10^{18} \mathrm{eV}=10^{9} \mathrm{GeV}$, far beyond both its rest mass and energies typical of other cosmic ray particles. An extreme-energy cosmic ray (EECR) is an UHECR with energy exceeding $5 \times 10^{10} \mathrm{GeV}$, the so-called Greisen-Zatsepin-Kuzmin limit (GZK limit).
} 
quantum mechanics and his theory of gravitation at the same time. For instance in his 1916 article [104], published in the Preussische Akademie Sitzungsberichte, he wrote the following assertion:

- Nevertheless, due to the inneratomic movement of electrons, atoms would have to radiate not only electromagnetic but also gravitational energy, if only in tiny amounts. As this is hardly true in Nature, it appears that quantum theory would have to modify not only Maxwellian electrodynamics but also the new theory of gravitation.

The attempts in finding such an ultimate and unified theory have been ongoing, leading to several possible ways of addressing this quest. Already in the thirties, Bronstein, Rosenfeld and Dirac had produced some results, and more detailed work started to appear in the in the 1960s with the geometrodynamics (and quantum geometrodynamics) program, developed by Dirac, Bergmann, Arnowitt, Deser and Misner and others. For more details see for instance [42, 66, 177, 189], and Wheeler [299, 300].

Historically speaking, the great success of the quantisation of the electromagnetic field had led to the idea that gravity could be dealt with in the same way. However, in general relativity, with the metric playing the double role of fundamental dynamical field and space-time background, there is no definite separation between the kinematical part and dynamics, and already at the classical level substantial efforts are required to formulate physical questions. When moving to the quantum theory, the presence of the uncertainty principle would further complicate the matter, since after evolving an initial state one would not obtain a unique space-time.

In spite of all these difficulties, over more than fifty years several approaches have been developed that have used and modified some of the varied quantisation techniques originally developed for quantum field theories. For instance we can observe that to a good approximation the canonical quantisation approach was pursued by relativists while the covariant one by particle physicists, adopting completely different attitudes to facing the technical problems. Here we summarise some characteristics of the most relevant approaches.

- Geometrodynamics follows the canonical quantisation path, with the GR Hamiltonian generating a time evolution. In this case the emphasis is on preserving the geometrical character of GR. The canonical variable is the 3-metric on a spatial slice, and in this formulation GR can be interpreted as the dynamical theory of 3-geometries (hence the name). Unfortunately the quantum 
theory developed using this formalism encountered some theoretical difficulties associated with an infinite number of degrees of freedom, and created a split between general relativity and the theory of elementary particles. Nevertheless this approach is pivotal for the consequent development of the whole field, [299, 300].

- Loop quantum gravity (LQG) follows the same line and makes use of the canonical quantisation method; in this case though the canonical variables are the spin-connections, required to parallel propagate spinors, and GR can be regarded as dynamical theory of connections [49. The main advantage of such approach is that the phase-space of the theory is the same as for a gauge theory, so that techniques that had been implemented in the quantisation of the gauge theories can now be transferred to general relativity. In the case of $3+1$-dim the quantum states are taken to be suitable functions of Wilson loops, or graphs, on the 3-manifold, from which the theory takes its name [240, 242, 243].

- Spin foam models had been initially developed as attempt to provide LQG with an appropriate path integral formalism. Following the lessons of quantum field theory, the transition amplitudes are calculated as a sum over paths interpolating between two states. However the spin foam framework had already arisen in the context of many of the known topological quantum field theories, like Ponzano-Regge model of gravity in three dimensions, or $B F$ theory [50, 225, 226, 231].

- Causal dynamical triangulation is a modification of quantum Regge calculus where smooth space-time is discretised with a process called triangulation. In other words it is approximated by a network of triangulation nodes (or simplices), where space is locally flat but globally curved, as with the individual faces and the overall surface of a geodesic dome. The crucial development, which makes this a relatively successful theory, is that the network of simplices is constrained to evolve in a way that preserves causality. This allows a path integral to be calculated non-perturbatively, by summation of all possible (allowed) configurations of the simplices, and correspondingly, of all possible spatial geometries [15, 16, 202].

- Hořava-Lifshitz gravity [156] is an approach to quantum gravity that breaks Lorentz invariance at ultra-high (presumably trans-Planckian) energies, to regularise the infinities of the theory. It is defined as a traditional (point-particle) 
quantum field theory, therefore it is not a string theory, nor loop quantum gravity. The novelty of this approach is the use of concepts from condensed matter physics such as quantum critical phenomena, to give a measure of the degree of anisotropy between space and time.

- String theory can be included within those approaches that use the covariant formalism for the quantisation process; however its origin is slightly different than that of the models listed so far, in that it was first introduced to study the strong interactions. In particular point-particles are replaced by one-dimensional extended objects - strings - and particle-like states are associated with various modes of excitation of the string. In this model gravity is somehow automatically incorporated since in addition to spin-1 modes, associated with gauge theories, it included a spin- 2 massless excitation, the graviton [60, 229, 230].

All these approaches have produced a huge quantity of results and insights; unfortunately, given the lack of any experimental evidence that could provide us with a conclusive and definite theory, we may be forced to take other routes.

As we have seen, the motivation behind several of the models presented above lies in the successful quantisation of electromagnetism starting from the classical theory. However, it may well be that electromagnetism is the only case in nature for which the quantisation leads to the actual quantum degrees of freedom. Even in quantum chromodynamics the fundamental particles are the quarks, while the emergent degrees of freedom are hadrons and mesons.

For this reason, it would be worth trying to address the problem by searching for some plausible microscopic theory whose asymptotic, classical limit is the Einstein theory of gravitation. Since there is no gravity correspondent for the Stern-Gerlach experiment or double slit experiment, one may want to use, as a starting point, some of the theoretical results upon which hard core theories, as those listed above, agree. For instance, both covariant and canonical theories agree on the fact that space-time becomes somehow fuzzy or discrete at the Planck scale, possibly leading to the breaking of the Lorentz symmetry.

In this thesis work we shall focus on some of those physics phenomena that probe the quantum/gravity regime and which could unveil the next theoretical or experimental steps in this quest. We do not directly expect to find the ultimate theory that will unify all the four interactions in physics, neither a quantum theory of gravitation. Instead we would like to follow the lesson learned from the derivation 
of the thermodynamic nature of black holes. In this case, three fundamental parts of physics had been brought to work together: general relativity, quantum theory and statistical mechanics.

This thesis is divided in three main parts. In the first part, we shall introduce the subject of physics without Lorentz invariance. Lorentz symmetry is well established for the energy regimes that we are able to test; however there have been repeated theoretical suggestions that it may not be an exact symmetry at all energies. For instance four dimensional Lorentz violation has been investigated in different quantum gravity models - string theory, loop quantum gravity, warp brane worlds, Hořava-Lifshitz gravity, etc. - leading to different theoretical frameworks within which Lorentz symmetry might be modified or even broken. In general, the notion is that there is strong Lorentz violation at the Planck scale that decreases at lower energies. For this reason, it is worth exploring possible theories that contemplate the possibility of Lorentz violation.

In particular in chapter 4 we shall investigate the case of Lorentz symmetry breaking when one renounces the relativity principle. In 1910 von Ignatowsky gave a mathematical description of special relativity that established a very tight connection between the group structure implied by the relativity principle and the rules for the transformation of space-time coordinates [285-288]; a connection that, combined with the basic and fundamental physical assumptions of locality, linearity, and isotropy, leads almost uniquely to either the Lorentz transformations of special relativity — or to Galileo's transformations of classical Newtonian mechanics without making any a priori appeal to the constancy of the speed of light.

Therefore, if one wishes to abandon Lorentz symmetry within the context of the class of local physical theories, then it seems likely that one will have to either modify or even discard the relativity principle. Our purpose is to redefine the notion of space-time transformations between inertial frames in the absence of the relativity principle; we shall argue that the choice of inertial frame implies at least linearity for the coordinate transformations - to map straight lines into straight lines.

Under these circumstance we shall demonstrate that the set of coordinate transformations now form a groupoid/pseudo-group, i.e. closed only under partial-products, and it is exactly this technical difference that permits one to evade the von Ignatowsky argument. Even in the absence of a relativity principle we can nevertheless deduce clear and compelling rules for the transformation of space and time and rules for the composition of 3 -velocities.

The next step in our derivation regards the definition of the transformations for energy and momentum, necessary concepts if one wants to go from the kinematic to 
dynamic regime and set up some models within which to test the theoretical results. In section 4.3 we shall show that energy-momentum transformations are in general affine - linear plus an inhomogeneous offset - but may be chosen to be linear.

As an application of the results we have found for space-time and energy-momentum transformations, we shall illustrate two physically compelling models implementing "minimalist" violations of Lorentz invariance. In the first one all Lorentz violations are confined to carefully delineated particle physics sub-sectors; that is the space-time coordinates transform with Lorentz transformations, while the transformations for energy and momentum are of the kind mentioned above. The second minimalist Lorentz-violating model, space-time transformations depend on one free function of absolute velocity, but otherwise preserve as much as possible of standard Lorentz invariant physics.

In chapter 5 we shall continue along the line of investigating Lorentz symmetry breaking by considering thresholds for decay and scattering processes. For these kinds of processes the analysis becomes considerably more complicated and extremely subtle, with many new and naively unexpected effects. Based only on the assumption of the existence of some dispersion-relation between energy and momentum $E(p)$, we shall develop several threshold theorems without assuming any isotropy or monotocity for the relation $E(p)$. Indeed several physically interesting situations exist where such a level of generality is called for, some examples are lattice QFT regularisations, leading to energy-momentum relations that are similar to energy-momentum relations for quasi-particles propagating through atomic lattices. In the various parts of the chapter we shall show that even in this most general of settings, at threshold all final state particles move with the same 3-velocity, while initial state particles must have 3-velocities parallel/anti-parallel to the final state particles. Quite remarkably, we shall see that, contrary to the behaviour of the 3velocities, the various 3 -momenta can behave in a complicated and counter-intuitive manner.

In the second part we shall introduce the subject of thermodynamics of spacetime. With this definition we mean the possible interpretation of general relativity as a mean field theory that arises from some underlying microscopic degrees of freedom. This point of view on GR, known as induced gravity, was first formulated by Andrei Sakharov in 1967, and interprets space-time geometry as an emergent property similar to hydrodynamics emerging from molecular physics [247, 277]. As we shall see in chapter 6 , the thermodynamic interpretation is brought about by considering the special case of black hole physics and the strict analogy between 
the four laws of thermodynamics and those of black hole mechanics [58, 62, 142]. Another result that seems to reinforce this interpretation is the derivation of the Einstein field equation as an equation of state by Ted Jacobson in 1995 [157]. In this case the author begins by considering the area law for a Rindler horizon that is the proportionality of the entropy of the horizon to its area rather than its volume - and some heat flux across it that perturbs the underlying metric. Using this assumption, the Clausius definition of the entropy đQ $=T d S$, and the fact that the flux at the bifurcation point ${ }^{4}$ needs to be zero - implying that the null geodetics need to focus in that point - Jacobson obtained Einstein equations from thermodynamic relations.

In chapter 7 we shall consider part of Jacobson's derivation particularly focusing on the definition of the thermodynamic system, and generalising it to a generic bifurcate null surface. Given this general construction, that utilises virtual accelerating observers, the question arises concerning the ontological nature of the entropy, i.e. if entropy is objectively "real" or if it is in some sense subjective and observer-dependent. This matter is deep-rooted in the fact that there exist several definitions of entropy such as Clausius entropy, thermodynamic entropy, statistical entropy (Shannon or von Neumann entropy), or Bekenstein entropy, and their equivalence is less than clear. In this chapter we shall assign a notion of Clausius entropy to arbitrary bifurcate null surfaces - effectively defining a "virtual Clausius entropy" for arbitrary "virtual (local) causal horizons". As an application, we see that we can implement a version of the generalised second law [63] for this virtual Clausius entropy. As the reader will see, we have taken some effort in being careful and explicit in developing our framework, given the subtle concepts utilised in the derivation.

Entropy seems to play a critically important role in the study of the thermodynamic nature of space-time; however several definitions coexist in the literature, and the extent to which they are equivalent is far from definite. For this reason we think it is relevant to start by studying the mathematical characteristics of the entropy to which we shall dedicate chapter 8 . For the time being we shall consider single-channel Shannon entropy in the information theoretic sense, not entropy in a stochastic field theory or quantum field theory (QFT) defined over some configuration space, on the grounds that this simple problem is a necessary precursor to understanding infinite entropy in a field theoretic context.

In particular what we shall demonstrate is that even though a probability dis-

\footnotetext{
${ }^{4}$ The bifurcation point, that we shall later indicate with $\mathcal{P}$, is a point on a two dimensional space-like surface upon which one attaches the null surface.
} 
tribution is properly normalisable, its associated Shannon (or von Neumann) entropy can easily be infinite. Roughly speaking, this happens when arbitrarily small amounts of probability are dispersed into an infinite number of states. In particular, we shall see that large entropies cannot be localised in state space; large entropies can only be supported on an exponentially large number of states.

In the third part of this thesis we shall introduce an experimental proposal to test the effects of acceleration and gravity on quantum physics. Following the line of what we have said above, one would like to find alternative ways to understand the quantum nature of space time. In this case we would like to reverse the usual logic, i.e. studying quantum effects at large scales. Indeed this seems to be experimentally achievable in the near future as cutting-edge quantum experiments are reaching relativistic regimes, where the effects of gravity and motion on quantum properties can be experimentally tested - see for instance the experiments proposed in [201, 213, 237, 251, 306] or the successful teleportation protocol that was performed across $144 \mathrm{~km}$ by the group led by A. Zeilinger [272].

In this respect, there are some previous theoretical works that had already addressed these fundamental questions by showing that gravity, motion and space-time dynamics can create and degrade entanglement [14], and that acceleration produces observable effects on quantum teleportation [125]. However, current experimental space-based designs are yet to consider these findings. In this chapter we propose a space-based experiment to test the effects of gravity and motion on quantum entanglement. The novelty of the experiment we shall talk about in chapter 9, with respect to the other proposals that have been developed in the framework of quantum mechanics, is the use of the quantum field theory, therefore incorporating Lorentz invariance and causality.

In this chapter we shall demonstrate that the gravitational field of the earth and accelerated motion can induce experimentally observable effects on quantum entanglement - that constitutes the basic resource for quantum information and quantum communication tasks. In principle, these results could shed some light on fundamental questions about the overlap of quantum theory and relativity and, at the same time, they may enable experimentalists interested to implement them into spacebase technology, to correct negative gravitational effects on quantum technologies on satellites.

In more detail, a recent result has shown that the entanglement between field modes of localised systems, such as cavities, is sensitive to changes in acceleration [74. Via the equivalence principle, this means that entanglement may be affected 
by changes in gravitational field strengths. In order to demonstrate this experimentally we shall consider the entanglement between the phononic excitations of two Bose-Einstein condensates (BECs), each one of them prepared in a separate satellite. These excitations obey, under certain circumstances, a massless Klein-Gordon equation with a very slow speed of propagation [227]. As we shall explain in more detail, a low propagation speed is the key element to enable the observation of the effect we describe within realistic experimental regimes.

The next step will be to entangle the two BEC modes, one in each BEC, while the BECs are into two nano satellite $5^{5}$ that move close to each other along the same circular earth orbit. One of the satellites will then undergo a non-uniform motion to change to an orbit subject to a different gravitational field potential. The interesting result is that the entanglement degradation between the BEC phononic modes is a periodic function of the change in gravitational field potential in the orbit. Moreover this effect is significant already for typical parameters involved in microsatellite manoeuvres.

As usual, in the final part of the thesis we shall draw conclusions for all the topics we have discussed in the previous chapters, as well as outline possible future research paths one can undertake and the expected results. In appendices $\mathrm{A}$ to F the reader can find a few mathematical tools that have been used in chapter 4 . chapter 7, chapter 8 , and 9, along with some in-depth sections about notions that have been only briefly mentioned. Appendix $\mathrm{G}$ lists the publications and conference proceedings articles that have not been included in this thesis work.

\section{Distribution of credits}

The scientific results included in this thesis work are the product of three years of $\mathrm{PhD}$ research I have carried out with my supervisor and other colleagues in the School of Mathematics, Statistics and Operations Research at the Victoria University of Wellington, New Zealand, and while visiting the relativistic quantum information group in the School of Mathematical Sciences at the University of Nottingham, UK. The results of chapter 4 and chapter 5 are the outcome of a joint effort of myself, my supervisor, Prof. Matt Visser and my colleague and friend Kyle Tate. Each of us gave a substantial contribution in terms of discussions, exchanges of ideas and careful calculations. The results have been published in [45] and [46].

The results of chapter 7 and 8 have been obtained by me and my supervisor,

\footnotetext{
${ }^{5} \mathrm{~A}$ nanosatellite is an artificial satellite whose wet mass spans from 1 to 10 kilograms, with a size of about 10 centimetres per side.
} 
Prof. Visser. As for the results in chapters 4 and 5 , both of us had give a substantial contribution in terms of discussions, exchanges of ideas and careful calculations. These results have been published in [48] and [47].

The experimental proposal that I shall present in chapter 9 is the result of a collaboration with the relativistic quantum information group at the school of mathematical sciences of University of Nottingham, the UK. My main contribution can be found in section 9.3 , while the rest has been a collaborative effort developed by Dr. David Edward Bruschi, Dr. Carlos Sabín, Dr. Angela White, Dr. Daniel Oi, Prof. Ivette Fuentes and partially by myself. A publication based on this project can be found in [75]. 


\section{Chapter 2}

\section{Notation}

Throughout this thesis work we shall use the following notation. For the Minkowski metric we use the convention:

$$
\eta_{a b}=\left[\begin{array}{cccc}
-1 & & & \\
& 1 & & \\
& & 1 & \\
& & & 1
\end{array}\right] .
$$

We use $a, b=(0, \ldots, n)$ with $n=3$ for a $3+1$ space (such as in this case); we shall use the letter $i, j$ for the spatial components of vectors matrices $i, j=(1, \ldots, n)$. For Lorentzian metric we shall consider the signature $(-,+,+,+)$. In general we shall utilise natural units $c=\hbar=G=k_{B}=1$, unless specified in the chapter. 


\section{Part I}

\section{Physics without Lorentz invariance}





\section{Chapter 3}

\section{Lorentz symmetry breaking overview}

In this part of the thesis we shall present the topic of Lorentz symmetry breaking. In particular we shall focus onto two aspects: breaking Lorentz symmetry by renouncing the relativity principle, and analysis of thresholds in both scattering and decay processes in the case Lorentz invariance is violated.

One may wonder why we would want to abandon such an experimentally welltested symmetry as Lorentz invariance. After all special relativity has been one of the most successful theories of the last century and it can be considered a cornerstone of modern physics.

Certainly, there exist several reasons behind this argument, based on a variety of considerations. There are purely theoretical considerations, mainly related to a theory of quantum gravity (see below), and there are more pragmatic ones such as the need for a phenomenological framework within which to formulate empirical tests of Lorentz invariance, and formulate appropriate questions.

Over the last decade significant progress along these lines has been made. See for instance work by Coleman and Glashow [88, 89], Amelino-Camelia, Ellis, Mavromatos, Nanoplous, and Sarkar [22, Gambini and Pullin [126], Kifune [174, Aloisi, Blasi, Ghia, and Grillo [12], Amelino-Camelia and Piran [27, plus that by Jacobson, Liberati, and Mattingly [158-165, 198, 211], and especially the Living Review by Mattingly [210].

The net result of all these efforts is the considerable quantity of observational bounds, some of them very stringent observational bounds, constraining the possibility of Lorentz symmetry breaking - although it should perhaps be noted that these analyses are typically performed in the preferred (aether) frame. 
However, related more to the purpose of this thesis work is the theoretical point of view. Indeed there have been numerous and long-standing suggestions that Lorentz invariance may not be an exact symmetry at all energies; in other words several theories of quantum gravity have predicted an eventual violation of Lorentz invariance at the Planck scale.

This possibility has been investigated, for instance, in the string-inspired theoretical framework developed by Kostelecky and collaborators for characterizing possible violations of Lorentz invariance [59, 91, 180 186]. More recently, the Horava gravity framework [156] naturally includes Lorentz violation [261, 262, 279 281, 297]. The "analogue space-time" programme also very naturally leads to models where Lorentz invariance is violated at one level or another [55, 56, 276, 283]. There is also the flat-space non-gravity framework developed by Anselmi [28 40], where Lorentz invariance breaking is used to partially regulate QFT ultraviolet divergences. Further afield, Nielsen and collaborators have studied the renormalization group flow of Lorentz symmetry violating operators in generic QFTs, demonstrating that Lorentz invariance is often an infrared fixed point of a generic Lorentz violating QFT [80, 214,217].

For the above-mentioned reasons, it should be clear to the reader why it is interesting to study possible Lorentz violations. In particular, we know that, if Lorentz symmetry is violated by quantum gravity, one would expect to set the Planck energy of $E_{p} \approx 10^{19} \mathrm{GeV}$ to be the natural scale at which strong violation effects become manifest. Unfortunately, this range of energies resides far beyond the capability of our current experiments (LHC can reach energies of "only " $14 \mathrm{TeV}$ ), and even beyond the highest known energy particles, the trans-GZK cosmic rays of $10^{11} \mathrm{GeV}$.

However, one would expect to see some remnant of the interpolation between the Lorentz-violating Planck scale, and the low-energy, Lorentz-invariant world we live in - with a small amount of Lorentz violation present at all energies. Furthermore, great advances in technology and observational techniques have effectively improved the precision of the experimental test to the point where small residual effects of Planck scale Lorentz violation could be detected.

For this reason, in chapter 4 , we shall explore the modification of the transformations of time and space between inertial frames when Lorentz symmetry breaking is induced by renouncing the relativity principle. Indeed, given this level of interest in the topic, we have feel that it is interesting, useful, and timely to perform a careful analysis of the general and very basic notion of inertial frames in the absence of Lorentz invariance. We shall focus particularly on "preferred frame" (aether) ver- 
sions of Lorentz symmetry breaking - that is, we shall study inertial frames in the absence of the relativity principle, but while retaining usual notions of local physics. In chapter 5 we shall investigate what happens to threshold theorems for scattering and decay processes when Lorentz symmetry is no longer fulfilled.

In appendix $\mathrm{A}$ the reader can find a few comments regarding the applicability of our results to different Lorentz symmetry breaking schemes (e.g double special relativity). Useful mathematical identities for the results in chapter 4 are also presented. 


\section{Chapter 4}

\section{Inertial frames without the relativity principle}

\subsection{Introduction}

In this chapter we shall illustrate what is the most suitable method of minimally breaking Lorentz symmetry, should one want to preserve some basic concepts such as that of inertial frame and local physics. At the same time we shall explore how this would affect the well-known results of special relativity, and how fundamental physics phenomena are consequently changed. Even more crucial, we shall investigate if some meaningful physics can still be carried out.

Before starting to tackle this matter, we would like to illustrate why we want to minimally break Lorentz symmetry. The reason lies in a lesson learned from experimental physics, that is one should "only adjust one parameter at a time". The theorist's equivalent is that one should "only adjust one theoretical assumption at a time". Controlled restraint in relaxing one's input assumptions is essential if one is to develop a pragmatically useful framework that is sufficiently well-defined to make definite statements that can in principle be confronted with empirical reality.

For this reason, our attempts started by singling out the pivotal components of special relativity. In order to do that we focused on the von Ignatowsky mathematical description of special relativity (1910), that established a very tight connection between the group structure implied by the relativity principle and the rules for the transformation of space-time coordinates [285,288].

In particular, von Ignatowsky's formulation attests that, under suitable hypotheses — such as locality, linearity and isotropy - the relativity principle almost uniquely leads to either the Lorentz transformations of special relativity, or Galileo's 
transformations of classical Newtonian mechanics. Moreover this result makes no a priori appeal to the constancy of the speed of light. Over the last century this same result has been repeatedly rediscovered, expanded upon, and re-analyzed, with significant pedagogical efforts being expended; see for instance [67, 68, 114, 120, 121, 132, 133, 166, 192, 194, 196, 199, 204, 223, 238, 255, 265, 267, 269, 301.

The relevance of von Ignatowsky's analysis for our current purposes comes from reversing the logic: If for whatever reason one wishes to speculate about a possible breakdown of Lorentz invariance at ultra-high energies, then as long as one continues to work within the framework of classical local physics one is almost certain to be forced to abandon, or at the very least grossly modify, the relativity principle and in particular one will in general be forced to abandon the group structure for the set of transformations that connect space and time in different inertial frames.

The specific question we shall answer is the following: Within the framework of local physics, what happens to inertial frames, and the transformations between inertial frames, if you do not have the relativity principle? We shall see that quite a lot can still be said. Under suitable hypotheses, it is possible to argue that the space and time transformation rules between inertial frames should at least be linear. (We shall subsequently have a few words to say about situations where these transformations might not be linear.) For linear transformations between inertial frames, rather general formulae for the transformation of 3-velocities, and in particular the composition of 3 -velocities, can then be derived.

In this case, the set of transformations between inertial frames forms a groupoid/ pseudogroup. (In this particular sub-branch of mathematics the mathematical terminology is not $100 \%$ settled.) It is exactly the distinction between a groupoid/pseudogroup and a group that will allow us to evade von Ignatowsky's argument.

The subsequent step is explore the possibility of going beyond the most basic of kinematics, that is, develop some notion of dynamics in the specific framework of local theories without the relativity principle. To do so requires one to develop some notion of energy and momentum - thereby implying the ability to construct some Lagrangian or Hamiltonian mechanics. Specifically, to understand the dynamics in Lorentz violating theories it is necessary to understand how energy and momentum transform, and to understand how the Lagrangian and Hamiltonian transform. In the context we are considering - local theories with linear transformations between inertial frames - the generic situation is that energy and momentum transform in an affine manner (that is, linear plus an inhomogeneous offset term).

We shall show that it is possible, but not always desirable, to choose conventions and parameters in such a way as to force the offset to be zero - in which case energy 
and momentum transform in a homogeneous linear manner. In fact, if this is done, then with our conventions the 4-component vector $P=\left(E,-\boldsymbol{p}^{T}\right)$, the 4-momentum, is a row vector. $P$ is an element of the vector space dual to the 4 -component vector $X=\left(t, \mathbf{x}^{T}\right)^{T}$, the 4-position, which is a column vector. (We shall see that the offset term in the affine transformation is needed if one wishes to recover the usual naive form of Newtonian mechanics in a suitable limit, but that there is a somewhat nonstandard formulation of Newtonian mechanics in which energy-momentum can be made to transform linearly.)

Finally, since our main purpose is to develop some tests that can give us a hint of where and what to look in an experimental setting, we shall focus on two particularly elegant and compelling models implementing a minimalist violation of Lorentz invariance. The first minimalist Lorentz-violating model confines all Lorentz violating physics to some suitable sub-sector of the particle physics spectrum (most typically taken to be the neutrino sector). The second minimalist Lorentz-violating model preserves as much as possible of standard Lorentz invariant physics, but the transformations additionally depend on one extra function, an arbitrary free function of absolute velocity. Consequently, when studying possible violations of Lorentz invariance, these two models in many ways serve as examples of "leastdamage" violations of Lorentz invariance. Indeed, the considerations we have drawn up will be essential to almost any form of violation of Lorentz invariance that respects locality and encodes "preferred frame" (aether frame) effects.

\subsection{General transformations between inertial frames}

As is well known, an essential feature one wants to preserve when formulating a physics theory is the possibility of comparing its predictions with some experimental results. In order to do that one needs to be able to compare results between different observers, establishing how clocks and rulers change when going from one reference frame to another.

For this reason, the first step is to define the kind of reference frame we want to consider and how space and time are related among them.

\subsubsection{Definition of an inertial frame}

Since we would like to modify only a few features at a time, we shall begin by considering inertial frames; in this way we shall avoid introducing any possible exotic effect from the very beginning. Generally speaking, everyone would agree on this characterization of inertial frames: 
1. All inertial frames are in a state of constant, rectilinear motion with respect to one another; they are not accelerating, in the sense of proper acceleration that would be detected by an accelerometer.

2. In an inertial reference frame, the laws of mechanics take their simplest form.

3. In an inertial frame, Newton's first law (the law of inertia) is satisfied: Any free motion has a constant magnitude and direction, implying a linear relationship between the space and time coordinates assigned to any free particle.

In order to obtain the usually accepted formulation of physics, one would introduce the additional and extremely stringent condition of the relativity principle that states:

Laws of physics take the same form in all inertial frames.

But, as we have argued above, and as we shall show in the following, one can renounce the relativity principle and still be able to deduce some meaningful results. And that is the topic we shall now explore.

\subsubsection{Argument for linearity}

Even in the absence of the relativity principle, we shall still want the transformations between reference frames to be linear. As we shall see, such requirement fulfils the definition of rectilinear motion in inertial frames (necessary to satisfy Newton's first law).

In particular, by definition a freely moving particle, in an inertial frame, is not accelerating

$$
\frac{d^{2} \mathbf{x}}{d t^{2}}=0 ; \quad \mathbf{x}(t)=\mathbf{x}_{0}+\boldsymbol{v}_{0} t
$$

Since we want to preserve such kind of motion in any other inertial frame, the particle is again by definition not accelerating

$$
\frac{d^{2} \overline{\mathbf{x}}}{d \bar{t}^{2}}=0 ; \quad \overline{\mathbf{x}}(\bar{t})=\overline{\mathbf{x}}_{0}+\overline{\boldsymbol{v}}_{0} \bar{t} .
$$

Whatever the transformation is between the two sets of time and space coordinates $\{t, \mathbf{x}\}$ and $\{\bar{t}, \overline{\mathbf{x}}\}$, the transformation has to map straight lines into straight lines - which forces the transformation to be, at the very worst, projective [114, 238]. By additionally requiring that events in a bounded region (of space-time) map into a bounded region (of space-time) this is actually enough to force the transformations to be linear [114, 238]. 


\section{Some technical considerations}

If one is only interested in implementing Newton's first law, then one could get away with using an arbitrary abstract vector space defined over an arbitrary abstract number field. Newton's first law would still require straight lines to map into straight lines, and so would naturally lead to projective transformations. By considering bounded regions of a normed vector space it becomes natural to restrict attention to linear transformations between inertial frames. But as soon as one wishes to implement Newton's second law, one needs to be able to discuss non-zero variable acceleration, which requires some notion of differentiability, which naturally leads one to consider a vector space defined over the real numbers.

For our purposes we write the 4-position as a column vector

$$
X=\left(\begin{array}{c}
t \\
\mathbf{x}
\end{array}\right) \text {, }
$$

and we want it to transform in the following guise

$$
X \rightarrow \bar{X}=M X .
$$

We adopt the conventions where both 3 -vectors $\mathbf{x}$ and 4 -vectors $X$ are column vectors to minimize the number of special case notational fiddles we have to adopt later on in the discussion.

\section{Some considerations on inertial frames and linearity}

On a technical note, we want to stress that the primary physics input is the observation that inertial frames exist, and from extremely basic notions of kinematics this is enough to argue for linearity. If one additionally wants to develop some notion of Lagrangian/Hamiltonian dynamics - as we shall see later on - then the observation that free inertial particles exist, coupled with Noether's theorem, can be used to argue for the homogeneity of space and time. Some authors prefer to start from homogeneity, and thereby deduce linearity. There are minor technical issues, but for all practical purposes space-time homogeneity implies and is implied by linearity of the transformations between inertial frames.

We emphasise how basic and fundamental the argument for linearity is - if the transformation law between inertial frames is not linear, then it is really the whole notion of inertial frame that is being undermined. 


\subsubsection{General representation of inertial transformations}

Now that we have reviewed that the concept of inertial frame and linearity are strongly intertwined, we shall briefly recall what is their implication with respect to the transformations between different reference frames in some well-known (and less-known) physics theories.

Newtonian physics: If one considers the special case of Newtonian physics (Galilean relativity) we have that space and time transform as

$$
M=\left[\begin{array}{c|c}
1 & \mathbf{0}^{T} \\
\hline-\boldsymbol{v} & I
\end{array}\right] .
$$

Einstein physics: In the case of special relativity we recall the familiar form for the transformation of the 4-positions

$$
M=\left[\begin{array}{c|c}
\gamma & -\gamma \boldsymbol{v}^{T} / c^{2} \\
\hline-\gamma \boldsymbol{v} & \gamma \mathbf{n} \mathbf{n}^{T}+\left[I-\mathbf{n n}^{T}\right]
\end{array}\right],
$$

with $\boldsymbol{v}=v \mathbf{n}$ and $\gamma=\left(1-v^{2} / c^{2}\right)^{-1 / 2}$. One can also write this as

$$
M=\left[\begin{array}{c|c}
\gamma & -\gamma \boldsymbol{v}^{T} / c^{2} \\
\hline-\gamma \boldsymbol{v} & \gamma \mathbf{n} \otimes \mathbf{n}+[I-\mathbf{n} \otimes \mathbf{n}]
\end{array}\right] .
$$

As is well known, this can be reduced to a product of a spatial rotation and a boost in an arbitrary direction.

Carroll kinematics: This kind of kinematics is also knowns as "Alice in wonderland kinematics". It is a rarely encountered and somewhat unphysical limit of the Lorentz group where one takes $c \rightarrow 0$ and $v \rightarrow 0$ while keeping the "slowness" $u=v / c^{2}$ fixed. The resulting transformations

$$
M=\left[\begin{array}{c|c}
1 & -\boldsymbol{u}^{T} \\
\hline \mathbf{0} & I
\end{array}\right]
$$

correspond to [95, 103, 134, 155, 195]:

$$
t \rightarrow \bar{t}=t-\boldsymbol{u} \cdot \mathbf{x} ; \quad \mathbf{x} \rightarrow \overline{\mathbf{x}}=\mathbf{x}
$$

We will have very little to say concerning this particular option. 


\section{A more general scenario}

In the case where we do not have any relativity principle, we shall consider the transformation $M$ to be some matrix which we can, without loss of generality, write in the form

$$
M=\left[\begin{array}{c|c}
\gamma & -\boldsymbol{u}^{T} \\
\hline-\boldsymbol{w} & \Sigma
\end{array}\right]
$$

We shall also introduce the inverse transformation since we want to define a theory where one can transform back from one reference frame to the other:

$$
M^{-1}=\left[\begin{array}{c|c}
\left(\gamma-\boldsymbol{u}^{T} \Sigma^{-1} \boldsymbol{w}\right)^{-1} & \left(\boldsymbol{u}^{T} / \gamma\right)\left(\Sigma-\boldsymbol{w} \boldsymbol{u}^{T} / \gamma\right)^{-1} \\
\hline\left(\gamma-\boldsymbol{u}^{T} \Sigma^{-1} \boldsymbol{w}\right)^{-1} \Sigma^{-1} \boldsymbol{w} & \left(\Sigma-\boldsymbol{w} \boldsymbol{u}^{T} / \gamma\right)^{-1}
\end{array}\right]
$$

The matrix $M$ is the most generic kind of transformation that preserves linearity and specifically, we are not at this stage assuming any notion of isotropy. Note that the object $\boldsymbol{w} \boldsymbol{u}^{T}=\boldsymbol{w} \otimes \boldsymbol{u}$ is a $3 \times 3$ matrix, while $\boldsymbol{u}^{T} \boldsymbol{w}=\boldsymbol{u} \cdot \boldsymbol{w}$ is a scalar.

There are several possible forms that one can choose to describe the matrix $M$. For instance, by replacing $\boldsymbol{u} \rightarrow \gamma \boldsymbol{u}$, we could write this in the completely equivalent form

$$
M=\left[\begin{array}{c|c}
\gamma & -\gamma \boldsymbol{u}^{T} \\
\hline-\boldsymbol{w} & \Sigma
\end{array}\right]
$$

and its inverse

$$
M^{-1}=\left[\begin{array}{c|c}
\gamma^{-1}\left(1-\boldsymbol{u}^{T} \Sigma^{-1} \boldsymbol{w}\right)^{-1} & \boldsymbol{u}^{T}\left(\Sigma-\boldsymbol{w} \boldsymbol{u}^{T}\right)^{-1} \\
\hline \gamma^{-1}\left(1-\boldsymbol{u}^{T} \Sigma^{-1} \boldsymbol{w}\right)^{-1} \Sigma^{-1} \boldsymbol{w} & \left(\Sigma-\boldsymbol{w} \boldsymbol{u}^{T}\right)^{-1}
\end{array}\right] .
$$

Alternatively, by now replacing $\boldsymbol{w} \rightarrow \Sigma \boldsymbol{w}$,

$$
\begin{gathered}
M=\left[\begin{array}{c|c}
\gamma & -\gamma \boldsymbol{u}^{T} \\
\hline-\Sigma \boldsymbol{w} & \Sigma
\end{array}\right] ; \\
M^{-1}=\left[\begin{array}{c|c}
\gamma^{-1}\left(1-\boldsymbol{u}^{T} \boldsymbol{w}\right)^{-1} & \boldsymbol{u}^{T}\left(I-\boldsymbol{w} \boldsymbol{u}^{T}\right)^{-1} \Sigma^{-1} \\
\hline \gamma^{-1}\left(1-\boldsymbol{u}^{T} \boldsymbol{w}\right)^{-1} \boldsymbol{w} & \left(I-\boldsymbol{w} \boldsymbol{u}^{T}\right)^{-1} \Sigma^{-1}
\end{array}\right] .
\end{gathered}
$$

These three ways of parameterizing the $4 \times 4$ matrix $M$ are completely equivalent and mathematically acceptable, and which one we adopt is simply a matter of taste. (It is easy to explicitly carry out the matrix multiplications and so to verify that $M M^{-1}=I$.) 


\subsubsection{Aether frame and moving frame}

By renouncing the relativity principle - that is, saying that the laws of physics are not the same in all the inertial reference frames - we have introduced a preferred rest frame, that we shall call aether.

We shall indicate the aether frame $F$ with coordinates $X$, and a generic moving frame $\bar{F}$ with coordinates $\bar{X}$. Then for definiteness we will choose $M$ to map from the aether frame to the moving frame, and $M^{-1}$ to map from the moving frame to the aether frame, so that

$$
\bar{X}=M X ; \quad X=M^{-1} \bar{X} .
$$

As we have not specified any particular characteristics that can, at this stage, distinguish between the aether and any other reference frame, choosing which of the frames is the aether and which is moving is merely a matter of convention.

We shall now rename things slightly and shall henceforth adopt the specific convention and nomenclature that:

$$
\begin{gathered}
M=\left[\begin{array}{c|c}
\gamma & -\gamma \boldsymbol{u}^{T} \\
\hline-\Sigma \boldsymbol{v} & \Sigma
\end{array}\right] ; \\
M^{-1}=\left[\begin{array}{c|c}
\gamma^{-1}\left(1-\boldsymbol{u}^{T} \boldsymbol{v}\right)^{-1} & \boldsymbol{u}^{T}\left(I-\boldsymbol{v} \boldsymbol{u}^{T}\right)^{-1} \Sigma^{-1} \\
\hline \gamma^{-1}\left(1-\boldsymbol{u}^{T} \boldsymbol{v}\right)^{-1} \boldsymbol{v} & \left(I-\boldsymbol{v} \boldsymbol{u}^{T}\right)^{-1} \Sigma^{-1}
\end{array}\right] .
\end{gathered}
$$

With these conventions both $\gamma$ and $\Sigma$ are dimensionless, while $\boldsymbol{v}$ has the dimensions of velocity, and $\boldsymbol{u}$ has dimensions of "slowness" $=1 /$ (velocity). It is again easy to verify that $M M^{-1}=I$. It is important to stress that the factor $\gamma$ must not be confused with the Lorentz factor of special relativity - at least as long as we do not impose isotropy and the principle of relativity. We can simplify the notation by noticing that (see appendix A.3

$$
\boldsymbol{u}^{T}\left(I-\boldsymbol{v} \boldsymbol{u}^{T}\right)^{-1}=\left(1-\boldsymbol{u}^{T} \boldsymbol{v}\right)^{-1} \boldsymbol{u}^{T}=(1-\boldsymbol{u} \cdot \boldsymbol{v})^{-1} \boldsymbol{u}^{T},
$$

and so the matrix $M$ is still

$$
M=\left[\begin{array}{c|c}
\gamma & -\gamma \boldsymbol{u}^{T} \\
\hline-\Sigma \boldsymbol{v} & \Sigma
\end{array}\right]
$$

while the inverse becomes:

$$
M^{-1}=\left[\begin{array}{c|c}
\gamma^{-1}(1-\boldsymbol{u} \cdot \boldsymbol{v})^{-1} & (1-\boldsymbol{u} \cdot \boldsymbol{v})^{-1} \boldsymbol{u}^{T} \Sigma^{-1} \\
\hline \gamma^{-1}(1-\boldsymbol{u} \cdot \boldsymbol{v})^{-1} \boldsymbol{v} & (I-\boldsymbol{v} \otimes \boldsymbol{u})^{-1} \Sigma^{-1}
\end{array}\right] .
$$


Note that there is a kinematic singularity if $\boldsymbol{u} \cdot \boldsymbol{v}=1$; in the particular case of special relativity this would correspond to an infinite boost to a frame traveling at lightspeed. But the possible occurrence of these kinematic singularities is a much more general phenomenon, and is not limited to special relativity. Indeed, since with our conventions the matrix $M$ factorizes

$$
M=\left[\begin{array}{c|c}
\gamma & \mathbf{0}^{T} \\
\hline \mathbf{0} & \Sigma
\end{array}\right]\left[\begin{array}{c|c}
1 & -\boldsymbol{u}^{T} \\
\hline-\boldsymbol{v} & I
\end{array}\right],
$$

we see that

$$
\operatorname{det}(M)=\gamma \operatorname{det}(\Sigma) \operatorname{det}\left[\begin{array}{c|c}
1 & -\boldsymbol{u}^{T} \\
\hline-\boldsymbol{v} & I
\end{array}\right]=\gamma \operatorname{det}(\Sigma)[1-\boldsymbol{u} \cdot \boldsymbol{v}] .
$$

So the existence of the kinematic singularity is equivalent to the non-invertibility of the transformation matrix, a possibility that should (generically) be excluded on physical grounds.

\section{Reciprocal velocities}

We shall now see that, with the set of transformation we have introduced in (4.17), we can start to observe some of the peculiarities that characterise the physics without the relativity principle.

For instance, an object that is at rest in the moving frame follows the worldline

$$
\bar{X}=\left(\begin{array}{c}
\bar{t} \\
\mathbf{0}
\end{array}\right)
$$

which in the aether frame coordinates maps into

$$
X=M^{-1} \bar{X}=\bar{t} \gamma^{-1}(1-\boldsymbol{u} \cdot \boldsymbol{v})^{-1}\left(\begin{array}{l}
1 \\
\boldsymbol{v}
\end{array}\right)
$$

This implies $\mathbf{x}=\boldsymbol{v} t$. That is, with these conventions the moving frame has 3 -velocity $\boldsymbol{v}_{\text {moving }}=\boldsymbol{v}$ as viewed by the aether, and this is our physical interpretation of the parameter $\boldsymbol{v}$ appearing in the matrix $M$. But what about the aether frame as seen by the moving frame? An object at rest in the aether frame follows the worldline

$$
X=\left(\begin{array}{l}
t \\
\mathbf{0}
\end{array}\right),
$$

which in the moving frame coordinates maps into

$$
\bar{X}=M X=t\left(\begin{array}{c}
\gamma \\
-\Sigma \boldsymbol{v}
\end{array}\right)
$$


This implies $\overline{\mathbf{x}}=-(\Sigma \boldsymbol{v} / \gamma) \bar{t}$. That is, as viewed in the moving frame, the aether is moving with 3-velocity

$$
\boldsymbol{v}_{\text {aether }}=-\frac{\Sigma \boldsymbol{v}}{\gamma} .
$$

Note that $\boldsymbol{v}_{\text {moving }}$ and $\boldsymbol{v}_{\text {aether }}$ are generally not equal-but-opposite velocities. In fact, without additional assumptions, in the general case they need not even be collinear, a result that is completely counterintuitive from the point of view of, for example, special relativity.

\subsubsection{Transformation of 3-velocity}

We now want to explore how the transformations of 3 -velocities are modified with respect to classical newtonian kinematics and special relativity.

From $\bar{X}=M X$ we have $d \bar{X}=M d X$, whence with the conventions adopted above we see

$$
d \bar{t}=\gamma(d t-\boldsymbol{u} \cdot d \mathbf{x}) ; \quad d \overline{\mathbf{x}}=\Sigma(d \mathbf{x}-\boldsymbol{v} d t)
$$

so that

$$
\dot{\overline{\mathbf{x}}} \equiv \frac{d \overline{\mathbf{x}}}{d \bar{t}}=\frac{\Sigma(\dot{\mathbf{x}}-\boldsymbol{v})}{\gamma(1-\boldsymbol{u} \cdot \dot{\mathbf{x}})} .
$$

This is the general combination of velocities rule. Specifically one can easily see that it is a natural generalisation of the usual special relativistic combination of velocities, with current conventions being chosen to make this transformation as simple as possible. Note in particular that an object at rest in the aether frame, with $\dot{\mathbf{x}}=\mathbf{0}$, moves at 3 -velocity $-\Sigma \boldsymbol{v} / \gamma$ in the moving frame, while an object at rest in the moving frame, with $\dot{\overline{\mathbf{x}}}=\mathbf{0}$, moves at 3-velocity $\boldsymbol{v}$ in the aether frame.

Similarly, from $d X=M^{-1} d \bar{X}$ we have

$$
d t=\gamma^{-1}(1-\boldsymbol{u} \cdot \boldsymbol{v})^{-1} d \bar{t}+(1-\boldsymbol{u} \cdot \boldsymbol{v})^{-1} \boldsymbol{u}^{T} \Sigma^{-1} d \overline{\mathbf{x}},
$$

and

$$
d \mathbf{x}=(I-\boldsymbol{v} \otimes \boldsymbol{u})^{-1} \Sigma^{-1} d \overline{\mathbf{x}}+\gamma^{-1}(1-\boldsymbol{u} \cdot \boldsymbol{v})^{-1} \boldsymbol{v} d \bar{t},
$$

so that

$$
\dot{\mathbf{x}} \equiv \frac{d \mathbf{x}}{d t}=\frac{(I-\boldsymbol{v} \otimes \boldsymbol{u})^{-1} \Sigma^{-1} \dot{\overline{\mathbf{x}}}+\gamma^{-1}(1-\boldsymbol{u} \cdot \boldsymbol{v})^{-1} \boldsymbol{v}}{\gamma^{-1}(1-\boldsymbol{u} \cdot \boldsymbol{v})^{-1}+(1-\boldsymbol{u} \cdot \boldsymbol{v})^{-1} \boldsymbol{u}^{T} \Sigma^{-1} \dot{\dot{\mathbf{x}}}} .
$$

We can simplify this to obtain

$$
\dot{\mathbf{x}}=\frac{\gamma(1-\boldsymbol{u} \cdot \boldsymbol{v})(I-\boldsymbol{v} \otimes \boldsymbol{u})^{-1} \Sigma^{-1} \dot{\overline{\mathbf{x}}}+\boldsymbol{v}}{1+\gamma \boldsymbol{u}^{T} \Sigma^{-1} \dot{\overline{\mathbf{x}}}} .
$$

But (see appendix A.3

$$
(1-\boldsymbol{u} \cdot \boldsymbol{v})(I-\boldsymbol{v} \otimes \boldsymbol{u})^{-1}=(1-\boldsymbol{u} \cdot \boldsymbol{v}) I+\boldsymbol{v} \otimes \boldsymbol{u},
$$


and so

$$
\dot{\mathbf{x}}=\frac{\gamma[(1-\boldsymbol{u} \cdot \boldsymbol{v}) I+\boldsymbol{v} \otimes \boldsymbol{u}] \Sigma^{-1} \dot{\overline{\mathbf{x}}}+\boldsymbol{v}}{1+\gamma \boldsymbol{u}^{T} \Sigma^{-1} \dot{\overline{\mathbf{x}}}} .
$$

Finally

$$
\dot{\mathbf{x}}=\frac{\gamma(1-\boldsymbol{u} \cdot \boldsymbol{v}) \Sigma^{-1} \dot{\overline{\mathbf{x}}}}{1+\gamma \boldsymbol{u}^{T} \Sigma^{-1} \dot{\overline{\mathbf{x}}}}+\boldsymbol{v} .
$$

This last formula seems to be the best one can do. Attempting to change conventions to simplify this particular formula leads to problems elsewhere. Note in particular that with the conventions we have chosen something at rest in the moving frame, so that $\dot{\overline{\mathbf{x}}}=0$, moves at velocity $\boldsymbol{v}$ in the aether frame.

\subsubsection{Groupoid/pseudogroup structure}

Physically the matrix $M$ need not be a function of $\boldsymbol{v}$ only - it can also depend on the orientation of the moving inertial frame with respect to the preferred frame, and worse the quantities $\gamma, \boldsymbol{u}$, and $\Sigma$, are (potentially) free parameters in their own right. It is useful to write $M(\bar{F})$, to emphasise that the matrix $M(\bar{F})$ is potentially a function of all the parameters characterizing the moving inertial frame $\bar{F}$. (We could furthermore write the various pieces of $M(\bar{F})$ as $\gamma(\bar{F}), \boldsymbol{v}(\bar{F}), \boldsymbol{u}(\bar{F})$, and $\Sigma(\bar{F})$; while technically more correct, this is so cumbersome as to be impracticable, and the frame dependence of these quantities will always be implicitly understood.)

In addition, one should keep in mind that in general the transformation matrices $M(\bar{F})$ could also depend on the internal structure of the particular type of rulers and clocks one is using; it is only for situations of very high symmetry — essentially amounting to adoption of the relativity principle - that the notion of time and distance can be abstracted to have a meaning that is independent of the internal structure of one's choice of clocks and rulers.

Note that in general the set $\{M(\bar{F})\}$, (where $M(\bar{F})$ is the transformation matrix from the aether inertial frame $F$ to the moving inertial frame $\bar{F}$ ), need not form a group; and similarly the set $\left\{M^{-1}(\bar{F})\right\}$ need not form a group. There is no need for these sets to be closed under matrix multiplication. Nor are these sets generally closed under matrix inversion. There does not seem to be any specialized mathematical terminology for such objects - they are not semigroups, they are not groupoids, they are not pseudogroups, they are not monoids, they are not cosets, they are not magmas; they are just sets of matrices.

However to transform from one arbitrary inertial frame $F_{1}$ to another arbitrary inertial frame $F_{2}$, figure 4.1 , the appropriate transformation is

$$
M\left(F_{2}, F_{1}\right)=M\left(F_{2}\right) M\left(F_{1}\right)^{-1} .
$$




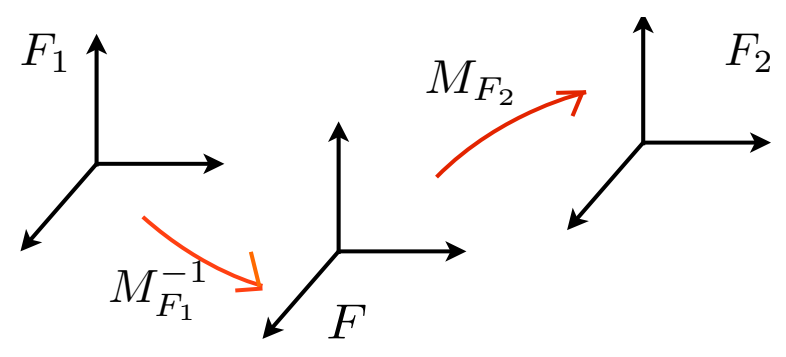

Figure 4.1: A transformation from an arbitrary inertial frame $F_{1}$ to another arbitrary inertial frame $F_{2}$. Since we know how to perform a transformation only between the aether frame $F$ and any other inertial frame, we need to transform from $F_{1}$ to $F$ first, using the transformation matrix $M_{F_{1}}^{-1}$ and afterwards perform the transformation between $F$ and $F_{2}$ using the transformation matrix $M_{F_{2}}$.

The set $\left\{M\left(F_{2}, F_{1}\right)\right\}=\left\{M\left(F_{2}\right) M\left(F_{1}\right)^{-1}\right\}$ certainly forms a groupoid/pseudogroup, in the sense that the set is closed under the restricted set of compositions (so-called partial-products) of the form

$$
M\left(F_{3}, F_{2}\right) M\left(F_{2}, F_{1}\right)=M\left(F_{3}, F_{1}\right) .
$$

(The relevant mathematical terminology is not 100\% standardized, and different sources prefer to call this mathematical structure either a groupoid or a pseudogroup.) Note that $M(F, F)=I$, so an identity certainly exists, and that $M\left(F_{2}, F_{1}\right)^{-1}=$ $M\left(F_{1}, F_{2}\right)$ so that inverses also exist. Associativity is automatic because matrix multiplication is associative. In general this is the most you can say. The technical difference between a group and a groupoid/pseudogroup is in this context extremely important. It is this technical mathematical distinction that ultimately allows us to side-step the usual von Ignatowsky theorems (and their variants) that under normal circumstances lead almost uniquely to the Lorentz group or the Galileo group - the physics reason for this extra generality is because while we have assumed linearity of the transformations we have not assumed the relativity principle. (Nor have we at this stage assumed isotropy, but that is not important for this particular issue.)

The key physics point is that without the relativity principle $M\left(F_{1}, F_{2}\right)$ need not depend merely on the relative velocities between the frames $F_{1}$ and $F_{2}$, but is 
instead allowed to depend on the separate absolute velocities of the frames $F_{1}$ and $F_{2}$.

\subsection{Transformations of energy and momentum}

Defining energy and momentum, as opposed to purely kinematical notions of velocity and position, requires at least some notion of dynamics. Pick some arbitrary but fixed inertial frame. To study dynamics in that frame, one should at a minimum be able to formulate all three of Newton's laws, and one should at a minimum be able to formulate notions of energy and momentum. Since both Hamiltonian and Lagrangian dynamics are essentially just a reformulation of Newton's three laws, and implicitly of the notions of energy and momentum, one should in each individual inertial frame be able to develop some version of Hamiltonian/Lagrangian dynamics. (Furthermore, we note that any attempt at developing usual notions of quantum physics requires one to first develop Hamiltonian/ Lagrangian dynamics - either to insert into the path integral formulation, or to serve as the basis for a quantum Hamiltonian underlying the Schroedinger equation.) The natural question then arises as to how the Hamiltonian/Lagrangian dynamics in different inertial frames are related to each other 11 Understanding this will tell us how energy and momentum in different inertial frames are related to each other.

\subsubsection{Defining energy and momentum}

Ignoring interactions for now, in view of the homogeneity of space-time we shall assume that each particle has associated with it some Lagrangian $L(\dot{\mathbf{x}})$ which leads to a momentum $\boldsymbol{p}(\dot{\mathbf{x}})=\partial L / \partial \dot{\mathbf{x}}$, and hence to a Hamiltonian $H(\boldsymbol{p})$, which we shall typically just write as $E(\boldsymbol{p})$. Because of space-time homogeneity and the Hamiltonian/Lagrangian framework, Noether's theorem implies energy and momentum conservation:

$$
\sum_{\text {in }} E_{i}=\sum_{\text {out }} E_{i} ; \quad \quad \sum_{\text {in }} \boldsymbol{p}_{i}=\sum_{\text {out }} \boldsymbol{p}_{i}
$$

Now the inertial equations $\ddot{\mathbf{x}}=0$ will be satisfied for any arbitrary $L(\dot{\mathbf{x}})$. (Note the absence of any explicit $t$ or $\mathbf{x}$ dependence.) To operationally determine a specific

\footnotetext{
${ }^{1}$ Some interesting questions can nevertheless be dealt with by working within a fixed but arbitrary inertial frame and not worrying about the transformation rules. For instance, scattering and decay thresholds in Lorentz violating theories can usefully be dealt with in such a manner, see for instance $87,89,158,165,198,205,210,211$. We shall present our considerations about this matter in chapter 5
} 
$L(\dot{\mathbf{x}})$ that can usefully characterize a specific particle, one will want to perform a large number of collisions at various 3 -velocities, compare input and output states, and data-fit to extract suitable $E_{i}(\dot{\mathbf{x}})$ and $\boldsymbol{p}_{i}(\dot{\mathbf{x}})$ corresponding to the various particles in your universe of discourse. Once this is done you can build a model for the $L_{i}(\dot{\mathbf{x}})$ using

$$
L_{i}(\dot{\mathbf{x}})=L_{i}(\mathbf{0})+\int_{\mathbf{0}}^{\dot{\mathbf{x}}} \boldsymbol{p}(\dot{\tilde{\mathbf{x}}}) \cdot d \dot{\tilde{\mathbf{x}}}
$$

Note that, in modelling the $\boldsymbol{p}_{i}(\dot{\mathbf{x}})$, one would have to take into account the consistency condition $\nabla_{\dot{\mathbf{x}}} \times \boldsymbol{p}_{i}(\dot{\mathbf{x}})$ required for this construction to be path independent in velocity space.

But even after one has done this, the construction cannot be unique - for any set of constants $\epsilon_{i}$ and $\boldsymbol{\pi}_{i}$ such that $\sum_{\text {in }} \epsilon_{i}=\sum_{\text {out }} \epsilon_{i}$ and $\sum_{\text {in }} \boldsymbol{\pi}_{i}=\sum_{\text {out }} \boldsymbol{\pi}_{i}$ we see that the assignments

$$
E_{i} \leftrightarrow E_{i}+\epsilon_{i} ; \quad \boldsymbol{p}_{i} \leftrightarrow \boldsymbol{p}_{i}+\boldsymbol{\pi}_{i}
$$

are physically indistinguishable. But that means the Lagrangians

$$
L_{i}(\dot{\mathbf{x}}) \leftrightarrow L_{i}(\dot{\mathbf{x}})-\epsilon_{i}+\boldsymbol{\pi}_{i} \cdot \dot{\mathbf{x}}
$$

are physically indistinguishable. In terms of the action this means the quantities

$$
S_{i}=\int L_{i}(\dot{\mathbf{x}}) d t \leftrightarrow S_{i}=\int L_{i}(\dot{\mathbf{x}}) d t-\epsilon_{i}\left(t_{F}-t_{I}\right)+\boldsymbol{\pi}_{i} \cdot\left(\mathbf{x}_{F}-\mathbf{x}_{I}\right)
$$

are physically indistinguishable — which is physically and mathematically obvious in view of the fact that the two actions differ only by boundary terms. This intrinsic ambiguity in the definition of energy and momentum will (perhaps unfortunately) turn out to be important. One could try to resolve these ambiguities in a number of different ways:

- For instance, the ambiguity in momentum could be fixed by setting the momentum at zero velocity to be zero: $\boldsymbol{p}(\dot{\mathbf{x}}=\mathbf{0})=\mathbf{0}$. Sometimes this works well, sometimes it does not.

- The ambiguity in energy is equivalent to an ambiguity in rest energy $E(\dot{\mathbf{x}}=\mathbf{0})$; attempting to set the rest energy to zero is often severely problematic.

In general it is best to keep this freedom available in the calculation as long as possible. 


\subsubsection{Affine versus linear transformations}

What can we now say about energy and momentum, and their transformation properties, using only linearity of the transformations between inertial frames? (Recall that we very specifically do not assume isotropy or any form of the relativity principle.)

Consider a single particle, but multiple inertial frames. To even begin to talk about energy and momentum, in each frame one must be able to set up a suitable Lagrangian and Hamiltonian, and there should be some as yet unspecified relationship between the Lagrangians and Hamiltonians in these distinct inertial frames. Furthermore, extrema of the action as calculated in one inertial frame must coincide with extrema of the action calculated in any other inertial frame.

That is, in complete generality we should demand that for any two inertial frames the action calculated in these frames should be equal up to boundary terms, and in each individual frame we know the action is ambiguous up to boundary terms. In view of the groupoid structure of the transformations between inertial frames there is no loss of generality in considering one moving frame $\bar{F}$ plus the aether frame $F$ for which we can write

$$
\int \bar{L} d \bar{t}+(\text { boundary terms })=\int L d t+\text { (boundary terms). }
$$

In view of our previous discussion this implies

$$
\int\{\bar{L}-\bar{\epsilon}+\overline{\boldsymbol{\pi}} \cdot(d \overline{\mathbf{x}} / d \bar{t})\} d \bar{t}=\int\{L-\epsilon+\boldsymbol{\pi} \cdot(d \mathbf{x} / d t)\} d t .
$$

But since $L=-(E-\boldsymbol{p} \cdot \dot{\mathbf{x}})$ this implies

$$
\int\{(\bar{E}+\bar{\epsilon})-(\overline{\boldsymbol{p}}+\overline{\boldsymbol{\pi}}) \cdot(d \overline{\mathbf{x}} / d \bar{t})\} d \bar{t}=\int\{(E+\epsilon)-(\boldsymbol{p}+\boldsymbol{\pi}) \cdot(d \mathbf{x} / d t)\} d t .
$$

Therefore

$$
(\bar{E}+\bar{\epsilon}) d \bar{t}-(\overline{\boldsymbol{p}}+\overline{\boldsymbol{\pi}}) \cdot d \overline{\mathbf{x}}=(E+\epsilon) d t-(\boldsymbol{p}+\boldsymbol{\pi}) \cdot d \mathbf{x} .
$$

So now in complete generality we have

$$
\left(\bar{E}+\bar{\epsilon},-\overline{\boldsymbol{p}}^{T}-\overline{\boldsymbol{\pi}}^{T}\right)\left(\begin{array}{c}
d \bar{t} \\
d \overline{\mathbf{x}}
\end{array}\right)=\left(E+\epsilon,-\boldsymbol{p}^{T}-\boldsymbol{\pi}^{T}\right)\left(\begin{array}{c}
d t \\
d \mathbf{x}
\end{array}\right) .
$$

But

$$
\left(\begin{array}{c}
d \bar{t} \\
d \overline{\mathbf{x}}
\end{array}\right)=M\left(\begin{array}{c}
d t \\
d \mathbf{x}
\end{array}\right)
$$

therefore implying both

$$
\left(E+\epsilon,-\boldsymbol{p}^{T}-\boldsymbol{\pi}^{T}\right)=\left(\bar{E}+\bar{\epsilon},-\overline{\boldsymbol{p}}^{T}-\overline{\boldsymbol{\pi}}^{T}\right) M,
$$


and

$$
\left(\bar{E}+\bar{\epsilon},-\overline{\boldsymbol{p}}^{T}-\overline{\boldsymbol{\pi}}^{T}\right)=\left(E+\epsilon,-\boldsymbol{p}^{T}-\boldsymbol{\pi}^{T}\right) M^{-1} .
$$

These are affine transformation laws for energy and momentum, (that is, linear plus an inhomogeneous offset), with the affine piece only depending on the intrinsic ambiguities $\left(\epsilon,-\boldsymbol{\pi}^{T}\right)$ and $\left(\bar{\epsilon},-\overline{\boldsymbol{\pi}}^{T}\right)$ in the energy and momentum. Note that $P=$ $\left(E,-\boldsymbol{p}^{T}\right)$ transforms in the dual space to 4-position $X=\left(t, \mathbf{x}^{T}\right)^{T}$. To be explicit about this

$$
E \rightarrow \bar{E}=\frac{E-\boldsymbol{p}^{T} \boldsymbol{v}}{\gamma\left(1-\boldsymbol{u}^{T} \boldsymbol{v}\right)}+\frac{\epsilon-\boldsymbol{\pi}^{T} \boldsymbol{v}}{\gamma\left(1-\boldsymbol{u}^{T} \boldsymbol{v}\right)}-\bar{\epsilon}
$$

and

$$
\boldsymbol{p} \rightarrow \overline{\boldsymbol{p}}=\left(\Sigma^{-1}\right)^{T}\left(I-\boldsymbol{u} \boldsymbol{v}^{T}\right)^{-1}(\boldsymbol{p}-E \boldsymbol{u})+\left(\Sigma^{-1}\right)^{T}\left(I-\boldsymbol{u} \boldsymbol{v}^{T}\right)^{-1}(\boldsymbol{\pi}-\epsilon \boldsymbol{u})-\overline{\boldsymbol{\pi}} .
$$

In terms of dot and tensor products we can rewrite this as

$$
E \rightarrow \bar{E}=\frac{E-\boldsymbol{p} \cdot \boldsymbol{v}}{\gamma(1-\boldsymbol{u} \cdot \boldsymbol{v})}+\frac{\epsilon-\boldsymbol{\pi} \cdot \boldsymbol{v}}{\gamma(1-\boldsymbol{u} \cdot \boldsymbol{v})}-\bar{\epsilon}
$$

and

$$
\boldsymbol{p} \rightarrow \overline{\boldsymbol{p}}=\left(\Sigma^{-1}\right)^{T}(I-\boldsymbol{u} \otimes \boldsymbol{v})^{-1}(\boldsymbol{p}-E \boldsymbol{u})+\left(\Sigma^{-1}\right)^{T}(I-\boldsymbol{u} \otimes \boldsymbol{v})^{-1}(\boldsymbol{\pi}-\epsilon \boldsymbol{u})-\overline{\boldsymbol{\pi}} .
$$

(One can now begin to see how the Lorentz and Galilean transformations might emerge as special cases of this very general result.) The inverse transformations are somewhat simpler

$$
\bar{E} \rightarrow E=\gamma \bar{E}+\overline{\boldsymbol{p}}^{T} \Sigma \boldsymbol{v}+\gamma \bar{\epsilon}+\overline{\boldsymbol{\pi}}^{T} \Sigma \boldsymbol{v}-\epsilon
$$

and

$$
\overline{\boldsymbol{p}} \rightarrow \boldsymbol{p}=\gamma \bar{E} \boldsymbol{u}+\Sigma^{T} \overline{\boldsymbol{p}}+\gamma \bar{\epsilon} \boldsymbol{u}+\Sigma^{T} \overline{\boldsymbol{\pi}}-\boldsymbol{\pi} .
$$

Suppose we now consider the same particle at two different 3 -velocities, but working with the same two inertial frames $F$ and $\bar{F}$; then in terms of energy and momentum differences, we can write a homogeneous linear transformation law of the form

$$
\left(\left[\bar{E}_{1}-\bar{E}_{2}\right],-\left[\overline{\boldsymbol{p}}_{1}-\overline{\boldsymbol{p}}_{2}\right]^{T}\right)=\left(\left[E_{1}-E_{2}\right],-\left[\boldsymbol{p}_{1}-\boldsymbol{p}_{2}\right]^{T}\right) M^{-1}
$$

That is:

$$
\Delta E \rightarrow \Delta \bar{E}=\frac{\Delta E-\Delta \boldsymbol{p} \cdot \boldsymbol{v}}{\gamma(1-\boldsymbol{u} \cdot \boldsymbol{v})}
$$

and

$$
\Delta \boldsymbol{p} \rightarrow \Delta \overline{\boldsymbol{p}}=\left(\Sigma^{-1}\right)^{T}(I-\boldsymbol{u} \otimes \boldsymbol{v})^{-1}(\Delta \boldsymbol{p}-\Delta E \boldsymbol{u})
$$


We need to compare the same particle at two different velocities, since otherwise there is no particular reason for the $\left(\epsilon,-\boldsymbol{\pi}^{T}\right)$ and $\left(\bar{\epsilon},-\overline{\boldsymbol{\pi}}^{T}\right)$ to be the same for the two situations. Note that for two otherwise identical particles one could in principle choose differing values for the parameters $\left(\epsilon,-\boldsymbol{\pi}^{T}\right)$ and $\left(\bar{\epsilon},-\overline{\boldsymbol{\pi}}^{T}\right)$, thereby making them distinguishable. This does not appear to be what happens in our universe, so we shall assume that the quantities $\left(\epsilon,-\boldsymbol{\pi}^{T}\right)$ and $\left(\bar{\epsilon},-\overline{\boldsymbol{\pi}}^{T}\right)$, while they might depend on the inertial frame one is working in, are at least universal for any particular particle species.

Note that in terms of energy-momentum differences the inverse transformations are

$$
\Delta \bar{E} \rightarrow \Delta E=\gamma \Delta \bar{E}+\Delta \overline{\boldsymbol{p}}^{T} \Sigma \boldsymbol{v}
$$

and

$$
\Delta \overline{\boldsymbol{p}} \rightarrow \Delta \boldsymbol{p}=\gamma \Delta \bar{E} \boldsymbol{u}+\Sigma^{T} \Delta \overline{\boldsymbol{p}}
$$

As a consistency check on the general formalism we can readily verify that these energy-momentum transformation laws are compatible with, and permit us to recover, the purely kinematical velocity combination rules. See appendix A.4 for details.

\subsubsection{Summary (Energy-momentum)}

Let us now summarise the results we have obtained so far for the transformation of energy and momentum in order to make the next stages more understandable. We have seen that for each individual particle species we have

$$
E \rightarrow \bar{E}=\frac{E-\boldsymbol{p} \cdot \boldsymbol{v}}{\gamma(1-\boldsymbol{u} \cdot \boldsymbol{v})}+\frac{\epsilon-\boldsymbol{\pi} \cdot \boldsymbol{v}}{\gamma(1-\boldsymbol{u} \cdot \boldsymbol{v})}-\bar{\epsilon}
$$

and

$$
\boldsymbol{p} \rightarrow \overline{\boldsymbol{p}}=\left(\Sigma^{-1}\right)^{T}(I-\boldsymbol{u} \otimes \boldsymbol{v})^{-1}(\boldsymbol{p}-E \boldsymbol{u})+\left(\Sigma^{-1}\right)^{T}(I-\boldsymbol{u} \otimes \boldsymbol{v})^{-1}(\boldsymbol{\pi}-\epsilon \boldsymbol{u})-\overline{\boldsymbol{\pi}}
$$

while the inverse transformations are

$$
\bar{E} \rightarrow E=-\epsilon+\gamma \bar{E}+\overline{\boldsymbol{p}} \cdot(\Sigma \boldsymbol{v})+\gamma \bar{\epsilon}+\overline{\boldsymbol{\pi}} \cdot(\Sigma \boldsymbol{v}),
$$

and

$$
\overline{\boldsymbol{p}} \rightarrow \boldsymbol{p}=-\boldsymbol{\pi}+\gamma \bar{E} \boldsymbol{u}+\Sigma^{T} \overline{\boldsymbol{p}}+\gamma \bar{\epsilon} \boldsymbol{u}+\Sigma^{T} \overline{\boldsymbol{\pi}}
$$

We have a certain amount of freedom to choose $\epsilon$ and $\boldsymbol{\pi}$, and $\bar{\epsilon}$ and $\overline{\boldsymbol{\pi}}$. One obvious choice would be to always make the transformation laws linear; however as we shall soon see this is not always the best thing to do. 


\subsection{Examples}

Now that we have found the general rules for the transformation of energy and momentum between the aether and an arbitrary (inertial) frame, we shall consider the very standard cases of Galilean invariance and Lorentz invariance - comparing affine and linear transformation laws for energy-momentum for these two cases - to understand how to properly choose the offset parameters in a more general scenario.

\subsubsection{Galileo group (affine version)}

For Galilean kinematics we have

$$
M=\left[\begin{array}{c|c}
1 & \mathbf{0}^{T} \\
\hline-\boldsymbol{v} & I
\end{array}\right],
$$

so

$$
\bar{t}=t ; \quad \overline{\mathbf{x}}=\mathbf{x}-\boldsymbol{v} t ; \quad \dot{\overline{\mathbf{x}}}=\dot{\mathbf{x}}-\boldsymbol{v} .
$$

Now one natural choice is to choose the particularly simple and standard Lagrangians (we are considering non-interacting particles)

$$
L=\frac{1}{2} m\|\dot{\mathbf{x}}\|^{2} ; \quad \bar{L}=\frac{1}{2} m\|\dot{\mathbf{x}}\|^{2} .
$$

(We shall soon see that there are also other choices one can make.) Then

$$
\bar{L}=\frac{1}{2} m\|\dot{\mathbf{x}}\|^{2}=\frac{1}{2} m\|\dot{\mathbf{x}}-\boldsymbol{v}\|^{2}=\frac{1}{2} m\|\dot{\mathbf{x}}\|^{2}-m \boldsymbol{v} \cdot \dot{\mathbf{x}}+\frac{1}{2} m\|\boldsymbol{v}\|^{2} .
$$

That is

$$
\bar{L}=L+\frac{1}{2} m\|\boldsymbol{v}\|^{2}-m \boldsymbol{v} \cdot \dot{\mathbf{x}} .
$$

Now note

$$
\begin{gathered}
\overline{\boldsymbol{p}}=m \dot{\mathbf{x}}-m \boldsymbol{v}=m \dot{\overline{\mathbf{x}}} \\
\bar{H}=\overline{\boldsymbol{p}} \cdot \dot{\overline{\mathbf{x}}}-\bar{L}=\frac{\|\overline{\boldsymbol{p}}\|^{2}}{2 m} .
\end{gathered}
$$

So working explicitly, with these particular conventions, we have affine transformations for energy-momentum:

$$
\begin{gathered}
\bar{E}=E-\boldsymbol{p} \cdot \boldsymbol{v}+\frac{1}{2} m\|\boldsymbol{v}\|^{2} \\
\overline{\boldsymbol{p}}=\boldsymbol{p}-m \boldsymbol{v} .
\end{gathered}
$$

In contrast, working directly from the general transformation laws derived above, and taking $\gamma=1, \boldsymbol{u}=0$, and $\Sigma=I$, we have

$$
E \rightarrow \bar{E}=-\bar{\epsilon}+E-\boldsymbol{p} \cdot \boldsymbol{v}+[\epsilon-\boldsymbol{\pi} \cdot \boldsymbol{v}]=E-\boldsymbol{p} \cdot \boldsymbol{v}+\frac{1}{2} m\|\boldsymbol{v}\|^{2},
$$


and

$$
\boldsymbol{p} \rightarrow \overline{\boldsymbol{p}}=-\overline{\boldsymbol{\pi}}+\boldsymbol{p}+\boldsymbol{\pi}=\boldsymbol{p}-m \boldsymbol{v},
$$

from which we deduce that this particular way of implementing Galilean mechanics corresponds to the choices

$$
\bar{\epsilon}=-\frac{1}{2} m\|\boldsymbol{v}\|^{2} ; \quad \overline{\boldsymbol{\pi}}=m \boldsymbol{v} ; \quad \epsilon=0 ; \quad \boldsymbol{\pi}=0 .
$$

(Remember that by convention $\bar{F}$ is the moving frame while $F$ is the "aether" frame. Note that it is the quantities $\{\bar{\epsilon}, \overline{\boldsymbol{\pi}}\}$ associated with the moving frame that are nonzero, and that these quantities depend on the velocity $\boldsymbol{v}$ of the moving frame.) The inverse transformations are

$$
\bar{E} \rightarrow E=-\epsilon+\bar{E}+\overline{\boldsymbol{p}} \cdot \boldsymbol{v}+\bar{\epsilon}+\overline{\boldsymbol{\pi}} \cdot \boldsymbol{v}=\bar{E}+\overline{\boldsymbol{p}} \cdot \boldsymbol{v}+\frac{1}{2} m\|\boldsymbol{v}\|^{2},
$$

and

$$
\overline{\boldsymbol{p}} \rightarrow \boldsymbol{p}=-\boldsymbol{\pi}+\overline{\boldsymbol{p}}+\overline{\boldsymbol{\pi}}=\overline{\boldsymbol{p}}+m \boldsymbol{v} .
$$

This is the "usual" way of doing Galilean dynamics, which unavoidably leads to affine transformations for energy and momentum.

A somewhat subtle message to be taken from the discussion is this: Since affine transformations arise so naturally in this extremely straightforward setting, it seems unlikely that the affine features of the energy-momentum transformations could always be completely eliminated in more general settings.

\subsubsection{Lorentz group (linear version)}

In this case the Lorentz transformations are

$$
M=\left[\begin{array}{c|c}
\gamma & -\gamma \boldsymbol{v}^{T} / c^{2} \\
\hline-\gamma \boldsymbol{v} & \gamma \mathbf{n} \otimes \mathbf{n}+[I-\mathbf{n} \otimes \mathbf{n}]
\end{array}\right]
$$

with $\boldsymbol{v}=v \mathbf{n}$ and $\gamma=\left(1-v^{2} / c^{2}\right)^{-1 / 2}$. The usual form of the relativistic Lagrangian is

$$
L=-m c^{2} \sqrt{1-\|\dot{\mathbf{x}}\|^{2} / c^{2}}
$$

so

$$
\boldsymbol{p}=\frac{m \dot{\mathbf{x}}}{\sqrt{1-\|\dot{\mathbf{x}}\|^{2} / c^{2}}} ; \quad H=\boldsymbol{p} \cdot \dot{\mathbf{x}}-L=\frac{m c^{2}}{\sqrt{1-\|\dot{\mathbf{x}}\|^{2} / c^{2}}}
$$

Furthermore

$$
\bar{L}=-m c^{2} \sqrt{1-\|\dot{\mathbf{x}}\|^{2} / c^{2}}
$$


so

$$
\overline{\boldsymbol{p}}=\frac{m \dot{\overline{\mathbf{x}}}}{\sqrt{1-\|\left.\dot{\overline{\mathbf{x}}}\right|^{2} / c^{2}}} ; \quad \bar{H}=\overline{\boldsymbol{p}} \cdot \dot{\overline{\mathbf{x}}}-\bar{L}=\frac{m c^{2}}{\sqrt{1-\|\left.\dot{\mathbf{x}}\right|^{2} / c^{2}}},
$$

and in fact

$$
\bar{L} d \bar{t}=L d t
$$

implying both $\epsilon=0$ and $\boldsymbol{\pi}=\mathbf{0}$, and $\bar{\epsilon}=0$ and $\overline{\boldsymbol{\pi}}=\mathbf{0}$. Then the energy-momentum transformations are just the usual linear Lorentz transformations

$$
\left(E,-\boldsymbol{p}^{T}\right)=\left(\bar{E},-\overline{\boldsymbol{p}}^{T}\right) M
$$

and

$$
\left(\bar{E},-\overline{\boldsymbol{p}}^{T}\right)=\left(E,-\boldsymbol{p}^{T}\right) M^{-1} .
$$

This is the standard way of implementing Lagrangian and Hamiltonian mechanics in the presence of Lorentz symmetry.

\subsubsection{Lorentz group (affine version)}

We could have chosen a slightly different normalization for $L$ and $H$. If we take

$$
L=m c^{2}\left\{1-\sqrt{1-\|\dot{\mathbf{x}}\|^{2} / c^{2}}\right\}
$$

then

$$
\boldsymbol{p}=\frac{m \dot{\mathbf{x}}}{\sqrt{1-\|\dot{\mathbf{x}}\|^{2} / c^{2}}} ; \quad H=\boldsymbol{p} \cdot \dot{\mathbf{x}}-L=\frac{m c^{2}}{\sqrt{1-\|\dot{\mathbf{x}}\|^{2} / c^{2}}}-m c^{2}
$$

Furthermore

$$
\bar{L}=m c^{2}\left\{1-\sqrt{1-\|\dot{\overline{\mathbf{x}}}\|^{2} / c^{2}}\right\}
$$

so

$$
\overline{\boldsymbol{p}}=\frac{m \dot{\overline{\mathbf{x}}}}{\sqrt{1-\|\dot{\overline{\mathbf{x}}}\|^{2} / c^{2}}} ; \quad \bar{H}=\overline{\boldsymbol{p}} \cdot \dot{\overline{\mathbf{x}}}-\bar{L}=\frac{m c^{2}}{\sqrt{1-\|\dot{\overline{\mathbf{x}}}\|^{2} / c^{2}}}-m c^{2} .
$$

In fact with this normalization

$$
\left[\bar{L}-m c^{2}\right] d \bar{t}=\left[L-m c^{2}\right] d t
$$

whence

$$
\bar{\epsilon}=m c^{2} ; \quad \overline{\boldsymbol{\pi}}=\mathbf{0} ; \quad \text { and } \quad \epsilon=m c^{2} ; \quad \boldsymbol{\pi}=\mathbf{0} .
$$

We can rephrase this in terms of the 4-velocities of the "aether" and moving frames as

$$
\left(\begin{array}{c}
\bar{\epsilon} \\
\overline{\boldsymbol{\pi}}
\end{array}\right)=m c^{2} \bar{V} ; \quad\left(\begin{array}{c}
\epsilon \\
\boldsymbol{\pi}
\end{array}\right)=m c^{2} V
$$


With these choices the energy-momentum transformations look slightly unusual. Taking $\boldsymbol{v} \| \boldsymbol{p}$ for simplicity (the non-collinear case does not teach us anything new) we now have

$$
E \rightarrow \bar{E}=\gamma\left(\left[m c^{2}+E\right]-\boldsymbol{p} \cdot \boldsymbol{v}\right)-m c^{2}
$$

and

$$
\boldsymbol{p} \rightarrow \overline{\boldsymbol{p}}=\gamma\left(\boldsymbol{p}-\left[m c^{2}+E\right] \boldsymbol{v} / c^{2}\right) .
$$

The inverse transformations are

$$
\bar{E} \rightarrow E=\gamma\left(\left[m c^{2}+\bar{E}\right]+\overline{\boldsymbol{p}} \cdot \boldsymbol{v}\right)-m c^{2},
$$

and

$$
\overline{\boldsymbol{p}} \rightarrow \boldsymbol{p}=\gamma\left(\left[m c^{2}+\bar{E}\right] \boldsymbol{v} / c^{2}+\overline{\boldsymbol{p}}\right) .
$$

These affine transformations make perfectly good physical sense once you realise that, with the conventions of this subsection, the $E$ 's in question are just the relativistic kinetic energies - quantities that are normally denoted by $K$ :

$$
E_{\text {here }}=E_{\text {total }}-m c^{2}=K \text {. }
$$

Then

$$
K \rightarrow \bar{K}=\gamma\left(\left[m c^{2}+K\right]-\boldsymbol{p} \cdot \boldsymbol{v}\right)-m c^{2},
$$

and

$$
\boldsymbol{p} \rightarrow \overline{\boldsymbol{p}}=\gamma\left(\boldsymbol{p}-\left[m c^{2}+K\right] \boldsymbol{v} / c^{2}\right),
$$

while

$$
\bar{K} \rightarrow K=\gamma\left(\left[m c^{2}+\bar{K}\right]+\overline{\boldsymbol{p}} \cdot \boldsymbol{v}\right)-m c^{2},
$$

and

$$
\overline{\boldsymbol{p}} \rightarrow \boldsymbol{p}=\gamma\left(\left[m c^{2}+\bar{K}\right] \boldsymbol{v} / c^{2}+\overline{\boldsymbol{p}}\right) .
$$

These are manifestly just a disguised form of the usual Lorentz transformations. Note that the formal $c \rightarrow \infty$ limit of these (slightly nonstandard) affine equations is

$$
K \rightarrow \bar{K}=K-\boldsymbol{p} \cdot \boldsymbol{v}+\frac{1}{2} m\|\boldsymbol{v}\|^{2} ; \quad \boldsymbol{p} \rightarrow \overline{\boldsymbol{p}}=\boldsymbol{p}-m \boldsymbol{v} ;
$$

and

$$
\bar{K} \rightarrow K=\bar{K}+\overline{\boldsymbol{p}} \cdot \boldsymbol{v}+\frac{1}{2} m\|\boldsymbol{v}\|^{2} ; \quad \overline{\boldsymbol{p}} \rightarrow \boldsymbol{p}=\overline{\boldsymbol{p}}+m \boldsymbol{v} .
$$

These are the (affine) transformation laws for (the usual form of) the Galileo group.

Again, the somewhat subtle message to take from this is that since the affine parameters $\epsilon$ and $\boldsymbol{\pi}$, and $\bar{\epsilon}$ and $\overline{\boldsymbol{\pi}}$, are already so important in situations of extremely high symmetry (the Lorentz group, the Galileo group), then they are also likely to be important in any situations where these symmetries are broken. 


\subsubsection{Galileo group (linear version)}

The previous discussion suggests that there might be some (perhaps nonstandard) set of conventions that would make the energy and momentum transform linearly for the Galileo group. That is, there might be some way of arranging things so that for the Galileo group

$$
E \rightarrow \bar{E}=E-\boldsymbol{p} \cdot \boldsymbol{v} ; \quad \boldsymbol{p} \rightarrow \overline{\boldsymbol{p}}=\boldsymbol{p} .
$$

How would we do that? It will have to be something rather unusual. Choose the following Lagrangians:

$$
L=\frac{1}{2} m\|\dot{\mathbf{x}}\|^{2} ; \quad \bar{L}=\frac{1}{2} m\|\dot{\overline{\mathbf{x}}}+\boldsymbol{v}\|^{2} .
$$

Then the momenta are

$$
\boldsymbol{p}=m \dot{\mathbf{x}} ; \quad \overline{\boldsymbol{p}}=m(\dot{\overline{\mathbf{x}}}+\boldsymbol{v})=m \dot{\mathbf{x}}=\boldsymbol{p} .
$$

The energy in the aether frame is (as usual)

$$
E=\boldsymbol{p} \cdot \dot{\mathbf{x}}-L=\frac{1}{2} m\|\dot{\mathbf{x}}\|^{2}
$$

However with these conventions the energy in the moving frame is

$$
\begin{aligned}
\bar{E}=\overline{\boldsymbol{p}} \cdot \dot{\overline{\mathbf{x}}}-\bar{L} & =m(\dot{\overline{\mathbf{x}}}+\boldsymbol{v}) \cdot \dot{\overline{\mathbf{x}}}-\frac{1}{2} m\|\dot{\overline{\mathbf{x}}}+\boldsymbol{v}\|^{2} \\
& =m \dot{\mathbf{x}} \cdot(\dot{\mathbf{x}}-\boldsymbol{v})-\frac{1}{2} m\|\dot{\mathbf{x}}\|^{2} \\
& =\frac{1}{2} m\|\dot{\mathbf{x}}\|^{2}-m \dot{\mathbf{x}} \cdot \boldsymbol{v}=E-\boldsymbol{p} \cdot \boldsymbol{v} .
\end{aligned}
$$

Now $\bar{L}=\frac{1}{2} m\|\dot{\overline{\mathbf{x}}}+\boldsymbol{v}\|^{2}$, is certainly an "odd" and "unusual" Lagrangian to choose for a free non-relativistic particle in the moving frame, but it is by no means "wrong" it certainly does the job. One certainly has the correct equations of motion $\ddot{\overline{\mathbf{x}}}=0$, and for this definition of energy and momentum, albeit "odd" and "unusual", the energy-momentum transformation laws are explicitly linear:

$$
\bar{p}=\boldsymbol{p} ; \quad \bar{E}=E-\boldsymbol{p} \cdot \boldsymbol{v} .
$$

Note that we have made the quantities $\{\epsilon, \boldsymbol{\pi}\}$ and $\{\bar{\epsilon}, \overline{\boldsymbol{\pi}}\}$ simple, in fact zero, at the price of making the moving-frame Lagrangian complicated. (For some comments in a similar vein, see section II.A of reference [219].) 


\subsubsection{Considerations regarding affine versus linear}

When looking at how this general framework and formalism applies to the Lorentz group we saw that there were good choices for $\epsilon$ and $\boldsymbol{\pi}$, and $\bar{\epsilon}$ and $\overline{\boldsymbol{\pi}}$, and also "bad" (or rather, sub-optimal) choices. There seems to be considerable freedom in how one picks $\epsilon$ and $\boldsymbol{\pi}$, and $\bar{\epsilon}$ and $\overline{\boldsymbol{\pi}}$, and so considerable freedom in choosing affine versus linear transformations for the 4-momentum. Can this freedom be used to improve things? If one is working in a region of parameter space that is "close" to special relativity (a "perturbative" deviation from special relativity) then linear transformations for the 4-momentum would seem to be the most appropriate choice. If one is working in a region of parameter space that is "close" to Galillean relativity (a "perturbative" deviation from Newtonian mechanics) then affine transformations for the 4-momentum would seem to be the most appropriate choice. The general situation is somewhat unclear, but it seems advisable to retain the generality of the full affine transformations as long as possible.

\subsection{On-shell relations for energy and momentum}

In any particular inertial frame if one measures the energy $E$ and momentum $\boldsymbol{p}$ of an on-shell particle then there will be some relation between them; an on-shell energy-momentum relation $E=E(\boldsymbol{p})$. One normally expects a very tight connection between the functional form of these energy-momentum relations and the functional form of the transformations between inertial frames - unfortunately this very tight connection is intimately related with adopting the relativity principle, and will in general fail once the relativity principle is abandoned. That is, in Lorentz-violating theories the functional form of the energy-momentum relations and the functional form of the transformations between inertial frames can be (and often are) independent of each other.

For this reason, one important matter to address is to understand what happens to quantities that are considered as intrinsic properties of a particle in a special relativistic scenario, such as the rest-mass, when the relativity principle is dropped.

\subsubsection{Rest energy without the relativity principle}

To see how this comes about, consider the preferred (aether) frame $F$, and in that frame suppose you measure the energy $E$ and momentum $\boldsymbol{p}$ of the same particle in a number of different kinematic states to map out the energy-momentum relation $E=E(\boldsymbol{p})$ in the aether frame. To each momentum $\boldsymbol{p}$ we associate a 3 -velocity 
$\boldsymbol{v}=\partial E / \partial \boldsymbol{p}$. Now go to the rest frame $\bar{F}$ of the particle (of course the rest frame of the particle is moving with respect to the aether). In the rest frame the particle will by definition have 3 -velocity zero $\overline{\boldsymbol{v}}=0$, and will have some energy, call it the rest-energy $\bar{E}=E_{0}$ and some momentum, call it the rest-momentum $\overline{\boldsymbol{p}}=\boldsymbol{p}_{0}$.

If the relativity principle holds then the rest-energy and rest-momentum must be intrinsic properties of the particle that cannot depend on its velocity with respect to the aether - and in particular the rest-momentum is most typically chosen to be zero. But once one has preferred frame effects the rest-energy and rest-momentum can very definitely depend on the state of motion with respect to the aether. That is, generally we will have $\bar{E}=E_{0}(\bar{F})$ and $\overline{\boldsymbol{p}}=\boldsymbol{p}_{0}(\bar{F})$.

Transforming back to the aether frame we now see

$$
E=\gamma E_{0}(\bar{F})+\boldsymbol{p}_{0}(\bar{F})^{T} \Sigma \boldsymbol{v}+\gamma \bar{\epsilon}+\overline{\boldsymbol{\pi}}^{T} \Sigma \boldsymbol{v}-\epsilon
$$

and

$$
\boldsymbol{p}=\gamma E_{0}(\bar{F}) \boldsymbol{u}+\Sigma^{T} \boldsymbol{p}_{0}(\bar{F})+\gamma \bar{\epsilon} \boldsymbol{u}+\Sigma^{T} \overline{\boldsymbol{\pi}}-\boldsymbol{\pi} .
$$

In general, unless further assumptions are made, this is the best one can do.

We now use the freedom to choose the quantities $\{\epsilon, \boldsymbol{\pi}\}$ and $\{\bar{\epsilon}, \overline{\boldsymbol{\pi}}\}$ to make life as simple as possible. Without any real loss of generality we can choose $\overline{\boldsymbol{\pi}}=-\boldsymbol{p}_{0}(\bar{F})$ in which case

$$
E=\gamma E_{0}(\bar{F})+\gamma \bar{\epsilon}-\epsilon
$$

and

$$
\boldsymbol{p}=\gamma E_{0}(\bar{F}) \boldsymbol{u}+\gamma \bar{\epsilon} \boldsymbol{u}-\boldsymbol{\pi} .
$$

(This is equivalent to choosing conventions so that in the rest frame the total "effective" rest momentum $\boldsymbol{p}_{0}+\overline{\boldsymbol{\pi}}=\mathbf{0}$.) Let us now for definiteness choose $\epsilon=0$ and $\boldsymbol{\pi}=\mathbf{0}$, then

$$
E=\gamma\left[E_{0}(\bar{F})+\bar{\epsilon}\right]
$$

and

$$
\boldsymbol{p}=\gamma\left[E_{0}(\bar{F})+\bar{\epsilon}\right] \boldsymbol{u}=E \boldsymbol{u} .
$$

(We have done things in this manner so that it becomes clear just how general the relation $\boldsymbol{p}=E \boldsymbol{u}$ really is.) Finally choose $\bar{\epsilon}=0$, then with these choices we can write

$$
E=\gamma E_{0}(\bar{F})
$$

and

$$
\boldsymbol{p}=\gamma E_{0}(\bar{F}) \boldsymbol{u}=E \boldsymbol{u}
$$


Introduce an arbitrary but fixed constant $c$ with the dimensions of velocity (not necessarily the speed of light), and some arbitrary function $\varpi(\bar{F})$ which is completely at our disposal. Then in the aether frame we can write

$$
E^{2}-\varpi\|\boldsymbol{p}\|^{2} c^{2}=\gamma^{2}\left(1-\varpi c^{2}\|\boldsymbol{u}\|^{2}\right) E_{0}^{2}(\bar{F}) .
$$

Two particularly useful (but by no means inevitable) choices are to take:

- Choose $\varpi \rightarrow 1$ so that

$$
E^{2}-\|\boldsymbol{p}\|^{2} c^{2}=\gamma^{2}\left(1-c^{2}\|\boldsymbol{u}\|^{2}\right) E_{0}^{2}(\bar{F}) .
$$

- Choose $\varpi \rightarrow\|\boldsymbol{v}\|^{2} /\left(\|\boldsymbol{u}\|^{2} c^{4}\right)$ so that

$$
E^{2}-\frac{\|\boldsymbol{v}\|^{2}}{\|\boldsymbol{u}\|^{2} c^{2}}\|\boldsymbol{p}\|^{2}=\gamma^{2}\left(1-v^{2} / c^{2}\right) E_{0}^{2}(\bar{F})
$$

In the case of exact Lorentz invariance we most usefully choose $\varpi \rightarrow 1$, with the constant $c$ being interpreted as the speed of light, and $\gamma \rightarrow 1 / \sqrt{1-v^{2} / c^{2}}$, while $\boldsymbol{u}=\boldsymbol{v} / c^{2}$. Furthermore $E_{0}$ is then independent of $\boldsymbol{v}$, so in this case one recovers the usual kinematic relation $E^{2}-\|\boldsymbol{p}\|^{2} c^{2}=E_{0}^{2}$, while (as expected) $E=\gamma E_{0}$ and $\boldsymbol{p}=\gamma E_{0} \boldsymbol{v} / c^{2}=E \boldsymbol{v} / c^{2}$. In the absence of Lorentz invariance one generically has to live with the more complicated kinematics presented above. The notion of rest energy $E_{0}$ still makes perfectly good sense, but the rest energy can depend on the particle's state of motion through the aether, $E_{0}(\bar{F})$, and the relation to 4-momentum is considerably more subtle than one might have expected.

The key point here is that the energy-momentum relation $E(\boldsymbol{p})$ and the transformation matrix $M$ are in general independent of each other; knowing one does not necessarily give you the other (except when Lorentz invariance is assumed, or some similar restriction is imposed). There are two additional special cases of considerable interest, which we now discuss.

\subsubsection{Invariant rest energy without the relativity principle}

One can speculate or hypothesise that for unknown reasons the internal structure of elementary and composite particles self-regulates so that rest energies are independent of one's state of motion through the aether. One still has rather unusual behaviour in that

$$
E=\gamma E_{0} ; \quad \boldsymbol{p}=\gamma E_{0} \boldsymbol{u}=E \boldsymbol{u}
$$

while

$$
E^{2}-\varpi\|\boldsymbol{p}\|^{2} c^{2}=\gamma^{2}\left(1-\varpi c^{2}\|\boldsymbol{u}\|^{2}\right) E_{0}^{2}
$$


(Remember $\boldsymbol{u}$ is not necessarily parallel to $\boldsymbol{v}$, neither does $\|\boldsymbol{u}\|$ equal $\|\boldsymbol{v}\|$, they do not even have the same dimensions. In addition, all three of the functions $\varpi(\bar{F})$, $\gamma(\bar{F})$, and $\boldsymbol{u}(\bar{F})$ can depend on the particle's state of motion with respect to the aether. In fact $\varpi$ is entirely arbitrary and can be adjusted to taste - we will have cause to use this freedom below.) So even with an invariant rest mass (and this is a rather strong assumption) the 4-momentum of a moving particle is rather definitely non-trivial.

Starting from this assumption, we shall now proceed to introduce the first of the two minimally Lorentz-violating models.

\subsubsection{First minimalist Lorentz-violating model}

Another important special case to consider is to assume that the transformations between inertial frames are the usual Lorentz transformations but the energy-momentum relation - for at least some of the particles — is not Lorentz invariant, see figure 4.2. This is less bizarre than one might at first glance suspect. The point is that the physical clocks and rulers we use in our laboratories satisfy the Lorentz invariant physics rather accurately - and we have good phenomenological/observational evidence that (apart possibly from the neutrino sector) Lorentz invariance is an extremely good approximation to empirical reality. So it makes good sense to work in an approximation where all physical clocks and rulers are exactly Lorentz invariant, and the only Lorentz violations are hiding in some specific particle sector (e.g. the neutrinos). In contrast, if there are significant Lorentz violations in the physics underpinning one's clocks and rulers, then using the Lorentz transformations to inter-relate the space and time coordinates determined by those clocks and rulers would be a very bad and physically unjustified approximation.

More generally let us consider a model where Lorentz-violating physics is confined to a specific sub-sector of the particle physics spectrum. In this situation the rest energy of the Lorentz-violating particles can still depend on their state of motion with respect to the aether. In the aether frame we then have

$$
E=\gamma E_{0}(\bar{F}) ; \quad \boldsymbol{p}=\gamma E_{0}(\bar{F}) \boldsymbol{v} / c^{2}=E \boldsymbol{v} / c^{2}
$$

while

$$
E^{2}-\|\boldsymbol{p}\|^{2} c^{2}=E_{0}^{2}(\bar{F})
$$

Again, even in this simplified situation, the 4-momentum and the kinematic relation are rather definitely non-trivial. If we now transform to a third inertial frame $\overline{\bar{F}}$, 


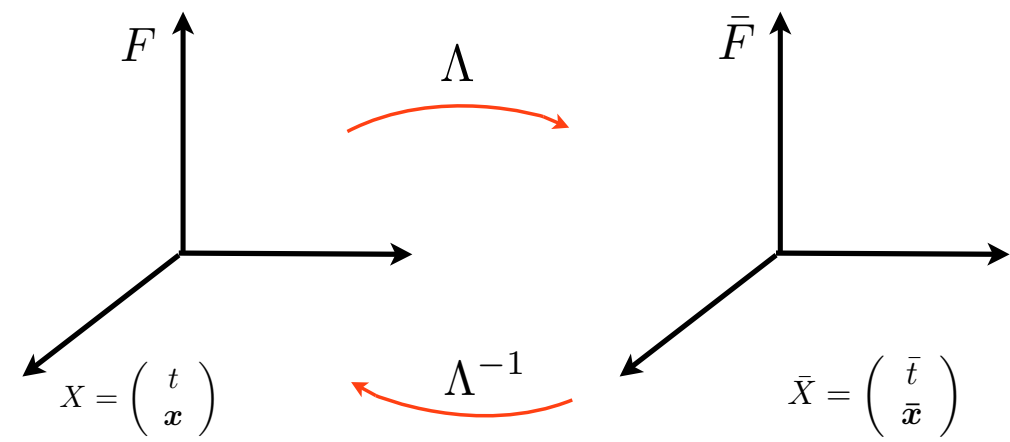

Figure 4.2: In this first Lorentz-violating model the transformations between two inertial frames $F$ and $\bar{F}$ are the usual Lorentz transformations $\Lambda$ in which we assume that the physics of clock and rulers fulfils Lorentz symmetry. The Lorentz-violating sector is the energy-momentum relation of some particle species that does not contribute significantly to the physics of clocks and rulers.

then certainly

$$
\overline{\bar{E}}^{2}-\|\overline{\overline{\boldsymbol{p}}}\|^{2} c^{2}=E^{2}-\|\boldsymbol{p}\|^{2} c^{2}=E_{0}^{2}(\bar{F})
$$

is a Lorentz invariant, but the specific value of this Lorentz invariant quantity depends on the absolute state of motion of the Lorentz-violating particle as viewed from the aether frame. The way we have currently set things up, this rest energy could even be direction dependent - no isotropy assumption (at least in the Lorentzviolating sector) has yet been made. If we now add the additional assumption that the Lorentz-violating physics is isotropic in the aether frame then

$$
E=\gamma E_{0}(v) ; \quad \boldsymbol{p}=\gamma E_{0}(v) \boldsymbol{v} / c^{2}=E \boldsymbol{v} / c^{2} ;
$$

while

$$
E^{2}-\|\boldsymbol{p}\|^{2} c^{2}=E_{0}^{2}(v) .
$$

So we rather explicitly see the manner in which absolute speed with respect to the aether would formally affect on-shell particle energy-momentum relations. We emphasise that in this model, even though the Lorentz-violating particles do not have a Lorentz invariant energy-momentum relation, their energies and 3-momenta nevertheless transform in the usual manner under Lorentz transformations. To make this look more relativistic, one could introduce a 4 -velocity $V_{\text {aether }}$ for the aether, 
and another 4-velocity $V_{\text {particle }}$ for the Lorentz-violating particle. The speed $v$ of the Lorentz violating particle with respect to the aether is then the usual explicit function of the 4 -inner-product $\eta\left(V_{\text {aether }}, V_{\text {particle }}\right)$ and the kinematic relation takes the form

$$
E^{2}-\|\boldsymbol{p}\|^{2} c^{2}=E_{0}^{2}\left(\eta\left(V_{\text {aether }}, V_{\text {particle }}\right)\right) .
$$

This model is the first of the "minimalist" models of Lorentz violation we refer to in the introduction.

Broadly speaking, something along the lines of this minimalist model is often implicitly adopted in currently extant analyses of Lorentz violating models, but often without the relevant assumptions being clearly and explicitly laid out. Many current analyses implicitly treat Lorentz violation perturbatively, modelling reality by a Lorentz invariant "core" subject to Lorentz-violating "perturbations". (This is true for instance in the Kostelecky et al. Standard Model Extension [59, 91, 180,186, the Coleman-Glashow analyses [88, 89], see also [87, and the Jacobson-LiberatiMattingly analyses [158, 165, 198, 210, 211], see also [205].) Typically, to a first approximation one ignores the effect of any (presumably small) Lorentz-violating physics on the internal structure one's clocks and rulers, thereby implicitly justifying the use of ordinary Lorentz transformations for one's experimentally measured energy and momenta, and focuses attention on subtle deviations from Lorentz invariance that might be probed by suitably designed null experiments. However it should be very much emphasised that if the effect of Lorentz-violating physics ever has non-perturbatively large effects on the internal structure one's clocks and rulers, then one can no longer safely use the ordinary Lorentz transformations for experimentally determined energy and momenta - and instead of adopting some version of the minimalist model above one must then resort to the full power of the preceding analysis.

\subsubsection{Considerations regarding on-shell energy-momentum}

We emphasise that we have gone to all this trouble in setting up a very general formalism in order to have a coherent and consistent framework to operate in once we begin to entertain possible departures from Lorentz invariance. Many of the results derived so far are quite unexpected when one has been trained to always think in a Lorentz invariant (or even Galilean invariant) and relativity principle respecting manner. 


\subsection{Adding more constraints}

Let us now see what extra conditions we would have to add to obtain standard physics.

\subsubsection{Linearity plus isotropy}

Now let us add the assumption of isotropy - specifically that physics is isotropic in the preferred frame, the aether frame. In particular this means that in the inertial transformation matrices

$$
\begin{gathered}
M=\left[\begin{array}{c|c}
\gamma & -\gamma \boldsymbol{u}^{T} \\
\hline-\Sigma \boldsymbol{v} & \Sigma
\end{array}\right] \\
M^{-1}=\left[\begin{array}{c|c}
\gamma^{-1}(1-\boldsymbol{u} \cdot \boldsymbol{v})^{-1} & \boldsymbol{u}^{T}(I-\boldsymbol{v} \otimes \boldsymbol{u})^{-1} \Sigma^{-1} \\
\hline \gamma^{-1}(1-\boldsymbol{u} \cdot \boldsymbol{v})^{-1} \boldsymbol{v} & (I-\boldsymbol{v} \otimes \boldsymbol{u})^{-1} \Sigma^{-1}
\end{array}\right]
\end{gathered}
$$

all vectors and matrices should be constructible only using the vector $\boldsymbol{v}$ and its magnitude - there are now assumed to be no preferred principal axes for the universe. We are also assuming that the frames $F$ and $\bar{F}$ are "aligned" (not rotated with respect to each other). Then isotropy amounts to

$$
\boldsymbol{u} \| \boldsymbol{v} ; \quad \Sigma=a I+b \boldsymbol{v} \otimes \boldsymbol{v} .
$$

In fact it is now useful to introduce an arbitrary but fixed unspecified constant $c$ with the dimensions of velocity, and a dimensionless parameter $\zeta$, to write

$$
\boldsymbol{u}=\zeta \boldsymbol{v} / c^{2} .
$$

Similarly, let us introduce dimensionless variables $\chi$ and $\xi$ to write

$$
\Sigma=\gamma \chi \mathbf{n} \otimes \mathbf{n}+\xi[I-\mathbf{n} \otimes \mathbf{n}]
$$

Recall $\boldsymbol{v}=v \mathbf{n}$. By appealing to isotropy, the four quantities $\gamma, \chi, \zeta$, and $\xi$ are arbitrary dimensionless functions of the dimensionless variable $v^{2} / c^{2}$. By combining linearity with isotropy in this manner we have obtained a variant of the RobertsonMansouri-Sexl framework; see [206, 239], and section 3.2 of [210]. (The RMS formalism invokes several other technical assumptions not relevant to the current discussion, and is not quite identical to our own framework.) Note that the quantities $\gamma, \chi, \zeta$, and $\xi$ can still depend on the internal structure of one's clocks and rulers.

We now have

$$
M=\left[\begin{array}{c|c}
\gamma & -\gamma \zeta \boldsymbol{v}^{T} / c^{2} \\
\hline-\gamma \chi \boldsymbol{v} & \gamma \chi \mathbf{n} \otimes \mathbf{n}+\xi[I-\mathbf{n} \otimes \mathbf{n}]
\end{array}\right] .
$$


An intermediate step in calculating the inverse transformation is

$$
M^{-1}=\left[\begin{array}{c|c}
\gamma^{-1}\left(1-\zeta v^{2} / c^{2}\right)^{-1} & \zeta \boldsymbol{v}^{T}\left(I-\zeta \boldsymbol{v} \boldsymbol{v}^{T} / c^{2}\right)^{-1} \Sigma^{-1} / c^{2} \\
\hline \gamma^{-1}\left(1-\zeta v^{2} / c^{2}\right)^{-1} \boldsymbol{v} & \left(I-\zeta \boldsymbol{v} \boldsymbol{v}^{T} / c^{2}\right)^{-1} \Sigma^{-1}
\end{array}\right]
$$

But

$$
\begin{aligned}
\Sigma\left(I-\zeta \boldsymbol{v} \boldsymbol{v}^{T} / c^{2}\right) & =(\gamma \chi \mathbf{n} \otimes \mathbf{n}+\xi[I-\mathbf{n} \otimes \mathbf{n}])\left(I-\left[\zeta v^{2} / c^{2}\right] \mathbf{n} \otimes \mathbf{n}\right) \\
& =\gamma \chi\left[1-\zeta v^{2} / c^{2}\right] \mathbf{n} \otimes \mathbf{n}+\xi[I-\mathbf{n} \otimes \mathbf{n}],
\end{aligned}
$$

whence

$$
\left(I-\zeta \boldsymbol{v} \boldsymbol{v}^{T} / c^{2}\right)^{-1} \Sigma^{-1}=\gamma^{-1} \chi^{-1}\left[1-\zeta v^{2} / c^{2}\right]^{-1} \mathbf{n} \otimes \mathbf{n}+\xi^{-1}[I-\mathbf{n} \otimes \mathbf{n}] .
$$

So the inverse transformation matrix simplifies to

$$
M^{-1}=\left[\begin{array}{c|c}
\gamma^{-1}\left(1-\zeta v^{2} / c^{2}\right)^{-1} & \gamma^{-1}\left(1-\zeta v^{2} / c^{2}\right)^{-1} \zeta \chi^{-1} \boldsymbol{v} v^{T} / c^{2} \\
\hline \gamma^{-1}\left(1-\zeta v^{2} / c^{2}\right)^{-1} \boldsymbol{v} & \gamma^{-1} \chi^{-1}\left[1-\zeta v^{2} / c^{2}\right]^{-1} \mathbf{n} \otimes \mathbf{n}+\xi^{-1}[I-\mathbf{n} \otimes \mathbf{n}]
\end{array}\right] .
$$

By a specialization of our previous discussion:

- The velocity of the moving frame with respect to the aether is $\boldsymbol{v}$.

- The velocity of the aether with respect to the moving frame is $-\chi \boldsymbol{v}$.

- These are now at least collinear, and in fact anti-parallel, but can still differ in magnitude; they are still not equal-but-opposite.

If we rotate to align $\boldsymbol{v}$ along the $\hat{\mathbf{x}}$ axis this looks a little simpler:

$$
M=\left[\begin{array}{c|c|c}
\gamma & -\gamma \zeta v / c^{2} & \mathbf{0}^{T} \\
\hline-\gamma \chi v & \gamma \chi & \mathbf{0}^{T} \\
\hline \mathbf{0} & \mathbf{0} & \xi I
\end{array}\right]
$$

and

$$
M^{-1}=\left[\begin{array}{c|c|c}
\gamma^{-1}\left(1-\zeta v^{2} / c^{2}\right)^{-1} & \gamma^{-1}\left(1-\zeta v^{2} / c^{2}\right)^{-1} \zeta \chi^{-1} v / c^{2} & \mathbf{0}^{T} \\
\hline \gamma^{-1}\left(1-\zeta v^{2} / c^{2}\right)^{-1} v & \gamma^{-1} \chi^{-1}\left[1-\zeta v^{2} / c^{2}\right]^{-1} & \mathbf{0}^{T} \\
\hline \mathbf{0} & \mathbf{0} & \xi^{-1} I
\end{array}\right] .
$$

This is as far as you can get with linearity plus isotropy - you still have four arbitrary functions $\gamma\left(v^{2} / c^{2}\right), \chi\left(v^{2} / c^{2}\right), \zeta\left(v^{2} / c^{2}\right)$, and $\xi\left(v^{2} / c^{2}\right)$ to deal with, but at 
least it is no longer an arbitrary $4 \times 4$ matrix with 16 free components. The set of transformations is still not a group, just a groupoid/pseudogroup.

In view of the isotropy assumption particle rest masses $E_{0}$ should depend only on the speed with respect to the aether, hence be of the form $E_{0}(v)$. Specializing our earlier discussion, with $\varpi, \gamma$, and $\zeta$ being velocity dependent, in the aether frame we would have

$$
E=\gamma E_{0}(v) ; \quad \boldsymbol{p}=\gamma \zeta E_{0}(v) \boldsymbol{v} / c^{2}
$$

with

$$
E^{2}-\varpi\|\boldsymbol{p}\|^{2} c^{2}=\gamma^{2}\left[1-\varpi \zeta^{2} v^{2} / c^{2}\right] E_{0}(v)^{2} .
$$

Note that Lorentz invariance corresponds to setting $\chi=\zeta=\xi=1$, with $\gamma=$ $1 / \sqrt{1-v^{2} / c^{2}}$, (and in addition demanding $\varpi \rightarrow 1$ and that $E_{0}$ be constant).

The Galilean limit is somewhat delicate: Physically we want to be looking at some sort of low velocity limit. When moving at zero velocity through the aether we expect $M \rightarrow I$ (corresponding to the trivial transformation), so we must have $\chi(0)=$ $\gamma(0)=\xi(0)=1$. In contrast $\zeta(0)$ should be finite but is otherwise unconstrained. However $c$ is at this stage just some constant with the dimensions of velocity, it does not yet have any deeper physical interpretation, so one can simply absorb $\zeta(0)$ into a redefinition of $c$ and so effectively set $\zeta(0) \rightarrow 1$.

- In the transformation matrices $M$ and $M^{-1}$, this low-velocity limit corresponds to $\zeta=\chi=\gamma=\xi=1$, with $\|\boldsymbol{v}\| \ll c$.

- Because of isotropy, in the low-velocity limit we must have both

$$
\gamma(v) \approx 1+\frac{1}{2} \gamma_{2} v^{2} / c^{2}+\ldots, \quad \text { and } \quad \zeta(v) \approx 1+\frac{1}{2} \zeta_{2} v^{2} / c^{2}+\ldots
$$

Furthermore

$$
E_{0}(v)=E_{0}(0)\left\{1+\frac{1}{2} \kappa_{2} v^{2} / c^{2}+\ldots\right\}
$$

so that:

$$
E \approx E_{0}(0)+\frac{1}{2}\left[E_{0}(0) / c^{2}\right]\left\{\gamma_{2}+\kappa_{2}\right\} v^{2}+\ldots ; \quad \boldsymbol{p} \approx\left[E_{0}(0) / c^{2}\right] \boldsymbol{v}+\ldots
$$

- If we define the low-velocity effective mass by $m_{\mathrm{eff}}=E_{0}(0) / c^{2}$ then

$$
E \approx m_{\mathrm{eff}} c^{2}+\left\{\gamma_{2}+\kappa_{2}\right\} \frac{\|\boldsymbol{p}\|^{2}}{2 m_{\mathrm{eff}}}+\ldots ; \quad \boldsymbol{p} \approx m_{\mathrm{eff}} \boldsymbol{v}+\ldots
$$

So there is a sensible low-velocity limit, though it is perhaps more subtle than one might have thought. 


\subsubsection{Linearity plus isotropy plus reciprocity}

It is sometimes useful to restrict attention to situations where $M^{-1}(\boldsymbol{v})=M(-\boldsymbol{v})$. Note that this is an additional axiom beyond homogeneity and isotropy.

- This is (one version of) the so-called reciprocity principle. It is still weaker than the relativity principle.

- This version of the reciprocity principle, because it also makes assumptions about the transverse directions, is very slightly stronger than asserting that the velocity of any inertial frame as seen from the aether is minus the velocity of the aether as seen from that inertial frame [67].

- The way we have formulated it, reciprocity implies both $\chi=1$ and $\xi=1$, and in addition imposes the constraint

$$
\gamma=\frac{1}{\sqrt{1-\zeta v^{2} / c^{2}}}
$$

To see this, compare $M$ with $M^{-1}$ above, and note that $M^{-1}(\boldsymbol{v})=M(-\boldsymbol{v})$ implies the three relations:

$$
\begin{gathered}
\gamma=\gamma^{-1}\left(1-\zeta v^{2} / c^{2}\right)^{-1} \\
\gamma \chi=\gamma^{-1} \chi^{-1}\left(1-\zeta v^{2} / c^{2}\right)^{-1} \\
\xi=\xi^{-1}
\end{gathered}
$$

Solving, we see

$$
\xi=1 ; \quad \chi=1 ; \quad \gamma^{2}\left(1-\zeta v^{2} / c^{2}\right)=1 .
$$

Then

$$
\begin{gathered}
M=\left[\begin{array}{c|c|c}
\gamma & -\gamma \zeta v / c^{2} & \mathbf{0}^{T} \\
\hline-\gamma v & \gamma & \mathbf{0}^{T} \\
\hline \mathbf{0} & \mathbf{0} & I
\end{array}\right] ; \\
M^{-1}=\left[\begin{array}{c|c|c}
\gamma & \gamma \zeta v / c^{2} & \mathbf{0}^{T} \\
\hline \gamma v & \gamma & \mathbf{0}^{T} \\
\hline \mathbf{0} & \mathbf{0} & I
\end{array}\right] ;
\end{gathered}
$$

subject to the constraint

$$
\gamma=\frac{1}{\sqrt{1-\zeta v^{2} / c^{2}}}
$$

Note that you now only have one free function $\zeta\left(v^{2} / c^{2}\right)$, everything else is determined. 
- Working along a somewhat different route, it has been shown [67] that combining relativity+homogeneity+isotropy implies (at least one version of) the reciprocity principle.

- Note that adopting the principle of reciprocity implies the set $\{M(\boldsymbol{v})\}$ is now closed under matrix inversion, though it is still not a group.

- This is not quite special relativity [or even Galilean relativity], but it is getting awfully close.

\subsubsection{Second minimalist Lorentz-violating model}

Since the model above (linearity plus isotropy plus reciprocity) is a simple onefunction violation of special relativity, it holds a special place in the set of all Lorentz violating (relativity principle violating) theories - this is arguably the simplest violation of special relativity one can have at the level of the transformations between inertial frames. At the level of the coordinate transformations

$$
\begin{gathered}
t \rightarrow \bar{t}=\frac{t-\zeta(v) v x / c^{2}}{\sqrt{1-\zeta(v) v^{2} / c^{2}}} \\
x \rightarrow \bar{x}=\frac{x-v t}{\sqrt{1-\zeta(v) v^{2} / c^{2}}} \\
y \rightarrow \bar{y}=y ; \quad z \rightarrow \bar{z}=z .
\end{gathered}
$$

The closest one can get to a notion of "interval" is to observe

$$
\frac{c^{2}(\Delta t)^{2}}{\zeta(v)}-\|\Delta \mathbf{x}\|^{2}=\frac{c^{2}(\Delta \bar{t})^{2}}{\zeta(v)}-\|\Delta \overline{\mathbf{x}}\|^{2}
$$

Recall that $\zeta(v)$ depends on the absolute speed of the moving frame through the aether, so this is only a 2 -frame invariant, it is not a general invariant for arbitrary combinations of inertial frames. To be explicit about this, let $F_{1}$ and $F_{2}$ be two moving frames, then

$$
\frac{c^{2}(\Delta t)^{2}}{\zeta\left(v_{1}\right)}-\|\Delta \mathbf{x}\|^{2}=\frac{c^{2}\left(\Delta t_{1}\right)^{2}}{\zeta\left(v_{1}\right)}-\left\|\Delta \mathbf{x}_{1}\right\|^{2},
$$

and

$$
\frac{c^{2}(\Delta t)^{2}}{\zeta\left(v_{2}\right)}-\|\Delta \mathbf{x}\|^{2}=\frac{c^{2}\left(\Delta t_{2}\right)^{2}}{\zeta\left(v_{2}\right)}-\left\|\Delta \mathbf{x}_{2}\right\|^{2} .
$$

But (ultimately due to the lack of the relativity principle, and the consequent lack of group structure for the transformations) there is, under the current assumptions, 


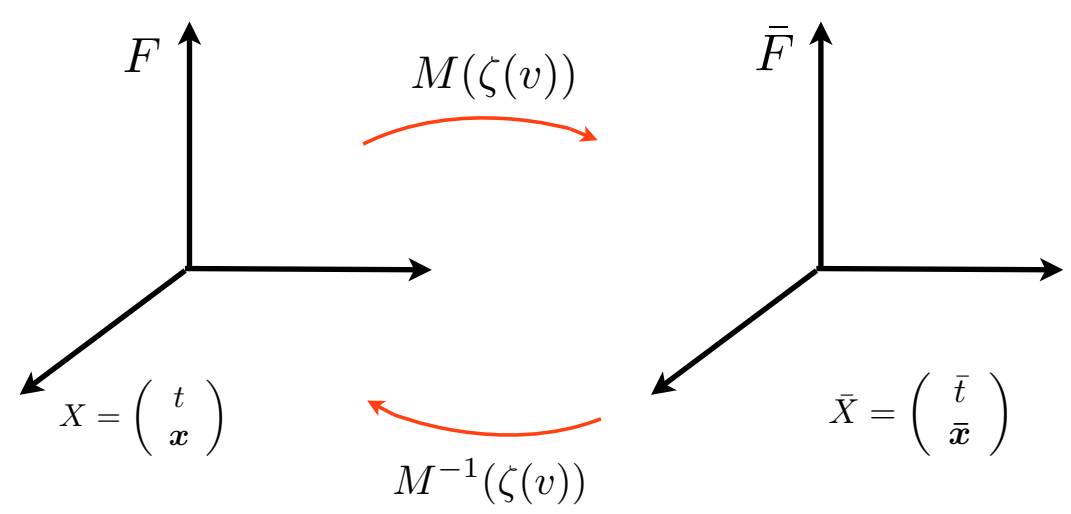

Figure 4.3: In this second Lorentz-violating model the transformations $M$ and $M^{-1}$ between the aether frame $F$ and another inertial frame $\bar{F}$ depends only on one parameter, the absolute speed $v$ of the moving frame through the aether. Due to the lack of the relativity principle, when considering on-shell particle energy-momentum relations, the invariant masses $E_{0}(v)$ can depend on absolute velocity with respect to the aether.

no simple relationship of this type connecting the measurements in inertial frame $F_{1}$ with those in inertial frame $F_{2}$, see figure 4.3 .

When we turn to on-shell particle energy-momentum relations we still have invariant masses $E_{0}(v)$ that can depend on absolute velocity with respect to the aether. Therefore, in view of our previous discussion, in the aether frame we would have

$$
E=\frac{E_{0}(v)}{\sqrt{1-\zeta(v) v^{2} / c^{2}}} ; \quad \boldsymbol{p}=\frac{\zeta(v) E_{0}(v) \boldsymbol{v} / c^{2}}{\sqrt{1-\zeta(v) v^{2} / c^{2}}}=E \boldsymbol{v} / c^{2}
$$

with

$$
E^{2}-\varpi(v)\|\boldsymbol{p}\|^{2} c^{2}=\left[\frac{1-\varpi(v) \zeta^{2}(v) v^{2} / c^{2}}{1-\zeta(v) v^{2} / c^{2}}\right] E_{0}(v)^{2} .
$$

But $\varpi(v)$ is a completely arbitrary function that is entirely at our disposal, so in the current context it makes sense to choose $\varpi=1 / \zeta$ in which case

$$
E^{2}-\zeta^{-1}(v)\|\boldsymbol{p}\|^{2} c^{2}=E_{0}(v)^{2}
$$

Even if we make the additional and rather stringent assumption that rest masses are invariant, independent of absolute velocity through the aether, (and this is very definitely an extra assumption beyond reciprocity), one still picks up non-trivial 
physics via the $v$-dependent function $\zeta(v)$ :

$$
E=\frac{E_{0}}{\sqrt{1-\zeta(v) v^{2} / c^{2}}} ; \quad \boldsymbol{p}=\frac{\zeta(v) E_{0} \boldsymbol{v} / c^{2}}{\sqrt{1-\zeta(v) v^{2} / c^{2}}}=E \boldsymbol{v} / c^{2}
$$

with

$$
E^{2}-\zeta^{-1}(v)\|\boldsymbol{p}\|^{2} c^{2}=E_{0}^{2} .
$$

This model is the second of the "minimalist" models of Lorentz violation we refer to in the introduction. It is particularly useful in that it gives one a very clean specific "target" to take aim at.

\subsubsection{Linearity plus isotropy plus reciprocity plus relativity}

If we now (finally) adopt the relativity principle, then for arbitrary $\boldsymbol{v}_{1}$ and $\boldsymbol{v}_{2}$ the object $M\left(\boldsymbol{v}_{2}, \boldsymbol{v}_{1}\right)$ must equal $M(\boldsymbol{w})$ for some $\boldsymbol{w}\left(\boldsymbol{v}_{1}, \boldsymbol{v}_{2}\right)$ (with $\boldsymbol{w}$ being interpreted as the relative velocity of the two inertial frames). But this then implies that the set $\{M(\boldsymbol{v})\}$ forms a group, not merely a groupoid/pseudogroup. We shall see that this group condition implies $\zeta=1$, whence finally $\gamma=1 / \sqrt{1-v^{2} / c^{2}}$. But $c$ was some arbitrary quantity with the dimensions of velocity, it was not pre-judged to be the physical speed of light. Finite $c$ gives you the Lorentz group, infinite $c$ gives the Galileo group. (And the exceptional case $c^{2}<0$ actually means one is in Euclidean signature, and one obtains the $S O(4)$ rotation group. This exceptional case is normally excluded by appeal to a "pre-causality" principle [199].)

As an explicit check, assuming linearity+isotropy+reciprocity we have for two transformations $M_{1}$ and $M_{2}$

$$
\begin{aligned}
& M_{1}=\left[\begin{array}{c|c|c}
\gamma_{1} & -\gamma_{1} \zeta_{1} v_{1} / c^{2} & \mathbf{0}^{T} \\
\hline-\gamma_{1} v_{1} & \gamma_{1} & \mathbf{0}^{T} \\
\hline \mathbf{0} & \mathbf{0} & I
\end{array}\right] ; \\
& M_{2}=\left[\begin{array}{c|c|c}
\gamma_{2} & -\gamma_{2} \zeta_{2} v_{2} / c^{2} & \mathbf{0}^{T} \\
\hline-\gamma_{2} v_{2} & \gamma_{2} & \mathbf{0}^{T} \\
\hline \mathbf{0} & \mathbf{0} & I
\end{array}\right] ;
\end{aligned}
$$

subject to the constraint

$$
\gamma_{i}=\frac{1}{\sqrt{1-\zeta_{i} v_{i}^{2} / c^{2}}} .
$$

Then the group property requires the existence of some $v_{12}$ such that

$$
M_{1} M_{2}=M_{12}
$$


Explicitly:

$$
\begin{aligned}
& {\left[\begin{array}{c|c|c}
\gamma_{1} \gamma_{2}\left(1+\zeta_{1} v_{1} v_{2} / c^{2}\right) & -\gamma_{1} \gamma_{2}\left(\zeta_{1} v_{1}+\zeta_{2} v_{2}\right) / c^{2} & \mathbf{0}^{T} \\
\hline-\gamma_{1} \gamma_{2}\left(v_{1}+v_{2}\right) & \gamma_{1} \gamma_{2}\left(1+\zeta_{2} v_{1} v_{2} / c^{2}\right) & \mathbf{0}^{T} \\
\hline \mathbf{0} & \mathbf{0} & I
\end{array}\right]} \\
& =\left[\begin{array}{c|c|c}
\gamma_{12} & -\gamma_{12} \zeta_{12} v_{12} / c^{2} & \mathbf{0}^{T} \\
\hline-\gamma_{12} v_{12} & \gamma_{12} & \mathbf{0}^{T} \\
\hline \mathbf{0} & \mathbf{0} & I
\end{array}\right] .
\end{aligned}
$$

But by comparing the diagonal elements this can be true only if $\zeta_{1}=\zeta_{2}$ for all values of $v_{1}$ and $v_{2}$. That is, $\zeta\left(v_{1}\right)=\zeta\left(v_{2}\right)$ for all values of $v_{1}$ and $v_{2}$, so that there exists some velocity independent constant $\zeta_{0}$ such that

$$
\zeta(v)=\zeta_{0} .
$$

This now implies

$$
\begin{aligned}
& M_{1}=\left[\begin{array}{c|c|c}
\gamma_{1} & -\gamma_{1} \zeta_{0} v_{1} / c^{2} & \mathbf{0}^{T} \\
\hline-\gamma_{1} v_{1} & \gamma_{1} & \mathbf{0}^{T} \\
\hline \mathbf{0} & \mathbf{0} & I
\end{array}\right] \\
& M_{2}=\left[\begin{array}{c|c|c}
\gamma_{2} & -\gamma_{2} \zeta_{0} v_{2} / c^{2} & \mathbf{0}^{T} \\
\hline-\gamma_{2} v_{2} & \gamma_{2} & \mathbf{0}^{T} \\
\hline \mathbf{0} & \mathbf{0} & I
\end{array}\right] .
\end{aligned}
$$

The statement $M_{1} M_{2}=M_{12}$ becomes

$$
\begin{gathered}
{\left[\begin{array}{c|c|c}
\gamma_{1} \gamma_{2}\left(1+\zeta_{0} v_{1} v_{2} / c^{2}\right) & -\gamma_{1} \gamma_{2} \zeta_{0}\left(v_{1}+v_{2}\right) / c^{2} & \mathbf{0}^{T} \\
\hline-\gamma_{1} \gamma_{2}\left(v_{1}+v_{2}\right) & \gamma_{1} \gamma_{2}\left(1+\zeta_{0} v_{1} v_{2} / c^{2}\right) & \mathbf{0}^{T} \\
\hline \mathbf{0} & \mathbf{0} & I
\end{array}\right]} \\
=\left[\begin{array}{c|c|c}
\gamma_{12} & -\gamma_{12} \zeta_{0} v_{12} / c^{2} & \mathbf{0}^{T} \\
\hline-\gamma_{12} v_{12} & \gamma_{12} & \mathbf{0}^{T} \\
\hline \mathbf{0} & \mathbf{0} & I
\end{array}\right] .
\end{gathered}
$$

But now we can simply absorb $\zeta_{0}$ into a redefinition of $c$. After all, $c$ is at this stage just an arbitrary but fixed constant with the dimensions of velocity. Taking $c^{2} \rightarrow c^{2} / \zeta_{0}$ we have

$$
M_{1}=\left[\begin{array}{c|c|c}
\gamma_{1} & -\gamma_{1} v_{1} / c^{2} & \mathbf{0}^{T} \\
\hline-\gamma_{1} v_{1} & \gamma_{1} & \mathbf{0}^{T} \\
\hline \mathbf{0} & \mathbf{0} & I
\end{array}\right]
$$




$$
M_{2}=\left[\begin{array}{c|c|c}
\gamma_{2} & -\gamma_{2} v_{2} / c^{2} & \mathbf{0}^{T} \\
\hline-\gamma_{2} v_{2} & \gamma_{2} & \mathbf{0}^{T} \\
\hline \mathbf{0} & \mathbf{0} & I
\end{array}\right]
$$

Therefore

$$
\begin{array}{r}
M_{1} M_{2}=\left[\begin{array}{c|c|c}
\gamma_{1} \gamma_{2}\left(1+v_{1} v_{2} / c^{2}\right) & -\gamma_{1} \gamma_{2}\left(v_{1}+v_{2}\right) / c^{2} & \mathbf{0}^{T} \\
\hline-\gamma_{1} \gamma_{2}\left(v_{1}+v_{2}\right) & \gamma_{1} \gamma_{2}\left(1+v_{1} v_{2} / c^{2}\right) & \mathbf{0}^{T} \\
\hline \mathbf{0} & \mathbf{0} & I
\end{array}\right] \\
=\left[\begin{array}{c|c|c}
\gamma_{12} & -\gamma_{12} v_{12} / c^{2} & \mathbf{0}^{T} \\
\hline-\gamma_{12} v_{12} & \gamma_{12} & \mathbf{0}^{T} \\
\hline \mathbf{0} & \mathbf{0} & I
\end{array}\right] .
\end{array}
$$

If $c^{2}$ is finite and positive, we have the Lorentz transformations. If $c^{2}$ is infinite we have Galileo's transformations. This is (essentially) von Ignatowsky's result. (Note that $c^{2}=0$ is hopelessly diseased ${ }^{2}$ while $c^{2}<0$ actually corresponds to Euclidean signature space-time, with the set $\{M\}$ being the group $S O(4)$ of Euclidean rotations.)

\subsection{Conclusions regarding the relativity principle}

We have seen that once one for any reason moves away from Lorentz invariance, and specifically once one discards the relativity principle, then many of the intuitions one has been trained to develop in a special relativistic setting need to be significantly and carefully revised. In the next chapter we shall consider threshold phenomena, also appearing in [46], which can be studied by picking and working in a particular arbitrary but fixed inertial frame. In this chapter we have carefully analysed what happens to the transformation properties between inertial frames once the relativity principle is abandoned. A key message to take from the above is that the situation is not hopeless - even in the absence of a relativity principle quite a lot can still be said regarding the transformation properties between inertial frames, the combination of 3 -velocities, the transformation of 4-momenta, and the interplay between the energymomentum relations for on-shell particles and the transformation properties between inertial frames.

\footnotetext{
${ }^{2}$ It is at this stage, setting $c^{2} \rightarrow 0$, that one could if desired obtain Carroll kinematics 95, 103, 134, 155, 195. by enforcing the particular limit $c^{2} \rightarrow 0$, while $v \rightarrow 0$, but holding the slowness $u=v / c^{2}$ fixed. The relevance to "real world" physics seems somewhat tenuous.
} 
Key features of the analysis are the groupoid/pseudogroup structure of the set of transformations, the fact that 4-momentum transforms affinely as a dual vector, the fact that there are a number of distinct stages by which Lorentz invariance can be recovered - by successively imposing linearity, then isotropy, then reciprocity, and finally the relativity principle. The net result is a coherent framework within which Lorentz symmetry breaking can be explored in a controlled and internally consistent manner, while retaining usual notions of local physics. Overall the results of this chapter and of the following one, provide general techniques of interest when analysing the specific sector of physics that could present some Lorentz symmetry breaking (e.g. the neutrinos). These results provide general techniques of interest for handling large classes of Lorentz-violating but local physical models. 


\section{Chapter 5}

\section{Lorentz violating kinematics: Threshold theorems}

\subsection{Introduction}

An important technical issue that considerably complicates detailed calculations in a Lorentz symmetry breaking scenario, is that once this invariance is violated the analysis of thresholds in both scattering and decay processes becomes extremely subtle, with many new and naively unexpected effects. In the current chapter we develop several extremely general threshold theorems that depend only on the existence of some energy momentum relation $E(\boldsymbol{p})$, eschewing even assumptions of isotropy or monotonicity. We shall argue that there are physically interesting situations where such a level of generality is called for, and that existing (partial) results in the literature make unnecessary technical assumptions. Even in this most general of settings, we show that at threshold all final state particles move with the same 3 -velocity, while initial state particles must have 3 -velocities parallel/anti-parallel to the final state particles. In contrast the various 3 -momenta can behave in a complicated and quite counter-intuitive manner.

Regarding this matter, the reader would certainly remember the results presented by the OPERA collaboration concerning some indications of "faster than light" neutrinos [5], (see also earlier more tentative results from the MINOS collaboration [6]). Even though proved to be caused by a flaw in the experimental apparatus, nevertheless this result reinvigorated the field and prompted the production of a few hundred theoretical papers. Notable contributions include [9, 10, 25, 69, 76, 79, 87, 92, 93, 102, 131, 136, 173, 175, 176, 205, 249, 293, 303. In addition to these very recent efforts, it is important to recognize that there is an older and extensive literature 
placing significant experimental and observational bounds on any possible violation of Lorentz invariance. See for instance work by Coleman and Glashow [88, 89], Jacobson and collaborators [158 165, 198, 211], and especially the Living Review by Mattingly [210]. Other theoretical frameworks for characterizing possible violations of Lorentz invariance include those of Nielsen and collaborators [80, 214 217], Kostelecky and collaborators [59, 191, 180 186], and the flat-space non-gravity framework developed by Anselmi [28 40]. Additionally, the Hořava gravity framework [156] naturally includes Lorentz violation [261, 262, 279 281,297 .

One of the key results in the literature devoted to possible violations of Lorentz invariance is that the normal intuition one develops regarding threshold phenomena requires significant modification. See for instance the articles by Coleman and Glashow [88, 89, and Jacobson, Liberati, and Mattingly [161, 211] — and the more recent follow-ups by Cohen and Glashow [87], and Liberati, Mattingly, and Maccione [205], focussing specifically on the OPERA results.

\section{Some assumptions}

In this chapter we shall generalise the analysis of threshold phenomena presented in the above mentioned works. We shall consider both single-particle decay processes, and two-particle scattering processes (possibly inelastic), taking care to make an absolute minimum of technical assumptions - thus greatly generalising previous analyses. Specifically:

- We will explicitly assume a normal space-time manifold based on $\mathbb{R}^{4}$. (This excludes, for instance, both non-commutative space-times and certain versions of DSR [172, 200].)

- We shall explicitly assume conservation of both energy and momentum.

- Furthermore we shall explicitly assume a Hamiltonian/Lagrangian framework, so that in view of Noether's theorem (combined with energy-momentum conservation) we are working in a homogeneous space-time. Specifically, we assume the free-particle energy to be some function of the 3-momentum, $E(\boldsymbol{p})$, and that this can be related to a 3 -velocity via Hamilton's equations

$$
\dot{\mathrm{x}}=\frac{\partial E}{\partial \boldsymbol{p}} ; \quad \dot{\boldsymbol{p}}=0 .
$$

(This is again a purely pragmatic decision based on the fact that we want to have a sufficiently well-defined framework in which to be able to say something reasonably concrete.) 
- We shall eschew any particular functional form for $E(\boldsymbol{p})$, though we will impose smoothness and differentiability constraints as needed.

- We shall explicitly not assume isotropy.

- Even if physics happens to be isotropic in the preferred ("aether") frame implicit in many specific Lorentz violating theories, there is no particular reason to assume isotropy of the energy-momentum relation in generic inertial frames. And since we do not necessarily know what the observer's 3 -velocity is with respect to the preferred frame, it is more useful to develop threshold analysis for generic observers in an explicitly observerdependent manner.

- Even as early as the 1980's attempts were made to take lattice physics seriously as a physical cutoff — with proton decay taking place with outgoing decay products preferentially aligned along the principal axes of the universe. In such a situation one would not have isotropy even in the preferred frame.

- Many of the "analogue space-time" models permit energy-momentum relations that have odd and possibly anisotropic behaviors at ultra-high energies [56, 276].

- Consider a generic quasiparticle propagating in a generic atomic lattice. (For instance, a conduction-band dressed electron.) The band structure will typically not be isotropic, even in the rest frame of the lattice. So if you are a condensed matter physicist, you will have no choice, you will simply have to acknowledge that non-isotropy of the energy-momentum relation is quite common, which will unavoidably influence your ability to analyze reaction thresholds.

- We shall also be extremely cautious concerning "monotonicity" assumptions - carefully formulating an appropriate concept of monotonicity, and carefully analyzing what can and cannot be extracted from such an assumption.

Because of the generality of these assumptions, our results will have considerably wider validity than the results currently extant in the literature. Even in this most general of frameworks, several rigorous theorems can be extracted. We shall show that at threshold all final state particles move with the same 3-velocity, while initial state particles must have 3 -velocities parallel/anti-parallel to the final state particles. In contrast the various 3-momenta can behave in quite complicated and counterintuitive fashion, and the 3-momenta need not even be collinear. 


\subsection{General background}

In this section we shall review some of the models already existing in the field, in order to comprehend which are their characteristics and limitations. In the ColemanGlashow analysis [89], most of the discussion is explicitly limited to the rather special case of single-particle decay processes where the initial and final state particles $(i \in\{1,2, \ldots, n\})$ all have energies of the form

$$
E_{i}\left(\boldsymbol{p}_{i}\right)=\sqrt{E_{i, 0}^{2}+\left\|\boldsymbol{p}_{i}\right\|^{2} c_{i}^{2}} .
$$

Here the "speed of light" can be particle dependent. (See especially equation (2.19) in reference [89]. A similar assumption is implicitly made in [88].) But it is reasonably clear that much of the discussion of thresholds in [89] would work for any generic $E_{i}\left(\boldsymbol{p}_{i}\right)$. In contrast, in the OPERA-related analysis of [87, this specific choice of energy-momentum relation is implicit, not explicit, but is absolutely essential to that discussion - see [205] for a generalization.

The Mattingly, Jacobson, and Liberati threshold analysis [211] focusses on energymomentum relations that are (even in their most general setting) taken to be both isotropic and monotonic. Specific examples are taken to be of the form

$$
E_{i}\left(\boldsymbol{p}_{i}\right)=\sqrt{E_{i, 0}^{2}+\left\|\boldsymbol{p}_{i}\right\|^{2} c^{2}+\eta_{i}\left(\left\|\boldsymbol{p}_{i}\right\| c\right)^{n} E_{*}^{2-n}} .
$$

Here $\eta_{i}$ is a dimensionless parameter and $E_{*}$ is an energy scale characterizing the deviations from Lorentz invariance. The special case $n=2$ corresponds to the Coleman-Glashow energy-momentum relation. If $\eta_{i}<0$ and $n>2$ then these energy-momentum relations can in principle exhibit a maximum — the energy "saturates" - this is a specific example of a much more general phenomenon:

- In lattice QFT regularizations energy-momentum relations are typically of the form

$$
E(\boldsymbol{p})=\sqrt{E_{0}^{2}+\left(\frac{\hbar c}{a}\right)^{2}\left\{\sin ^{2}\left(\frac{\boldsymbol{p}_{x} a}{\hbar}\right)+\sin ^{2}\left(\frac{\boldsymbol{p}_{y} a}{\hbar}\right)+\sin ^{2}\left(\frac{\boldsymbol{p}_{z} a}{\hbar}\right)\right\}},
$$

or, (for "massless" particles),

$$
E(\boldsymbol{p})=\frac{\hbar c}{a} \sqrt{\sin ^{2}\left(\frac{\boldsymbol{p}_{x} a}{\hbar}\right)+\sin ^{2}\left(\frac{\boldsymbol{p}_{y} a}{\hbar}\right)+\sin ^{2}\left(\frac{\boldsymbol{p}_{z} a}{\hbar}\right)},
$$

and typically exhibit a maximum energy $E_{\max }=\sqrt{E_{0}^{2}+3 \hbar^{2} c^{2} / a^{2}}$ in terms of the lattice spacing $a$. (One usually considers "small" momenta, $\|\boldsymbol{p}\| a / \hbar \ll 1$, where Lorentz invariance is approximately recovered. Herein the focus will be on deviations from Lorentz invariance.) 
- Qualitatively similar effects occur for quasiparticles propagating through atomic lattices - momentum space [the first Brillouin zone] is now compact, and so (assuming continuity) the energy will be bounded by some maximum.

- In DSR-inspired models, insofar as they can be incorporated into the current framework, one often has individual particle energies saturating at or around the Planck energy [172, 200].

- For a specific example (physically unmotivated but mathematically tractable) of saturation behaviour one might take

$$
E(\boldsymbol{p})=\sqrt{E_{0}^{2}+E_{*}^{2} \tanh \left(\frac{\|\boldsymbol{p}\|^{2} c^{2}}{E_{*}^{2}}\right)} .
$$

At low momentum this is approximately Lorentz invariant

$$
E(\boldsymbol{p}) \approx \sqrt{E_{0}^{2}+\|\boldsymbol{p}\|^{2} c^{2}+\mathcal{O}\left(\|\boldsymbol{p}\|^{4}\right)},
$$

but at high momentum it exponentially saturates

$$
E(\boldsymbol{p}) \approx \sqrt{E_{0}^{2}+E_{*}^{2}+\mathcal{O}\left(\exp \left(-2\|\boldsymbol{p}\|^{2} c^{2} / E_{*}^{2}\right)\right)} .
$$

- Neither the usual Lorentz invariant energy-momentum relation, nor even the Galilean invariant energy-momentum relation, saturate with a maximum energy. For that matter, neither does the Coleman-Glashow energy-momentum relation (5.2) saturate.

Since our purpose is to generalise threshold analysis as far as possible to situations where Lorentz invariance is broken, we will for generality entertain the possibility of energy-momentum relations that saturate to some maximum energy — with the understanding that this maximum energy might, in specific situations, be infinite. With this general framework in place we are now ready to begin detailed analysis.

\subsection{Cautionary comments}

Some cautionary comments are in order:

- For the lattice-like energy-momentum relation of equation (5.4) we have

$$
\boldsymbol{v}=\frac{\partial E}{\partial \boldsymbol{p}}=\frac{\hbar c}{2 E a}\left(\sin \left(\frac{2 \boldsymbol{p}_{x} a}{\hbar}\right), \sin \left(\frac{2 \boldsymbol{p}_{y} a}{\hbar}\right), \sin \left(\frac{2 \boldsymbol{p}_{z} a}{\hbar}\right)\right) .
$$

The key point is that 3-velocity $\boldsymbol{v}$ and 3-momentum $\boldsymbol{p}$ need not be parallel. Additionally $\boldsymbol{v}$ can exhibit non-trivial zeros for non-zero momentum $\boldsymbol{p}$, and 
even once one specifies a particular particle the inverse function $\boldsymbol{p}(\boldsymbol{v})$ can easily be (and typically is) multivalued. Such phenomena are not limited to the specific energy-momentum relation of equation (5.4), but rather are generic to any quasiparticle propagating through a regular lattice (for example, a conduction-band dressed electron).

- Non uniqueness of the inverse function $\boldsymbol{p}(\boldsymbol{v})$ is also generic for (higher than quadratic) polynomial or rational polynomial energy-momentum relations - it is the unique invertability of the Lorentz invariant energy-momentum relation $E=\sqrt{E_{*}^{2}+\|\boldsymbol{p}\|^{2} c^{2}}$ that is non generic in this regard. (Details depend on the precise values of the coefficients as the possibility of multi-valued behaviour depends on the root structure.)

- For the tanh-like energy-momentum relation of equation (5.6) we have

$$
\boldsymbol{v}=\frac{\partial E}{\partial \boldsymbol{p}}=c \operatorname{sech}^{2}\left(\frac{\|\boldsymbol{p}\|^{2} c^{2}}{E_{*}^{2}}\right) \frac{\boldsymbol{p} c}{E} .
$$

While 3-velocity $\boldsymbol{v}$ and 3-momentum $\boldsymbol{p}$ are now parallel, zero 3-velocity can correspond either to zero 3-momentum or to infinite 3-momentum (with finite energy $\left.\sqrt{E_{0}^{2}+E_{*}^{2}}\right)$. Low-velocity physics can thus be grossly misleading two particles with the same 3-velocity may have wildly differing 3-momenta. Such phenomena are not limited to the specific energy-momentum relation of equation (5.6), but rather are generic to any situation where the energy saturates as a function of 3-momentum. Note in particular that the energymomentum relation of equation (5.6) is monotonic - monotonicity is not enough to prevent this sort of behaviour. Similar behaviour also occurs whenever $\lim _{\|\boldsymbol{p}\| \rightarrow \infty} \partial E / \partial \boldsymbol{p} \rightarrow \mathbf{0}$, corresponding to a sub-linear asymptotic growth in the energy-momentum relation.

- Other unusual possibilities include energy minima occurring at non-zero 3momentum, (by definition an energy minimum must always occur at zero 3-velocity). Let $\mathbf{n}$ be an arbitrary unit vector and consider for instance

$$
E=\sqrt{E_{0}^{2}+\|\boldsymbol{p}\|^{2} c^{2}+k_{4}(\boldsymbol{p} \cdot \mathbf{n})^{4}+k_{6}(\boldsymbol{p} \cdot \mathbf{n})^{6}} .
$$

This energy-momentum relation is not only anisotropic, but by taking $k_{4}<0$ and $k_{6}>0$ one can arrange for a global minimum energy at some $\boldsymbol{p}_{\min }=$ $p_{\min } \mathbf{n} \neq \mathbf{0}$. The 3 -velocity is

$$
\boldsymbol{v}=\frac{\boldsymbol{p} c^{2}+2 k_{4}(\boldsymbol{p} \cdot \mathbf{n})^{3} \mathbf{n}+3 k_{6}(\boldsymbol{p} \cdot \mathbf{n})^{5} \mathbf{n}}{E}
$$


and $\boldsymbol{p}$ and $\boldsymbol{v}$ are generally not collinear (unless one happens to be considering motion parallel or perpendicular to the preferred axis $\mathbf{n}$.)

- As a final pedagogical example consider the isotropic energy-momentum relation

$$
E=\sqrt{E_{0}^{2}+\|\boldsymbol{p}\|\left\|^{2} c^{2}+k_{4}\right\| \boldsymbol{p}\left\|^{4}+k_{6}\right\| \boldsymbol{p} \|^{6}}
$$

By taking $k_{4}<0$ and $k_{6}>0$ one can arrange for a global minimum energy at some finite $\left\|\boldsymbol{p}_{\text {min }}\right\|=p_{\min } \neq 0$. The 3 -velocity is

$$
\boldsymbol{v}=\frac{\|\boldsymbol{p}\| c^{2}+2 k_{4}\|\boldsymbol{p}\|^{3}+3 k_{6}\|\boldsymbol{p}\|^{5}}{E} \hat{\boldsymbol{p}} .
$$

In this situation $\boldsymbol{p}$ and $\boldsymbol{v}$ are generally collinear, but whenever there is a global energy minimum at some finite $\left\|\boldsymbol{p}_{\min }\right\|=p_{\min } \neq 0$ there will also be a nonempty range of momenta for which $\boldsymbol{p}$ and $\boldsymbol{v}$ are anti-parallel.

These are merely five specific examples of the unusual behaviour one might potentially encounter, and the types of issues we shall potentially need to consider in our analysis.

\subsection{Decay thresholds}

Consider the decay process

$$
X_{0} \rightarrow X_{1}+X_{2}+\ldots X_{n}
$$

where for each individual particle we have the 4-momenta

$$
P_{i}=\left(E_{i}(\boldsymbol{p}), \boldsymbol{p}_{i}\right) .
$$

$\boldsymbol{p}_{i}$ and $E_{i}$ are respectively the out-going three-momentum and energy for each particle. We shall now study the kinematics of this decay process.

\subsubsection{Kinematically allowed region}

Let us define

$$
E_{\text {out }}\left(\boldsymbol{p}_{1}, \boldsymbol{p}_{2}, \ldots, \boldsymbol{p}_{n}\right)=\sum_{i=1}^{n} E_{i}\left(\boldsymbol{p}_{i}\right)
$$

and

$$
\mathcal{P}_{\text {out }}\left(\boldsymbol{p}_{0}\right)=\left\{\left(\boldsymbol{p}_{1}, \boldsymbol{p}_{2}, \ldots, \boldsymbol{p}_{n}\right): \sum_{i=1}^{n} \boldsymbol{p}_{i}=\boldsymbol{p}_{0}\right\} .
$$


Then $\mathcal{P}_{\text {out }}\left(\boldsymbol{p}_{0}\right)$ is a collection of three individually connected hyperplanes in $\mathbb{R}^{n}$, one hyperplane for each Cartesian component of $\boldsymbol{p}_{0}$, corresponding to the set of all possible outgoing 3 -momenta for fixed total 3 -momentum $\boldsymbol{p}_{0}$. Thus $\mathcal{P}_{\text {out }}\left(\boldsymbol{p}_{0}\right)$ is a $3 n-3$ dimensional plane (affine subspace) of co-dimension 3 in $\mathbb{R}^{3 n}$, and is both convex and connected as a subset of $\mathbb{R}^{3 n}$. But the individual $E_{i}\left(\boldsymbol{p}_{i}\right)$ are by assumption differentiable and continuous, so $E_{\text {out }}\left(\boldsymbol{p}_{1}, \boldsymbol{p}_{2}, \ldots, \boldsymbol{p}_{n}\right)$ is also differentiable and continuous. In particular, since $\mathcal{P}_{\text {out }}\left(\boldsymbol{p}_{0}\right)$ is connected, this implies the image $E_{\text {out }}\left(\mathcal{P}_{\text {out }}\left(\boldsymbol{p}_{0}\right)\right)$ is a connected interval in $\mathbb{R}$.

For specified initial 3 -momentum $\boldsymbol{p}_{0}$, the decay process 5.15 is kinematically allowed if and only if

$$
E_{0}\left(\boldsymbol{p}_{0}\right) \in E_{\text {out }}\left(\mathcal{P}_{\text {out }}\left(\boldsymbol{p}_{0}\right)\right) .
$$

That is, the decay is allowed if and only if among the set of all possible output 3-momenta $\left\{\boldsymbol{p}_{1}, \boldsymbol{p}_{2}, \ldots, \boldsymbol{p}_{n}\right\}$ that conserve total 3-momentum, there is at least one configuration that also conserves total energy. We could also phrase the kinematically allowed region in terms of an allowable set of output momenta by considering the inverse image

$$
E_{\text {out }}^{-1}\left(E_{0}\left(\boldsymbol{p}_{0}\right)\right) \cap \mathcal{P}_{\text {out }}\left(\boldsymbol{p}_{0}\right),
$$

a set which, for given $\boldsymbol{p}_{0}$, may or may not be empty. Alternatively one can ask the question

$$
\boldsymbol{p}_{0} \in E_{0}^{-1}\left(E_{\text {out }}\left(\mathcal{P}_{\text {out }}\left(\boldsymbol{p}_{0}\right)\right)\right) ?
$$

But these approaches in terms of inverse images can be somewhat clumsy.

As a more practical way to better characterize the kinematically allowed region, it is useful to introduce the two quantities

$$
E_{\min }\left(\boldsymbol{p}_{0}\right)=\min E_{\text {out }}\left(\mathcal{P}_{\text {out }}\left(\boldsymbol{p}_{0}\right)\right)=\min \left\{\sum_{i=1}^{n} E_{i}\left(\boldsymbol{p}_{i}\right): \sum_{i=1}^{n} \boldsymbol{p}_{i}=\boldsymbol{p}_{0}\right\},
$$

and

$$
E_{\max }\left(\boldsymbol{p}_{0}\right)=\max E_{\text {out }}\left(\mathcal{P}_{\text {out }}\left(\boldsymbol{p}_{0}\right)\right)=\max \left\{\sum_{i=1}^{n} E_{i}\left(\boldsymbol{p}_{i}\right): \sum_{i=1}^{n} \boldsymbol{p}_{i}=\boldsymbol{p}_{0}\right\} .
$$

A more technically precise statement would use the concepts of supremum and infimum, but as long as we understand that statements made below might sometimes have to be interpreted in terms of suitable limits, such a level of precision is, for our purposes, unnecessary.

Then the decay process 5.15 is kinematically allowed if and only if

$$
E_{\min }\left(\boldsymbol{p}_{0}\right) \leq E_{0}\left(\boldsymbol{p}_{0}\right) \leq E_{\max }\left(\boldsymbol{p}_{0}\right) .
$$


A decay threshold is now defined to be the edge of the kinematically allowed region. Specifically, an enabling threshold is defined by the condition

$$
E_{\min }\left(\boldsymbol{p}_{0}\right)=E_{0}\left(\boldsymbol{p}_{0}\right)
$$

and a saturation threshold is defined by the condition

$$
E_{0}\left(\boldsymbol{p}_{0}\right)=E_{\max }\left(\boldsymbol{p}_{0}\right)
$$

These thresholds are typically 2 -surfaces in 3 -momentum space.

- Note that $E_{\max }\left(\boldsymbol{p}_{0}\right)$ might trivially be infinite if any one of the energy-momentum relations does not saturate at large 3-momentum, in which case no useful upper bound, and hence no saturation threshold, would be obtained. This is the case for instance in standard special relativity, in standard Galilean kinematics, in the Coleman-Glashow energy-momentum relation (5.2), and for $\eta>0$ in the Mattingly-Jacobson-Liberati energy-momentum relation (5.3.)

- Note that in standard special relativity the enabling threshold is also trivial one need merely go into the centre-of-momentum frame to see that the decay is kinematically allowed if and only if

$$
m_{0} \geq \sum_{n=1}^{n} m_{i}
$$

That is, decay thresholds are trivial in the case of exact Lorentz invariance, (see figure 5.1) and only become interesting if there are deviations from Lorentz invariance.

\subsubsection{Thresholds in momentum space}

For a graphical understanding of the situation it is useful to pick some (arbitrary but fixed) direction $\hat{\boldsymbol{p}}$ in momentum space, write $\boldsymbol{p}_{0}=p_{0} \hat{\boldsymbol{p}}$, and for each direction $\hat{\boldsymbol{p}}$ consider the three curves:

$$
\begin{aligned}
\mathcal{C}_{+}(\hat{\boldsymbol{p}}) & =\left\{E_{\max }\left(p_{0} \hat{\boldsymbol{p}}\right), p_{0}\right\} \\
\mathcal{C}_{0}(\hat{\boldsymbol{p}}) & =\left\{E_{0}\left(p_{0} \hat{\boldsymbol{p}}\right), p_{0}\right\} ; \\
\mathcal{C}_{-}(\hat{\boldsymbol{p}}) & =\left\{E_{\min }\left(p_{0} \hat{\boldsymbol{p}}\right), p_{0}\right\} .
\end{aligned}
$$

Note that if the individual energy-momentum relations are isotropic, (rotationally invariant, spherically symmetric), then these curves $\mathcal{C}_{+/ 0 /-}$ will be independent of the direction $\hat{\boldsymbol{p}}$. If Lorentz invariance is violated, isotropy would at best occur 


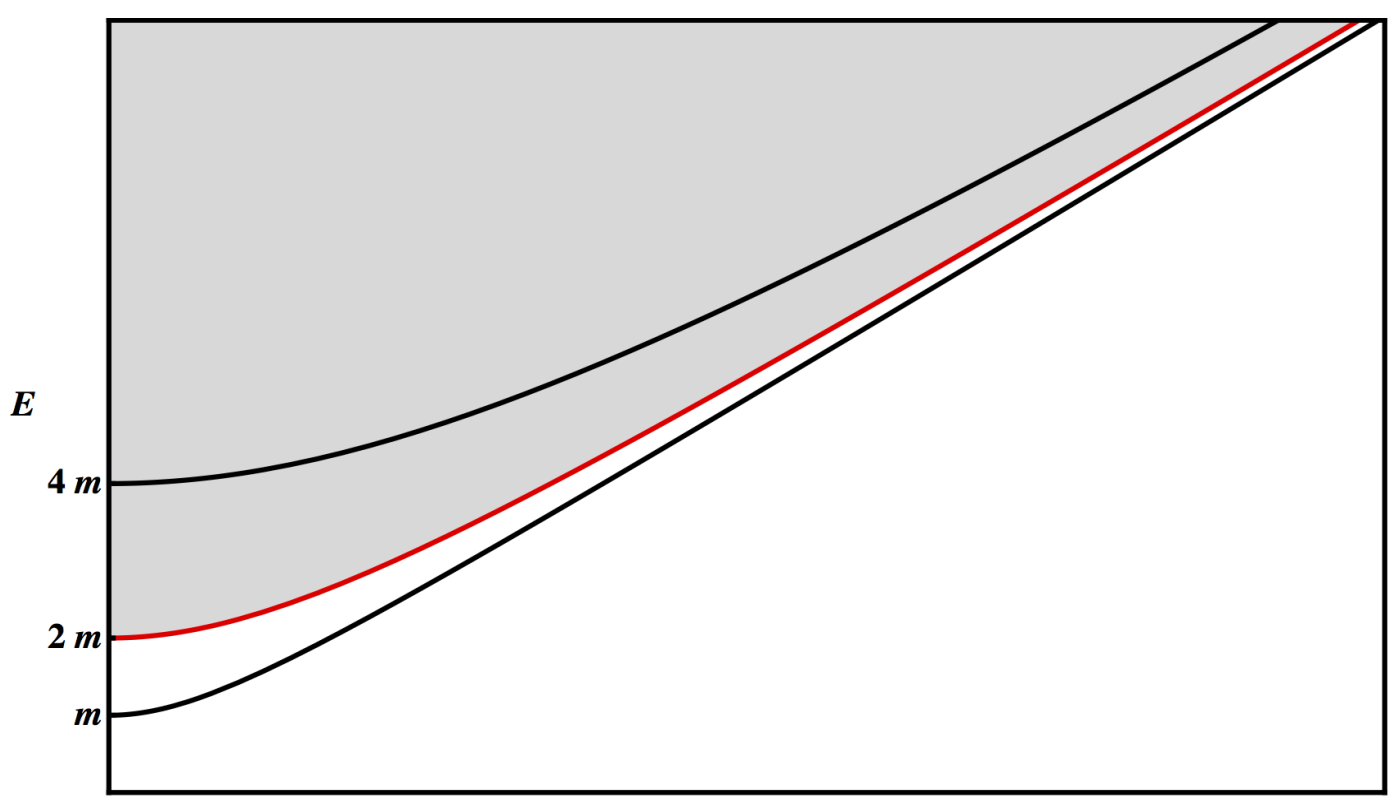

$p_{0}$

Figure 5.1: The shaded area shows the kinematically accessible region for a Lorentz invariant particle of mass $m$ (lower black line), and a Lorentz invariant particle of mass $4 m$ (upper black line), decaying to two identical particles of mass $m$ (red line). Note absence of decay thresholds: The process is either allowed or forbidden in a momentum-independent manner. 
only in the preferred (aether) frame, so in general it is safer to not make any such assumption.

The kinematically accessible region for the decay products (assuming only conservation of 3-momentum) is the region between the curves $\mathcal{C}_{-}$and $\mathcal{C}_{+}$. Kinematically allowed decays correspond to that portion of $\mathcal{C}_{0}$ that lies in the region between the curves $\mathcal{C}_{-}$and $\mathcal{C}_{+}$. Enabling thresholds occur whenever the curve $\mathcal{C}_{0}$ intersects the curve $\mathcal{C}_{-}$, saturation thresholds occur whenever the curve $\mathcal{C}_{0}$ intersects the curve $\mathcal{C}_{+}$, see figure 5.2

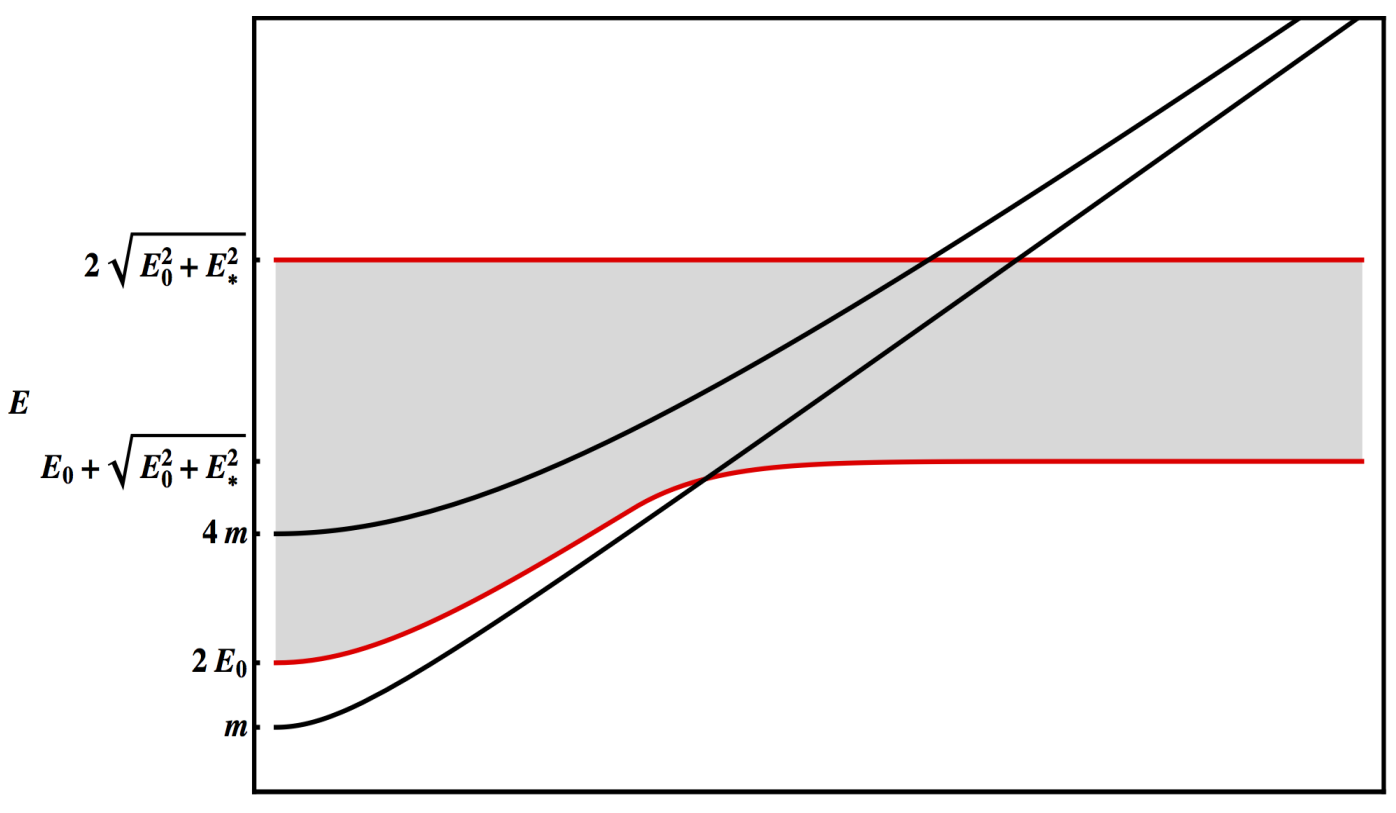

$p_{0}$

Figure 5.2: The kinematically accessible region for a Lorentz invariant particle of mass $m$ (lower black line), and a Lorentz invariant particle of mass $4 m$ (upper black line), decaying to two identical particles with "tanh-like" energy-momentum relation $E=\sqrt{E_{0}^{2}+E_{*}^{2} \tanh \left(p^{2} c^{2} / E_{*}^{2}\right)}$ (red lines). For the mass $m$ particle note the presence of both enabling and saturation thresholds (respectively the two points where the red lines and the lower black line cross). For the mass $4 m$ particle only the saturation threshold survives (where there upper black line and upper red line cross).

It is additionally useful to distinguish lower and upper thresholds. A lower threshold occurs when, as a function of increasing $p_{0}$, the curve $\mathcal{C}_{0}$ enters the kinematically accessible region, and an upper threshold occurs when, as a function of increasing $p_{0}$, the curve $\mathcal{C}_{0}$ leaves the kinematically accessible region.

There are some quite general results for the curves $\mathcal{C}_{ \pm}$that are not too difficult to establish. Consider for simplicity a 2-particle final state, or a 2-particle subsystem 


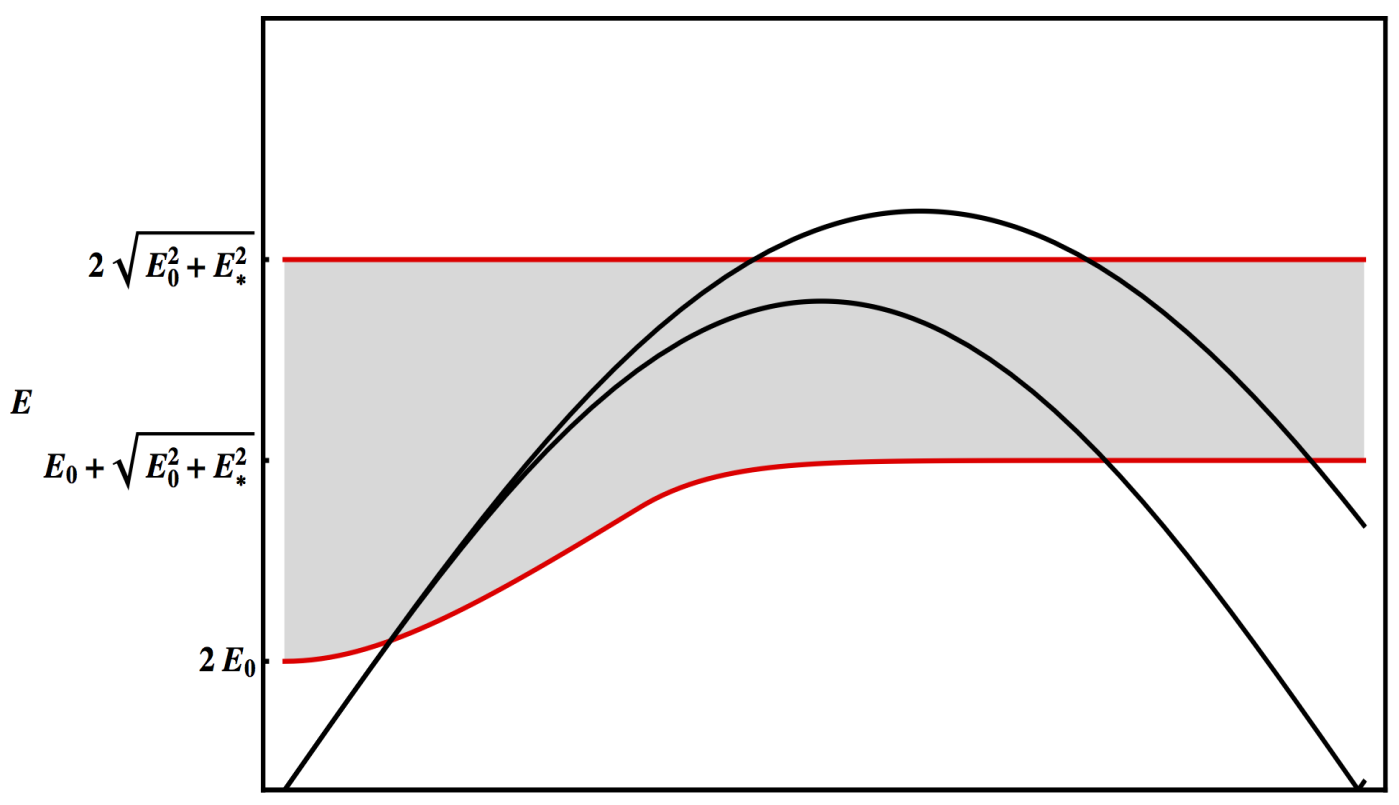

$p_{0}$

Figure 5.3: The kinematically accessible region for initial particles with a "lattice-like" energymomentum relation $E=(\hbar c / a)|\sin (p a / \hbar)|$, (with two distinct values of the "lattice spacing" $a$, black lines), decaying to two identical particles with "tanh-like" energy-momentum relation $E=$ $\sqrt{E_{0}^{2}+E_{*}^{2} \tanh \left(p^{2} c^{2} / E_{*}^{2}\right)}$ (two red lines). For small lattice spacing (the upper sine curve) note the presence of four thresholds: In order they are lower enabling, upper saturation, lower saturation, and upper enabling thresholds. For larger lattice spacing (the lower sine curve) only the lower enabling and upper enabling thresholds survive. 


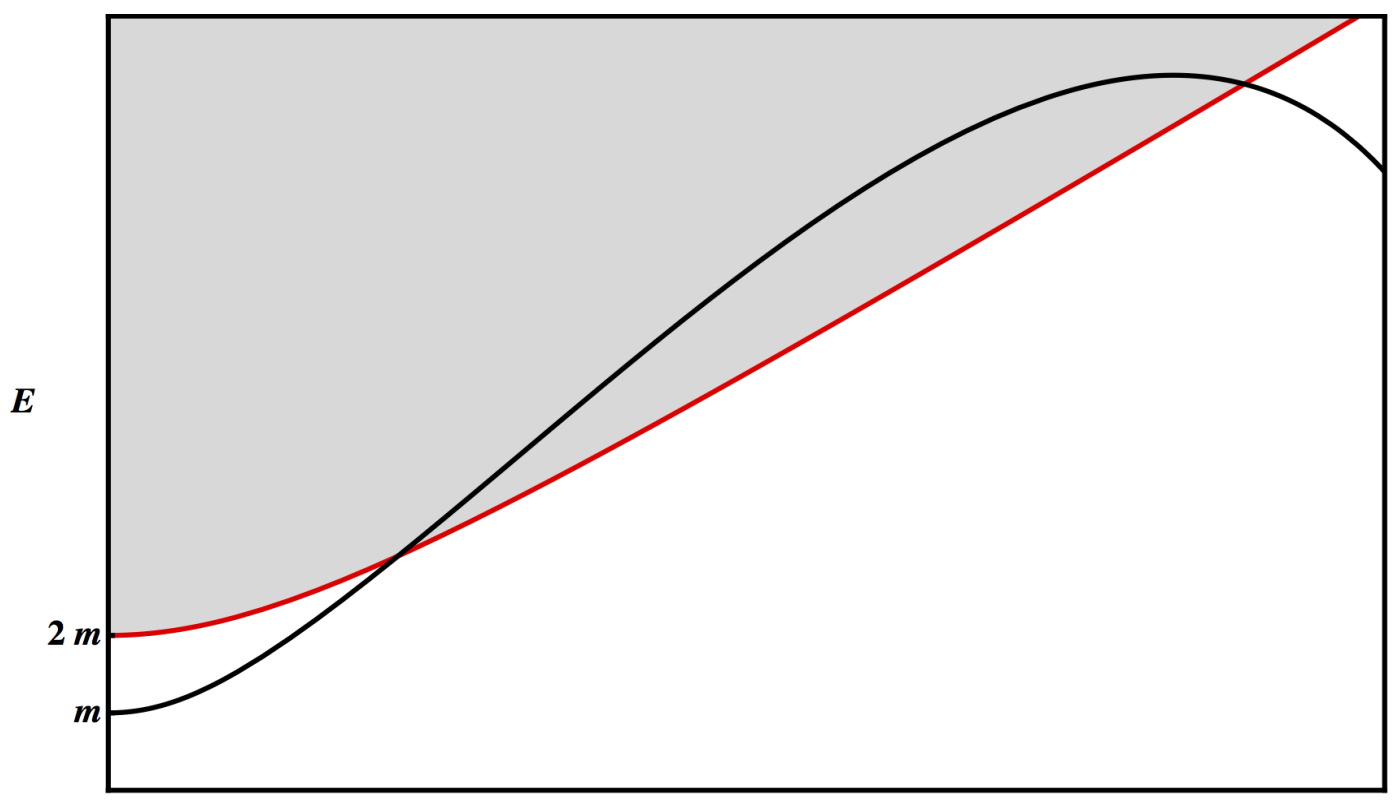

$p_{0}$

Figure 5.4: The kinematically accessible region for a particle with a mass $m$ and a polynomial energy-momentum relation (black line), decaying to two identical Lorentz invariant particles of mass $m$ (red line). Note presence of both lower and upper enabling thresholds, but no saturation thresholds. 


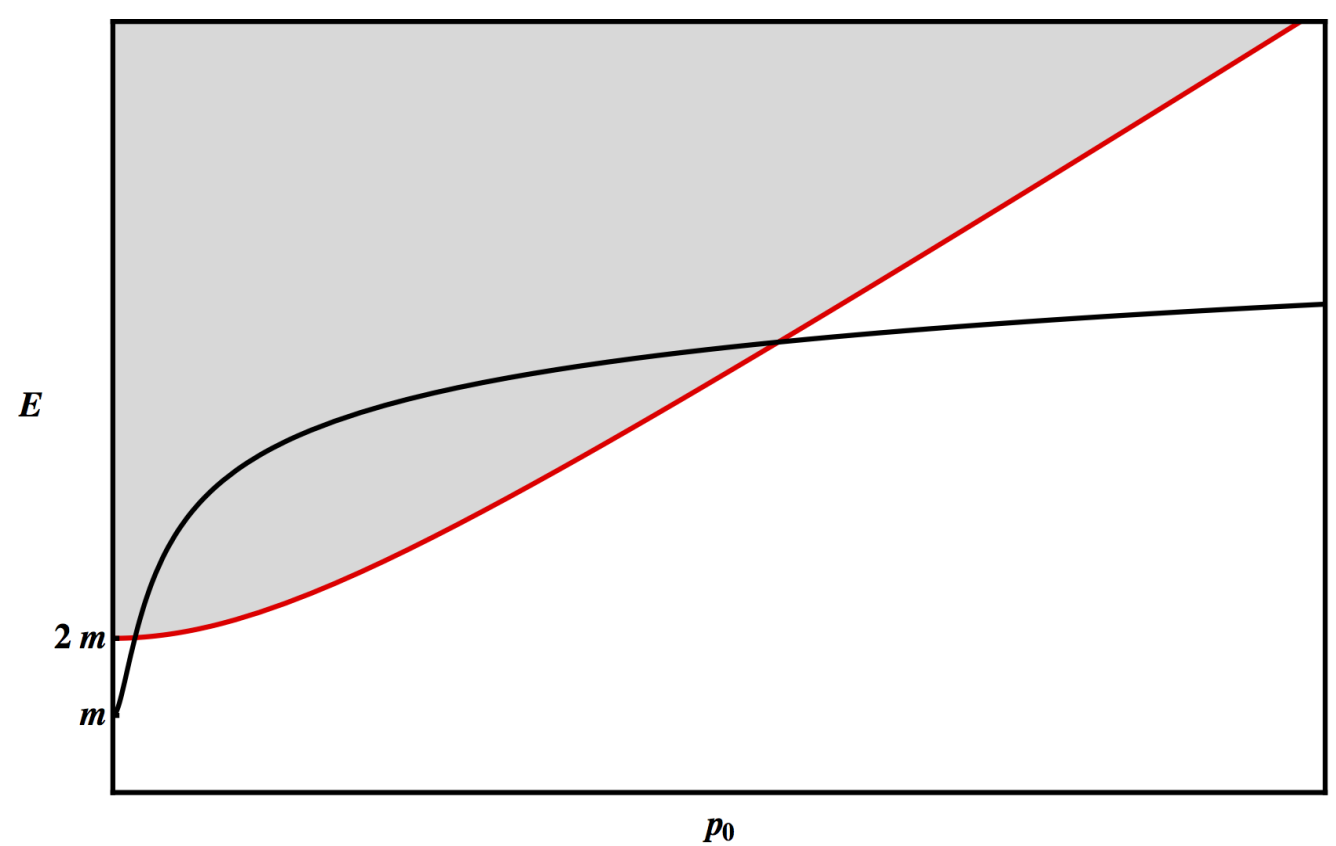

Figure 5.5: The kinematically accessible region for a particle of mass $m$ with a complicated but monotonic energy-momentum relation (black line), decaying to two identical Lorentz invariant particles of mass $m$ (red line). Note presence of both lower and upper enabling thresholds, but no saturation thresholds. 
of a $n$-body final state. Then consider the (restricted) set of curves

$$
\mathcal{C}_{\epsilon}(\hat{\boldsymbol{p}})=\left\{E_{1}\left(\epsilon p_{0} \hat{\boldsymbol{p}}\right)+E_{2}\left([1-\epsilon] p_{0} \hat{\boldsymbol{p}}\right), p_{0}\right\} \quad \forall \epsilon \in \mathbb{R},
$$

and (now with $\hat{\boldsymbol{p}} \cdot \hat{\boldsymbol{p}}_{\perp}=0$ ) the more general set of curves

$$
\mathcal{C}_{\epsilon, \delta}\left(\hat{\boldsymbol{p}}, \hat{\boldsymbol{p}}_{\perp}\right)=\left\{E_{1}\left(\epsilon p_{0} \hat{\boldsymbol{p}}+\delta \hat{\boldsymbol{p}}_{\perp}\right)+E_{2}\left([1-\epsilon] p_{0} \hat{\boldsymbol{p}}-\delta \hat{\boldsymbol{p}}_{\perp}\right), p_{0}\right\} \quad \forall \epsilon, \delta \in \mathbb{R} .
$$

All of these curves lie between $\mathcal{C}_{-}$and $\mathcal{C}_{+}$, and can be used to quickly sketch out the kinematically allowed region. In particular the curve

$$
\left\{E_{1}\left(p_{0} \hat{\boldsymbol{p}} / 2\right)+E_{2}\left(p_{0} \hat{\boldsymbol{p}} / 2\right), p_{0}\right\}
$$

corresponds to sharing momentum equally between the two particles, and so automatically lies between $\mathcal{C}_{-}$and $\mathcal{C}_{+}$. Perhaps less obviously the two curves

$$
\left\{E_{1}\left(p_{0} \hat{\boldsymbol{p}}\right)+E_{2}(\mathbf{0}), p_{0}\right\} \quad \text { and } \quad\left\{E_{1}(\mathbf{0})+E_{2}\left(p_{0} \hat{\boldsymbol{p}}\right), p_{0}\right\}
$$

correspond to putting all available 3-momentum into particle 1 or particle 2 respectively, and both these curves automatically lie between $\mathcal{C}_{-}$and $\mathcal{C}_{+}$. By considering the limits $\epsilon \rightarrow \pm \infty$ we also see that the two horizontal lines

$$
\left\{E_{1}( \pm \infty \hat{\boldsymbol{p}})+E_{2}(\mp \infty \hat{\boldsymbol{p}}), p_{0}\right\}
$$

lie in the kinematically allowed region. (These last two curves are most useful when the energy-momentum relations saturate at large 3-momentum.) Finally note that the vertical line

$$
\left\{E_{1}\left(p_{0} \hat{\boldsymbol{p}}\right)+E_{2}\left(-p_{0} \hat{\boldsymbol{p}}\right), 0\right\}
$$

also lies entirely within the kinematically allowed region. These observations allow one to quickly sketch key features of the kinematically allowed region. Some graphical experiments (see for instance figures 5.3, 5.4, and 5.5) will quickly convince one that in general the kinematically allowed region need not be convex, nor need the curves $\mathcal{C}_{ \pm}$necessarily be monotonic. If one is willing to make more specific assumptions concerning the energy-momentum relations, only then can much more be said about $\mathcal{C}_{ \pm}$and $E_{\max / \min }\left(\boldsymbol{p}_{0}\right)$. For instance:

- For the lattice energy-momentum relation of equation (5.4) we have $E_{i}\left(\boldsymbol{p}_{i}\right) \leq$ $\hbar c / a$, so provided all final state decay products see the same lattice, we have $E_{\max }\left(\boldsymbol{p}_{0}\right) \leq n \hbar c / a$, and so the curve $\mathcal{C}_{+}$will be nontrivial. 
- For the tanh-type energy-momentum relation of equation (5.6) it is easy to check that

$$
E_{\max }\left(\boldsymbol{p}_{0}\right)=\sum_{i=1}^{n} \sqrt{E_{0, i}^{2}+E_{*, i}^{2}}
$$

Then $\mathcal{C}_{+}$is a simple horizontal line. $\mathcal{C}_{-}$is however quite nontrivial, see figure 5.2 ,

Once one abandons isotropy - in particular azimuthal isotropy around the chosen direction $\hat{\boldsymbol{p}}$ in momentum space - then a fuller analysis using the curves $\mathcal{C}_{\epsilon, \delta}\left(\hat{\boldsymbol{p}}, \hat{\boldsymbol{p}}_{\perp}\right)$ will be necessary. Formally

$$
\mathcal{C}_{-}(\hat{\boldsymbol{p}})=\min _{\epsilon \in \mathbb{R}} \min _{\hat{\boldsymbol{p}}_{\perp}} \min _{\delta \in \mathbb{R}} \mathcal{C}_{\epsilon, \delta}\left(\hat{\boldsymbol{p}}, \hat{\boldsymbol{p}}_{\perp}\right),
$$

and

$$
\mathcal{C}_{+}(\hat{\boldsymbol{p}})=\max _{\epsilon \in \mathbb{R}} \max _{\hat{\boldsymbol{p}}_{\perp}} \max _{\delta \in \mathbb{R}} \mathcal{C}_{\epsilon, \delta}\left(\hat{\boldsymbol{p}}, \hat{\boldsymbol{p}}_{\perp}\right) .
$$

Typically the curves $\mathcal{C}_{ \pm}(\hat{\boldsymbol{p}})$ will piecewise consist of segments of some specific curves chosen from the $\mathcal{C}_{\epsilon, \delta}\left(\hat{\boldsymbol{p}}, \hat{\boldsymbol{p}}_{\perp}\right)$. The key message to extract from the discussion is this: Once exact Lorentz invariance is lost the kinematically allowed region can become extremely complicated.

\subsubsection{Lagrange multiplier techniques}

Further technical progress can best be made by introducing Lagrange multipliers and considering extrema (at fixed $\boldsymbol{p}_{0}$ ) of the function

$$
\mathcal{E}\left(\boldsymbol{p}_{0} ; \boldsymbol{p}_{i}, \boldsymbol{\lambda}\right)=\sum_{i=1}^{n} E_{i}\left(\boldsymbol{p}_{i}\right)-\boldsymbol{\lambda} \cdot\left(\sum_{i=1}^{n} \boldsymbol{p}_{i}-\boldsymbol{p}_{0}\right) .
$$

All minima used to determine $E_{\min }\left(\boldsymbol{p}_{0}\right)$, or maxima used to determine $E_{\max }\left(\boldsymbol{p}_{0}\right)$, will be extrema of the function $\mathcal{E}\left(\boldsymbol{p}_{0} ; \boldsymbol{p}_{i}, \boldsymbol{\lambda}\right)$ (though not necessarily vice versa). So extrema of $\mathcal{E}\left(\boldsymbol{p}_{0} ; \boldsymbol{p}_{i}, \boldsymbol{\lambda}\right)$ will provide information concerning thresholds.

But all extrema of $\mathcal{E}$ satisfy

$$
\frac{\partial E_{i}}{\partial \boldsymbol{p}_{i}}=\boldsymbol{\lambda}
$$

In view of the specific Hamilton equation $\boldsymbol{v}=\dot{\mathbf{x}}=\partial E / \partial \boldsymbol{p}$, this implies that at any extremum $\boldsymbol{v}_{i}=\boldsymbol{\lambda}=\boldsymbol{v}_{\text {out }}$ - all output velocities for the decay products are equal at any extremum, so in particular all output velocities are equal for the specific configuration of decay product 3 -momenta $\boldsymbol{p}_{i}$ that define $E_{\min }\left(\boldsymbol{p}_{0}\right)$ and $E_{\max }\left(\boldsymbol{p}_{0}\right)$. 
That is, we have the very general result that at threshold all final state particles move with the same 3-velocity. Furthermore for any extremum we also have

$$
\frac{\partial \mathcal{E}\left(\boldsymbol{p}_{0} ; \boldsymbol{p}_{i}, \boldsymbol{\lambda}\right)}{\partial \boldsymbol{p}_{0}}=\boldsymbol{\lambda}=\boldsymbol{v}_{\mathrm{out}},
$$

so in particular this will also be true for $E_{\min }\left(\boldsymbol{p}_{0}\right)$ and $E_{\max }\left(\boldsymbol{p}_{0}\right)$. That is: For the specific configuration of decay product 3-momenta $\boldsymbol{p}_{i}$ that define $E_{\min }\left(\boldsymbol{p}_{0}\right)$ and $E_{\max }\left(\boldsymbol{p}_{0}\right)$ we have

$$
\frac{\partial E_{\min }}{\partial \boldsymbol{p}_{0}}=\boldsymbol{v}_{\text {out }, \min }=\frac{\partial E_{i}}{\partial \boldsymbol{p}_{i}},
$$

and

$$
\frac{\partial E_{\max }}{\partial \boldsymbol{p}_{0}}=\boldsymbol{v}_{\text {out }, \max }=\frac{\partial E_{i}}{\partial \boldsymbol{p}_{i}},
$$

respectively. Physically this implies that at any threshold (regardless of whether it is an enabling threshold or a saturation threshold, or a lower or upper threshold) all decay products will be moving at the same physical 3 -velocity. This does not necessarily imply that the 3-momenta be related in any simple way, in general the 3 -momenta need not even be collinear. At threshold we can define the incoming 3 -velocity as

$$
\frac{\partial E_{0}}{\partial \boldsymbol{p}_{0}}=\boldsymbol{v}_{\text {in }},
$$

but with the techniques currently at hand there is in general no simple relation between $\boldsymbol{v}_{\text {in }}$ and $\boldsymbol{v}_{\text {out }}$. The best we can currently do is this: If we look along a particular direction $\hat{\boldsymbol{p}}$ in 3 -momentum space (with $\boldsymbol{p}_{0}=p_{0} \hat{\boldsymbol{p}}$ ) then:

- At a lower enabling threshold $\hat{\boldsymbol{p}} \cdot \boldsymbol{v}_{\text {in }}\left(\boldsymbol{p}_{0}\right) \geq \hat{\boldsymbol{p}} \cdot \boldsymbol{v}_{\text {out }}\left(\boldsymbol{p}_{0}\right)$.

- At an upper enabling threshold $\hat{\boldsymbol{p}} \cdot \boldsymbol{v}_{\text {in }}\left(\boldsymbol{p}_{0}\right) \leq \hat{\boldsymbol{p}} \cdot \boldsymbol{v}_{\text {out }}\left(\boldsymbol{p}_{0}\right)$.

- At a lower saturation threshold $\hat{\boldsymbol{p}} \cdot \boldsymbol{v}_{\text {in }}\left(\boldsymbol{p}_{0}\right) \leq \hat{\boldsymbol{p}} \cdot \boldsymbol{v}_{\text {out }}\left(\boldsymbol{p}_{0}\right)$.

- At an upper saturation threshold $\hat{\boldsymbol{p}} \cdot \boldsymbol{v}_{\text {in }}\left(\boldsymbol{p}_{0}\right) \geq \hat{\boldsymbol{p}} \cdot \boldsymbol{v}_{\text {out }}\left(\boldsymbol{p}_{0}\right)$.

- If $\hat{\boldsymbol{p}} \cdot \boldsymbol{v}_{\text {in }}\left(\boldsymbol{p}_{0}\right)=\hat{\boldsymbol{p}} \cdot \boldsymbol{v}_{\text {out }}\left(\boldsymbol{p}_{0}\right)$ then the curve $\mathcal{C}_{0}(\hat{\boldsymbol{p}})$ touches the kinematically allowed region tangentially. One should look at higher derivatives to determine the nature of the threshold. If the curve $\mathcal{C}_{0}(\hat{\boldsymbol{p}})$ touches the kinematically allowed region only at an isolated point, then we would hesitate to call this any kind of threshold. (These isolated points could nevertheless be interesting in their own right.) 
In the case of an isotropic energy-momentum relation this discussion simplifies. The momentum $\boldsymbol{p}$ is then parallel (or at worst anti-parallel) to the velocity $\boldsymbol{v}$ and so:

- At a lower enabling threshold $v_{\text {in }}\left(\boldsymbol{p}_{0}\right) \geq v_{\text {out }}\left(\boldsymbol{p}_{0}\right)$.

- At an upper enabling threshold $v_{\text {in }}\left(\boldsymbol{p}_{0}\right) \leq v_{\text {out }}\left(\boldsymbol{p}_{0}\right)$.

- At a lower saturation threshold $v_{\text {in }}\left(\boldsymbol{p}_{0}\right) \leq v_{\text {out }}\left(\boldsymbol{p}_{0}\right)$.

- At an upper saturation threshold $v_{\text {in }}\left(\boldsymbol{p}_{0}\right) \geq v_{\text {out }}\left(\boldsymbol{p}_{0}\right)$.

- The special case $v_{\text {in }}\left(\boldsymbol{p}_{0}\right)=v_{\text {out }}\left(\boldsymbol{p}_{0}\right)$ should be analyzed carefully by looking at higher derivatives. This might correspond to an "isolated point at which the decay is allowed"; we would then hesitate to call this any kind of threshold.

\subsubsection{Thresholds in terms of energy}

With some additional technical machinery we can rephrase the decay thresholds in terms of energy rather than 3-momentum. Some aspects of the analysis are more complicated, but we will now be able to deduce (at threshold) that $\boldsymbol{v}_{\text {in }}$ and $\boldsymbol{v}_{\text {out }}$ are parallel/anti-parallel. Let us now define

$$
\mathcal{P}_{\text {in }}\left(E_{0}\right)=\left\{\boldsymbol{p}_{0}: E_{0}\left(\boldsymbol{p}_{0}\right)=E_{0}\right\}
$$

which is the set of all possible total 3 -momenta given the input energy $E_{0}$. Now consider

$$
\mathcal{P}_{\text {out }}\left(\mathcal{P}_{\text {in }}\left(E_{0}\right)\right) \text {. }
$$

This is the set of planes (affine subspaces) of co-dimension 3 in $\mathbb{R}^{3 n}$ consisting of all possible output 3-momenta compatible with the specified input energy $E_{0}$. Then

$$
E_{\text {out }}\left(\mathcal{P}_{\text {out }}\left(\mathcal{P}_{\text {in }}\left(E_{0}\right)\right)\right)
$$

is the set of all possible 3-momentum-conserving output energies for input energy $E_{0}$. This will be a connected interval in $\mathbb{R}$. The decay process is then kinematically allowed if and only if

$$
E_{0} \in E_{\text {out }}\left(\mathcal{P}_{\text {out }}\left(\mathcal{P}_{\text {in }}\left(E_{0}\right)\right)\right)
$$

That is

$$
E_{\text {min }}\left(\mathcal{P}_{\text {out }}\left(\mathcal{P}_{\text {in }}\left(E_{0}\right)\right)\right) \leq E_{0} \leq E_{\text {max }}\left(\mathcal{P}_{\text {out }}\left(\mathcal{P}_{\text {in }}\left(E_{0}\right)\right)\right),
$$

where by this we mean

$$
\min _{\boldsymbol{p}_{0} \in \mathcal{P}_{\text {in }}\left(E_{0}\right)} E_{\min }\left(\boldsymbol{p}_{0}\right) \leq E_{0} \leq \max _{\boldsymbol{p}_{0} \in \mathcal{P}_{\text {in }}\left(E_{0}\right)} E_{\max }\left(\boldsymbol{p}_{0}\right) .
$$


Notice now that we are also extremizing over the 3 -momenta $\boldsymbol{p}_{0}$ compatible with the fixed initial energy $E_{0}$. Enabling thresholds will then occur at

$$
\min _{\boldsymbol{p}_{0} \in \mathcal{P}_{\mathrm{in}}\left(E_{0}\right)} E_{\min }\left(\boldsymbol{p}_{0}\right)=E_{0}
$$

and saturation thresholds at

$$
E_{0}=\max _{\boldsymbol{p}_{0} \in \mathcal{P}_{\text {in }}\left(E_{0}\right)} E_{\max }\left(\boldsymbol{p}_{0}\right)
$$

Either one of these thresholds can be characterized in terms of extrema of the related function

$$
\mathcal{E}\left(E_{0} ; \boldsymbol{p}_{i}, \boldsymbol{p}_{0}, \boldsymbol{\lambda}, \zeta\right)=\sum_{i=1}^{n} E_{i}\left(\boldsymbol{p}_{i}\right)-\boldsymbol{\lambda} \cdot\left[\sum_{i=1}^{n} \boldsymbol{p}_{i}-\boldsymbol{p}_{0}\right]-\zeta\left[E_{0}\left(\boldsymbol{p}_{0}\right)-E_{0}\right],
$$

where we now introduce two Lagrange multipliers, $\boldsymbol{\lambda}$ and $\zeta$, and we extremize over $\left(\boldsymbol{p}_{i}, \boldsymbol{p}_{0}, \boldsymbol{\lambda}, \zeta\right)$ while keeping $E_{0}$ fixed. Extremality with respect to the $\boldsymbol{p}_{i}$ yields

$$
\boldsymbol{v}_{i}=\boldsymbol{\lambda}=\boldsymbol{v}_{\text {out }},
$$

whereas extremality with respect to $\boldsymbol{p}_{0}$ yields

$$
\zeta v_{\text {in }}=\lambda .
$$

Since the sign and magnitude of $\zeta$ is unconstrained, this implies that at threshold the input velocity $\boldsymbol{v}_{\text {in }}$ is either parallel or anti-parallel to the common $\boldsymbol{v}_{\text {out }}$ of all the output particles:

$$
\zeta \boldsymbol{v}_{\text {in }}=\boldsymbol{v}_{\text {out }} .
$$

Note that we have gotten at least this far without assuming either spherical symmetry or any form of monotonicity.

\subsubsection{Asymmetric thresholds}

A particularly peculiar feature of Lorentz violating thresholds is the potential occurrence of asymmetric thresholds, where two identical decay particles might at threshold have unequal 3-momenta while traveling at the same 3 -velocity. (This phenomena was noted, in a more limited context, in reference [211].) The point is that while $\dot{\mathbf{x}}(\boldsymbol{p})$ is by assumption well defined, the inverse function $\boldsymbol{p}(\dot{\mathbf{x}})$ may be multi-valued. If this happens at threshold then two identical particles in the decay channel will have equal velocities but unequal momenta. Of course such multi-valued behaviour implies a multi-valued Lagrangian $L(\dot{\mathbf{x}})=\boldsymbol{p}(\dot{\mathbf{x}}) \cdot \dot{\mathbf{x}}-E(\boldsymbol{p}(\dot{\mathbf{x}}))$, which one 
may wish to exclude from any fundamental theory on physical grounds. (Such behaviour in an effective field theory is not particularly problematic.) To characterize when this can and cannot happen, note that local invertability of $\dot{\mathbf{x}}(\boldsymbol{p})$ requires the Jacobian matrix

$$
\frac{\partial \dot{\mathbf{x}}}{\partial \boldsymbol{p}}
$$

to be nonsingular. Equivalently the Hessian matrix

$$
\frac{\partial^{2} E}{\partial \boldsymbol{p} \partial \boldsymbol{p}}
$$

should be nonsingular.

Global invertability of $\dot{\mathbf{x}}(\boldsymbol{p})$ requires global non-singularity of the Hessian matrix. If we now add the extremely mild constraint that the Hessian matrix be positive definite at zero momentum (which is required to have any sensible Newtonian or Lorentzian limit at low momentum) then global invertability of $\dot{\mathbf{x}}(\boldsymbol{p})$ requires the Hessian matrix to be globally positive definite. But a globally positive definite Hessian matrix implies convexity of the energy-momentum relation $E(\boldsymbol{p})$.

Thus the existence (or not) of asymmetric thresholds is ultimately related to failures (or not) of the convexity of the energy-momentum relation $E(\boldsymbol{p})$. (For isotropic energy-momentum relations, this condition was phrased in terms of a positive curvature condition in reference [211].) This is why we can never get asymmetric thresholds in standard (non-tachyonic Lorentz invariant) special relativity, and why we do run the risk of asymmetric thresholds with (for example) lattice-type, tanhtype, and polynomial or rational polynomial energy-momentum relations. Thus the asymmetric threshold phenomena encountered by Mattingly, Jacobson, and Liberati in reference [211] is seen to have much wider applicability than the situations they considered.

\subsubsection{Some examples}

As an example of what can happen with asymmetric thresholds, it is quite possible for two identical particles to be emitted with almost all the momentum going into one particle, and almost none into the second particle. For instance if one takes two decay product particles obeying the tanh-type energy-momentum relation of (5.6), and shares the input momentum $p_{0}$ in the fractions $\left(\frac{1}{2} \pm \epsilon\right) p_{0}$, then the final state energy is

$$
E\left(p_{0}, \epsilon\right)=\sqrt{E_{0}^{2}+E_{*}^{2} \tanh \left(\frac{\left[\frac{1}{2}+\epsilon\right]^{2} p_{0}^{2} c^{2}}{E_{*}^{2}}\right)}+\sqrt{E_{0}^{2}+E_{*}^{2} \tanh \left(\frac{\left[\frac{1}{2}-\epsilon\right]^{2} p_{0}^{2} c^{2}}{E_{*}^{2}}\right)} .
$$


Depending on the precise ratios between $E_{0}, E_{*}$, and $p_{0}$, this can be minimized at $\epsilon=0$ or near $\epsilon=1 / 2$. See figure 5.6 .

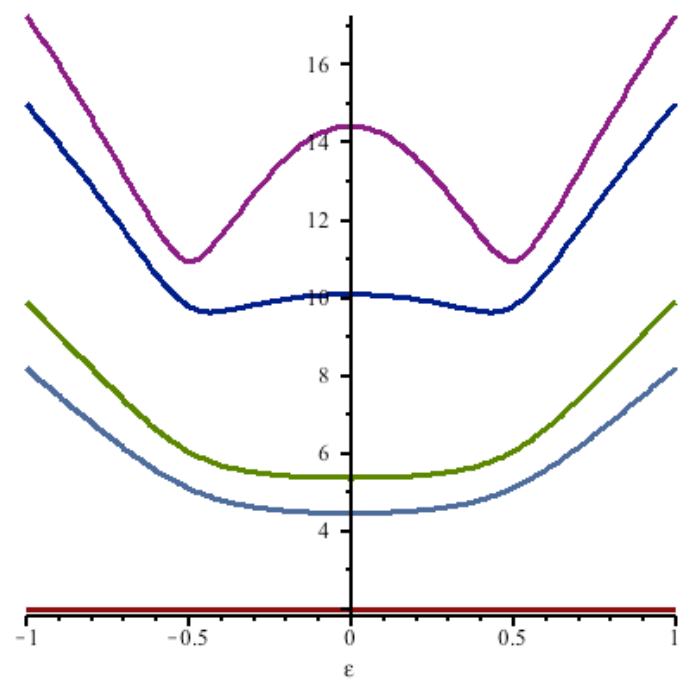

Figure 5.6: Energy as a function of $\epsilon$ for equation 5.60 holding $E_{0}$ and $E_{*}$ fixed and for five distinct values of $p_{0}$. Note how the location of the minimum (and hence the threshold) shifts from $\epsilon=0$ (a symmetric threshold) to $\epsilon \approx \pm 1 / 2$ (an asymmetric threshold). When the minimum occurs at $\epsilon \approx \pm 1 / 2$ almost all of the output momentum goes into one of the two identical particles, and almost none into the other.

Another highly nontrivial example, based roughly on equation (5.11), is to take

$$
E=\sqrt{E_{0}^{2}+c^{2}\left(\boldsymbol{p}_{x}^{2}+\boldsymbol{p}_{y}^{2}\right)+\frac{c^{2}}{3 p_{*}^{4}}\left\{\left(\boldsymbol{p}_{z}^{2}-p_{*}^{2}\right)^{3}-p_{*}^{6}\right\}} .
$$

This energy momentum relation is carefully chosen to be isotropic at low momentum, to have nice behaviour in the $x$ and $y$ directions, and to behave "interestingly" in the $z$ direction. Consider now a particle that moves in the $x$ direction so its original momentum is $\left(p_{0}, 0,0\right)$. Let it now decay into two identical particles of the type discussed above. Imposing 3-momentum conservation, the energy of the final state will be minimized when the final state particles have 3 -momenta $\left(p_{0} / 2,0, \pm p_{*}\right)$. So at threshold the initial state and two final state 3 -momenta are pointing in three different directions. The 3 -velocities of the two final state particles will however be equal, and will point along the $x$ axis.

These examples should be viewed as illustrations of the unusual phenomena that can occur once strict Lorentz invariance is violated. 


\subsubsection{Monotonicity}

What, if anything, can we say about monotonic energy-momentum relations? (This is a common but not universal simplifying assumption.) Consider a set of fixed directions $\hat{\boldsymbol{p}}_{i}$ and take

$$
E_{i}\left(\boldsymbol{p}_{i}\right)=E_{i}\left(p_{i} \hat{\boldsymbol{p}}_{i}\right)
$$

For each individual direction $\hat{\boldsymbol{p}}_{i}$ we can define monotonicity in terms of the magnitude $p_{i}$. Monotonicity means

$$
\frac{d E_{i}\left(p_{i} \hat{\boldsymbol{p}}_{i}\right)}{d p_{i}}>0
$$

but by the chain rule this implies

$$
\frac{d E_{i}\left(\boldsymbol{p}_{i}\right)}{d \boldsymbol{p}_{i}} \cdot \hat{\boldsymbol{p}}_{i}>0
$$

That is

$$
\boldsymbol{v}_{i}\left(\boldsymbol{p}_{i}\right) \cdot \boldsymbol{p}_{i}>0
$$

But then, by our previous arguments

$$
\frac{d E_{\min / \max }}{d \boldsymbol{p}_{0}} \cdot \boldsymbol{p}_{0}=\boldsymbol{v}_{\mathrm{out}} \cdot \boldsymbol{p}_{0}=\boldsymbol{v}_{\mathrm{out}} \cdot\left(\sum_{i=1}^{n} \boldsymbol{p}_{i}\right)=\sum_{i=1}^{n} \boldsymbol{v}_{\mathrm{out}} \cdot \boldsymbol{p}_{i}=\sum_{i} \boldsymbol{v}_{i} \cdot \boldsymbol{p}_{i}>0
$$

That is, as long as the individual $E_{i}\left(p_{i} \hat{\boldsymbol{p}}_{i}\right)$ are monotonic functions of the $p_{i}$, then $E_{\min / \max }\left(p_{0} \hat{\boldsymbol{p}}_{0}\right)$ is also monotonic as a function of $p_{0}$, and so the curves $\mathcal{C}_{ \pm}$bounding the kinematically allowed region will be monotonic. (Monotonicity of the boundary curves $\mathcal{C}_{ \pm}$can fail, and quite often will fail, if even one of the final state particles has a non-monotonic energy-momentum relation.)

\subsubsection{Isotropy}

If all the energy-momentum relations are isotropic (in the preferred (aether) frame) then

$$
\boldsymbol{v}_{i} \propto \boldsymbol{p}_{i} ; \quad \text { and } \quad \boldsymbol{v}_{\text {in }} \propto \boldsymbol{p}_{0} .
$$

So all the 3-momenta $\boldsymbol{p}_{i}$ and $\boldsymbol{p}_{0}$ are either parallel or anti-parallel to their corresponding 3 -velocities at threshold. Consequently, in view of the more general results deduced above,

$$
\boldsymbol{p}_{i} \propto \boldsymbol{p}_{0}
$$

That is, all 3-momenta are either parallel or anti-parallel to each other at threshold. 


\subsubsection{Monotonicity plus isotropy}

Only if we assume both isotropy and monotonicity can we deduce that the proportionality constants in the previous subsection are positive. In this case all 3-momenta and 3 -velocities are parallel at threshold. (This particular theorem was proven by Mattingly, Jacobson, and Liberati in [211.)

\subsection{Scattering thresholds}

Much of the previous discussion of decay thresholds carries over into the discussion of scattering thresholds, but there are just enough differences to make some separate discussion worthwhile.

\subsubsection{2-particle collisions}

Consider a 2-particle scattering process of the form

$$
X_{A}+X_{B} \rightarrow X_{1}+X_{2}+\ldots X_{n}
$$

involving particles of incoming momenta $\boldsymbol{p}_{A}$ and $\boldsymbol{p}_{B}$. Set $\boldsymbol{p}_{0}=\boldsymbol{p}_{A}+\boldsymbol{p}_{B}$. We can still define both $\mathcal{P}_{\text {out }}\left(\boldsymbol{p}_{0}\right)$ and $E_{\text {out }}\left(\mathcal{P}_{\text {out }}\left(\boldsymbol{p}_{0}\right)\right)$, and so construct both $E_{\min }\left(\boldsymbol{p}_{0}\right)$ and $E_{\max }\left(\boldsymbol{p}_{0}\right)$. The scattering is kinematically allowed if and only if

$$
E_{A}\left(\boldsymbol{p}_{A}\right)+E_{B}\left(\boldsymbol{p}_{B}\right) \in E_{\text {out }}\left(\mathcal{P}_{\text {out }}\left(\boldsymbol{p}_{A}+\boldsymbol{p}_{B}\right)\right) \text {. }
$$

That is, it is kinematically allowed if and only if

$$
E_{\min }\left(\boldsymbol{p}_{A}+\boldsymbol{p}_{B}\right) \leq E_{A}\left(\boldsymbol{p}_{A}\right)+E_{B}\left(\boldsymbol{p}_{B}\right) \leq E_{\max }\left(\boldsymbol{p}_{A}+\boldsymbol{p}_{B}\right) .
$$

Thresholds occur at the boundaries of these regions, that is, at:

$$
E_{\min }\left(\boldsymbol{p}_{A}+\boldsymbol{p}_{B}\right)=E_{A}\left(\boldsymbol{p}_{A}\right)+E_{B}\left(\boldsymbol{p}_{B}\right)
$$

and at

$$
E_{A}\left(\boldsymbol{p}_{A}\right)+E_{B}\left(\boldsymbol{p}_{B}\right)=E_{\max }\left(\boldsymbol{p}_{A}+\boldsymbol{p}_{B}\right) .
$$

To make further progress let us now define

$$
\mathcal{P}_{\text {in }}\left(E_{A}, E_{B}\right)=\left\{\boldsymbol{p}_{0}=\boldsymbol{p}_{A}+\boldsymbol{p}_{B}: E_{A}\left(\boldsymbol{p}_{A}\right)=E_{A}, E_{B}\left(\boldsymbol{p}_{B}\right)=E_{B}\right\},
$$

which is the set of all possible total input 3-momenta given the input energies $E_{A}$ and $E_{B}$. In terms of the notation 5.46 developed for decay processes we can write

$$
\mathcal{P}_{\text {in }}\left(E_{A}, E_{B}\right)=\mathcal{P}_{\text {in }}\left(E_{A}\right)+\mathcal{P}_{\text {in }}\left(E_{B}\right) .
$$


Now consider

$$
\mathcal{P}_{\text {out }}\left(\mathcal{P}_{\text {in }}\left(E_{A}, E_{B}\right)\right)
$$

This is the set of planes (affine subspaces) of co-dimension 3 in $\mathbb{R}^{3 n}$ consisting of all possible output 3-momenta compatible with the specified input energies $E_{A}$ and $E_{B}$. Then

$$
E_{\text {out }}\left(\mathcal{P}_{\text {out }}\left(\mathcal{P}_{\text {in }}\left(E_{A}, E_{B}\right)\right)\right)
$$

is the set of all possible 3-momentum-conserving output energies for input energies $E_{A}$ and $E_{B}$. This will be some connected interval in $\mathbb{R}$. The 2-particle scattering process is then kinematically allowed if and only if

$$
E_{A}+E_{B} \in E_{\text {out }}\left(\mathcal{P}_{\text {out }}\left(\mathcal{P}_{\text {in }}\left(E_{A}, E_{B}\right)\right)\right)
$$

That is

$$
E_{\min }\left(\mathcal{P}_{\text {out }}\left(\mathcal{P}_{\text {in }}\left(E_{A}, E_{B}\right)\right)\right) \leq E_{A}+E_{B} \leq E_{\max }\left(\mathcal{P}_{\text {out }}\left(\mathcal{P}_{\text {in }}\left(E_{A}, E_{B}\right)\right)\right),
$$

where by this we mean

$$
\min _{\boldsymbol{p}_{0} \in \mathcal{P}_{\text {in }}\left(E_{A}, E_{B}\right)} E_{\min }\left(\boldsymbol{p}_{0}\right) \leq E_{A}+E_{B} \leq \max _{\boldsymbol{p}_{0} \in \mathcal{P}_{\text {in }}\left(E_{A}, E_{B}\right)} E_{\max }\left(\boldsymbol{p}_{0}\right) .
$$

Thresholds will then occur at the edges of the kinematically allowed region.

Specifically, enabling thresholds will then occur at

$$
\min _{\boldsymbol{p}_{0} \in \mathcal{P}_{\mathrm{in}}\left(E_{A}, E_{B}\right)} E_{\min }\left(\boldsymbol{p}_{0}\right)=E_{A}+E_{B}
$$

and saturation thresholds at

$$
E_{A}+E_{B}=\max _{\boldsymbol{p}_{0} \in \mathcal{P}_{\text {in }}\left(E_{A}, E_{B}\right)} E_{\max }\left(\boldsymbol{p}_{0}\right) .
$$

Either one of these thresholds can be characterized in terms of extrema of the related function

$$
\mathcal{E}\left(E_{A}, E_{B} ; \boldsymbol{p}_{i}, \boldsymbol{p}_{A}, \boldsymbol{p}_{B}, \boldsymbol{\lambda}, \zeta_{A}, \zeta_{B}\right),
$$

where we now introduce three Lagrange multipliers, $\boldsymbol{\lambda}, \zeta_{A}$, and $\zeta_{B}$, and set

$$
\mathcal{E}=\sum_{i=1}^{n} E_{i}\left(\boldsymbol{p}_{i}\right)-\boldsymbol{\lambda} \cdot\left[\sum_{i=1}^{n} \boldsymbol{p}_{i}-\boldsymbol{p}_{A}-\boldsymbol{p}_{B}\right]-\zeta_{A}\left[E_{A}\left(\boldsymbol{p}_{A}\right)-E_{A}\right]-\zeta_{B}\left[E_{B}\left(\boldsymbol{p}_{B}\right)-E_{B}\right] .
$$

We now hold $\left(E_{A}, E_{B}\right)$ fixed, and extremize with respect to $\left(\boldsymbol{p}_{i}, \boldsymbol{p}_{A}, \boldsymbol{p}_{B}, \boldsymbol{\lambda}, \zeta_{A}, \zeta_{B}\right)$.

Extremality with respect to the $\boldsymbol{p}_{i}$ yields

$$
\boldsymbol{v}_{i}=\boldsymbol{\lambda}=\boldsymbol{v}_{\mathrm{out}},
$$


whereas extremality with respect to $\boldsymbol{p}_{A}$ and $\boldsymbol{p}_{B}$ yields

$$
\zeta_{A} \boldsymbol{v}_{A}=\boldsymbol{\lambda}=\zeta_{B} \boldsymbol{v}_{B}
$$

Since the signs (and magnitudes) of $\zeta_{A}$ and $\zeta_{B}$ are unconstrained, this implies that at threshold the two input velocities $\boldsymbol{v}_{A}$ and $\boldsymbol{v}_{B}$ are either parallel or anti-parallel, both to each other and to the common $\boldsymbol{v}_{\text {out }}$ of all the output particles:

$$
\zeta_{A} \boldsymbol{v}_{A}=-\boldsymbol{v}_{\mathrm{out}}=\zeta_{B} \boldsymbol{v}_{B}
$$

Note that we have gotten at least this far — parallel/anti-parallel input velocities without assuming either spherical symmetry or any form of monotonicity. Without additional assumptions we can go no further.

\subsubsection{Incoming 3-velocities}

In reference [211] Mattingly, Jacobson, and Liberati argue that assuming spherical symmetry and monotonicity of the energy-momentum relations the incoming 3-velocities must actually be anti-parallel at (enabling) threshold. Note that in the absence of exact Lorentz invariance spherical symmetry can at best only be expected to hold in the preferred (aether) fame.

Certainly if we assume spherical symmetry and monotonicity this result is now simple: Assuming spherical symmetry of the individual energy-momentum relations $E_{i}\left(\boldsymbol{p}_{i}\right)=E_{i}\left(p_{i}\right)$ for the decay products, we have $E_{\min }\left(\boldsymbol{p}_{0}\right)=E_{\min }\left(p_{0}\right)$. Furthermore assuming monotonicity of the individual $E_{i}\left(p_{i} \hat{\boldsymbol{p}}_{i}\right)$ we have already seen that this implies monotonicity of the $E_{\min }\left(p_{0} \hat{\boldsymbol{p}}\right)$. Then for enabling thresholds the quantity

$$
\min _{\boldsymbol{p}_{0} \in \mathcal{P}_{\text {in }}\left(E_{A}, E_{B}\right)} E_{\min }\left(\boldsymbol{p}_{0}\right)
$$

is minimized when $\left\|\boldsymbol{p}_{0}\right\|$ is minimized. Now assuming spherical symmetry for the incoming particles, so that $E_{A / B}\left(\boldsymbol{p}_{A / B}\right)=E_{A / B}\left(p_{A / B}\right)$, this occurs when when $\boldsymbol{p}_{A}$ and $\boldsymbol{p}_{B}$ are anti-parallel. Assuming spherical symmetry further implies $E(\boldsymbol{p})=$ $f\left(\frac{1}{2} p^{2}\right)$ so $\boldsymbol{v}=d E / d \boldsymbol{p}=f^{\prime}\left(\frac{1}{2} p^{2}\right) \boldsymbol{p}$. That is, $\boldsymbol{v}$ and $\boldsymbol{p}$ are either parallel or antiparallel. But monotonicity in turn implies $f^{\prime}\left(\frac{1}{2} p^{2}\right)>0$. That is, the individual $\boldsymbol{v}$ and $\boldsymbol{p}$ are parallel. This, in turn, implies the two incoming 3-velocities $\boldsymbol{v}_{A}$ and $\boldsymbol{v}_{B}$ are anti-parallel at enabling thresholds.

In contrast, for saturation thresholds we need to consider

$$
\max _{\boldsymbol{p}_{0} \in \mathcal{P}_{\text {in }}\left(E_{A}, E_{B}\right)} E_{\max }\left(\boldsymbol{p}_{0}\right) .
$$


Assuming spherical symmetry and monotonicity for the decay products this quantity is now maximized when $\left\|\boldsymbol{p}_{0}\right\|$ is maximized. But following the argument above, by assuming spherical symmetry and monotonicity for the input particles, this in turn implies that the incoming 3-momenta $\boldsymbol{p}_{A}$ and $\boldsymbol{p}_{B}$ are parallel at saturation thresholds, which in turn implies that the incoming 3-velocities $\boldsymbol{v}_{A}$ and $\boldsymbol{v}_{B}$ are parallel at saturation thresholds.

Both spherical symmetry and monotonicity are essential to these results. That is: Merely deducing that the incoming velocities are parallel/antiparallel at threshold is a generic result common to all Hamiltonian-based particle kinematics in a (Lorentz violating) homogeneous space-time. To go further and assert that incoming 3-velocities are anti-parallel at enabling thresholds, and parallel at saturation thresholds, requires the very much stronger assumptions of spherical symmetry and monotonicity. Our results are briefly summarized in tables 5.15 .3 .

\section{Summary of threshold behaviour: Final state particles}

\begin{tabular}{|l|c|c|c|}
\hline \hline & generic & isotropic & isotropic+monotonic \\
\hline \hline 3-velocities & equal & equal & equal \\
3 -momenta & uncorrelated & collinear & parallel \\
\hline \hline
\end{tabular}

Table 5.1: Behaviour at threshold for the 3-velocities and 3-momenta of outgoing final-state particles (compared to each other) under various assumptions.

\section{Summary of threshold behaviour: Decay — Initial state particle.}

\begin{tabular}{|c|c|c|c|}
\hline \hline & generic & isotropic & isotropic+monotonic \\
\hline \hline 3-velocity & collinear & collinear & collinear \\
3-momentum & uncorrelated & collinear & parallel \\
\hline \hline
\end{tabular}

Table 5.2: Behaviour at threshold for the 3-velocity and 3-momentum of the initial decaying particle (as compared to the final state decay product particles) under various assumptions. 
Summary of threshold behaviour: Scattering — Initial state particles.

\begin{tabular}{|c|c|c|c|}
\hline \hline & generic & isotropic & isotropic+monotonic \\
\hline \hline 3-velocities (enabling) & collinear & collinear & anti-parallel \\
3-momenta (enabling) & uncorrelated & collinear & anti-parallel \\
\hline \hline 3-velocities (saturation) & collinear & collinear & parallel \\
3-momenta (saturation) & uncorrelated & collinear & parallel \\
\hline \hline
\end{tabular}

Table 5.3: Behaviour at threshold for the 3-velocities and 3-momenta of the two initial state (incoming) particles (as compared to each other) under various assumptions.

\subsection{Conclusions regarding threshold theorems}

As we have seen, abandoning Lorentz invariance carries a very high price. The kinematically allowed region, and consequent threshold structure coming from the boundaries of the kinematically allowed region, for both decay processes and 2-particle (elastic or inelastic) scattering, is much more complicated than in the Lorentz invariant case. There are some limited number of truly general statements that one can make, but most of one's intuition has to be reassessed on a case by case basis. We have tried to carefully delineate exactly which assumptions are central to which results, concentrating on those results that depend only on the existence of a homogeneous space-time, and adding extra assumptions only when essential to obtaining specific specialised results.

The resulting framework is useful both in (an extremely wide class of) Lorentz violating extensions of the standard model of particle physics, and is also potentially of interest in quasi-particle settings where violations of Lorentz invariance (and even rotational invariance) are the norm rather than the exception.

Note that in the spirit of classical particle physics we have taken the Hamiltonian framework as being more fundamental, and the Lagrangian framework as derivative. As a side effect, the Lagrangians that typically arise for non-Lorentzcovariant free-particle Hamiltonians are often quite messy and unnatural. If one adopts the view that it is the Lagrangian framework that should be viewed as being more fundamental, this suggests that modified energy-momentum relations should be most naturally interpreted as effective phenomena rather than as fundamental physics. It is ultimately for this reason that one commonly focusses on perturbative deviations from Lorentz symmetry. A central theme of our discussion is that such 
a simplification is not always the most useful thing to do, and that there is merit to analysing thresholds in as general a setting as possible. One can surely consider that any serious attempt at phenomenological analysis of putative Lorentz violating observations will need to adopt a theoretical framework along the lines we have presented above. 


\section{Part II}

\section{Thermodynamics of space-time}





\section{Chapter 6}

\section{Thermodynamics of space-time An overview}

We have seen that, historically speaking, the quest for a quantum theory of gravitation has followed many different paths. One of the first approaches was the quantum geometrodynamics program, developed by Dirac, Bergmann, Arnowitt, Deser, Misner and others in the early sixties [299, 300, an approach that utilised the canonical quantisation procedure [189].

Other models have followed, such as for instance:

- The covariant approach [98, 101] where more field-theoretic techniques were used - the metric is split into a kinematical, fixed background part plus a dynamical perturbation;

- String theory - this was initially introduced to analyse the strong interactions from a novel angle. It was realised (after the great reinterpretation of the early 1980 ' $\bigsqcup^{1}$ ) that it automatically presented a spin-2 massless excitation, a graviton [60, 229, 230];

- The so-called connection-dynamics models, that include loop quantum gravity and spin foam models, and that follow the geometrodynamics program - that is, more from a relativistic point of view — but considering connections as the basic object [44, 50, 226, 242];

- Causal dynamical triangulation (CDT) [16, 17, 202 which can be seen as a modification of quantum Regge calculus [233];

\footnotetext{
${ }^{1}$ The presence of a spin-2 massless excitation was already discovered in 1974 by Scherk and Schwarz 252]; however, the full acceptance of this discovery by what became the modern string community occurred only a decade or so later.
} 
- Hor̆ava - Lifshitz gravity which is a traditional quantum field theory that breaks Lorentz invariance at ultra-high energies [156, 281, 297].

All these models are conceptually different and utilise different quantisation mechanisms - either the canonical or the covariant; however they present one common feature: The Einstein theory of general relativity is considered to be the starting point ${ }^{2}$. In other words the quantisation process is top-down, starting with a classical, well-established theory (the Einstein-Hilbert action) and the microscopic degrees of freedom are found consequently.

Other models instead establish some hypothetical fundamental, microscopic degrees of freedom and investigate the emergent properties when the system evolves, e.g. quantum graphity [178, 179, 236]. At the origin of the last approach there is the idea of induced gravity developed in 1967 by Andrei Sakharov [247, 277], in which space-time background emerges as some sort of mean field approximation of underlying microscopic degrees of freedom, related to what happens to Bose-Einstein condensate systems when one considers the fluid mechanics approximation.

In Sakharov's original formulation, general relativity arises as an emergent property from quantum field theory in roughly the same sense that hydrodynamics or continuum elasticity theory emerges from molecular physics [277].

The analogue models of space-time programme follows a similar line. One exploits the similarity between the equations describing the physics of some systems - Bose-Einstein condensates, pulses of light into filaments, and induced surface waves on a flume - and the curved space-time of general relativity, to gain some insight into the nature of the peculiar phenomena of GR [56, 197, 270, 298. In these models, the movement of the bulk (water, BEC, etc.) is approximated with a mean field theory, while the ripples propagate into an effective, emergent, curved metric.

The idea that gravitation may have a "thermodynamic" behaviour was initially raised by noticing the similarity between the equations describing some phenomena in condensed matter and general relativity. However it received more attention after the discovery of the proportionality of the area of a black hole horizon and its entropy [62], as well as the Hawking radiation [142]. Given these two results, the four laws of black holes mechanics and the four laws of thermodynamics were not just analogous but became identical. Therefore the question is "How does classical

\footnotetext{
${ }^{2}$ Technically speaking string theory does not consider general relativity as the starting point; however the string is at least defined within a special relativistic framework.
} 
general relativity know that horizon area would turn out to be a form of entropy, and that surface gravity is a temperature?".

In 1995 Ted Jacobson addressed this matter by reversing the usually followed logic, by trying to obtain the Einstein equations from thermodynamics equations [157] - thermostatic if one wants to be more precise. In particular, the author starts by assuming the validity of the area law, that is the proportionality between the entropy and the area of a causal horizon, and the Clausius relation đ $Q=T d S$ for the entropy, and works his way back to the Einstein equations by considering how the heat flux through a causal horizon would affect the space-time metric.

Even though some have argued that this derivation already implicitly contains the implications for the Einstein equation, hiding in the assumptions, the possibility to regard the Einstein equation as an equation of state is interesting and worth exploring - it could be an important starting point to shed some light into the microscopic nature of space-time.

For this reason, in chapter 7 we shall retrace some of the steps of Jacobson's argument, thoroughly analysing the definition of the thermodynamic system one wants to consider, firstly in the timelike setting and afterwards, by taking the limit to the null surface. For now our purpose is to generalise such argument to the case of generic bifurcation surfaces 3 while putting aside the full derivation of the Einstein equation.

In particular the goal is to give a proper definition of the Clausius entropy for bifurcate null surfaces when the bifurcation surface is no longer flat. At the end of the day what we shall obtain is a generalisation of the thermodynamics system used in Jacobson's argument.

We shall see that this more general construction sharply brings into focus some questions about the objective "reality" of entropy, or if it may be in some sense subjective and observer-dependent. These innocent questions open a Pandora's box of often inconclusive debate. A consensus opinion, though certainly not universally held, seems to be that Clausius entropy (thermodynamic entropy, defined via a Clausius relation $\mathrm{d} S=\mathrm{d} Q / T$ ) should be objectively real, but that the ontological status of statistical entropy (Shannon or von Neumann entropy) is much more ambiguous, and much more likely to be observer-dependent. This question is particularly pressing when it comes to understanding Bekenstein entropy (black hole entropy).

For this reason, and given the pivotal role that entropy plays in Jacobson's derivation of the Einstein equation, we think it is relevant to thoroughly study the

\footnotetext{
${ }^{3}$ For a definition of bifurcation surface refer to the footnote 1 , chapter 7 .
} 
mathematical characteristics of entropy, to which we shall dedicate chapter 8 . This way one can gain a deeper view into the matter of the equivalence among different definitions of entropy, a matter that is central to the solution of, for instance, the information loss paradox. Other attempts along these lines include [71, 90, 112, 168, 169, 232, 263, 268. 


\section{Chapter 7}

\section{Clausius entropy for arbitrary bifurcate null surfaces}

\subsection{Introduction}

Jacobson's thermodynamic derivation of the Einstein equations [157] has had, and continues to have, a profound influence on our understanding of the interface between thermodynamics and geometry. Jacobson's original construction associated heat fluxes and entropies only to local Rindler horizons [157], but left open the question as to whether some suitable notion of entropy could meaningfully be assigned to a broader class of null causal surfaces.

The construction we shall present in this chapter addresses this point, and is considerably more general than Jacobson's approach. We shall soon see that while the bifurcate nature of the local Rindler horizon is essential to the construction, other Rindler-specific features can easily be discarded. In particular, any null surface can be viewed as an observer-dependent causal boundary, a "virtual" causal boundary or virtual local horizon - and our construction can be viewed as providing a notion of virtual entropy for matter crossing arbitrary bifurcate virtual causal horizons.

Ultimately, we will argue that for arbitrary bifurcate null surfaces in curved space-time, at arbitrary cross-section $\mathcal{S}$ of the null surface, it is meaningful to define

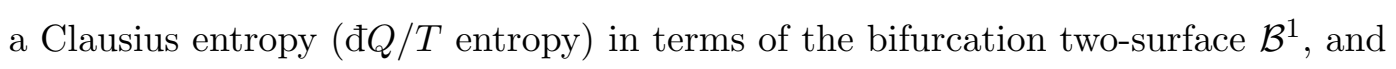
the affinely parameterised null generators. Since this is argued to hold for arbitrary

\footnotetext{
${ }^{1}$ A bifurcation surface is defined in relation to some Killing horizon. That is, if we consider a Killing vector $K$ and its associated Killing horizon $\mathcal{H}(p)$, a null hypersurface, with $p$ a point of the manifold $\mathcal{M}$, the bifurcation surface of $\mathcal{H}(p)$ is the set $\{K(p)=0\}$. Therefore it is a surface where the Killing vectors vanish. For a more detailed explanation the reader can refer to [61.
} 
"virtual" null surfaces, this can be viewed as thereby undermining the ontological reality of Clausius entropy; modulo some technical assumptions that we shall be very careful to make explicitly clear.

Indeed, the ontological status of entropy continues to generate much heated and inconclusive debate. Key questions are: Is entropy objectively "real"? Or is entropy in some sense subjective and observer-dependent? Part of the issue is that there are many different notions of entropy, and the extent to which they are universally equivalent is less than clear. At a minimum, one might wish to consider:

- Clausius entropy $(\mathrm{d} S=\mathrm{d} Q / T)$; often called thermodynamic entropy [86].

- Bekenstein entropy; black hole entropy [62].

- Statistical entropy; (Shannon [256, 257], von Neumann [289, 290], or entanglement entropy).

The extent to which these three notions can universally be identified is still a matter of debate, though in certain special cases they can be (and often are) degenerate.

Other related notions of entropy include Gibbs entropy, Boltzmann entropy, Srednicki entropy, Kolmogorov-Sinai entropy, and the Tsallis and Renyi entropies. Multiple attempts have been carried out in order to reconcile these different definitions, see for instance [71, 90, 112, 168, 169, 232, 263], and also to separate out and distinguish equilibrium and non-equilibrium notions of entropy [193]. (See also [85].) For additional general background see [43, 47, 78, 105, 106, 170, 235, 282, 294, 304, 305. The existence of all these definitions of entropy is what has inspired us to thoroughly studying its mathematical definition; these results will be presented in chapter 8 .

Typically, but not universally, the Clausius entropy is viewed as the most objectively real of these entropies. The Clausius entropy will be the central focus of this chapter, but even there the situation is extremely subtle. These ontological issues are central to Jacobson's "thermodynamic" derivation of the Einstein equations [157, where one part of the argument is based on an entanglement entropy interpretation of (a variant of) the Bekenstein entropy, and another part of the argument is based on (a variant of) the Clausius entropy applied to "local Rindler horizons". (These "local Rindler horizons" also arise in other situations such as those considered in [85] and $82 \sqrt[84]{3}$, and it may prove interesting to see to what extent those constructions could also be generalised.) We shall also see that a version of the generalised second law (GSL) 63 can be formulated for this virtual Clausius entropy, and can

\footnotetext{
${ }^{2}$ For the reader not familiar with the definition of the generalised second law, see appendix $\mathrm{C}$.
} 
be related to certain (nonstandard) integral variants of the null energy condition (NEC).

\section{Strategy}

Instead of addressing Jacobson's "thermodynamic" derivation directly we shall in the current chapter address a more modest goal: To what extent can a Clausiustype notion of entropy be associated with matter crossing arbitrary bifurcate null surfaces? We shall first work with exact Rindler horizons in flat Minkowski space, systematically and carefully extending the framework until we can successfully deal with arbitrary bifurcate null surfaces in curved space-time. This way we shall have a precise definition of the thermodynamic system we are dealing with.

Building on this construction, in future work we plan to more directly address the issue of the extent to which Bekenstein and Clausius entropies can universally be inter-related. Specifically: For which subset of causal horizons (virtual or otherwise) should they be inter-related? Under what situations should these concepts carefully be kept distinct?

\section{Jacobson's derivation of Einstein equation}

In this subsection we shall briefly summarise Jacobson's thermodynamic derivation of the Einstein equation. For a full description the reader can refer to [157]. Jacobson derived the Einstein equation by considering as a starting point the area law, the proportionality law of entropy and null surface (causal horizon) area, $d S=\eta$ đ $A-$ here $\mathrm{d} \mathcal{A}$ is related to $d \mathcal{A}$ that we shall use in the following - together with Clausius definition of the entropy $\mathrm{d} Q=T d S$, that connects heat, temperature and entropy, and it is defined for a system at the equilibrium. The thermodynamic system is defined through a local Rindler causal horizon, whose temperature is given through the Unruh effect - that we shall define in 7.2.1 — as seen by an accelerated observer just inside the horizon. Similarly, the heat đQ is interpreted as the energy flux seen by the same observer. The key idea is to demand that this picture/description holds for all the local Rindler causal horizons through each space-time point $p-$ therefore invoking the equivalence principle to view a small neighbourhood of each space-time point $p$ as a piece of flat space-time. In this way, the system is at the equilibrium and one can apply the relations introduced above - and that we shall describe in more details below. The geometric part is introduced into the picture by considering that the Clausius relation for the entropy, đQ $=T d S$, together with $d S=\eta$ đA, requires that the presence of the energy flux is associated with a focussing 
of the horizon generators, imposing a condition on the curvature of space-time. This will distort the causal structure of space-time in just such a way for the Einstein equation to hold, since a different entropy functional would produce field equations with a different dependance on the Ricci scalar and tensor. Viewed in this way, the Einstein equation is an equation of state.

\subsection{Flat Minkowski space-time}

To start the calculation is best to work in flat Minkowski space-time. That is, for now we are working in the framework of special relativity, not general relativity. This is not in contradiction with our purpose since, at this stage, we are not interested yet in finding how the heat-flux may affect the geometry of space-time.

\subsubsection{Heat flux, temperature, Clausius entropy}

In this section we shall introduce the thermodynamic quantities required to describe our system in terms of special relativistic concepts, such as timeline hypersurfaces, trajectories and observers.

Heat flux. To get a handle on the notion of heat flux đQ it is convenient to start with an infinitesimal segment of timelike hypersurface, (ruled by a congruence of future-pointing timelike vectors $V^{a}$, with outward spacelike normal $n^{a}$, and with hypersurface area element $d^{3} \Sigma$ ), and define a future-pointing flux vector

$$
F^{a}=-T^{a b} V_{b}
$$

It is then appropriate to define an infinitesimal heat flux $₫ Q$ by setting

$$
\mathrm{đ} Q=F^{a}\left(\mathrm{~d}^{3} \Sigma\right)_{a}=-T_{a b} V^{a} n^{b} \mathrm{~d}^{3} \Sigma .
$$

For finite segments of hypersurface we set

$$
\mathrm{d} Q=-\int T_{a b} V^{a} n^{b} \mathrm{~d}^{3} \Sigma .
$$

This is our version of Jacobson's equation (1), see reference [157], currently applied to timelike hypersurfaces. There would be universal agreement that this quantity defines the net energy flux across the segment of timelike hyper-surface, but perhaps less agreement that this energy flux can be equated with a heat flux. (For instance, some authors prefer to identify this quantity with $\mathrm{d} U$, the change in internal energy, while yet others might argue that this quantity should be identified with $\mathrm{d} H$, the 
change in enthalpy. For current purposes this subtlety is immaterial.) Following Jacobson [157, let us accept the above definition for the sake of argument and see where this identification leads. In particular one could assume that since in thermodynamics, heat is energy that flows between degrees of freedom that are not macroscopically observable, when considering space-time thermodynamics, one can interpret heat as energy that flows across a causal horizon. It can be felt via the gravitational field it generates, but its particular form or nature is unobservable from outside the causal horizon. Note that due to the $(-;+++)$ signature of space-time this is, perhaps counter-intuitively, the flux of energy in the direction of the normal $n^{a}$.

We shall now consider a sequence of timelike hypersurfaces, and construct an appropriate null limit. From the way this limit is set up it will soon be clear that we cannot deal with completely arbitrary null surfaces - the construction intrinsically is set up so that the null limit automatically yields bifurcate null surfaces. One of the advantages of Minkowski space is that it is possible to develop some exact results, many of which will even hold globally. We will subsequently invoke local flatness to extract more limited approximate results in curved space-times; approximate results which nevertheless hold up to an explicitly controlled level of accuracy in the vicinity of the bifurcation 2-surface. Ultimately we shall develop a construction valid in arbitrary curved space-times.

Temperature - Invoking the Unruh effect. A key physics step in the computation is to invoke the Unruh effect, that is acceleration radiation. In its original incarnation, this is a flat-space special-relativistic quantum-field-theory result whereby an accelerated observer will detect a thermal bath of quantum excitations with a temperature [271]:

$$
k_{B} T=\frac{\hbar a}{2 \pi},
$$

when an inertial observer, for which the QFT is in its usual Minkowski ground state, would observe none. This is due to the fact that the notion of vacuum depends on the trajectory of the observer through space-time [271].

Clausius entropy. We shall use the Unruh effect to define the differential Clausius entropy for the matter crossing any timelike hypersurface segment swept out by timelike observers of 4-acceleration $a$ by:

$$
\mathrm{d} S=\frac{\mathrm{d} Q}{T}=\frac{2 \pi k_{B}}{\hbar a} \mathrm{đ} Q=-\frac{2 \pi k_{B}}{\hbar a} T_{a b} V^{a} n^{b} \mathrm{~d}^{3} \Sigma .
$$


For a finite segment of hypersurface we could in principle allow the acceleration $a$ to vary from generator to generator of the timelike hypersurface, (for the time being the acceleration is to be kept constant along each generator, though later on we shall see how to relax this requirement), and would then have

$$
\mathrm{d} S=-\frac{2 \pi k_{B}}{\hbar} \int \frac{T_{a b}}{a} V^{a} n^{b} \mathrm{~d}^{3} \Sigma .
$$

In all explicit calculations below the hypersurfaces will be set up in such a manner that the acceleration $a$ is a constant over the hypersurface, so that

$$
\mathrm{d} S=-\frac{2 \pi k_{B}}{\hbar a} \int T_{a b} V^{a} n^{b} \mathrm{~d}^{3} \Sigma .
$$

A subtle and tricky point is that the $T_{a b}$ being used here is purely classical, whereas the Unruh temperature is associated with quantum fluctuations in the quantum ground state. (Jacobson refers to this as considering the "thermodynamic limit" [157.) In the presence of excitations above the quantum ground state it can be argued that the Unruh effect provides a lower bound on the physical temperature [1-4], and so an upper bound on $|\mathrm{đ} Q| / T$. Furthermore the $\mathrm{d} S$ defined above is a "virtual" quantity; there is no actual need for the timelike observers to be objectively real and physically present - the $\mathrm{d} S$ defined above is what would be seen by an imaginary swarm of timelike observers skimming along the timelike hypersurface.

In view of these issues, (identification of the heat flux, identification of the temperature, virtual status of the quantity $\mathrm{d} S$ ), some may refuse to call the quantity $\mathrm{d} S$ a Clausius entropy, and prefer to introduce yet another notion - perhaps "Jacobson entropy" might be appropriate? Be that as it may, provided one accepts this definition, and we hope the reader will agree this is a very plausible and physically interesting object to calculate, most of the technical computations of our derivation boil down to taking appropriate limits as the acceleration $a$ tends to infinity and the timelike surface becomes null.

\subsubsection{Rindler wedges}

We shall now begin to build our thermodynamical system. For this purpose we shall pick an arbitrary spacelike 2-plane in Minkowski space and choose coordinates so that this plane is

$$
x^{a}(x, y)=(0 ; x, y, 0) .
$$

Now add past and future light sheets, for convenience in the $+z$ direction. The resulting bifurcate null surface is

$$
x^{a}(t, x, y)=(t ; x, y,|t|) .
$$


The two null 3-d half-planes are joined by the spacelike bifurcation 2-plane at $t=0$. Now pick a sheet of hyperbolic timelike observers "close" to that null surface:

$$
x^{a}(\tau ; x, y)=\left(\frac{1}{a} \sinh (a \tau) ; x, y, \frac{1}{a} \cosh (a \tau)\right) .
$$

(Eventually we will want to take $a \rightarrow \infty$.) These observers have 4 -velocity

$$
V^{a}(\tau ; x, y)=(\cosh (a \tau) ; 0,0, \sinh (a \tau)) ; \quad\|V\|=1 ;
$$

and 4-acceleration

$$
A^{a}(\tau ; x, y)=a(\sinh (a \tau) ; 0,0, \cosh (a \tau)) ; \quad\|A\|=a ;
$$

while the hyperbolic timelike sheet they sweep out has 4-normal

$$
n^{a}(\tau ; x, y)=-(\sinh (a \tau) ; 0,0, \cosh (a \tau)) ; \quad\|n\|=1 .
$$

Here we have chosen the 4-normal to point towards the Rindler horizon; that is, away from the "observable" region containing the virtual timelike observers.

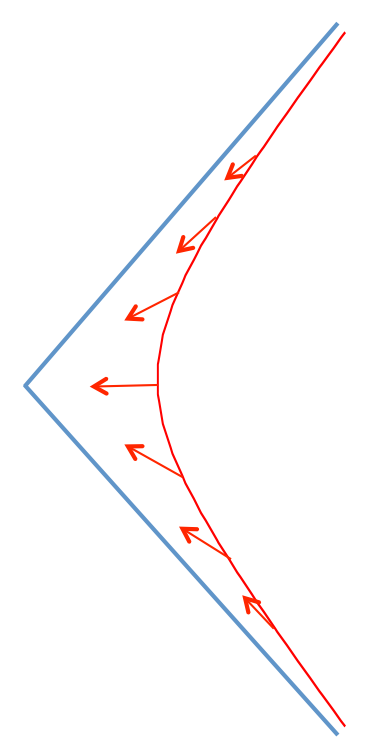

Figure 7.1: Rindler wedge with virtual hyperbolic timelike observer and 4-normals.

Note that the 4-normals point towards the Rindler horizon and asymptote to minus the 4-tangent at extremely late and extremely early proper times.

Note that on the time-like shell we have the restriction

$$
T_{a b}\left(x^{a}\right) \rightarrow T_{a b}(\tau, x, y)
$$


Then, setting $\mathrm{d}^{2} \mathcal{A}=\mathrm{d} x \mathrm{~d} y$ we have

$$
\mathrm{d} Q=-\int T_{a b} V^{a} n^{b} \mathrm{~d} \tau \mathrm{d}^{2} \mathcal{A}
$$

and so

$$
\frac{\mathrm{d} Q}{\mathrm{~d} \tau}=-\int T_{a b} V^{a} n^{b} \mathrm{~d}^{2} \mathcal{A}
$$

whence

$$
\frac{\mathrm{d} Q}{\mathrm{~d} t}=-\int T_{a b} V^{a} n^{b} \frac{\mathrm{d} \tau}{\mathrm{d} t} \mathrm{~d}^{2} \mathcal{A}
$$

With current conventions this is the flux of matter crossing the time-like shell in the direction of the Rindler horizon.

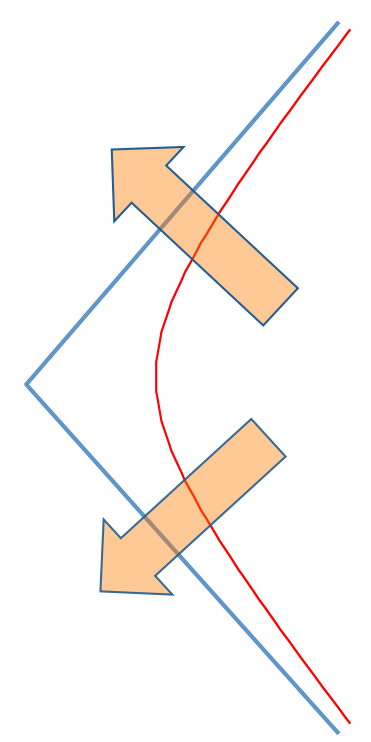

Figure 7.2: Formal direction of the heat flux $\mathrm{d} Q$.

Now compute (note the two minus signs cancel):

$$
\frac{\mathrm{d} Q}{\mathrm{~d} t}=\int\left\{\left[T_{00}+T_{33}\right] \sinh a \tau \cosh a \tau+T_{03}\left[\sinh ^{2} a \tau+\cosh ^{2} a \tau\right]\right\} \frac{\mathrm{d} \tau}{\mathrm{d} t} \mathrm{~d}^{2} \mathcal{A} .
$$

Substituting

$$
\sinh (a \tau)=a t ; \quad \cosh (a \tau)=\sqrt{1+(a t)^{2}}
$$

and

$$
\cosh (a \tau) \mathrm{d} \tau=\mathrm{d} t ; \quad \frac{\mathrm{d} \tau}{\mathrm{d} t}=\frac{1}{\sqrt{1+(a t)^{2}}}
$$


we see that we have

$$
\frac{\mathrm{d} Q}{\mathrm{~d} t}=\int\left\{\left[T_{00}+T_{33}\right] a t \sqrt{1+(a t)^{2}}+T_{03}\left[1+2(a t)^{2}\right]\right\} \frac{1}{\sqrt{1+(a t)^{2}}} \mathrm{~d}^{2} \mathcal{A},
$$

thereby implying

$$
\frac{\mathrm{d} Q}{\mathrm{~d} t}=a \int\left\{\left[T_{00}+T_{33}\right] t+T_{03}\left[\frac{1+2(a t)^{2}}{a \sqrt{1+(a t)^{2}}}\right]\right\} \mathrm{d}^{2} \mathcal{A} .
$$

If at this stage we let $a$ become large (this is mathematically somewhat illadvised, but close to Jacobson's original construction) then

$$
\begin{aligned}
\mathrm{d} Q / \mathrm{d} t & \rightarrow a \int\left\{\left[T_{00}+T_{33}\right] t+2 T_{03}|t|\right\} \mathrm{d}^{2} \mathcal{A}+\mathcal{O}(1 / a) \\
& =a t \int\left\{\left[T_{00}+T_{33}\right]+2 T_{03} \operatorname{sign}(t)\right\} \mathrm{d}^{2} \mathcal{A}+\mathcal{O}(1 / a)
\end{aligned}
$$

Note that the 2-d integral is to be evaluated on the transverse 2-plane (the $x-y$ plane) at time $t$. Now defining the null vectors

$$
k_{ \pm}^{a}=(1 ; 0,0, \operatorname{sign}(t)),
$$

which are the null normals on the two segments of the null surface, we have

$$
\mathrm{d} Q / \mathrm{d} t=a t \int\left\{T_{a b} k_{ \pm}^{a} k_{ \pm}^{b}\right\} \mathrm{d}^{2} \mathcal{A}+O(1 / a) .
$$

This is as close as we can get to a direct analogue of Jacobson's equation (2) as presented in reference [157]. (Note that because $a$ is merely large, not infinite, we are still dealing with timelike trajectories and timelike observers.)

It is mathematically safer to instead proceed in a slightly different manner as follows: Invoking the Unruh effect, relating the temperature $T$ to magnitude of the 4 -acceleration $a$, and explicitly using $k_{B} T=\hbar a /(2 \pi)$, we have

$$
\frac{\mathrm{d} Q / \mathrm{d} t}{T}=\frac{2 \pi k_{B}}{\hbar} \frac{\mathrm{d} Q / \mathrm{d} t}{a}=\frac{2 \pi k_{B}}{\hbar} \int\left\{\left[T_{00}+T_{33}\right] t+T_{03}\left[\frac{1+2(a t)^{2}}{a \sqrt{1+(a t)^{2}}}\right]\right\} \mathrm{d}^{2} \mathcal{A} .
$$

The key point is that it is this quantity that now has a completely well-defined limit as $a \rightarrow \infty$.

Indeed

$$
\begin{aligned}
\frac{\mathrm{d} Q / \mathrm{d} t}{T} & \rightarrow \frac{2 \pi k_{B}}{\hbar} \int\left\{\left[T_{00}+T_{33}\right] t+2 T_{03}|t|\right\} \mathrm{d}^{2} \mathcal{A} \\
& =\frac{2 \pi k_{B}}{\hbar} t \int\left\{\left[T_{00}+T_{33}\right]+2 T_{03} \operatorname{sign}(t)\right\} \mathrm{d}^{2} \mathcal{A}
\end{aligned}
$$


Therefore

$$
\frac{\mathrm{d} Q / \mathrm{d} t}{T} \rightarrow \frac{2 \pi k_{B}}{\hbar} t \int\left\{T_{a b} k_{ \pm}^{a} k_{ \pm}^{b}\right\} \mathrm{d}^{2} \mathcal{A} .
$$

This is a mathematically safer version of Jacobson's equation (2). It is important to realise this is an exact result, valid globally for all time. Note that both $\mathrm{d} Q$ and $T$ are diverging as $a \rightarrow \infty$, that is when approaching the null surface, while the ratio $\mathrm{d} S=\mathrm{d} Q / T$ remains finite. That is

$$
\frac{\mathrm{d} S}{\mathrm{~d} t}=\frac{2 \pi k_{B}}{\hbar} t \int\left\{T_{a b} k_{ \pm}^{a} k_{ \pm}^{b}\right\} \mathrm{d}^{2} \mathcal{A}
$$

Under normal circumstances the null energy condition [NEC $]^{3}$ is satisfied [57], then $\left\{T_{a b} k_{ \pm}^{a} k_{ \pm}^{b}\right\} \geq 0$ and the flux is inwards and positive for $t>0$. On the other hand, the inward flux is negative for $t<0$, indicating that it should be reinterpreted as a positive outward flux. That is: The NEC implies a variant of the GSL (generalized second law) holds for this version of Clausius entropy.

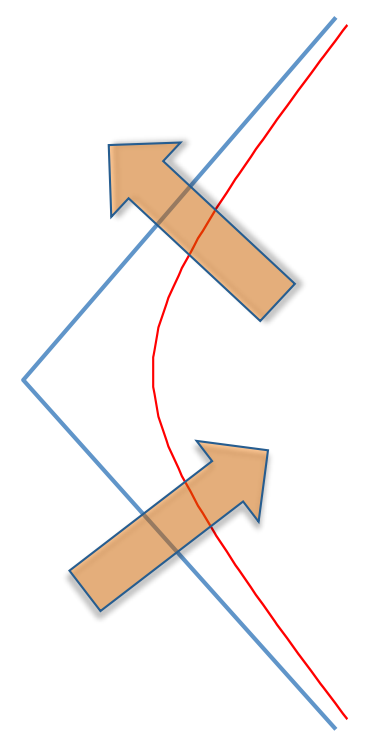

Figure 7.3: Physical direction of the heat flux đQ, (and the entropy flux $\mathrm{d} S$ ), assuming the GSL, (which is implied by the NEC), holds. Assuming the GSL, entropy can only emerge from the past null sheet and enter the future null sheet.

The only potentially naively unexpected part of this result is that it is explicitly linear in $t$. Technically that feature can ultimately be traced back to three facts:

\footnotetext{
${ }^{3}$ For the interested reader, more information about the energy conditions can be found in appendix B.
} 
1. That the location of the bifurcation 2-surface picks out a particular preferred origin for the time coordinate.

2. That symmetry enforces the flux to be zero at the bifurcation 2-surface.

3. That:

$$
\frac{1}{a} \frac{\mathrm{d} \tau}{\mathrm{d} t}=\frac{\sqrt{1+(a t)^{2}}}{a}=\sqrt{\frac{1}{a^{2}}+t^{2}} \rightarrow|t| .
$$

Note that the limiting procedure is utterly essential to get the explicit factor of $t$ above. Also, the use of the limiting procedure (starting from a timelike sheet) is needed for us to be able to invoke the Unruh effect - since the Unruh effect really makes sense only for timelike observers. We can now unambiguously write down Clausius entropy differences for arbitrary times (both positive or both negative) on the Rindler sheets:

$$
\Delta S_{\text {Clausius }}\left(t_{1}, t_{2}\right)=\frac{2 \pi k_{B}}{\hbar} \int_{t_{1}}^{t_{2}} \bar{t} \int T_{a b}(\bar{t}, x, y) k_{ \pm}^{a} k_{ \pm}^{b} \mathrm{~d}^{2} \mathcal{A} \mathrm{d} \bar{t} .
$$

Perhaps more tellingly we can (in Minkowski space) usefully define the Clausius entropy of the Rindler wedge at time $t$ as:

$$
S_{\text {Clausius }}(t)=S_{\mathcal{B}}+\frac{2 \pi k_{B}}{\hbar} \int_{0}^{t} \bar{t} \int T_{a b}(\bar{t}, x, y) k_{ \pm}^{a} k_{ \pm}^{b} \mathrm{~d}^{2} \mathcal{A} \mathrm{d} \bar{t}
$$

where $S_{\mathcal{B}}$ is the entropy to be associated with the bifurcation 2-plane itself, a quantity which is not constrained by the current argument. Again we emphasise that this variant of Jacobson's equation (2) is an exact result, valid globally for all time.

We shall now bootstrap this construction away from exact Rindler horizons in flat Minkowski space. We shall first deal with more complicated causal null surfaces in Minkowski space, and then extend the discussion to curved space-times.

\subsubsection{Causal null cones}

It is now easy to see that the construction above is not limited to Rindler wedges and flat null sheets. We are going to change the thermodynamic system by considering for instance causal null cones defined as follows: Choose a spacelike 2-sphere of radius $r_{0}$, with attached light cones expanding to both future and past. Adopt spherical polar coordinates so that the spacelike 2 -sphere is

$$
x^{a}(\theta, \phi)=\left(0 ; r_{0}, \theta, \phi\right),
$$

while the null surface is:

$$
x^{a}(t ; \theta, \phi)=\left(t ; r_{0}+|t|, \theta, \phi\right) .
$$


Now pick a spherical sheet of timelike observers

$$
x^{a}(\tau, \theta, \phi)=\left(\frac{1}{a} \sinh (a \tau) ; r_{0}+\frac{1}{a} \cosh (a \tau), \theta, \phi\right),
$$

now with 4-velocity

$$
V^{a}(\tau, \theta, \phi)=(\cosh (a \tau) ; \sinh (a \tau), 0,0) ; \quad\|V\|=1,
$$

and 4-acceleration

$$
A^{a}(\tau, \theta, \phi)=a(\sinh (a \tau) ; \cosh (a \tau), 0,0) ; \quad\|A\|=a,
$$

and 4-normal

$$
n^{a}(\tau, \theta, \phi)=-(\sinh (a \tau) ; \cosh (a \tau), 0,0) ; \quad\|n\|=1 .
$$

Then

$$
\mathrm{d} Q=-\int\left(r_{0}+\frac{1}{a} \cosh (a \tau)\right)^{2} T_{a b} V^{a} n^{b} \mathrm{~d} \tau \mathrm{d}^{2} \Omega .
$$

Note that this is an inwards entropy flux; towards the null cone.

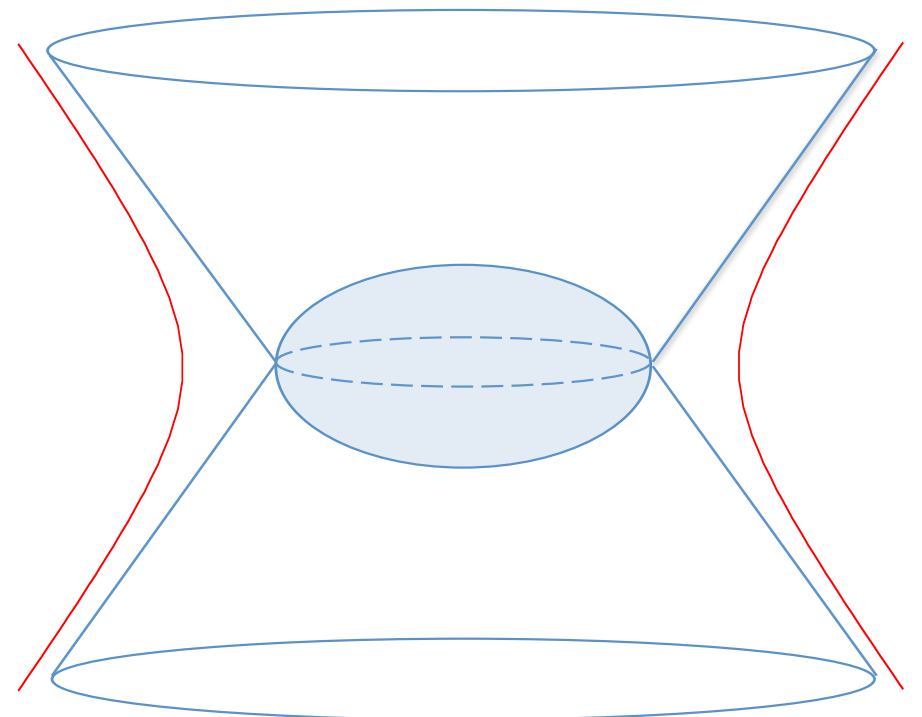

Figure 7.4: Bifurcate double null cone based on a spherical bifurcation 2-surface. Typical timelike observers indicated by red lines.

Now compute:

$$
\begin{aligned}
\frac{\mathrm{d} Q}{\mathrm{~d} t}= & \int\left(r_{0}+\frac{1}{a} \cosh (a \tau)\right)^{2} \\
& \times\left\{\left[T_{00}+T_{11}\right] \sinh a \tau \cosh a \tau+T_{01}\left[\sinh ^{2} a \tau+\cosh ^{2} a \tau\right]\right\} \frac{\mathrm{d} \tau}{\mathrm{d} t} \mathrm{~d}^{2} \Omega
\end{aligned}
$$


Strategically it is now best to substitute

$$
\sinh (a \tau)=a t ; \quad \cosh (a \tau)=\sqrt{1+(a t)^{2}},
$$

and

$$
\cosh (a \tau) \mathrm{d} \tau=\mathrm{d} t ; \quad \mathrm{d} \tau=\frac{\mathrm{d} t}{\sqrt{1+(a t)^{2}}}
$$

Then

$$
\begin{aligned}
\frac{\mathrm{d} Q}{\mathrm{~d} t}= & \int\left(r_{0}+\frac{\sqrt{1+(a t)^{2}}}{a}\right)^{2} \\
& \times\left\{\left[T_{00}+T_{11}\right] a t \sqrt{1+(a t)^{2}}+T_{01}\left[1+2(a t)^{2}\right]\right\} \frac{1}{\sqrt{1+(a t)^{2}}} \mathrm{~d}^{2} \Omega
\end{aligned}
$$

and so

$$
\frac{\mathrm{d} Q}{\mathrm{~d} t}=\int\left(r_{0}+\frac{\sqrt{1+(a t)^{2}}}{a}\right)^{2}\left\{\left[T_{00}+T_{11}\right] a t+T_{01} \frac{\left[1+2(a t)^{2}\right]}{\sqrt{1+(a t)^{2}}}\right\} \mathrm{d}^{2} \Omega .
$$

Therefore, again invoking the Unruh effect,

$$
\begin{aligned}
\frac{\mathrm{d} Q / \mathrm{d} t}{T} & =\frac{2 \pi k_{B}}{\hbar} \frac{\mathrm{d} Q / \mathrm{d} t}{a} \\
& =\frac{2 \pi k_{B}}{\hbar} \int\left(r_{0}+\frac{\sqrt{1+(a t)^{2}}}{a}\right)^{2}\left\{\left[T_{00}+T_{11}\right] t+T_{01} \frac{\left[1+2(a t)^{2}\right]}{a \sqrt{1+(a t)^{2}}}\right\} \mathrm{d}^{2} \Omega
\end{aligned}
$$

This quantity now has a well-defined limit as $a \rightarrow \infty$. Indeed we have the exact result

$$
\begin{aligned}
\frac{\mathrm{d} Q / \mathrm{d} t}{T} & \rightarrow \frac{2 \pi k_{B}}{\hbar} \int\left(r_{0}+|t|\right)^{2}\left\{\left[T_{00}+T_{11}\right] t+2 T_{01}|t|\right\} \mathrm{d}^{2} \Omega \\
& =\frac{2 \pi k_{B}}{\hbar}\left(r_{0}+|t|\right)^{2} t \int\left\{T_{a b} k_{ \pm}^{a} k_{ \pm}^{b}\right\} \mathrm{d}^{2} \Omega
\end{aligned}
$$

Here

$$
k_{ \pm}^{a}=(1 ; \operatorname{sign}(t), 0,0),
$$

and the angular integral is to be carried out over the 2-sphere at time $t$. Pulling the factor $\left(r_{0}+|t|\right)^{2}$ inside the integral we obtain the exact result

$$
\frac{\mathrm{d} Q / \mathrm{d} t}{T} \rightarrow \frac{\mathrm{d} S}{\mathrm{~d} t}=\frac{2 \pi k_{B}}{\hbar} t \int\left\{T_{a b} k_{ \pm}^{a} k_{ \pm}^{b}\right\} \mathrm{d}^{2} \mathcal{A}
$$

The integral is now over the area of the 2 -sphere at time $t$. Formally the final result is completely equivalent to that obtained for the Rindler wedge, even though 
various intermediate steps were somewhat different. This observation is particularly important, in that it will now allow us to greatly extend the range of validity of our previous result. In particular for any causal null cone (light cone) we now have

$$
S_{\text {Clausius }}(t)=S_{\mathcal{B}}+\frac{2 \pi k_{B}}{\hbar} \int_{0}^{t} \bar{t} \int T_{a b}(\bar{t}, \mathbf{x}(\theta, \phi, \bar{t})) k_{ \pm}^{a} k_{ \pm}^{b} \mathrm{~d}^{2} \mathcal{A} \mathrm{d} \bar{t}
$$

Here $S_{\mathcal{B}}$ is now the Clausius entropy to be associated with the bifurcation 2-sphere of radius $r_{0}$ located at $t=0$.

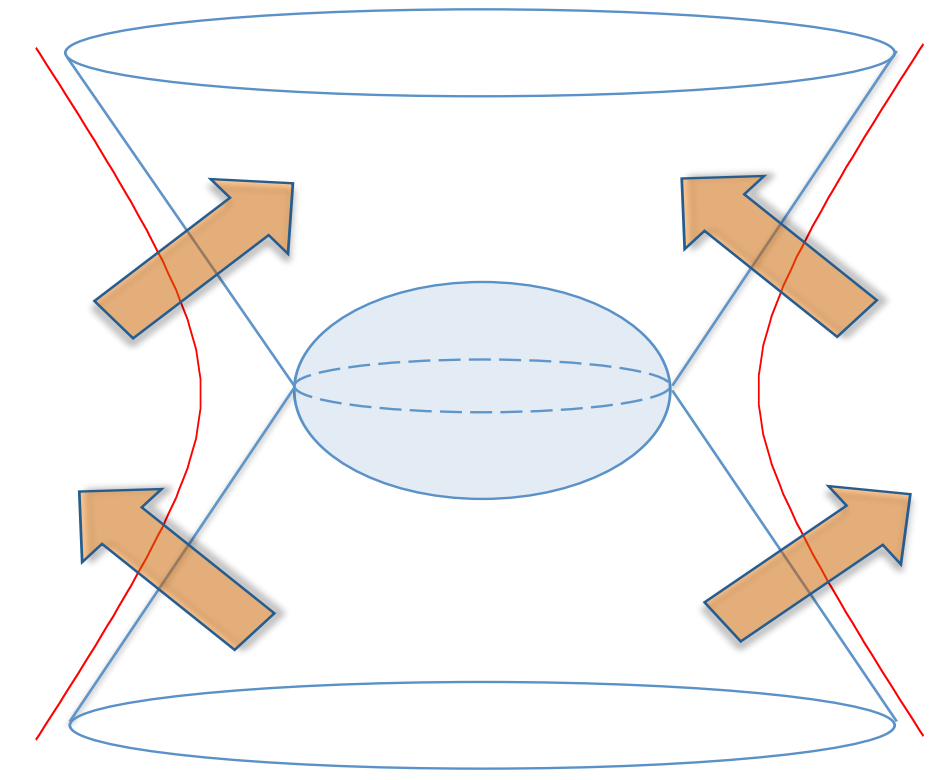

Figure 7.5: Direction of physical entropy fluxes, (assuming the GSL, which is implied by the NEC), for the bifurcate double null cone based on a spherical bifurcation 2-surface. Assuming the GSL, entropy can only emerge from the past null cone and enter the future null cone.

\subsubsection{Convex-base null conoids}

Consider now an arbitrary convex spacelike 2-surface. Choose Cartesian coordinates $x^{a}$ in Minkowski space, and generic coordinates $\xi^{i}$ on the 2 -surface. Then we can write

$$
x^{a}\left(\xi^{i}\right)=\left(0 ; \mathbf{x}\left(\xi^{i}\right)\right)
$$

Because the surface is convex, its outward pointing normals $\mathbf{n}(\xi)$ never intersect, so we can attach outward pointing past and future light rays to each point on the 
surface, and in turn these light rays will never intersect - so they define null surfaces. Then for these null sheets

$$
x^{a}\left(t ; \xi^{i}\right)=\left(t ; \mathbf{x}\left(\xi^{i}\right)+|t| \mathbf{n}\left(\xi^{i}\right)\right) .
$$

The resulting null conoids intersect at the original spacelike 2 -surface, which is therefore a bifurcation 2-surface. Along each one of these normal directions we can now simply repeat the calculation for causal null cones as presented above - which is why we put the effort into an explicit calculation for those simple cases. We again see

$$
\frac{\mathrm{d} Q / \mathrm{d} t}{T} \rightarrow \frac{\mathrm{d} S}{\mathrm{~d} t}=\frac{2 \pi k_{B}}{\hbar} t \int\left\{T_{a b} k_{ \pm}^{a} k_{ \pm}^{b}\right\} \mathrm{d}^{2} \mathcal{A}
$$

The integral is now over the cross-sectional area of the conoid at time $t$. The result is again exact and valid globally for all time. We now see

$$
S_{\text {Clausius }}(t)=S_{\mathcal{B}}+\frac{2 \pi k_{B}}{\hbar} \int_{0}^{t} \bar{t} \int T_{a b}(\bar{t}, \mathbf{x}(\xi, t)) k_{ \pm}^{a} k_{ \pm}^{b} \mathrm{~d}^{2} \mathcal{A} \mathrm{d} \bar{t}
$$

with the integral running over the null conoid.

\subsubsection{Causal diamonds}

To understand what happens if the bifurcation 2-surface is concave, (even partially concave), it is best to start with the highly-symmetric causal diamond configuration.

Choose a spacelike 2-sphere of radius $r_{0}$, but now with attached light cones contracting to both future and past. The null surface is now

$$
x^{a}(t ; \theta, \phi)=\left(t ; r_{0}-|t|, \theta, \phi\right) .
$$

The null curves generating the null surface now all collide at two points, at $t_{\text {collision }}=$ $\pm r_{0}$. A suitable class of timelike observers is now

$$
x^{a}(\tau, \theta, \phi)=\left(\frac{1}{a} \sinh (a \tau) ; r_{0}-\frac{1}{a} \cosh (a \tau), \theta, \phi\right),
$$

with the timelike observers colliding at

$$
\tau_{\text {collision }}= \pm \frac{1}{a} \cosh ^{-1}\left(r_{0} a\right)
$$

corresponding to

$$
t_{\text {collision }}= \pm \sqrt{r_{0}^{2}-\frac{1}{a^{2}}}
$$




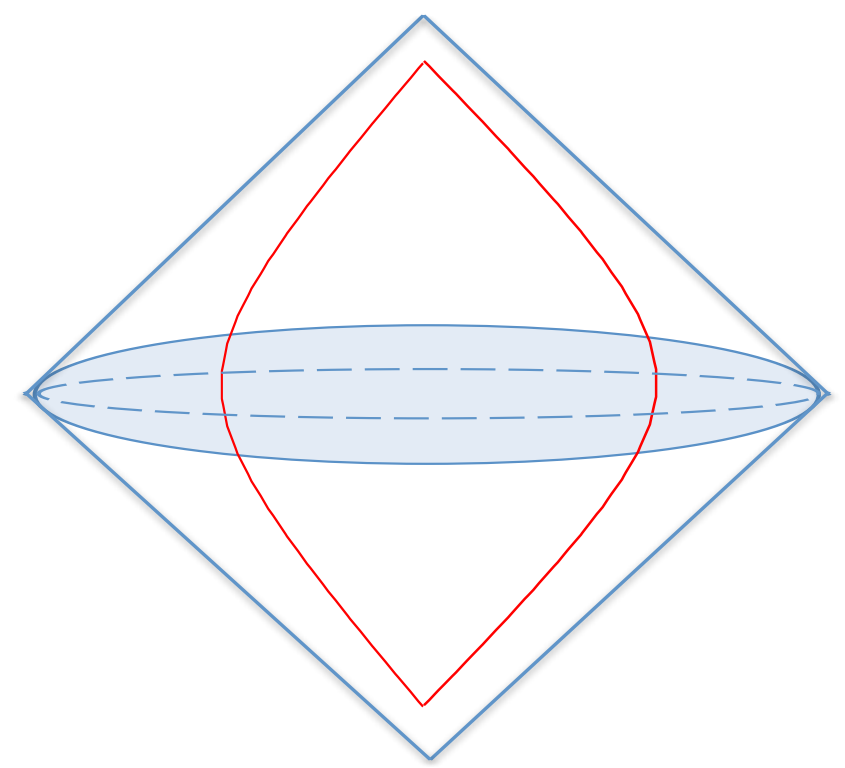

Figure 7.6: Causal diamond configuration. Typical timelike observers indicated by red lines. Note timelike observers now collide at finite time.

As long as we restrict attention to the finite interval where these timelike curves do not intersect then the previous computation for causal null cones can be carried over, and taking the appropriate limit we see that in the finite interval $t \in\left(-r_{0}, r_{0}\right)$ we still have

$$
\frac{\mathrm{d} Q / \mathrm{d} t}{T} \rightarrow \frac{\mathrm{d} S}{\mathrm{~d} t}=\frac{2 \pi k_{B}}{\hbar} t \int\left\{T_{a b} k_{ \pm}^{a} k_{ \pm}^{b}\right\} \mathrm{d}^{2} \mathcal{A} .
$$

The only minor quirk is that the timelike observers now reside inside the null surface, and that the timelike observers have 4-normal

$$
n^{a}(\tau, \theta, \phi)=(-\sinh (a \tau) ; \cosh (a \tau), 0,0) ; \quad\|n\|=1 .
$$

This implies one is now calculating an outward entropy flux. If one assumes the NEC this corresponds to a positive outwards flux for $t>0$ and a negative outwards (positive inwards) flux for $t<0$, which is compatible with the GSL. Again

$$
S_{\text {Clausius }}(t)=S_{\mathcal{B}}+\frac{2 \pi k_{B}}{\hbar} \int_{0}^{t} \bar{t} \int T_{a b}(\bar{t}, \mathbf{x}(\theta, \phi, \bar{t})) k_{ \pm}^{a} k_{ \pm}^{b} \mathrm{~d}^{2} \mathcal{A} \mathrm{d} \bar{t}
$$

Though historically Jacobson's construction was first applied to Rindler horizons, (and curved space local Rindler horizons), the causal diamond construction, (and its curved space analogue), can plausibly be argued to be more natural. In particular the causal diamond construction makes it clear that "local" causal horizons are

\footnotetext{
${ }^{4}$ We take this measure in order to keep the definition of the null surface clear.
} 
already sufficiently interesting - there is no need to continue the causal surfaces of interest all the way to (past or future) null infinity.

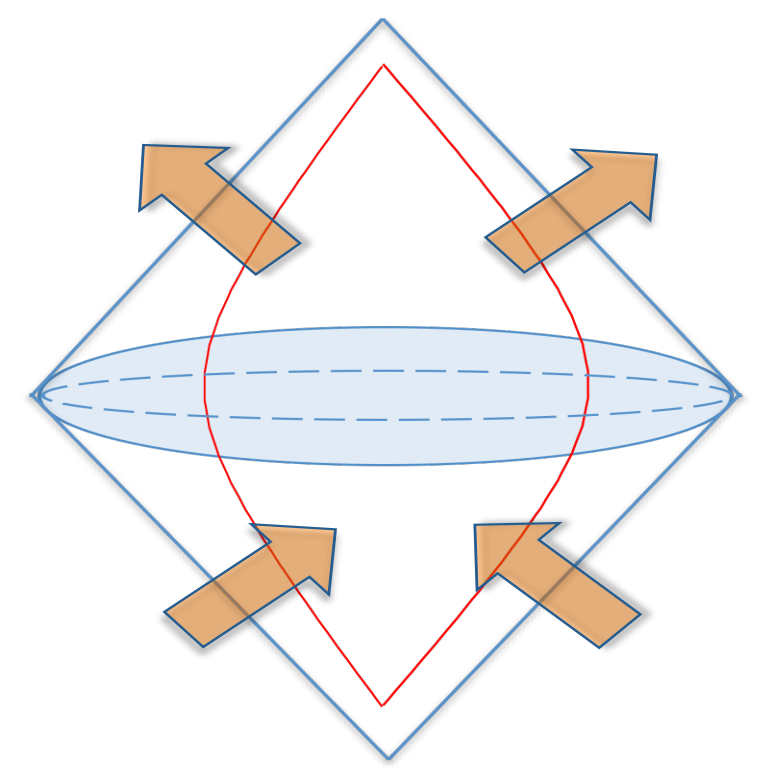

Figure 7.7: Direction of the physical entropy fluxes, (assuming the GSL, which is implied by the NEC), for the causal diamond configuration. Assuming the GSL, entropy can only enter the causal diamond from the past null cone and leave the causal diamond via the future null cone.

\subsubsection{Generic null conoids}

Now consider arbitrary null conoids in flat Minkowski space. We start with some arbitrary 2-surface at time $t=0$,

$$
x^{a}\left(\xi^{i}\right)=\left(0 ; \mathbf{x}\left(\xi^{i}\right)\right)
$$

but now with no constraint on the convexity of the 2 -surface. All the real work has already been done - the only obstruction comes from intersecting null normals. We see that over some finite interval $t \in\left(-t_{*}, t_{*}\right)$, where $t_{*}$ is determined by the time of first intersection of the null normals, we still have the exact result

$$
\frac{\mathrm{d} Q / \mathrm{d} t}{T} \rightarrow \frac{\mathrm{d} S}{\mathrm{~d} t}=\frac{2 \pi k_{B}}{\hbar} t \int\left\{T_{a b} k_{ \pm}^{a} k_{ \pm}^{b}\right\} \mathrm{d}^{2} \mathcal{A}
$$

Consequently, for the Clausius entropy, at as long as $t \in\left(-t_{*}, t_{*}\right)$, we still have the exact result

$$
S_{\text {Clausius }}(t)=S_{\mathcal{B}}+\frac{2 \pi k_{B}}{\hbar} \int_{0}^{t} \bar{t} \int T_{a b}(\bar{t}, \mathbf{x}(\xi, \bar{t})) k_{ \pm}^{a} k_{ \pm}^{b} \mathrm{~d}^{2} \mathcal{A} \mathrm{d} \bar{t}
$$


(Note again that we do not need to extrapolate the null causal surfaces all the way to past or future null infinity in order to have an interesting notion of Clausius entropy. Indeed, in the presence of null caustics, such an extension might, apart from being unnecessary, be outright impossible.) While the discussion started out with a straightforward computation for Rindler horizons, we have now bootstrapped it to a large class of bifurcate null surfaces - the only limitation at this stage is that there be an inertial frame in which the bifurcation surface can be chosen to lie on the hyperplane $t=0$.

\subsubsection{Generic bifurcate null surfaces}

As a penultimate step, we are now ready to address the situation for generic bifurcate null sheets in flat Minkowski space. We start with some completely arbitrary spacelike 2-surface,

$$
x^{a}\left(\xi^{i}\right)=\left(t_{0}\left(\xi^{i}\right) ; \mathbf{x}\left(\xi^{i}\right)\right),
$$

but now with no constraint on the convexity of the 2 -surface, nor with any constraint that the 2 -surface be contained in a hyperplane. Picking normals $\mathbf{n}(\xi)$ to the spatial part of this 2-surface, so we can attach outward pointing past and future light rays to each point on the surface - thereby defining null surfaces. Then for these null sheets one convenient parameterization is

$$
x^{a}\left(t ; \xi^{i}\right)=\left(t_{0}\left(\xi^{i}\right)+t ; \mathbf{x}\left(\xi^{i}\right)+|t| \mathbf{n}\left(\xi^{i}\right)\right)=x^{a}\left(\xi^{i}\right)+t k_{ \pm}^{a}\left(\xi^{i}\right) .
$$

Again, all the real work has already been done - the only significant obstruction comes from intersecting null normals. We see that over some finite interval $t \in$ $\left(-t_{*}, t_{*}\right)$, where $t_{*}$ is determined by the time of first intersection of the null normals, we still have the exact result

$$
\frac{\mathrm{d} Q / \mathrm{d} t}{T} \rightarrow \frac{\mathrm{d} S}{\mathrm{~d} t}=\frac{2 \pi k_{B}}{\hbar} t \int\left\{T_{a b}\left(x(\xi)+t k_{ \pm}(\xi)\right) k_{ \pm}^{a} k_{ \pm}^{b}\right\} \mathrm{d}^{2} \mathcal{A} .
$$

One subtlety is that this is not precisely $\mathrm{d} S / \mathrm{d} t$ "at physical time $t$ "; rather this $\mathrm{d} S / \mathrm{d} t$ is obtained by propagating the bifurcation surface $\mathcal{B}$ forward by time $t$ in some arbitrarily chosen rest frame and calculating the flux as a function of this evolution parameter. Consequently, for the Clausius entropy we can still write down an exact result

$$
S_{\text {Clausius }}(t)=S_{\mathcal{B}}+\frac{2 \pi k_{B}}{\hbar} \int_{0}^{t} \bar{t} \int T_{a b}\left(x(\xi)+\bar{t} k_{ \pm}(\xi)\right) k_{ \pm}^{a} k_{ \pm}^{b} \mathrm{~d}^{2} \mathcal{A} \mathrm{d} \bar{t} .
$$

As a final step we note that we could independently pick distinct affine parameters $\lambda$ on each null generator and write

$$
x^{a}\left(\lambda ; \xi^{i}\right)=x^{a}\left(\xi^{i}\right)+\lambda k_{ \pm}^{a}\left(\xi^{i}\right) ; \quad k_{ \pm}^{a}\left(\xi^{i}\right)=\frac{\mathrm{d} x^{a}\left(\lambda, \xi^{i}\right)}{\mathrm{d} \lambda} .
$$


Then taking $\mathcal{S}$ to be any spacelike cross-section of the bifurcate null surface we have

$$
S_{\text {Clausius }}(\mathcal{S})=S_{\mathcal{B}}+\frac{2 \pi k_{B}}{\hbar} \int_{\mathcal{B}}^{\mathcal{S}} \lambda T_{a b}\left(x(\xi)+\lambda k_{ \pm}(\xi)\right) k_{ \pm}^{a} k_{ \pm}^{b} \mathrm{~d}^{2} \mathcal{A} \mathrm{d} \lambda .
$$

Here the integral now runs over the entire null surface between $\mathcal{B}$ and $\mathcal{S}$. Note that the construction is manifestly independent of the way the affine parameter is normalised on each null generator.

We emphasize that while the discussion started out with a straightforward computation for exact Rindler horizons, we have now bootstrapped it to essentially arbitrary bifurcate null surfaces. We shall now perform a consistency check on the reasonableness of the construction, and then generalise the construction to curved space-times.

\subsection{Compatibility with the Bekenstein bound}

Before moving toward the next step, the case of curved space-time, we shall first check our proposal for the Clausius entropy for compatibility with the Bekenstein bound [64]:

$$
S \leq k_{B} \frac{2 \pi M R}{\hbar}
$$

This bound defines an upper limit on the entropy $\mathrm{S}$ that can be contained within a given finite region of space which has a finite amount of energy — or conversely, the maximum amount of information required to perfectly describe a given physical system down to the quantum level. This inequality was argued by Bekenstein to apply to weakly bound and weakly interacting systems. Since our Clausius notion of entropy is at this stage purely a Minkowski space result, the system is certainly weakly bound. But how are we to take this quantity,

$$
S_{\text {Clausius }}(t)=S_{\mathcal{B}}+\frac{2 \pi k_{B}}{\hbar} \int_{0}^{t} \bar{t} \int T_{a b}(\bar{t}, \mathbf{x}(\bar{t})) k_{ \pm}^{a} k_{ \pm}^{b} \mathrm{~d}^{2} \mathcal{A} \mathrm{d} \bar{t}
$$

and relate it to Bekenstein's bound? Certainly some extra assumptions will be required. (Such as, which bifurcate null surfaces will we consider?)

Let us first choose the bifurcation surface to be a single point, and the null surface to be its future light cone. When the bifurcation surface is a single point it is plausible to set $S_{\mathcal{B}} \rightarrow 0$. For simplicity, let us first take the stress-energy to be that of a spherically symmetric perfect fluid, then

$$
S_{\text {Clausius }}(t)=\frac{2 \pi k_{B}}{\hbar} 4 \pi \int_{0}^{t} \bar{t}^{3}(\rho+p) \mathrm{d} \bar{t} .
$$


Further note that $t \rightarrow R$, the radius of the light-sphere at time $t$. (We have set $c \rightarrow 1$.) For the specific case of a constant density fluid we then have

$$
S_{\text {Clausius }}(R)=\frac{2 \pi k_{B}}{\hbar} 4 \pi(\rho+p) \frac{R^{4}}{4} .
$$

But the Bekenstein bound is asserted to apply to weakly interacting systems, so it is for current purposes acceptable to take $p \in(0, \rho / 3)$. This is equivalent to the socalled "trace energy condition". The TEC is one of the oldest of the classical energy conditions, which was subsequently abandoned as fundamental physics, though it is certainly a useful characterization for weakly interacting matter [57]. Under these conditions $\rho+p<\frac{4}{3} \rho$, and so we have

$$
S_{\text {Clausius }}(R)<\frac{2 \pi k_{B}}{\hbar} \frac{4 \pi \rho R^{3}}{3} R=k_{B} \frac{2 \pi M R}{\hbar},
$$

as required. Consequently the notion of Clausius entropy defined in this chapter is indeed compatible with the Bekenstein bound. This gives us additional confidence that the construction developed above is physically interesting.

If the density and pressure are not constant (but are at least spherically symmetric) a minor variant of the above argument considers the quantity

$$
X=4 \pi \int_{0}^{R} r^{3}(\rho+p) \mathrm{d} r<4 \pi \int_{0}^{R} r^{3}(4 \rho / 3) \mathrm{d} r=\int_{0}^{R} r \mathrm{~d} m(r)+\frac{1}{3} \int_{0}^{R} r \mathrm{~d} m(r) .
$$

But then by integration by parts

$$
X<M R-\int_{0}^{R}\left[m(r) \mathrm{d} r-\frac{1}{3} r \mathrm{~d} m(r)\right]=M R+\frac{1}{3} \int_{0}^{R} r^{4} \frac{\mathrm{d}\left[m(r) / r^{3}\right]}{\mathrm{d} r} \mathrm{~d} r .
$$

If we now assume the average density is decreasing as one moves outwards, then $\mathrm{d}\left[m(r) / r^{3}\right] / \mathrm{d} r<0$, and the last term is negative. This falloff condition on the average density is one of the specific conditions Chandrasekhar uses in his investigations of non-relativistic stellar structure [81]. Then $X<M R$, and we again see that our construction for the Clausius entropy is at least compatible with Bekenstein's bound for weakly interacting systems.

\subsection{Curved space-time}

Now that we have carried out this exact calculation for flat Minkowski space, and checked for compatibility with wider notions of what we expect entropy to be, the generalisation to curved space-time is straightforward. 


\subsubsection{Near the bifurcation 2-surface}

First, consider an approximate calculation for curved space-time in the vicinity of the bifurcation 2-surface. Pick a bifurcate null surface in some curved space-time. Pick a point on that bifurcation 2 -surface. In the vicinity of that point adopt Riemann normal coordinates $x^{a}$, see figure 7.8 , so that

$$
g_{a b}=\eta_{a b}+\mathcal{O}\left(\left[x^{a}\right]^{2}\right) .
$$

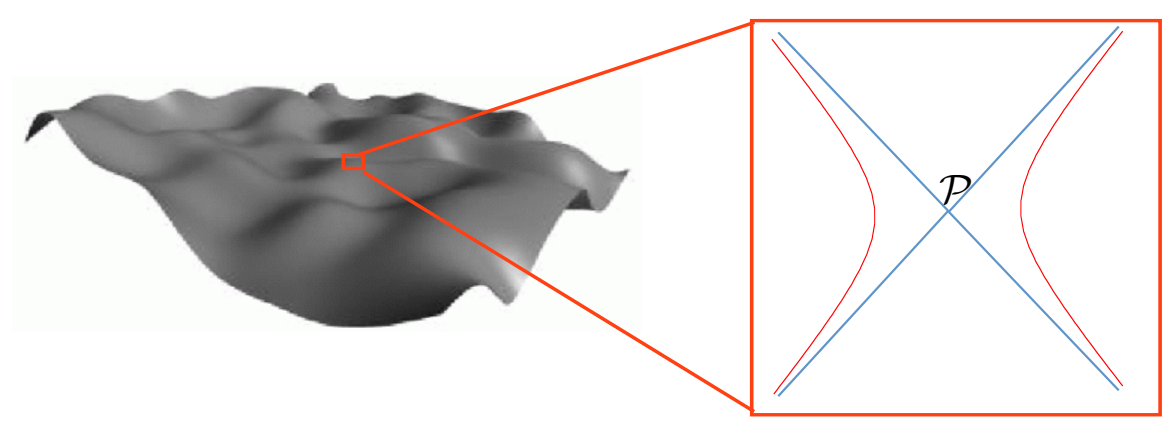

Figure 7.8: On a generic bifurcation surface in some curved space-time we pick a point $P$; in the vicinity of that point we adopt Riemann normal coordinates $x^{a}$, and the metric can be defined as $g_{a b}=\eta_{a b}+\mathcal{O}\left(\left[x^{a}\right]^{2}\right)$; here we have the special case of a Rindler horizon, with $\mathcal{P}$ representing the bifurcation 2-surface.

Then to the appropriate level of accuracy the null curves emanating from this point on the bifurcation 2-surface can be represented by

$$
x^{a}(t)=(t ; 0,0,|t|)+\mathcal{O}\left(t^{2}\right) .
$$

An appropriate timelike observer is

$$
x^{a}(\tau)=\left(\frac{1}{a} \sinh (a \tau) ; 0,0, \frac{1}{a} \cosh (a \tau)\right)+\mathcal{O}\left(\tau^{2}\right) .
$$

Equivalently

$$
x^{a}(t)=\left(t ; 0,0, \sqrt{t^{2}+\frac{1}{a^{2}}}\right)+\mathcal{O}\left(t^{2}\right) .
$$

Differentiating, the 4-velocity and 4-normal are determined up to terms of $\mathcal{O}(t)$, and the 4-acceleration up to terms of $\mathcal{O}(1)$. Furthermore, note that $T_{a b}(t)=T_{a b}(0)+$ $\mathcal{O}(t)$. 
There is a subtlety that one needs to take into account, that is the timelike observers are no longer exactly hyperbolic for all time. This would imply that the temperature of the system cannot be defined through the standard invocation of the Unruh effect. However there exists an adiabatic argument [51] demonstrating that the the Unruh effect will still hold adiabatically as long as the region over which the motion is close to hyperbolic, (the size of this region being determined by the space-time curvature), is large compared to the distance scale $1 / a$. We emphasise that there is now a considerable body of work on what might be called the "finite-time Unruh effect", wherein the original simplifying assumptions of eternal-constant-acceleration observers [271] is dispensed with. See for instance references [99, 187, 203, 218, 250, 253, 264]. (Similarly, in a black hole situation the existence of the Hawking effect is not dependent on the presence of an exact stationary [event] horizon, an approximate horizon satisfying a suitable adiabaticity condition is quite sufficient for the emission of a Planckian spectrum of Hawking photons [52 54, 278].)

Inserting all this into the previous computation, and taking the limit $a \rightarrow \infty$, we now get the approximate result

$$
\frac{\mathrm{d} Q / \mathrm{d} t}{T} \rightarrow \frac{\mathrm{d} S}{\mathrm{~d} t}=\frac{2 \pi k_{B}}{\hbar} t \int_{\mathcal{B}}\left\{T_{a b}(0) k_{ \pm}^{a} k_{ \pm}^{b}\right\} \mathrm{d}^{2} \mathcal{A}+\mathcal{O}\left(t^{2}\right)
$$

where to the relevant level of approximation the integral now runs over the bifurcation 2-surface $\mathcal{B}$. A subtlety here is that the Riemann normal coordinate construction implies that one is free to choose the $t$ coordinate independently on each null generator of the bifurcate null surface. This is equivalent to the ability to choose an arbitrary affine parameter $\lambda$ for each null generator, and to make this more explicit we can write

$$
\frac{\mathrm{d} Q / \mathrm{d} \lambda}{T} \rightarrow \frac{\mathrm{d} S}{\mathrm{~d} \lambda}=\frac{2 \pi k_{B}}{\hbar} \lambda \int_{\mathcal{B}}\left\{T_{a b}(0) k_{ \pm}^{a} k_{ \pm}^{b}\right\} \mathrm{d}^{2} \mathcal{A}+\mathcal{O}\left(\lambda^{2}\right) .
$$

If we restrict attention to "locally Rindler" bifurcate null surfaces then this expression is one of the key steps in Jacobson's thermodynamic derivation of the Einstein equations [157] - this is essentially Jacobson's equation (2) - but it is now clear from the present discussion that at least this aspect of Jacobson's argument is much more general, applying to essentially arbitrary bifurcate null surfaces. Note that this is the inward entropy flux. For positive $t$ and matter satisfying the NEC the flux is positive inwards. The sign flip for negative $t$ indicates the entropy flow is then positive outwards. Consequently, for the Clausius entropy we now have

$$
S_{\text {Clausius }}(t)=S_{\mathcal{B}}+\frac{2 \pi k_{B}}{\hbar} \frac{t^{2}}{2} \int_{\mathcal{B}}\left\{T_{a b}(0) k_{ \pm}^{a} k_{ \pm}^{b}\right\} \mathrm{d}^{2} \mathcal{A}+\mathcal{O}\left(t^{3}\right) .
$$


The constant term $S_{\mathcal{B}}$ is again undetermined by this argument. It is to be emphasized that this construction is to be applied to bifurcate null surfaces, not spacelike volumes, and the construction is both qualitatively and quantitatively different from entropy estimates built up by integrating up the thermodynamic entropy associated with small individual lumps of matter [1-4]. If we choose to work with arbitrary affine parameters and arbitrary spacelike sections $\mathcal{S}$ of the bifurcate null surface then we can rewrite the result as

$$
S_{\text {Clausius }}(\mathcal{S})=S_{\mathcal{B}}+\frac{2 \pi k_{B}}{\hbar} \int_{\mathcal{B}}\left\{\frac{\lambda^{2}}{2} T_{a b}(0) k_{ \pm}^{a} k_{ \pm}^{b}\right\} \mathrm{d}^{2} \mathcal{A}+\mathcal{O}\left(\lambda^{3}\right) .
$$

Here $\mathcal{S}$ is now the 2 -surface defined by propagating an affine distance $\lambda$ along each null generator emanating from the bifurcation 2 -surface $\mathcal{B}$.

\subsubsection{General formula for curved-space Clausius entropy}

In view of the above discussion we can now simply postulate that for arbitrary bifurcate null surfaces in curved space-time, at arbitrary cross-section $\mathcal{S}$ of the null surface

$$
S_{\text {Clausius }}(\mathcal{S}) \equiv S_{\mathcal{B}}+\frac{2 \pi k_{B}}{\hbar} \int_{\mathcal{B}}^{\mathcal{S}} \lambda T_{a b}(x(\xi, \lambda)) k_{ \pm}^{a} k_{ \pm}^{b} \mathrm{~d}^{2} \mathcal{A} \mathrm{d} \lambda .
$$

Note that $\lambda$ is an affine null parameter, that this integral is well-defined in the sense that it is invariant under rescaling of the affine null parameter, and that in view of the preceding discussion this construction passes all the consistency tests one might reasonably wish to impose. The only real restriction on the construction is that one should stop using it as soon as the null surface develops self-intersections.

\subsubsection{Generalized second law}

Note in particular that imposing the classical null energy condition - the NEC would guarantee positivity of the Clausius entropy flux, and imply a version of the GSL. Thus the NEC is a sufficient condition for the GSL to hold. (While there are certainly quantum-induced violations of the energy conditions [57], we would argue that they can be neglected in the thermodynamic limit.)

Note that a rather weaker sufficient condition for the GSL to hold, (for this definition of Clausius entropy), is that on all closed (or at worst edgeless) spacelike 2 -surfaces

$$
\int_{\mathcal{S}} T_{a b}(x(\xi)) k_{ \pm}^{a}(\xi) k_{ \pm}^{b}(\xi) \mathrm{d}^{2} \mathcal{A}(\xi) \geq 0 .
$$

A slightly different sufficient condition for the GSL to hold asymptotically, (at sufficiently late or early times, for this particular definition of Clausius entropy), is that 
on all future-pointing null half-geodesics we have

$$
\int_{0}^{\infty} \lambda T_{a b}(x(\lambda)) k_{+}^{a}(\lambda) k_{+}^{b}(\lambda) \mathrm{d} \lambda \geq 0
$$

and that on all past-pointing null half-geodesics we have

$$
\int_{-\infty}^{0} \lambda T_{a b}(x(\lambda)) k_{-}^{a}(\lambda) k_{-}^{b}(\lambda) \mathrm{d} \lambda \leq 0
$$

These conditions are certainly implied by the NEC, but are very much weaker than the NEC. Thus the GSL is seen to hold under very much weaker conditions than the NEC.

These integral variants of the NEC are also qualitatively very different from the standard averaged null energy condition (ANEC) $)^{5}$, see for instance [109, 113, 122, 275], or the Ford-Roman quantum inequalities [115, 117-119], or their variants [108, 110, 111, 116], or even the recent non-linear energy conditions explored in [207, 208. This strongly suggests these nonstandard integral variants of the NEC are well worth additional scrutiny.

\subsection{Conclusions regarding Clausius entropy}

The net result of this calculation, and the construction it inspires, is that one can associate an observer-dependent notion of entropy, very closely related to the Clausius entropy (thermodynamic entropy, đ $Q / T$ entropy) [86], and a generalization of Jacobson's local-Rindler entropy [157], to any arbitrary bifurcate null surface. That is, there is a certain sense in which even Clausius entropy (đ $Q / T$ entropy) is observer-dependent, with a "virtual Clausius entropy" being associated with arbitrary bifurcate "virtual causal horizons". (See also, for instance, the discussion in references [220 222].) This construction, because it generalizes one part of Jacobson's "thermodynamic" derivation of the Einstein equations, cuts to the heart of the issue of the putative universal equality of thermodynamic and entanglement entropy and into the matter of the very nature of the microscopic degrees of freedom of space-time.

\footnotetext{
${ }^{5}$ For a brief review regarding the average energy conditions see appendix $\mathrm{B}$.
} 


\section{Chapter 8}

\section{Infinite Shannon entropy}

We have seen that in the literature there exist several notions of entropy - that span from the thermodynamic, originally defined, Clausius entropy, to the statistical and quantum mechanical Shannon entropy and von Neumann entropy, to the information theoretic Rényi entropy and Tsallis entropy. Entropy seems to have an essential role in studying some of the phenomena of general relativity - see for example the equivalence between the four laws of thermodynamics and those for black holes and to understand the microscopic nature of space-time. However it is important to understand the degree of equivalence among all the definitions of the entropy utilised to solve these problems.

In this chapter we shall present our contribution to this effort by studying some of the characteristics of the Shannon (and some specific cases of von Neumann) entropy. In particular we shall consider the case of a probability distribution that is properly normalisable, and we shall demonstrate that its associated Shannon (or von Neumann) entropy can easily be infinite. Roughly speaking, this happens when arbitrarily small amounts of probability are dispersed into an infinite number of states; we shall quantify this observation and make it precise. Specifically, we shall see that large entropies cannot be localised in state space; large entropies can only be supported on an exponentially large number of states. We are, for the time being, interested in single-channel Shannon entropy in the information theoretic sense, not entropy in a stochastic field theory or QFT defined over some configuration space, on the grounds that this simple problem is a necessary precursor to understanding infinite entropy in a field theoretic context. 


\subsection{Introduction}

The classical Shannon entropy, (and closely related quantum von Neumann entropy), is a general purpose theoretical tool with a vast number of applications ranging from engineering to demographics to quantum information theory and other branches of theoretical physics [43, 235, 256, 257, 294, 305]. Stripped to its essentials, one considers a set of normalized probabilities $\sum_{n} p_{n}=1$, and analyzes the properties of the quantity:

$$
S=-\sum_{n} p_{n} \ln p_{n}
$$

Two major cases are of interest, when the index set $\{n\}$ characterizing the various "states" of the system is finite, and when it is countably infinite. A third case, when the index set $\{n\}$ is uncountably infinite, requires an integral formulation of the entropy, and we shall not presently have anything specific to say about this uncountable case. One way of justifying such a restriction is via an appeal to quantum mechanics where, in terms of a normalized density matrix $\operatorname{tr}[\rho]=1$, the von Neumann entropy is:

$$
S=-\operatorname{tr}[\rho \ln \rho] .
$$

If, (as is usual), quantum physics is formulated on a separable Hilbert space, then the density matrix can be diagonalized over a countable basis, and the von Neumann entropy reduces to the Shannon entropy over a countable (or possibly even finite) set of states. For this reason we shall restrict attention to the finite or countably infinite cases.

If the total number of states is finite, $N=\sum_{n} 1<\infty$, then by using the Jensen's inequality ${ }^{1}$ one obtains the upper bound:

$$
S \leq \ln N
$$

More subtly, even if the total number of states is infinite, $N=\sum_{n} 1=\infty$, then as long as the total number of states of non-zero probability is finite, $N^{\prime}=\sum_{n: p_{n}>0} 1<$ $\infty$, an equally elementary computation leads to the upper bound

$$
S \leq \ln N^{\prime} .
$$

These simple observations demonstrate that to obtain infinite Shannon entropy, an infinite number of states must have non-zero probability, in particular:

$$
N^{\prime} \geq \exp S
$$

\footnotetext{
${ }^{1}$ For a definition of the Jensen's inequality and its application to obtain inequality (8.3) the reader can refer to appendix $\mathrm{D}$
} 
But then, since the sum of the probabilities is unity, an infinite number of states must have non-zero but arbitrarily small probability. We shall now seek to quantify these observations in a straightforward and transparent manner. Some earlier rather technical work along these lines can be found in [259]. Our own interest in these issues was prompted by the more general issues raised in $62,171,78,105,106,157$, 263, 295, 296, 304 $]^{2}$. In the long run we would like to study infinite entropies that can arise in stochastic field theories and QFTs, but the single channel information theoretic context of the current chapter already provides some interesting subtleties.

\subsection{Some examples of infinite Shannon entropy}

To show that situations of infinite Shannon entropy can indeed occur perhaps the simplest example is to consider the sum:

$$
\Sigma(u)=\sum_{n=\lceil e\rceil}^{\infty} \frac{1}{n(\ln n)^{1+u}} ; \quad(\text { converges for } u>0 \text {, diverges for } u \leq 0) .
$$

Variants of this series are discussed for instance in Hardy [139, Hardy and Riesz [138, see esp p 5], and Shilov [258, see esp $\S 6.15 . c$ on p 192]. Here $\lceil x\rceil$ is the "ceiling" function, the smallest integer $\geq x$. The perhaps somewhat unexpected lower limit of summation $\lceil e\rceil$ is designed to ensure that $\ln n>0$ for all terms in the sum, so that one never has to raise a negative number to a real power. The corresponding probabilities (defined only for $n \geq\lceil e\rceil$ ) are

$$
p_{n}=\frac{1}{\Sigma(u) n(\ln n)^{1+u}} .
$$

These are well defined and properly normalised for $u>0$. But then

$$
\begin{aligned}
S & =\sum_{n} \frac{1}{\Sigma(u) n(\ln n)^{1+u}} \ln \left\{\Sigma(u) n(\ln n)^{1+u}\right\} \\
& =\ln \Sigma(u)+\frac{1}{\Sigma(u)} \sum_{n} \frac{1}{n(\ln n)^{u}}+\frac{1+u}{\Sigma(u)} \sum_{n} \frac{\ln \ln n}{n(\ln n)^{1+u}} \\
& =\ln \Sigma(u)+\frac{1}{\Sigma(u)} \sum_{n} \frac{1}{n(\ln n)^{u}}-\frac{\mathrm{d} \Sigma(u) / \mathrm{d} u}{\Sigma(u)} .
\end{aligned}
$$

The first and third terms converge for $u>0$, but the second term converges only for $u>1$. So this probability distribution is convergent but has infinite Shannon entropy

\footnotetext{
${ }^{2}$ In order to avoid the occurrence of infinite entropy, the QFT models that contemplate the existence of the area law for the entropy introduce a cut-off length. This more or less corresponds to introducing a probability gap, i.e. establishing that probabilities for certain states are zero. In the future we would like to explore the case in which such cut-off length is no longer present.
} 
over the entire range $u \in(0,1]$. The particular case $n=1$, where $p_{n} \propto \frac{1}{n(\ln n)^{2}}$ has previously been considered in a number of specific situations [65, 296]. Apart from the entire range $u \in(0,1]$ above, there are many more examples along similar lines. For instance one could consider the sums

$$
\begin{gathered}
\Sigma_{2}(u)=\sum_{n=\left\lceil e^{e}\right\rceil}^{\infty} \frac{1}{n \ln n(\ln \ln n)^{1+u}} ; \\
\Sigma_{3}(u)=\sum_{n=\left\lceil e^{e}\right\rceil}^{\infty} \frac{1}{n \ln n \ln \ln n(\ln \ln \ln n)^{1+u}} ;
\end{gathered}
$$

both of which converge for $u>0$ and diverge for $u \leq 0$, and the obvious infinite chain of generalizations thereof. Thereby (following the analysis above) one can easily construct an infinite chain of probability distributions that are convergent (and so are properly normalized) for $u>0$ but whose Shannon entropy converges only for $u>1$. These probability distributions are all convergent but have infinite Shannon entropy over the entire range $u \in(0,1]$. Even more baroque examples are possible, (but perhaps not desirable).

To briefly summarise, we have found a bound for the Shannon entropy in terms of the logarithm of the number of states with non-zero probability, therefore implying that infinite Shannon entropy occurs only with an infinite number of states with non-zero probability. Moreover, the condition that the sum of the probabilities is unity, dictates that an infinite number of states must have non-zero but arbitrary small probability. We shall now make these results more precise.

\subsection{Probability gap}

As a first step towards analyzing and quantifying the conditions under which infinite Shannon entropy can occur, let us define a notion of "probability gap" when there is a minimum non-zero probability. (The idea is to mimic the notion of "mass gap".) More precisely, let

$$
p_{*}=\inf \left\{p_{n}: p_{n}>0\right\} .
$$

If $p_{*}>0$ then, (since $1=\sum_{n} p_{n} \geq N^{\prime} p_{*}$ ), we have $N^{\prime} \leq 1 / p_{*} \leq \infty$. We see that only a finite number of the $p_{n}$ can then be non-zero, and in fact the infimum can be replaced by a minimum:

$$
p_{*}=\min \left\{p_{n}: p_{n}>0\right\} .
$$


So for all practical purposes the presence of a probability gap means the state space is effectively finite. Conversely if only a finite number of probabilities are non-zero then there is a probability gap. In particular it is elementary that

$$
S=-\sum_{n} p_{n} \ln p_{n} \leq-\sum_{n} p_{n} \ln p_{*}=-\ln p_{*}<\infty,
$$

though a slightly stronger result is also available

$$
S \leq \ln N^{\prime} \leq-\ln p_{*}<\infty .
$$

So we see very explicitly that for infinite Shannon entropy one cannot have a probability gap.

\subsection{Elementary bound leading to the Gibbs inequality}

Let us now try to be more quantitative. Based on the fact that for positive $x$ we have $[x \ln x]^{\prime \prime}=1 / x>0$, by using again Jensen's inequality ${ }^{3}$, we can state that for positive numbers

$$
x \ln (x / a)+y \ln (y / b) \geq(x+y) \ln \left(\frac{x+y}{a+b}\right),
$$

with equality only when $x / a=y / b$. See [140, p $97 \S 117]$.

Proof: Since the second derivative is positive

$$
\frac{a}{a+b} \tilde{x} \ln \tilde{x}+\frac{b}{a+b} \tilde{y} \ln \tilde{y} \geq \frac{a \tilde{x}+b \tilde{y}}{a+b} \ln \left(\frac{a \tilde{x}+b \tilde{y}}{a+b}\right),
$$

with equality only when $\tilde{x}=\tilde{y}$. Therefore

$$
a \tilde{x} \ln \tilde{x}+b \tilde{y} \ln \tilde{y} \geq(a \tilde{x}+b \tilde{y}) \ln \left(\frac{a \tilde{x}+b \tilde{y}}{a+b}\right) .
$$

Now rename $a \tilde{x} \rightarrow x$ and $b \tilde{y} \rightarrow y$ to obtain the desired result.

(It is worth explicitly verifying this since the justification is so elementary, and the payoff will be immediate.) Now iterate this result:

$$
x \ln (x / a)+y \ln (y / b)+z \ln (z / c) \geq(x+y+z) \ln \left(\frac{x+y+z}{a+b+c}\right) .
$$

More generally, for positive $x_{n}$ and $a_{n}$ :

$$
\sum_{n} x_{n} \ln \left(x_{n} / a_{n}\right) \geq\left(\sum_{n} x_{n}\right) \ln \left(\frac{\sum_{n} x_{n}}{\sum_{n} a_{n}}\right) .
$$

\footnotetext{
${ }^{3}$ See appendix $\mathrm{D}$ for an proof of the following inequality.
} 
When $\sum_{n} p_{n}=\sum_{n} q_{n}=1$ the above gives an elementary proof that

$$
\sum_{n} p_{n} \ln \left(p_{n} / q_{n}\right) \geq\left(\sum_{n} p_{n}\right) \ln \left(\frac{\sum_{n} p_{n}}{\sum q_{n}}\right)=0 .
$$

That is

$$
\sum_{n} p_{n} \ln p_{n} \geq \sum_{n} p_{n} \ln q_{n}
$$

so

$$
S \leq-\sum_{n} p_{n} \ln q_{n}
$$

This result is of course extremely well known, typically being referred to as the Gibbs inequality, (or the positivity theorem for relative entropy), with proofs most typically involving a less than elementary appeal to Jensen's inequality. We shall now apply this result to the matter at hand.

\subsection{Partial counts, partial probabilities, partial Shannon entropies}

Let us now consider the effect of summing only over some restricted subset $X$ of the total state space $\{n\}$. Define

$$
N_{X}=\sum_{n \in X} 1 ; \quad P_{X}=\sum_{n \in X} p_{n} ; \quad S_{X}=-\sum_{n \in X} p_{n} \ln p_{n}
$$

In particular, using the inequality demonstrated above, we have

$$
\sum_{n \in X} p_{n} \ln p_{n} \geq\left(\sum_{n \in X} p_{n}\right) \ln \left(\frac{\sum_{n \in X} p_{n}}{\sum_{n \in X} 1}\right)=P_{X} \ln \left(P_{X} / N_{X}\right) .
$$

Therefore

$$
S_{X} \leq P_{X}\left[\ln N_{X}-\ln P_{X}\right]
$$

Though this looks very similar to the entropy bound derived for the total entropy over a finite state space, there are significant differences - the current bound now tells you something deeper about the extent to which entropy can be localised in the state space. Indeed we can recast the bound as:

$$
N_{X} \geq P_{X} \exp \left(S_{X} / P_{X}\right)
$$

That is, packing a finite amount of entropy $S_{X}$ into a region containing total probability $P_{X}$ requires an exponentially large number of states $N_{X}$. 
Another way of interpreting this bound is to define the average probability per state, and average entropy per state, over the set $X$ by:

$$
\bar{P}_{X}=\frac{P_{X}}{N_{X}} ; \quad \bar{S}_{X}=\frac{S_{X}}{N_{X}} .
$$

Then

$$
\bar{S}_{X} \leq-\bar{P}_{X} \ln \bar{P}_{X}
$$

A slightly weaker but perhaps more intuitive bound is obtained if we vary the RHS of equation 8.27) with respect to $P_{X}$ while holding $N_{X}$ fixed (assume $N_{X} \geq\lceil e\rceil=3$ ). Then

$$
\frac{\partial \mathrm{RHS}}{\partial P_{X}}=\ln \left(N_{X} / P_{X}\right)-1>0 .
$$

So the maximum of the RHS occurs for $P_{X}=1$, and we see (for $N_{X} \geq 3$ )

$$
S_{X}<\ln N_{X}
$$

Similarly if we vary the RHS of equation 8.28 with respect to $P_{X}$ while holding $S_{X}$ fixed (assume $S_{X} \geq 1$ ). Then

$$
\frac{\partial \mathrm{RHS}}{\partial P_{X}}=\exp \left(S_{X} / P_{X}\right)\left\{1-S_{X} / P_{X}\right\}<0 .
$$

So the minimum of the RHS occurs for $P_{X}=1$, and we see (for $S_{X} \geq 1$ )

$$
N_{X}>\exp S_{X}
$$

The message to take from the logarithmic and exponential bounds is again that large Shannon entropies cannot be tightly localized in state space, large Shannon entropies must invariably come from exponentially large $\left(N_{X}>\exp S_{X}\right)$ regions of state space.

\subsection{Asymptotic estimates}

Let us now consider the effect of adding some extra order-based structure, by summing only over the high-probability sector of the total state space $\{n\}$. Define the quantities:

$$
N(p)=\sum_{n: p_{n} \geq p} 1 ; \quad P(p)=\sum_{n: p_{n} \geq p} p_{n} ; \quad S(p)=-\sum_{n: p_{n} \geq p} p_{n} \ln p_{n} .
$$

These are "probability cutoff" sums where the low probability events are excluded. Note

$$
\lim _{p \rightarrow 0} N(p) \rightarrow N ; \quad \lim _{p \rightarrow 0} P(p) \rightarrow 1 ; \quad \lim _{p \rightarrow 0} S(p) \rightarrow S
$$


where $N$ and $S$ may be infinite. It may sometimes be useful to define $N^{\prime}=\lim _{p \rightarrow 0^{+}} N(p)$ (which may again be infinite) in order to explicitly exclude the zero modes. Now

$$
S(p) \leq \sum_{n: p_{n} \geq p} p_{n}[-\ln p]=-P(p) \ln p \leq-\ln p .
$$

That is

$$
S(p) \leq-\ln p,
$$

so in some sense the total Shannon entropy can never be worse than "logarithmically divergent" in the probability cutoff. Similarly

$$
1 \geq P(p) \geq p N(p) ; \quad \text { that is } \quad N(p) \leq \frac{P(p)}{p} \leq \frac{1}{p} .
$$

We also have

$$
S(p) \leq P(p)[\ln N(p)-\ln P(p)] .
$$

Combining these results we regain

$$
S(p) \leq-P(p) \ln p \leq-\ln p .
$$

We again see that to get infinite Shannon entropy one needs an infinitely large number of arbitrarily low probability events.

\subsection{Entropy bounds from the Gibbs inequality}

Let us now obtain several explicit bounds directly from the Gibbs inequality. Consider the quantities $q_{n}=n^{-z} / \zeta(z)$ where $\zeta(z)$ is the Riemann zeta function. Then we have $\sum_{n=1}^{\infty} q_{n}=1$ for $z>1$. The Gibbs inequality becomes

$$
S \leq-\sum_{n=1}^{\infty} p_{n} \ln q_{n}=\ln \zeta(z)+z \sum_{n=1}^{\infty} p_{n} \ln n .
$$

Thus a sufficient condition for the Shannon entropy to be finite is

$$
\langle\ln n\rangle=\sum_{n=1}^{\infty} p_{n} \ln n<\infty .
$$

A number of quite similar results can easily be obtained:

- Consider for instance the quantity $\Sigma(\epsilon)=\sum_{n=1}^{\infty} \exp \left(-n^{\epsilon}\right)$. This sum is convergent when $\epsilon>0$. Then set $q_{n}=\exp \left(-n^{\epsilon}\right) / \Sigma(\epsilon)$, and note $\sum_{n=1}^{\infty} q_{n}=1$ provided $\epsilon>0$. Then the Gibbs inequality becomes

$$
S \leq-\sum_{n=1}^{\infty} p_{n} \ln q_{n}=\ln \Sigma(\epsilon)+\sum_{n=1}^{\infty} p_{n} n^{\epsilon} .
$$


Thus a sufficient condition for the Shannon entropy to be finite is that there exist some $\epsilon>0$ such that

$$
\left\langle n^{\epsilon}\right\rangle=\sum_{n=1}^{\infty} p_{n} n^{\epsilon}<\infty .
$$

This is of course part of a general phenomenon.

- Let $E_{n}$ be a collection of numbers such that $Z(\beta)=\sum_{n=1}^{\infty} \exp \left(-\beta E_{n}\right)$ converges for some at least one value of $\beta$. Now define $q_{n}=\exp \left(-\beta E_{n}\right) / Z(\beta)$, then $\sum_{n=1}^{\infty} q_{n}=1$ provided $\beta$ is such that the sum $Z(\beta)$ converges. Then the Gibbs inequality becomes

$$
S \leq-\sum_{n=1}^{\infty} p_{n} \ln q_{n}=\ln Z(\beta)+\beta \sum_{n=1}^{\infty} p_{n} E_{n} .
$$

Thus a sufficient condition for the Shannon entropy to be finite is that there exist some set of numbers $E_{n}$, and some $\beta$, such that the corresponding $Z(\beta)$ converges and such that

$$
\left\langle E_{n}\right\rangle=\sum_{n=1}^{\infty} p_{n} E_{n}<\infty
$$

On the other hand, deriving a necessary condition requires rather different tools. Let us first re-order (if necessary) the $p_{n}$ so they are in non-increasing order $\left(p_{n+1} \leq p_{n}\right)$. Then

$$
1 \geq \sum_{n=1}^{m} p_{n} \geq \sum_{n=1}^{m} p_{m}=m p_{m}
$$

That is, with this choice of ordering, we are guaranteed $p_{n} \leq 1 / n$. But then

$$
S=-\sum_{n=1}^{\infty} p_{n} \ln p_{n} \geq \sum_{n=1}^{\infty} p_{n} \ln n .
$$

Thus a necessary condition for the Shannon entropy to be finite, when the probabilities are sorted into non-increasing order, is that

$$
\langle\ln n\rangle=\sum_{n=1}^{\infty} p_{n} \ln n<\infty .
$$

We can eliminate the need for explicit re-ordering as follows: Using the previously defined object $N(p)=\sum_{n: p_{n} \geq p} 1$ we can define the quantities

$$
\tilde{p}_{n}=\max \{p: N(p) \geq n\} .
$$


Then $\tilde{p}_{n}$ is automatically a rearrangement of the $p_{n}$ in non-increasing order, and a necessary condition for the Shannon entropy to be finite is that defining

$$
\langle\ln n\rangle^{\sim} \equiv \sum_{n=1}^{\infty} \tilde{p}_{n} \ln n
$$

we have

$$
\langle\ln n\rangle^{\sim}<\infty .
$$

The mathematical tools used so far have been extremely basic inequalities and series; the analysis has been minimal. We shall now use some slightly more sophisticated analysis in the form of Dirichlet series.

\subsection{Dirichlet series}

Define the generalized zeta function, a particular type of Dirichlet series [138], by

$$
\zeta_{S}(z)=\sum_{n=1}^{\infty}\left(p_{n}\right)^{z} .
$$

One could think of the $S$ as standing either for Shannon or for entropy. A minor improvement is to explicitly exclude any states of zero probability and take

$$
\zeta_{S}(z)=\sum_{n}^{\prime}\left(p_{n}\right)^{z}=\sum_{n: p_{n}>0}\left(p_{n}\right)^{z} .
$$

By construction $\zeta_{S}(1)=\sum_{n=1}^{\infty} p_{n}=1$, so this particular Dirichlet series certainly converges (absolutely) for $z \geq 1$. The interesting question is whether it converges for any $z$ less than 1 . Note that

$$
S=-\left.\frac{\mathrm{d} \ln \zeta_{S}(z)}{\mathrm{d} z}\right|_{z=1} .
$$

If we now view $z$ as a complex number then, (in contrast to the usual situation for Taylor series where there is a radius of convergence), for Dirichlet series there is an abscissa of convergence $\sigma$ such that the series converges over the complex half-plane defined by 138

$$
\Re(z)>\sigma
$$

and diverges over the complex half plane defined by

$$
\Re(z)<\sigma .
$$


The line $\Re(z)=\sigma$ has to be treated delicately, in a manner somewhat analogous to the fact that that Taylor series behavior on the radius of convergence has to be treated delicately [138, see esp p 10]. The fact that the series is clearly convergent for real $z>1$ guarantees that $\sigma \leq 1$, the abscissa of convergence is bounded above by unity. The relevance of this observation lies in the following fact:

A sufficient condition for the entropy to be finite is that $\sigma<1$.

For a finite state space this is automatic. If we take the definition where zero probability states are excluded then the abscissa of convergence is $\sigma=-\infty$. (Even if we somewhat foolishly keep the zero probability states in the Dirichlet series, we still have $\sigma=0$.) For a countably infinite state space there is something to be proved. In particular, because all the coefficients in the generalized zeta function $\zeta_{S}(z)$ are positive, the real point on the abscissa of convergence is known to be a singular point of the function $\zeta_{S}(z)$. See [138, see p 10]. The word "singular" is used in the sense of "not analytic", so that there is no convergent Taylor series around the point $z=\sigma$. This happens if (for sufficiently large $m$ ) one of the derivatives diverges:

$$
\zeta_{S}^{(m)}(\sigma)=\sum_{n=1}^{\infty} p_{n}^{\sigma}\left(\ln p_{n}\right)^{m}=\infty .
$$

If this happens for $m=1$ (the first derivative) then the entropy is infinite. However, this might not happen until $m>1$, perhaps even much greater than 1 . That is:

Unfortunately $\sigma<1$ is not a necessary condition for finite entropy.

Example 1: As an explicit example of this phenomenon, recall that we had previously seen that the particular choice

$$
p_{n}=\frac{1}{\Sigma(u) n(\ln n)^{1+u}} ; \quad \Sigma(u)=\sum_{n=\lceil e\rceil}^{\infty} \frac{1}{n(\ln n)^{1+u}} ;
$$

leads to both finite entropy and normalizable probability for $u>1$. But the generalized zeta function corresponding to this particular $p_{n}$ is

$$
\zeta_{S}(z)=\Sigma(u)^{-z} \sum_{n=\lceil e\rceil}^{\infty} \frac{1}{n^{z}(\ln n)^{(1+u) z}} .
$$

And for this particular zeta function it is very easy to see that the abscissa of convergence is $\sigma=1$. (See for instance related discussion in Hardy [139], Hardy and Riesz [138], or Shilov [258]; the key point is that for $\Re(z) \neq 1$ the $n^{z}$ term dominates 
and controls convergence/divergence of the series. For $\Re(z)=1$ one has to look carefully at the exponent of the $\ln n$ term.) Furthermore, for this particular $p_{n}$ we see

$$
\zeta_{S}^{(m)}(1)=\sum_{n=\lceil e\rceil}^{\infty} p_{n}\left(\ln p_{n}\right)^{m}=\ln \Sigma(u)+\frac{1}{\Sigma(u)} \sum_{n=\lceil e\rceil}^{\infty} \frac{(\ln n+(1+u) \ln \ln n)^{m}}{n(\ln n)^{(1+u)}} .
$$

The dominant term in this last sum comes from the $(\ln n)^{m}$ in the numerator, so convergence of $\zeta_{S}^{(m)}(1)$ for the specific probability distribution presented in equation 8.62 is equivalent to convergence of

$$
\sum_{n=\lceil e\rceil}^{\infty} \frac{1}{n(\ln n)^{(1+u-m)}}
$$

But this series converges only for $u>m$. So even if the probabilities converge $(u>0)$, and even if in addition the entropy converges $(u>1)$, for any finite $u$ there will always be a sufficiently high derivative $(m>u)$ that fails to converge. This verifies by explicit example that $\sigma<1$ is not a necessary condition for finite entropy.

Example 2: On the other hand, let us now consider the following situation: Let $z_{0}>1$, and define quantities

$$
\tilde{p}_{n}=\frac{\left(p_{n}\right)^{z_{0}}}{\zeta_{S}\left(z_{0}\right)} .
$$

Then by construction $\sum_{n} \tilde{p}_{n}=1$ is absolutely convergent. The generalized zeta function associated with $\tilde{p}_{n}$ is

$$
\tilde{\zeta}_{S}(z)=\sum_{n}^{\prime}\left(\frac{\left(p_{n}\right)^{z_{0}}}{\zeta_{S}\left(z_{0}\right)}\right)^{z}=\frac{\zeta_{S}\left(z_{0} z\right)}{\zeta_{S}\left(z_{0}\right)^{z}} .
$$

But this implies $\tilde{\zeta}_{S}(z)$ is convergent for $z_{0} z \geq 1$, that is $z>1 / z_{0}$. Therefore the abscissa of convergence for the $\tilde{p}_{n}$ satisfies $\tilde{\sigma} \leq 1 / z_{0}<1$, which implies that the $\tilde{p}_{n}$ are guaranteed to have finite Shannon entropy. Now

$$
\ln \tilde{\zeta}_{S}(z)=\ln \zeta_{S}\left(z_{0} z\right)-z \ln \zeta_{S}\left(z_{0}\right) .
$$

A brief computation yields

$$
\tilde{S}=-\left.\frac{\mathrm{d} \tilde{\zeta}_{S}(z)}{\mathrm{d} z}\right|_{z=1}=-\left.z_{0} \frac{\mathrm{d} \ln \zeta_{S}(z)}{\mathrm{d} z}\right|_{z=z_{0}}+\ln \zeta_{S}\left(z_{0}\right) .
$$

This is certainly finite for any $z_{0}>1$. So it is extremely easy to construct a very large class of probability distributions which have both finite Shannon entropy and and an abscissa of convergence strictly less than unity: $\sigma<1$. 


\subsection{Discussion regarding infinite entropy}

To summarise the results, in this chapter we have considered situations of infinite entropy defined over a countably finite state space. Primarily we have focused on Shannon entropy, though the modifications required for dealing with von Neumann entropy are straightforward. We have considered single-channel information theoretic entropy, and the additional subtleties encountered in stochastic field theories and QFT are deferred for now. We have developed a number of very simple bounds and asymptotic estimates to probe the onset of infinite Shannon entropy, with an emphasis on keeping technical computations as simple as possible. Key results are that to obtain infinite Shannon entropy an infinite number of states must have nonzero but arbitrarily small probability, that the Shannon entropy can never be too divergent, and that in a suitable technical sense infinite Shannon entropy is never worse than logarithmic in the cutoff. The message to take from this logarithmic bound is that large Shannon entropies cannot be tightly localised in state space, large Shannon entropies must invariably come from exponentially large regions of the state space. 


\section{Part III}

\section{Gravitational effects on quantum physics}





\section{Chapter 9}

\section{Observing gravitational effects on quantum physics}

\subsection{Introduction}

In this last part of the thesis, we shall introduce an experimental proposal for testing the possible effects that motion - specifically acceleration - and curved space-time can have on quantum physics. The purpose of such testing is two-fold. On the one side it can be considered as an alternative approach to the quest for a quantum theory of/for gravitation - as we shall explain more in the following. On the other side, it raises interesting questions and gives some answers about implementing quantum technology for space-based communication, e.g. for satellites.

As we have seen in the introduction, chapter 1, the main problem with a quantum theory of gravitation is the lack of experimental evidence that could give some hints as to which direction to take 1 . The experiment we are about to describe would instead look at this problem from a different perspective, that is, investigating quantum physics and quantum effects at large scales. Indeed this promises to be experimentally achievable in the near future. For instance, in [237], the authors made several proposals of direct tests of quantum theory to larger and larger length scales - in some case approaching that of the radius of curvature of space-time, to probe the interaction between gravity and quantum phenomena - while in [251] the authors proposed to implement some quantum optics experiments in a groundto-space scenario using the International Space Station (ISS).

Indeed, we already have the technology for this kind of implementation, as

\footnotetext{
${ }^{1}$ This is due to the fact that the technology we have at our disposal does not allow us to probe energy regimes for which gravity has to be taken into account when analysing quantum processes.
} 
cutting-edge quantum experiments are reaching relativistic regimes, where the effects of gravity and motion on quantum properties can be experimentally tested. One major example is the remarkable result obtain in 2012 by the group led by A. Zeilinger in the Canary islands, where a teleportation protocol was successfully performed across $144 \mathrm{~km}$ between La Palma and Tenerife [272], by using entangled pairs of photons. With this experiment, the authors have shown that quantum entanglement can survive global distances, as predicted by quantum theory, and have therefore demonstrated the feasibility of quantum communication in space (for instance with the ISS).

This success, as well as related experimental developments [201, 213, 306], has convinced major space agencies, e.g. in Europe and Canada, to invest resources for the implementation of space-based quantum technologies [72, 274, 292], such as using satellites to distribute entanglement for quantum cryptography and teleportation (e.g. Space-QUEST project), and to install quantum clocks in space (e.g. Space Optical Clock project).

Given the importance that these experiments may have in the near future, it is crucial to address the matter of how relativistic effects will modify quantum entanglement - which lies at the base of those quantum phenomena such as quantum teleportation, quantum cryptography, etc. Indeed, already at the classical level, it is well-known that the Global Positioning System (GPS), a system of satellites used for time dissemination and navigation, requires both special and general relativistic corrections to determine time and positions accurately. Previous theoretical work has already addressed these fundamental questions by showing that gravity, motion, and space-time dynamics can create and degrade entanglement [14; and that acceleration produces observable effects on quantum teleportation [125]. However, current experimental space-based designs are yet to consider these findings.

\section{Quantum field theory arena}

Most proposals to implement quantum technologies in space have been developed within the framework of quantum mechanics where the effects of acceleration and gravity have been added ad-hoc. However the correct arena in which to look for relativistic effects is relativistic quantum field theory (QFT), which describes the behaviour of quantum fields in space-time. QFT is a semiclassical description in the sense that matter and radiation are quantised but the space-time is treated as a classical background - therefore there is no Einstein equation in the sense that space-time is not a dynamic quantity. However, unlike quantum mechanics, relativistic QFT naturally incorporates Lorentz invariance, as required by the postulates 
of relativity theory, and subsequently the concept of relativistic causality. Indeed, QFT successfully merges quantum theory and special relativity in the framework of the standard model of elementary particles. Moreover, QFT in curved space-time provides some partial answers to questions about the overlap of quantum mechanics and general relativity [70]. Very recently we have started to see some of its predictions be experimentally verified in [167, 244, 302] where the framework of analogue models of space-time has been exploited ${ }^{2}$. Therefore, in order to correctly account for effects that take place at increasing length and shorter time scales, quantum information techniques must be extended to a fully relativistic setting.

For this reason, in our proposal we have used a QFT framework to show that the gravitational field of the earth, and accelerated motion, can induce experimentally observable effects on the basic resource for quantum information and communication tasks - namely quantum entanglement. Furthermore, our research program aims, not only to characterise relativistic effects so that they can be corrected for, but also to learn how to exploit them in order to improve the performance of quantum technologies in space.

\subsection{The experimental proposal}

Recent work has shown that quantum entanglement between the field modes of localised systems, such as cavities, is sensitive to changes in acceleration [74]. Via the equivalence principle, this means that entanglement should be affected by changes in gravitational field potential, consequently affecting quantum communications between distant satellites. The purpose of our experiment is to demonstrate the effects of acceleration - and indirectly gravitation - by considering the entanglement between the excitations of two Bose-Einstein Condensates (BECs), each one of them prepared in a separate satellite. The BEC excitations we consider are known as quasiparticles or phonons that obey, under certain circumstances, a massless KleinGordon equation with a very slow speed of propagation [227. Low propagation speeds are the key element to enable the observation of the effect we describe below within realistic experimental regimes. We propose to entangle two BEC modes, one in each BEC, while the BECs move close to each other along the same circular earth orbit. One of the satellites will then undergo non-uniform motion to change

\footnotetext{
${ }^{2}$ As we shall see later on in this chapter, all three experiments have employed analogue systems, such as Bose-Einstein condensates, superconducting circuits or optic filaments, to build an effective curved space-time upon which some quantum field propagate, to test some QFT effects such as dynamical Casimir effect or Hawking radiation.
} 


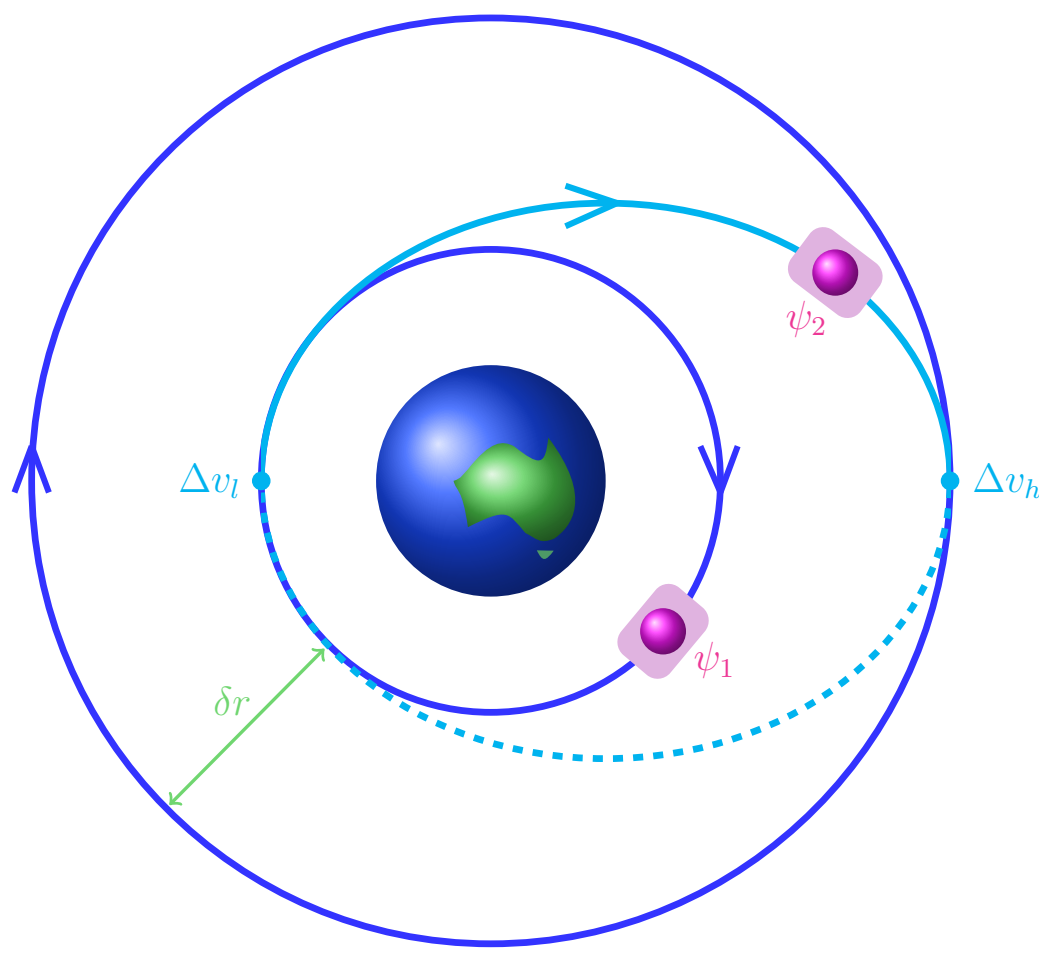

Figure 9.1: Experimental proposal. Two BECs inside separate satellites are entangled while both are in the same circular LEO orbit. Then one of them undergoes acceleration during a finite time in order to change to a different circular orbit, by means of a Hohmann transfer obit.

to an orbit subject to a different gravitational field strength, as shown in fig. 9.1 . Our analysis shows that the entanglement degradation between the BEC modes is a periodic function of the change in gravitational field strength in the orbit. This effect is significant already for typical parameters involved in microsatellite maneuvers, which is a great advantage since experiments involving such satellites have relatively low costs.

In the following part of chapter we shall give a description of all the pieces of the problem we would like to consider. For this reason we shall begin with a description of the Bose-Einstein condensate system we want to use as well as the motivation behind such choice. In the following parts we shall introduce some quantum-information notions, such as negativity and squeezing parameters. In the end, we shall describe the actual experimental set-up with the description, and motivation, of the satellites we want to use and the orbit changes we would like them to perform. 


\subsection{The Bose-Einstein condensate and acoustic metric}

We have explained above that the system we would like to consider for this experiment is composed of two Bose-Einstein condensates (BECs). There are mainly two reasons behind this choice and these are the very slow speed of propagation for the excitations - the speed of sound in BEC falls in the following interval $c_{s}=10^{-3}-10^{-2} \mathrm{~m} / \mathrm{s}$ - that should enhance the effects we want to see, and secondly the possibility, under certain conditions, to describe the system as an analogue model with an effective, emergent metric, upon which the excitations live. This second aspect is essential for the purpose of our experiment as we shall explain in this section.

In the absence of atomic collisions, a BEC can in principle reach absolute zero temperature and be described by a classical mean field. However, collisions are always present and therefore, in the superfluid regime, the condensate is better described by a mean field classical background plus quantum fluctuations. The fluctuations, for length scales larger than the so-called healing length $\mathrm{s}^{3}$, behave like a phononic quantum field.

If the BEC system is homogenous, irrotational and inviscid one can consider the analogue model approximation for which the classical background energy density, pressure and number density play the role of an effective space-time metric which in principle can be curved. We present the details from which this approximation arises in appendix E. The phononic field $\Pi(\xi)$ can be expanded in terms of the Bogoliubov modes $\phi(\xi)$ [227],

$$
\Pi(\xi)=\sum_{k}\left(\phi_{k}(\xi) a_{k}+\phi_{k}^{*}(\xi) a_{k}^{\dagger}\right)
$$

where $\xi$ denotes arbitrary coordinates. The operators $a_{k}$ and $a_{k}^{\dagger}$ associated with the modes are annihilation and creation operators, respectively, which obey the standard canonical commutation relations

$$
\left[a_{k}, a_{k^{\prime}}^{\dagger}\right]=\delta_{k, k^{\prime}}
$$

The dispersion relation for these phononic modes is given by $\omega_{k}=c_{s}|\mathbf{k}|$, with $c_{s}$ is the speed of sound.

\footnotetext{
${ }^{3}$ The healing length, also known as coherence length, for a one-dimensional BEC describes the distance over which the wave function tends to its bulk value when subjected to a localised perturbation.
} 
In the approximation we are considering for the BEC, see appendix E, the phononic modes obey a massless Klein-Gordon equation

$$
\square \Pi(\xi)=0 .
$$

The d' Alembertian operator (possibly in curved space-time) is defined as

$$
\square=\frac{1}{\sqrt{-\mathfrak{g}}} \frac{\partial}{\partial x_{a}}\left(\sqrt{-\mathfrak{g}} \mathfrak{g}^{a b} \frac{\partial}{\partial x_{b}}\right)
$$

and it depends on an effective space-time metric $\mathfrak{g}_{a b}-$ with $\mathfrak{g}=\operatorname{det} \mathfrak{g}_{a b}$. The effective, emergent metric $\mathfrak{g}_{a b}$ is defined as [107, 284]

$$
\mathfrak{g}_{a b}=\left(\frac{n_{0}^{2} c_{s}^{-1}}{\rho_{0}+p_{0}}\right)\left[g_{a b}+\left(1-\frac{c_{s}^{2}}{c^{2}}\right) V_{a} V_{b}\right]
$$

Note that the acoustic metric $\mathfrak{g}_{a b}$ is a function of background mean field properties of the BEC, such as the number density $n_{0}$, the energy density $\rho_{0}$ and the pressure $p_{0}$. The effective curvature naturally arises from decoupling the field equations of the background mean field and the quantum fluctuations. $V_{a}$ is the BEC 4 - velocity with respect to the laboratory reference frame, while $g_{a b}$ is the background, physical four-dimensional space-time metric that in general may be curved.

Strictly speaking, in the experiment we propose, the BECs move in a Schwarzschild metric. However, due to the smallness of the Schwarzschild radius of the earth, $r_{s} \simeq 9 \times 10^{-3} \mathrm{~m}$, it is reasonable to assume that the space-time is flat, i.e. that one can ignore tidal effects. The BECs are inertial while they free fall in a circular orbit, and in this case we use Minkowski coordinates $(t, \vec{x})$. In order to change the orbit of one of them, so that it undergoes a change in gravitational potential, acceleration is required. We consider the satellite to undergo a single change in velocity, that is a single period of uniformly accelerated motion. The direction, intensity and duration uniquely determines the new orbit.

Therefore, we consider a Rindler transformation of the Bogoliubov modes since Rindler coordinates are suitable to describe periods of uniformly accelerated motion (see Appendix B). We choose the comoving frame $V_{a}=(1 ; 0,0,0)$ since we want to describe the effects in the rest frame of the BEC. Under these conditions we obtain an effective metric $\mathfrak{g}_{a b}$ which is conformally flat.

We would like to remark here that, in our derivation of the acoustic metric we have considered a $3+1$-dimensional, background physical metric $g_{a b}$ from the very beginning - that is we are considering a relativistic BEC [107]. This is not, in general, the case in the analogue models programme, where one starts with some 
system defined in the usual Euclidean three-dimensional metric, and obtains, after suitable approximations, a set of equations defined in four dimensions [56]. By contrast, we have chosen to have a background four-dimensional metric so that we could directly implement a Rindler transformation for the coordinates - when one of the two BECs undergoes a constant accelerated motion - and, through the acoustic metric, this accelerated motion can affect the phononic modes, that, to all intents and purposes, experience only the acoustic metric.

Given the approximations we are considering, the effective metric, as experienced by a phononic, in Minkowski coordinates, is

$$
\mathfrak{g}_{a b}=\left(\frac{n_{0}^{2} c_{s}^{-1}}{\rho_{0}+p_{0}}\right)\left[\begin{array}{cccc}
-\frac{c_{s}^{2}}{c^{2}} & & & \\
& 1 & & \\
& & 1 & \\
& & & 1
\end{array}\right]
$$

\subsubsection{Inertial and accelerated motion}

Having a description of the BEC on a space-time metric enables us to describe it while it undergoes inertial and uniformly accelerated motion. In the inertial case, we consider Minkowski coordinates $(t, x)$ where the line element is given by

$$
d s^{2}=g_{\mu \nu} d x^{\mu} d x^{\nu}=-c^{2} d t^{2}+d x^{2} .
$$

As we have seen in equation E.8, if $g_{a b}$ is flat, the effective metric $\mathfrak{g}_{a b}$ is also flat when the spatial flow velocities vanish. In this case the phonons obey a flat-space KleinGordon equation which takes the form of a wave equation in Minkowski coordinates with propagation velocity $c_{s}$. The solutions to the equation, denoted $\phi_{n}(t, x)$ with $n \in \mathbb{N}$, form an orthonormal set of modes in terms of which the field $\Pi(t, x)$ can be expanded,

$$
\Pi(x)=\sum_{n}\left[\phi_{n}(t, x) a_{n}+\text { h.c. }\right]
$$

Here $a_{n}, a_{n}^{\dagger}$ are the annihilation and creation operators associated to the modes $\phi_{n}$.

For the sake of simplicity, we consider a quasi one dimensional BEC. Suitable close to hard-wall boundary conditions [127, 137, 212] allow us to consider a spectrum similar to the well-known spectrum of an optical cavity given by 


$$
\omega_{n}=2 \pi \times \frac{n c_{s}}{L}
$$

where $L$ is the length of the cylinder.

For periods of uniform acceleration, Rindler coordinates $(\eta, \chi)$ are a convenient choice of coordinates [70]. They are related to the Minkowski coordinates by the following transformation

$$
t=\frac{\chi}{c} \sinh \eta, \quad x=\chi \cosh \eta
$$

where $\chi>0$ has dimension length and $\eta \in \mathbb{R}$ is the dimensionless Rindler time. The line element in these coordinates is $d s^{2}=-\chi^{2} d \eta^{2}+d \chi^{2}$. A uniformly accelerated observer follows a trajectory of constant $\chi=\chi_{o}$ and its proper time is given by $\tau=\frac{c}{a} \eta$, where $a=\frac{c^{2}}{\chi_{o}}$ is its proper acceleration.

If the acceleration is such that to keep the density of the BEC homogeneous, as it is in our casf ${ }^{4}$, we can still define acoustic metric in terms of Rindler coordinates. Therefore, when the BEC undergoes uniform acceleration, the phononic BEC field obeys again a Klein-Gordon equation which takes the form in this case of a wave equation in Rindler coordinates. The Rindler solutions are denoted by $\tilde{\phi}_{n}(\eta, \chi)$ with $n \in \mathbb{N}$ and the field expansion is given by

$$
\Pi(\eta, \chi)=\sum_{n}\left[\tilde{\phi}_{n}(\eta, \chi) \tilde{a}_{n}+\text { h.c. }\right] .
$$

The operators $\tilde{a}_{n}, \tilde{a}_{n}^{\dagger}$ are now the annihilation and creation operators associated to the Rindler modes $\tilde{\phi}_{n}$.

\subsection{Bogoliubov transformations, the covariance matrix formalism and entanglement}

In our work we consider a condensate that is initially inertial, then undergoes a change in the gravitational field potential as it changes into a different orbit, and is finally inertial again. The change in field potential corresponds to a period of uniform acceleration. The creation and annihilation operators in the initial and final regions are respectively denoted by $a, a^{\dagger}$ and $\hat{a}, \hat{a}^{\dagger}$, and are related through a Bogoliubov transformation [70],

$$
\left(\begin{array}{c}
\hat{a} \\
\hat{a}^{\dagger}
\end{array}\right)=\left(\begin{array}{cc}
\alpha & \beta \\
\beta^{*} & \alpha^{*}
\end{array}\right) \cdot\left(\begin{array}{c}
a \\
a^{\dagger}
\end{array}\right),
$$

\footnotetext{
${ }^{4}$ See appendix $\mathrm{E}$ section $\mathrm{E}$ for detailed discussion.
} 
where $\alpha_{n m}=\left(\phi_{n}, \hat{\phi}_{m}\right)$ and $\beta_{n m}=-\left(\phi_{n}, \hat{\phi}_{m}^{*}\right)$ are Bogoliubov coefficients. Here $(\cdot, \cdot)$ denotes the inner product. $\phi$ and $\hat{\phi}$ are Minkowski mode solutions in the initial and final regions, respectively. These Bogoliubov coefficients are functions of the Bogoliubov coefficients between the Rindler and Minkowski modes given by ${ }_{0} \alpha_{n m}=\left(\phi_{n}, \tilde{\phi}_{m}\right)$ and ${ }_{0} \beta_{n m}=-\left(\phi_{n}, \tilde{\phi}_{m}^{*}\right)$ and of phases acquired during the period of uniform acceleration where the condensate undergoes free evolution (for more details see [74]). The coefficients ${ }_{0} \alpha_{m n}$ account for mode mixing within the moving condensate, while ${ }_{0} \beta_{m n}$ account for particle pair production.

The Bogoliubov coefficients can be computed analytically in terms of the parameter

$$
h=a L / c_{s}^{2},
$$

where $a$ is the acceleration of the BEC, $L$ is the dimension of the trap, and $c_{s}$ is the speed of sound. For the system we are considering, we have that $a \sim 10^{-3} \mathrm{~m} / \mathrm{s}^{2}$, $L \sim 10^{-3} \mathrm{~m}$ and $c_{s} \sim 10^{-2} \mathrm{~m} / \mathrm{s}$ (however the speed of sound depends on the specific $\mathrm{BEC}$ one wants to consider). If $h \ll 1$, as it is in our case, the Bogoliubov coefficients 9.12) can be expanded in series as

$$
\alpha_{m n}=\alpha_{m n}^{(0)}+\alpha_{m n}^{(1)}+\alpha_{m n}^{(2)}+\mathcal{O}\left(h^{3}\right)
$$

and

$$
\beta_{m n}=\beta_{m n}^{(1)}+\beta_{m n}^{(2)}+\mathcal{O}\left(h^{3}\right),
$$

where the superscript ${ }^{(n)}$ denotes quantities that are proportional to $h^{n}$ [74, 124]. In the case we consider here, the Bogoliubov coefficients to first order in $h$ are given by 74,124

Zeroth order: $\quad \alpha_{m n}^{(0)}=\delta_{m n} e^{-i \Omega_{n} \Delta \tau}$

$$
\beta_{m n}^{(0)}=0 \text {. }
$$

First order: $\quad \alpha_{m n}^{(1)}=e^{-i\left(\Omega_{n}-\Omega_{m}\right) \Delta \tau}{ }_{0} \alpha_{m n}^{(1)}$

$$
\begin{aligned}
& =e^{-i\left(\Omega_{n}-\Omega_{m}\right) \Delta \tau} \frac{\left(-1+(-1)^{(m-n)}\right) \sqrt{m n}}{\pi^{2}(m-n)^{3}} ; \\
\beta_{m n}^{(1)} & =e^{i\left(\Omega_{n}-\Omega_{m}\right) \Delta \tau}{ }_{0} \beta_{m n}^{(1)} \\
& =e^{i\left(\Omega_{n}-\Omega_{m}\right) \Delta \tau} \frac{\left(1-(-1)^{m-n}\right) \sqrt{m n}}{\pi^{2}(m+n)^{3}} .
\end{aligned}
$$

Here $\Omega_{n}$ are the frequencies of the modes as measured by a comoving accelerated observer, and $\Delta \tau$ is the proper time spent while accelerating. 


\subsubsection{Covariance matrix}

Let us now consider the covariance matrix formalism, in which all the relevant information about the state is encoded in the first and second moments of the field. In particular, the second moments are described by the covariance matrix $\sigma_{i j}=\left\langle X_{i} X_{j}+X_{j} X_{i}\right\rangle-2\left\langle X_{i}\right\rangle\left\langle X_{j}\right\rangle$, where $\langle$.$\rangle denotes the expectation value and the$ quadrature operators $X_{i}$ are the generalized position and momentum operators of the field modes given by $X_{2 n-1}=\frac{1}{\sqrt{2}}\left(a_{n}+a_{n}^{\dagger}\right)$ and $X_{2 n}=\frac{-i}{\sqrt{2}}\left(a_{n}-a_{n}^{\dagger}\right)$. In appendix $F$ the reader can find a more thorough description of the covariance matrix. Every unitary transformation in Hilbert space that is generated by a quadratic Hamiltonian can be represented as a symplectic matrix $S$ in phase space. These transformations form the real symplectic group $S p(2 n, \mathbb{R})$, the group of real $(2 n \times 2 n)$ matrices that leave the symplectic form $\Omega$ invariant, i.e., $S \Omega S^{T}=\Omega$, where $\Omega=\bigoplus_{i=1}^{n} \Omega_{i}$ and

$$
\Omega_{i}=\left(\begin{array}{cc}
0 & 1 \\
-1 & 0
\end{array}\right) .
$$

The time evolution of the field, as well as the Bogoliubov transformations, can be encoded in this symplectic structure (for details see [123]). The covariance matrix, after a symplectic transformation, is given by $\tilde{\sigma}=S \sigma S^{T}$. In our proposal two space experimentalists, let us call them Valentina and Yuri, are initially inertial and prepare an entangled two-mode squeezed stat $5^{5}$ of their phononic modes $k$ and $k^{\prime}$, each one of them in their respective condensate. We assume that all other modes in both condensates are in the vacuum state. Since the trace operation over a set of modes is implemented in this formalism by deleting the rows and columns associated to those modes, we find that the covariance matrix of the reduced state for the modes $k$ and $k^{\prime}$ is given by

$$
\sigma_{k k^{\prime}}=\left(\begin{array}{cc}
\cosh (2 r) \mathbb{1}_{2} & \phi_{k k^{\prime}} \\
\phi_{k k^{\prime}} & \cosh (2 r) \mathbb{1}_{2}
\end{array}\right) .
$$

Here

$$
\phi_{k k^{\prime}}=\left(\begin{array}{cc}
\sinh (2 r) & 0 \\
0 & -\sinh (2 r)
\end{array}\right),
$$

and $r>0$ is the squeezing parameter of the state. The matrix $\mathbb{1}_{2}$ is the $2 \times 2$ identity matrix. During inertial and uniformly accelerated segments of motion, the field modes only undergo free evolution. Therefore, the transformation in this case is simply composed of local rotations with angles $\omega_{k} t$ and $\omega_{k^{\prime}} t$, where $\omega_{k}$ and $\omega_{k^{\prime}}$ are the

\footnotetext{
${ }^{5} \mathrm{~A}$ brief definition of squeezed state can be found in appendix $\mathrm{F}$.
} 
angular frequencies of the modes $k$ and $k^{\prime}$ respectively. The covariance matrix after Valentina remains inertial and Yuri undergoes a single period of uniform acceleration to move to a different orbit is given by ${ }^{6}$

$$
\tilde{\sigma}_{k, k^{\prime}}=\left(\begin{array}{cc}
C_{k k} & C_{k k^{\prime}} \\
C_{k k^{\prime}}^{T} & C_{k^{\prime} k^{\prime}}
\end{array}\right)
$$

where $C_{k k}=\cosh (2 r) \mathbb{1}_{2}, C_{k k^{\prime}}=\phi_{k k^{\prime}} \mathcal{M}_{k^{\prime} k^{\prime}}^{T}$ and

$$
C_{k^{\prime} k^{\prime}}=\cosh (2 r) \mathcal{M}_{k^{\prime} k^{\prime}} \mathcal{M}_{k^{\prime} k^{\prime}}^{T}+\sum_{n \neq k^{\prime}} \mathcal{M}_{k^{\prime} n} \mathcal{M}_{k^{\prime} n}^{T}
$$

The $2 \times 2$ matrices $\mathcal{M}$ encode the Bogoliubov coeffcients given by Eq. (9.16),

$$
\mathcal{M}_{n m}=\left(\begin{array}{cc}
\operatorname{Re}\left(\alpha_{m n}^{(0)}+\alpha_{m n}^{(1)}-\beta_{m n}^{(1)}\right) & \operatorname{Im}\left(\alpha_{m n}^{(0)}+\alpha_{m n}^{(1)}+\beta_{m n}^{(1)}\right) \\
-\operatorname{Im}\left(\alpha_{m n}^{(0)}+\alpha_{m n}^{(1)}-\beta_{m n}^{(1)}\right) & \operatorname{Re}\left(\alpha_{m n}^{(0)}+\alpha_{m n}^{(1)}+\beta_{m n}^{(1)}\right)
\end{array}\right)
$$

Here Re and Im denote the real and imaginary parts, respectively.

\subsubsection{Measurement of the entanglement - Negativity}

A number of computable measures of entanglement exist for Gaussian states in terms of the smallest symplectic eigenvalue $\nu_{-}$of the partial transposition of $\tilde{\sigma}$. Here we are interested in computing the negativity 7 of the state $\tilde{\sigma}_{k k^{\prime}}$ to understand how entanglement is affected when Yuri has changed his condensate into an orbit with different gravitational potential. In this case the negativity is given by

$$
N=\max \left[0, \frac{1-\nu_{-}}{2 \nu_{-}}\right]
$$

where

$$
\nu_{ \pm}=\sqrt{\frac{\Delta\left(\tilde{\sigma}_{k k^{\prime}}\right) \pm \sqrt{\Delta^{2}\left(\tilde{\sigma}_{k k^{\prime}}\right)-4 \operatorname{det} \tilde{\sigma}_{k k^{\prime}}}}{2}}
$$

and $\Delta\left(\tilde{\sigma}_{k k^{\prime}}\right)=\operatorname{det} C_{k k}+\operatorname{det} C_{k^{\prime} k^{\prime}}-2 \operatorname{det} C_{k k^{\prime}}$.

The negativity for this particular state we are considering is given by [7]

$$
N^{(0)}=\max \left[0, \frac{1}{2}\left(e^{2 r}-1\right)\right]
$$

\footnotetext{
${ }^{6}$ In general, the initial covariance matrix $\sigma$, that includes all the modes, is transformed after the change in orbit into $\tilde{\sigma}=S \sigma S^{T}$, where $S$ is a symplectic matrix that encodes the time evolution of the system.

${ }^{7}$ An extensive definition of the negativity measure can be found in appendix $\mathrm{F}$
} 
where the condensate undergoes free evolution. After the change of orbit, and using equations 9.15 to 9.25 , we find that the entanglement is now given by $N=N^{(0)}+N^{(2)} h^{2}+\mathcal{O}\left(h^{4}\right)$. More specifically:

$$
N=\max \left[0, N^{(0)}\left(1-e^{2 r}\left(f_{k^{\prime}}^{\alpha}+f_{k^{\prime}}^{\beta}\right) h^{2}\right)-e^{2 r} f_{k^{\prime}}^{\beta} h^{2}\right]
$$

and

$$
f_{k^{\prime}}^{\alpha}=\sum_{n}\left|\alpha_{k^{\prime} n}^{(1)}\right|^{2} \quad, \quad f_{k^{\prime}}^{\beta}=\sum_{n}\left|\beta_{k^{\prime} n}^{(1)}\right|^{2}
$$

Here $f_{k^{\prime}}^{\alpha}, f_{k^{\prime}}^{\beta}$ are functions of the Bogoliubov coefficients that depend periodically on the difference of the gravitational field strength 8 , see fig. 9.2. Note that $N^{(0)}$ is the entanglement of the initial state given by equation $(9.26) . N$ is always smaller than $N^{(0)}$, since the entanglement is degraded by mode mixing and particle creation [125, 302. This degradation effect becomes observable for large enough, but still perturbative, values of $h, h^{2} \simeq 0.05$ [125].

In optical cavities, these values of $h$ are obtained with accelerations of $10^{23} \mathrm{~m} / \mathrm{s}^{2}$ - see equation $(9.13)$ - while in superconducting cavities, the corresponding order of magnitude is $10^{17} \mathrm{~m} / \mathrm{s}^{2}$, which can be achieved by non-mechanical means 125 , 302. In the case under study here namely, BECs, the typical values $L \simeq 100 \mu \mathrm{m}$ and $c_{s}=1 \mathrm{~mm} / \mathrm{s}$ give rise to $a \simeq 10^{-3} \mathrm{~m} / \mathrm{s}^{2}$. In figure 9.2 we can see the behaviour of the negativity when the system undergoes a change of gravitational potential. The different lines represents different accelerations, all within the parameter values that allow us to define an effective, emergent metric.

\subsection{Experimental setup}

We now assess the feasibility of testing the degradation of entanglement due to orbit changes with a space-based experiment using a pair of nanosatellites. Nanosatellites are fully functional spacecraft with a mass of 1 to $10 \mathrm{~kg}$. The use of conventional off the shelf (COTS) parts, component miniaturization, and standardized systems means that they are a comparatively low cost avenue to space. Capabilities such as power, attitude and position control, propulsion, optics, communication, and autonomous operation are under active development which greatly expands the missions which may be undertaken within the mass and volume envelope of the

${ }^{8}$ A full calculation for the coefficients $f_{k^{\prime}}^{\alpha}$ and $f_{k^{\prime}}^{\beta}$ can be found in [74], sec. II, equations (3) and (4). The periodicity of the negativity with respect to the acceleration is a general feature that holds beyond perturbation theory for the scalar massless field in $(1+1)$-dimension, as explained in 74 . 


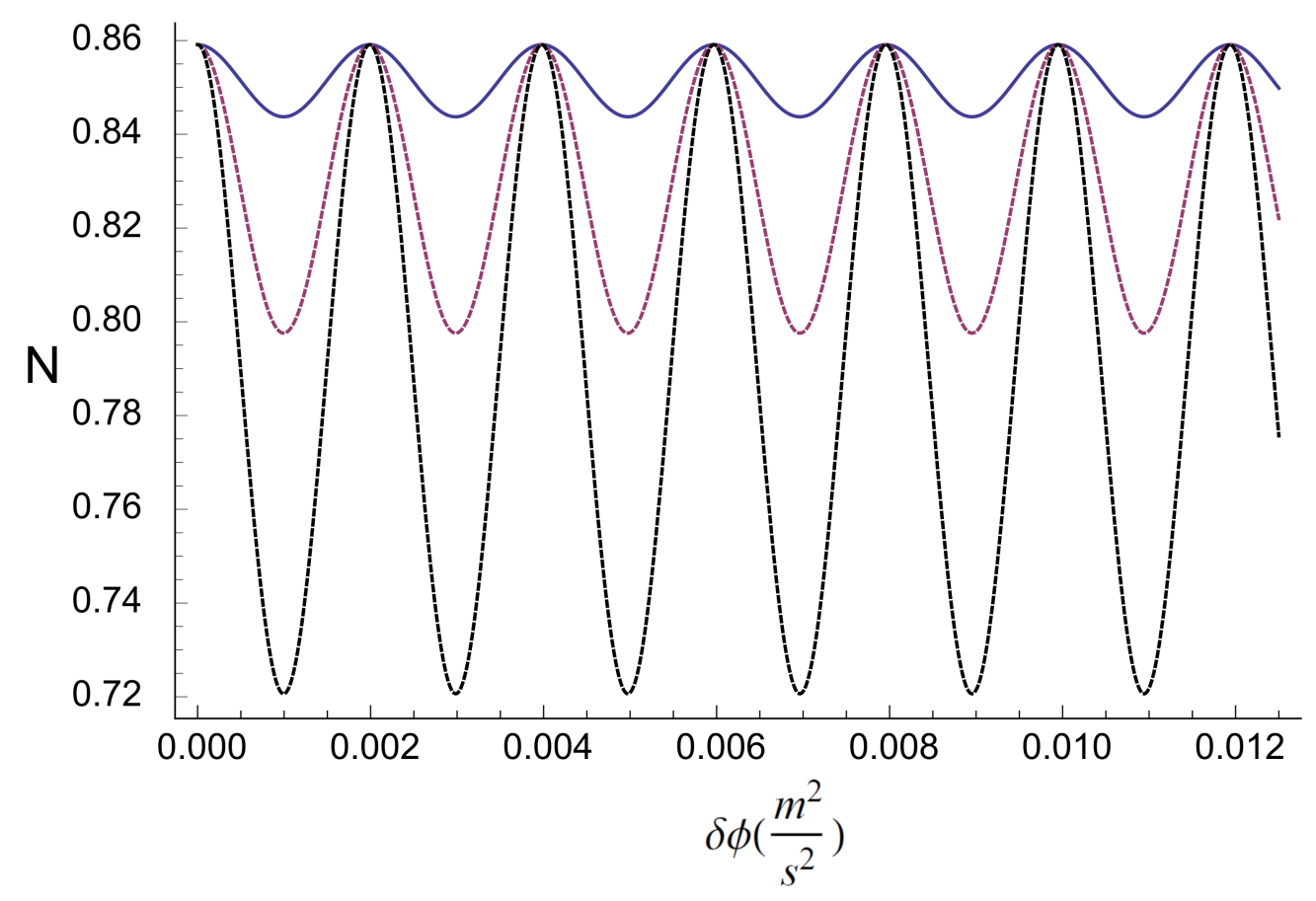

Figure 9.2: Negativity $N$ vs. difference in gravitational field strength between initial and final orbits $\delta \phi$, after the first change in velocity $\Delta v_{l}$. The acceleration of the satellite is $a=10^{-3} \mathrm{~m} / \mathrm{s}^{2}$ (solid, blue), $a=2 \cdot 10^{-3} \mathrm{~m} / \mathrm{s}^{2}$ (red,dashed), $a=3 \cdot 10^{-3} \mathrm{~m} / \mathrm{s}^{2}$ (black, dotted) while $L=100 \mu \mathrm{m}$, $c=1 \mathrm{~mm} / \mathrm{s}$, giving rise to $h^{2} \simeq 0.05$ and $\Omega_{1}=2 \pi \times 50 \mathrm{~Hz}$. The initial squeezing is $r=1 / 2$ and the duration of the acceleration is of the order of $0.1 \mathrm{~s}$. 
nanosatellite platform. At the same time, quantum experiments have also become more compact which makes it feasible to place them on small satellites [201].

\section{The nanosatellites}

An example of the capability required for such an experiment is the pair of CanX-4 and CanX-5 [41, 248] satellites due to launch in 2014. These are built according to the Generic Nanosatellite Bus (GNB) specification which consists of a $20 \mathrm{~cm}$ a side cube with a mass of approximately $7.5 \mathrm{~kg}$. Typically, such a spacecraft has a mission payload volume of 1.8 litres and mass of $2 \mathrm{~kg}$. The CanX-4/5 pair will demonstrate formation flying in orbit and are each equipped with high precision differential GPS receivers for centimetre accuracy relative positioning determination, and a single axis thruster allowing orbit changes. The latter consists of the Canadian Nanosatellite Advanced Propulsion System (CNAPS) and has a rated thrust of $20 \mathrm{mN}$ and an $I_{s p}$ of $35 \mathrm{~s}$ resulting in a $\Delta V$ of $11.1 \mathrm{~m} / \mathrm{s}$. Therefore the satellites can accelerate with the constant acceleration $a \simeq 10^{-3} \mathrm{~m} / \mathrm{s}^{2}$ necessary to make the predicted effects observable. Figure 9.3 illustrates the satellites' layout 9 .

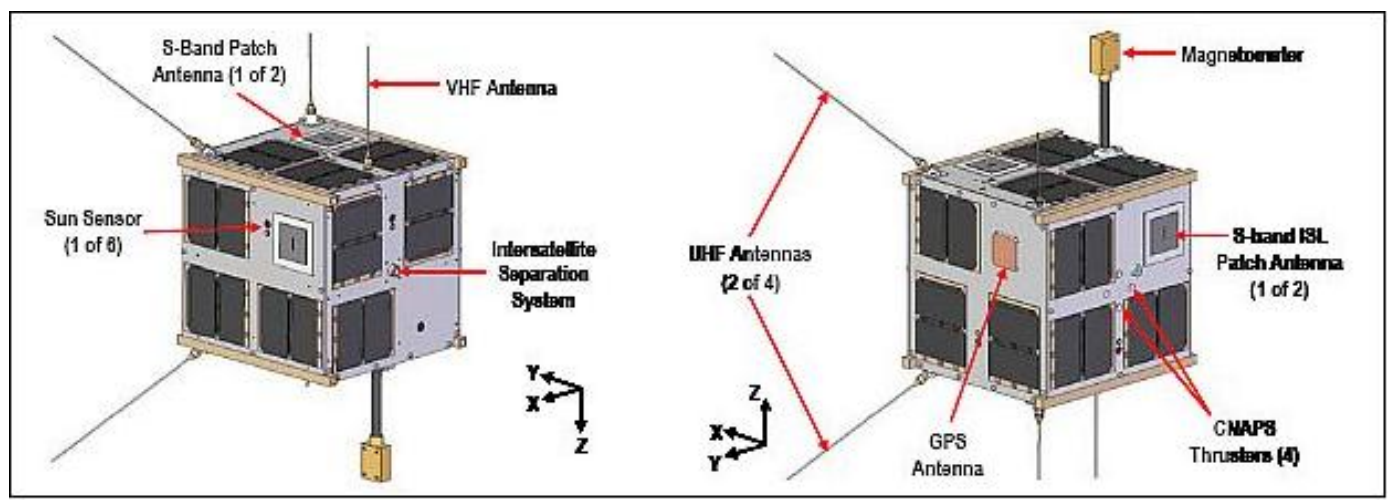

Figure 9.3: Illustration of the CanX-4 and -5 nanosatellites - shown in opposing views. Image courtesy of UTIAS/SFL.

\footnotetext{
${ }^{9}$ For the interested reader, we recommend the following webpages: https://directory.eoportal.org/web/eoportal/satellite-missions/c-missions/canx-4-5 http://www.utias-sfl.net
} 


\section{Preparing the BEC}

Let us consider a pair of satellites, such as CanX4 and CanX5, moving along the same circular orbit. Each satellite contains a BEC with initially entangled phonon modes. Such an entangled state can be prepared in several ways. For instance, the BECs can be made to interact through Bragg scattering with two separated laser beams that excite quasi-particles of specific momenta in each condensate. Entanglement is then produced by performing projective measurements on the scattered light beams [96]. Atom-light entangling techniques can also be used, where via electromagnetically induced transparency and subsequent projective measurements, the entanglement is transferred from two probe laser beams to two spatially separated BECs [188. Similar techniques can also be applied by considering two separate BECs in two distinct, high-finesse optical cavities, on which two quantum correlated light fields are incident, hence transferring their quantum correlated state to the two BECs, [191. If the BEC is in an initial thermal state instead of the vacuum state, the amount of initial squeezing and entanglement that can be generated will be lower 73. In order to generate a squeezing $r=1 / 2$ and frequencies of $100 \mathrm{~Hz}$, the BEC should be cooled down to a few nK. Finally, notice that the experimental setup required to create and hold the $\mathrm{BEC}$ can be as small as $0.5 \mathrm{~L}$ [100]. Important efforts are currently taking place to load and maintain a BEC on a chip device in space [245].

\section{Change of orbit}

The effects predicted in this work arise when a satellite undergoes a change of circular orbit, determined by the difference in gravitational field strength between the initial and final orbits. As an example, the change of orbit can be achieved in an efficient and elegant manner by means of a Hohmann transfer orbit [144, 145] (see Fig.1). The procedure is the following. First a change of velocity $\Delta v_{l}$ moves the satellite to an elliptic orbit. Then the satellite navigates half of this new orbit, before finally a second velocity kick $\Delta v_{h}$ puts the satellite back into a circular orbit. The difference between the radius of the initial orbit $r_{l}$ and the radius of the final orbit $r_{h}$ determines the magnitude of the velocity kicks through the relations

$$
\Delta v_{l}=\sqrt{\frac{G M}{r_{l}}}\left(\sqrt{\frac{2 r_{h}}{r_{l}+r_{h}}}-1\right), \quad \Delta v_{h}=\sqrt{\frac{G M}{r_{h}}}\left(1-\sqrt{\frac{2 r_{l}}{r_{l}+r_{h}}}\right) ;
$$

where $G$ is Newton's gravitational constant and $M$ is the mass of the earth. In particular, assuming a small change of altitude $r_{h}=r_{l}+\delta r$ with $\delta r \ll r_{l}$, we find 


$$
\Delta v_{l} \simeq \Delta v_{h} \simeq \sqrt{\frac{G M}{r_{h}}} \frac{\delta r}{4 r_{h}} \simeq \sqrt{\frac{r_{h}}{G M}} \frac{\delta \phi}{4} \simeq 3 \times 10^{-3} \mathrm{~m} / \mathrm{s}
$$

for a Low Earth Orbit (LEO) of $r_{h}=R_{e}+400 \mathrm{~km}-R_{e}$ being the radius of the earth and $\delta \phi$ the difference in gravitational field strength between the initial and final orbits. Therefore, for constant acceleration, each radial distance between circular orbits is related to a different duration of the acceleration. The whole manoeuvre takes a half-period $P / 2$ of the elliptical transfer orbit $P \simeq 2 \pi \sqrt{r_{h}^{3} / G M} \simeq 5000 s$ which is larger than the average lifetime of a BEC. However, the degradation of the entanglement takes place immediately after the first change in velocity, and can be observed during the navigation of the transfer orbit. Equations 9.27) and (9.29) imply that the entanglement oscillates with the radial distance between the initial and final orbit, or equivalently, with the difference in the gravitational strength. In figure 9.2 we show that, for realistic experimental parameters, oscillations have a significant amplitude and a period of around $2 \mathrm{~m}$, meaning that almost any change of orbit would lead to an observable effect on the initial quantum entanglement. Note that the duration of the acceleration in the plot is of the order of $0.1 \mathrm{~s}$. The maximum change of velocity is $\Delta v_{l} \simeq 10^{-3} \mathrm{~m} / \mathrm{s}$ well within reach of current technologies since CanX4 and CanX5 are capable of achieving maximum changes of velocities of $\Delta v=11.1 \mathrm{~m} / \mathrm{s}$. Much larger changes of orbit can be considered for which the behaviour of entanglement as a function of difference in gravitational strength is shown in Fig. (9.2). Since CanX4 and CanX5 are designed to determine positions with an accuracy in the centimetre range, they seem ideal devices to analyse the dependence of entanglement with the radial distance.

\section{Experiment readout}

The readout of the quantum correlations might be performed in a manner similar to the experiment in [167], where upon releasing the condensate trapping potential, each quasi-particle deposits its momentum on an atom and velocities are measured by a position sensitive single-atom detector. Unfortunately this technique is destructive and many shots of the experiment would be necessary to achieve the required statistics. An alternative method consists in using atomic quantum dots or optical lattices coupled to each condensate to probe the reduced field states of each condensate [246]. This method enables one to perform several thousands of correlated measurements within the coherence time of the entangled state we considered. 


\subsection{Conclusions regarding the possible effects of motion and gravity on quantum physics}

In conclusion, we have shown that changes in the gravitational field potential produce effects on quantum entanglement that are in principle observable in space-based experiments. In particular, we have shown that entanglement between two BECs inside separate satellites can be degraded when one of them undergoes a change of orbit. Entanglement oscillates periodically with the difference in gravitational potential of the orbits. Therefore, by accurately controlling the satellite positions, it is possible to find situation in which entanglement is conserved. Our results shed light on fundamental aspects in the overlap between quantum theory and relativity by working within QFT, a framework which incorporates appropriately these theories in regimes where satellites operate. These results will inform future spacebased quantum technologies, including quantum key distribution and other quantum cryptographic experiments. A comprehensive understanding of relativistic effects on quantum properties will enable us not only to make the necessary corrections to the technologies they affect, but also opens up the possibility of using relativistic effects as resources.

In honour of Valentina Tereshkova and Yuri Gagarin, who were the first woman and man to go to space. 


\section{Chapter 10}

\section{Summary and discussion}

In this thesis work we have presented some results that are related to the quest of a quantum theory of gravity. As we have seen, besides the orthodox ways of addressing this problem, other viable ways include testing those phenomena that lie at the interface between quantum physics and general relativity (or curved spacetime). We have explained that the motivation behind this alternative approach is trying to find the next step to take toward a quantum theory of/for gravitation ${ }^{1}$.

In the first part of thesis we have briefly addressed the possibility that, at energies much higher that those reachable by current technology, physics may break Lorentz invariance. We have then addressed this matter in the context of local physics, by considering transformations between inertial frames. We have seen that the von Ignatoswki argument established a close relation between the relativity principle and the group structure for the set of transformations between inertial frame (Lorentz group). By reversing this logic, we have seen that, in order to break Lorentz symmetry, the key step in is to renounce the relativity principle, de facto introducing a preferred frame that we have called the aether frame. Even when renouncing the relativity principle, we have found that substantial physics can still be predicted. For instance we have seen that the set of transformations between generic inertial frames form a group/pseudogroup.

We have therefore established the transformation rules for energy and momentum to be, in general, affine - that is linear plus an offset term. By comparison with the standard case of Galileo group and Lorentz group, we have seen that the offset plays a different role when considering different regimes.

\footnotetext{
${ }^{1}$ As the reader may have noticed, throughout this thesis work we have used the expression quantum theory of/for gravitation. With this we mean to say that a theory that describes the microscopic states of space and time may not arise from general relativity, while these microscopic degrees of freedom may be defined in a completely independent way.
} 
With these tools, we have developed two minimalist Lorentz violating models. In the first one, we have assumed the physics of clock and rulers to be Lorentz symmetric, implying that space and time transform with the usual Lorentz transformations, while the energy-momentum relation, for at least some of the particles, is not Lorentz invariant. The second model presents the simplest violation of Lorentz invariance at the level of the transformations between inertial frames. In this case, we have imposed linearity, isotropy and reciprocity for the transformations between inertial frames but not the relativity principle.

Following along these lines, we have also developed some decay and scattering threshold theorems in a Lorentz symmetry breaking scenario. In particular, the only assumption we have made is the existence of some relation $E(p)$ between the energy and the momentum, without assuming its isotropy and monotonicity. Our analysis has been carried out by solely considering the kinematically allowed region, and consequent threshold structure coming from the boundaries of the kinematically allowed region, and has turned out to be much more complicated than in the Lorentz invariant case. In particular we could still make some limited number of truly general statements. We have tried to carefully delineate exactly which assumptions are central to which results, concentrating on those results that depend only on the existence of a homogeneous space-time, and adding extra assumptions only when essential to obtaining specific specialised results.

In the second part we have presented an alternative way, in some sense, to approach the investigation of a quantum theory of gravity: The space-time thermodynamics. In this case space-time is intended as being an emergent mean-field approximation of underlying microscopic degrees of freedom, a hypothesis developed in 1964 by Andrei Sakharov. This conjecture was reinforced after the formulation of Hawking radiation, and the realisation that the four laws of black hole dynamics were actually identical to those of thermodynamics. Following this path, in 1995 Ted Jacobson showed that the Einstein equation can be obtained as an equation of state when considering the proportionality between the entropy and the area of a local causal horizon (a Rindler horizon in his derivation) and the Clausius relation for the entropy. In this part of the thesis, we have considered a similar thermodynamic system to that considered by Jacobson in his derivation, and instead of proceeding with the derivation of the Einstein equation, we have generalised the system itself to a generic bifurcate null surface. In this way, we have not only paved the way for exploring the potential derivation of the Einstein equation in the case of a more generic causal horizon; in addition we have established the virtual nature of Clausius 
entropy for bifurcate null surfaces.

Given the importance that entropy seems to have, together with very little clarity concerning the equivalence of its several definitions existing in the literature, we have explored some of its properties in the simplified case of single-channel Shannon entropy - in the information theoretic sense. The results we have found are that to obtain infinite Shannon entropy an infinite number of states must have non-zero but arbitrarily small probability, that the Shannon entropy can never be too divergent, and that in a suitable technical sense infinite Shannon entropy is never worse than logarithmic in the cutoff. For this reason, large Shannon entropies cannot be tightly localised in state space and must invariably come from exponentially large regions of the state space.

In the last part of the thesis we have described a proposal for an experiment that would test the effect of acceleration and curved space-time on quantum physics. As we have explained in more detail, this kind of approach may be considered as an alternative way to find some phenomenological evidence of quantum gravity in which we can investigate the behaviour of typical quantum physics phenomena such as entanglement - at large scales. A second purpose lies in the opportunity to implement quantum technology for space-based communication.

In particular, the system we have considered is composed of two BECs, set into two nano-satellites, that at first follow the same geodesic. Afterwards, one of the two undergoes an accelerated motion before it settles back into a geodesic orbit. We have explained that the reason behind the choice of BECs lies in the existence of an effective, acoustic metric for the phonons - in the range of acceleration we are considering - and the fact that the very slow interaction propagation seems to enhance the effect we want to see. The final result we have found is that the quantum entanglement between two phononic modes in two separated BECs is degraded when one of them undergoes a change of orbit, and oscillates periodically with the difference in gravitational potential of the orbits. 
Part IV

Appendices 



\section{Appendix A}

\section{Lorentz symmetry breaking}

\section{A.1 Double special relativity and its applicability to our model}

Regarding the possibility of working with DSR (doubly special relativity, distorted special relativity), it will soon become clear that DSR falls outside our framework. (For general background see [18, 19, 21, 172, 200].) For DSR-like models a key issue is that after a decade of work on this topic, and despite significant ongoing efforts, there is still no clear universally accepted consensus as to how space and time transform between inertial frames $[8,11,13$, 17, 97, 129, 130, 135, 143, 147152, 234, 241, 254, 260] - there is not even any clear consensus on whether or not photon velocities are momentum-dependent in general DSR frameworks [94, 128]. There are also suggestions to the effect that the "D" in DSR should be attributed to adopting a modified theory of measurment [200].

However, there is reasonable consensus that the energy-momentum transformations of DSR-like theories are generically of the form [172]

$$
P \rightarrow \bar{P}=f\left(L f^{-1}(P)\right)
$$

for $L$ an ordinary linear Lorentz transformation and $f(P)$ some nonlinear function on energy-momentum space. Since these energy-momentum transformations are not affine, the considerations we have found in 4.3 imply that DSR-like theories (insofar as they are internally consistent), must at the very least exhibit other oddities - such as a breakdown in locality, or a breakdown in linearity, (which implies a breakdown in the usual notion of inertial frame), or a breakdown of the existence of any notion of Hamiltonian/Lagrangian mechanics - any of which would then undermine the very notions of energy and momentum used to define the DSR energy-momentum 
transformations in the first place.

\section{A.2 Relative locality}

Additionally, there have recently been some speculations (and some significant disagreements) in the literature regarding non-local models based on so-called "relative locality" [20, 23, 24, 26, 153, 154]. Currently, these models are still being developed and investigated. Certainly, they very explicitly fall outside the framework we are considering in this article.

Roughly speaking, in relative locality models it is momentum space that is taken to be primary, with single-particle phase space being the tangent bundle to momentum space - the various tangent spaces [indexed by the 4-momentum] then correspond to logically distinct space-times indexed by the 4-momentum of the particle being observed. More generally, in multi-particle contexts these relative locality models appear to generalize/modify the notion of inertial frame in such a way that it depends not only on the state of motion of the observer, but also on the collection of 4-momenta of the various objects being observed.

\section{A.3 Some matrix identities}

Herein we collect some useful matrix identities of a purely technical nature. First note that

$$
(I-\boldsymbol{v} \otimes \boldsymbol{u})^{-1}=I+\sum_{n=1}^{\infty}(\boldsymbol{v} \otimes \boldsymbol{u})^{n}=I+(\boldsymbol{v} \otimes \boldsymbol{u}) \sum_{n=1}^{\infty}(\boldsymbol{u} \cdot \boldsymbol{v})^{n-1}=I+\frac{\boldsymbol{v} \otimes \boldsymbol{u}}{1-\boldsymbol{u} \cdot \boldsymbol{v}},
$$

with this particular derivation holding for $|\boldsymbol{u} \cdot \boldsymbol{v}|<1$, though the result itself

$$
(I-\boldsymbol{v} \otimes \boldsymbol{u})^{-1}=I+\frac{\boldsymbol{v} \otimes \boldsymbol{u}}{1-\boldsymbol{u} \cdot \boldsymbol{v}},
$$

holds for $\boldsymbol{u} \cdot \boldsymbol{v} \neq 1$, as can easily be verified by multiplying both sides of the equation above by $(I-\boldsymbol{v} \otimes \boldsymbol{u})$ and noting that $\operatorname{det}(I-\boldsymbol{v} \otimes \boldsymbol{u})=1-\boldsymbol{v} \cdot \boldsymbol{u}$. (The case $\boldsymbol{u} \cdot \boldsymbol{v}=1$ is the kinematic singularity alluded to previously.) Therefore

$$
\boldsymbol{u}^{T}(I-\boldsymbol{v} \otimes \boldsymbol{u})^{-1}=\boldsymbol{u}^{T}+\frac{(\boldsymbol{u} \cdot \boldsymbol{v}) \boldsymbol{u}^{T}}{1-\boldsymbol{u} \cdot \boldsymbol{v}}=\frac{\boldsymbol{u}^{T}}{1-\boldsymbol{u} \cdot \boldsymbol{v}}
$$

at least for $\boldsymbol{u} \cdot \boldsymbol{v} \neq 1$. Similarly

$$
(1-\boldsymbol{u} \cdot \boldsymbol{v})(I-\boldsymbol{v} \otimes \boldsymbol{u})^{-1}=(1-\boldsymbol{u} \cdot \boldsymbol{v}) I+\boldsymbol{v} \otimes \boldsymbol{u}
$$


for $\boldsymbol{u} \cdot \boldsymbol{v} \neq 1$. Secondly observe

$$
\begin{aligned}
(I-\boldsymbol{v} \otimes \boldsymbol{u})(I-\dot{\mathbf{x}} \otimes \boldsymbol{u})^{-1} & =(I-\boldsymbol{v} \otimes \boldsymbol{u})\left(I+\sum_{n=1}^{\infty}(\dot{\mathbf{x}} \otimes \boldsymbol{u})^{n}\right) \\
& =I-\boldsymbol{v} \otimes \boldsymbol{u}\left(\sum_{n=0}^{\infty}(\dot{\mathbf{x}} \cdot \boldsymbol{u})^{n}\right)+\dot{\mathbf{x}} \otimes \boldsymbol{u}\left(\sum_{n=0}^{\infty}(\dot{\mathbf{x}} \cdot \boldsymbol{u})^{n}\right) \\
& =I-\frac{\boldsymbol{v} \otimes \boldsymbol{u}}{1-\dot{\mathbf{x}} \cdot \boldsymbol{u}}+\frac{\dot{\mathbf{x}} \otimes \boldsymbol{u}}{1-\dot{\mathbf{x}} \cdot \boldsymbol{u}} \\
& =I+\frac{(\dot{\mathbf{x}}-\boldsymbol{v}) \otimes \boldsymbol{u}}{1-\dot{\mathbf{x}} \cdot \boldsymbol{u}}
\end{aligned}
$$

with this particular derivation holding for $|\dot{\mathbf{x}} \cdot \boldsymbol{u}|<1$, though the result itself holds for $\dot{\mathbf{x}} \cdot \boldsymbol{u} \neq 1$. Therefore, for $\dot{\mathbf{x}} \cdot \boldsymbol{u} \neq 1$, we have

$$
\begin{aligned}
(I-\boldsymbol{v} \otimes \boldsymbol{u})(I-\dot{\mathrm{x}} \otimes \boldsymbol{u})^{-1}(\dot{\mathrm{x}}-\boldsymbol{v}) & =\left(I+\frac{(\dot{\mathrm{x}}-\boldsymbol{v}) \otimes \boldsymbol{u}}{1-\dot{\mathrm{x}} \cdot \boldsymbol{u}}\right)(\dot{\mathrm{x}}-\boldsymbol{v}) \\
& =(\dot{\mathrm{x}}-\boldsymbol{v})+\frac{(\dot{\mathrm{x}}-\boldsymbol{v}) \boldsymbol{u} \cdot(\dot{\mathrm{x}}-\boldsymbol{v})}{1-\dot{\mathrm{x}} \cdot \boldsymbol{u}} \\
& =(\dot{\mathrm{x}}-\boldsymbol{v})\left\{\frac{1-\dot{\mathrm{x}} \cdot \boldsymbol{u}+\boldsymbol{u} \cdot(\dot{\mathrm{x}}-\boldsymbol{v})}{1-\dot{\mathrm{x}} \cdot \boldsymbol{u}}\right\} \\
& =(\dot{\mathrm{x}}-\boldsymbol{v})\left\{\frac{1-\boldsymbol{u} \cdot \boldsymbol{v}}{1-\dot{\mathrm{x}} \cdot \boldsymbol{u}}\right\}
\end{aligned}
$$

\section{A.4 Consistency of dynamics and kinematics}

Note that from Hamilton's equations we know $\dot{\mathbf{x}}=\partial H / \partial \boldsymbol{p}$, so to first order (which is all we require) $\Delta E=\dot{\mathrm{x}} \cdot \Delta \boldsymbol{p}$. Then from our discussion of the energy-momentum transformation laws, and specifically the fact that energy-momentum differences transform linearly, we have

$$
\dot{\mathbf{x}} \cdot \Delta \boldsymbol{p}=\gamma \dot{\overline{\mathbf{x}}} \cdot \Delta \overline{\boldsymbol{p}}+\Delta \overline{\boldsymbol{p}}^{T} \Sigma \boldsymbol{v}=\Delta \overline{\boldsymbol{p}} \cdot(\gamma \dot{\overline{\mathbf{x}}}+\Sigma \boldsymbol{v}),
$$

and

$$
\Delta \boldsymbol{p}=\gamma(\dot{\overline{\mathbf{x}}} \cdot \Delta \overline{\boldsymbol{p}}) \boldsymbol{u}+\Sigma^{T} \Delta \overline{\boldsymbol{p}}=\left(\gamma \boldsymbol{u} \otimes \dot{\overline{\mathbf{x}}}+\Sigma^{T}\right) \Delta \overline{\boldsymbol{p}} .
$$

But then, for arbitrary $\Delta \bar{p}$

$$
\left\{\dot{\mathbf{x}}^{T}\left(\gamma \boldsymbol{u} \otimes \dot{\overline{\mathbf{x}}}+\Sigma^{T}\right)-\left(\gamma \dot{\overline{\mathbf{x}}}^{T}+\Sigma \boldsymbol{v}^{T}\right)\right\} \Delta \overline{\boldsymbol{p}}=0,
$$

implying

$$
\dot{\mathbf{x}}^{T}\left(\gamma \boldsymbol{u} \otimes \dot{\overline{\mathbf{x}}}+\Sigma^{T}\right)=\left(\gamma \dot{\overline{\mathbf{x}}}^{T}+\boldsymbol{v}^{T} \Sigma^{T}\right) .
$$

That is

$$
(\gamma \dot{\overline{\mathbf{x}}} \otimes \boldsymbol{u}+\Sigma) \dot{\mathbf{x}}=(\gamma \dot{\overline{\mathbf{x}}}+\Sigma \boldsymbol{v})
$$


whence

$$
\dot{\mathbf{x}}=(\gamma \dot{\overline{\mathbf{x}}} \otimes \boldsymbol{u}+\Sigma)^{-1}(\gamma \dot{\overline{\mathbf{x}}}+\Sigma \boldsymbol{v}) .
$$

This is equivalent to the velocity transformation law we previously derived. (Note that $\dot{\overline{\mathbf{x}}}=\mathbf{0}$ implies $\dot{\mathbf{x}}=\boldsymbol{v}$, while $\dot{\mathbf{x}}=\mathbf{0}$ implies $\dot{\overline{\mathbf{x}}}=-\Sigma \boldsymbol{v} / \gamma$.)

To find the inverse transformations $\dot{\overline{\mathbf{x}}}(\dot{\mathbf{x}})$ is perhaps easier to start from the inverse transformations for energy and momentum

$$
\Delta E \rightarrow \Delta \bar{E}=\frac{\Delta E-\Delta \boldsymbol{p} \cdot \boldsymbol{v}}{\gamma(1-\boldsymbol{u} \cdot \boldsymbol{v})}
$$

and

$$
\Delta \boldsymbol{p} \rightarrow \Delta \overline{\boldsymbol{p}}=\left(\Sigma^{-1}\right)^{T}(I-\boldsymbol{u} \otimes \boldsymbol{v})^{-1}(\Delta \boldsymbol{p}-\Delta E \boldsymbol{u}) .
$$

The energy transformation equation implies

$$
\dot{\overline{\mathbf{x}}} \cdot \Delta \bar{p}=\frac{(\dot{\mathrm{x}}-\boldsymbol{v}) \cdot \Delta \boldsymbol{p}}{\gamma(1-\boldsymbol{u} \cdot \boldsymbol{v})},
$$

while the momentum transformation equation yields

$$
\begin{aligned}
\Delta \overline{\boldsymbol{p}} & =\left(\Sigma^{-1}\right)^{T}(I-\boldsymbol{u} \otimes \boldsymbol{v})^{-1}(\Delta \boldsymbol{p}-[\dot{\mathbf{x}} \cdot \Delta \boldsymbol{p}] \boldsymbol{u}) \\
& =\left(\Sigma^{-1}\right)^{T}(I-\boldsymbol{u} \otimes \boldsymbol{v})^{-1}(I-\boldsymbol{u} \otimes \dot{\mathbf{x}}) \Delta \boldsymbol{p} .
\end{aligned}
$$

But then

$$
\left\{\dot{\overline{\mathbf{x}}}^{T}\left(\Sigma^{-1}\right)^{T}(I-\boldsymbol{u} \otimes \boldsymbol{v})^{-1}(I-\boldsymbol{u} \otimes \dot{\mathbf{x}})-\frac{(\dot{\mathbf{x}}-\boldsymbol{v})^{T}}{\gamma(1-\boldsymbol{u} \cdot \boldsymbol{v})}\right\} \Delta \boldsymbol{p}=0,
$$

whence

$$
\dot{\overline{\mathbf{x}}}^{T}\left(\Sigma^{-1}\right)^{T}(I-\boldsymbol{u} \otimes \boldsymbol{v})^{-1}(I-\boldsymbol{u} \otimes \dot{\mathbf{x}})=\frac{(\dot{\mathbf{x}}-\boldsymbol{v})^{T}}{\gamma(1-\boldsymbol{u} \cdot \boldsymbol{v})} .
$$

Therefore

$$
(I-\dot{\mathbf{x}} \otimes \boldsymbol{u})(I-\boldsymbol{v} \otimes \boldsymbol{u})^{-1} \Sigma^{-1} \dot{\overline{\mathbf{x}}}=\frac{\dot{\mathbf{x}}-\boldsymbol{v}}{\gamma(1-\boldsymbol{u} \cdot \boldsymbol{v})},
$$

and we see

$$
\dot{\overline{\mathbf{x}}}=\Sigma(I-\boldsymbol{v} \otimes \boldsymbol{u})(I-\dot{\mathbf{x}} \otimes \boldsymbol{u})^{-1} \frac{(\dot{\mathbf{x}}-\boldsymbol{v})}{\gamma(1-\boldsymbol{u} \cdot \boldsymbol{v})} .
$$

But (see appendix A.3)

$$
(I-\boldsymbol{v} \otimes \boldsymbol{u})(I-\dot{\mathbf{x}} \otimes \boldsymbol{u})^{-1}=I+\frac{(\dot{\mathbf{x}}-\boldsymbol{v}) \otimes \boldsymbol{u}}{1-\dot{\mathbf{x}} \cdot \boldsymbol{u}} .
$$

Furthermore (see appendix A.3

$$
(I-\boldsymbol{v} \otimes \boldsymbol{u})(I-\dot{\mathbf{x}} \otimes \boldsymbol{u})^{-1}(\dot{\mathbf{x}}-\boldsymbol{v})=(\dot{\mathbf{x}}-\boldsymbol{v})\left\{\frac{1-\boldsymbol{u} \cdot \boldsymbol{v}}{1-\dot{\mathrm{x}} \cdot \boldsymbol{u}}\right\}
$$


So finally

$$
\dot{\overline{\mathbf{x}}}=\frac{\sum(\dot{\mathbf{x}}-\boldsymbol{v})}{\gamma(1-\boldsymbol{u} \cdot \dot{\mathbf{x}})}
$$

which is the 3 -velocity transformation law we had previously derived. (Note that $\dot{\overline{\mathbf{x}}}=\mathbf{0}$ implies $\dot{\mathbf{x}}=\boldsymbol{v}$, while $\dot{\mathbf{x}}=\mathbf{0}$ implies $\dot{\overline{\mathbf{x}}}=-\Sigma \boldsymbol{v} / \gamma$.) This verifies the internal consistency of the manner in which our Hamiltonian/Lagrangian mechanics interacts with the generic transformation laws between inertial frames. 
APPENDIX A. LORENTZ SYMMETRY BREAKING 


\section{Appendix B}

\section{Energy conditions}

In this appendix we shall present some of the background material that had been briefly alluded to in chapter 7. In particular we shall present the energy conditions and average energy conditions.

In general relativity, and more generically in relativistic classical field theories of gravitation, the energy conditions are applied to the matter content of the theory when it is either not possible or desirable to specify this content explicitly. They also provide the theory with a criterion to select the physically interesting solutions of the Einstein equation, and discard those that are unphysical. Mathematically speaking, the most apparent distinguishing feature of the energy conditions is that they are essentially restrictions on the eigenvalues and eigenvectors of the matter tensor.

In order to define the energy conditions one first considers the energy-momentum tensor $T^{a b}$ to be of Hawking-Ellis type I, so that in a suitable orthonormal frame it has components

$$
T^{a b}=\left[\begin{array}{cccc}
\rho & 0 & 0 & 0 \\
0 & p_{1} & 0 & 0 \\
0 & 0 & p_{2} & 0 \\
0 & 0 & 0 & p_{3}
\end{array}\right],
$$

These components are the energy density and the three principal pressures. This is the form of the energy-momentum for all observed fields with non-zero rest-mass and also for all zero rest-mass field except in the special case when it is of type II, see [141]. There exist three more types of stress energy tensor: 
1. Type II. This kind of energy-momentum tensor corresponds to the case of zero rest-mass fields when they represent radiation, all of which is travelling in the direction $E_{3}+E_{4}$, where $E_{3}+E_{4}$ is a double null energy eigenvector.

2. Type III. This is the special case in which the energy-momentum tensor has a triple null eigenvector $E_{3}+E_{4}$. There are no observed fields with such energy-momentum tensor.

3. Type $I V$. This is the general case in which the energy-momentum tensor has no timelike or null eigenvector. There are no observed fields with such an energy-momentum tensor.

The last three types of energy-momentum tensor are not used in the derivation of the energy conditions and for this reason we shall not specify any other of their properties.

Null energy condition (NEC). This condition is the assertion that for any null vector $k^{a}$

$$
T_{a b} k^{a} k^{b} \geq 0 .
$$

In terms of the principal pressures it becomes

$$
\forall j, \quad \rho+p_{j} \geq 0 .
$$

Weak energy condition (WEC). This condition is the assertion that for any timelike vector $V^{a}$

$$
T_{a b} V^{a} V^{b} \geq 0 .
$$

If this is true for any timeline vector, it will also by continuity imply the null energy condition. The physical meaning of this condition is that it forces the local energy density as measured by any timelike observer to be positive. In terms of the principal pressures it becomes

$$
\rho \geq 0 \quad \text { and } \quad \forall j, \quad \rho+p_{j} \geq 0 .
$$


Strong energy condition (SEC). This condition is the assertion that for any timelike vector $V^{a}$

$$
\left(T_{a b}-\frac{T}{2} g_{a b}\right) V^{a} V^{b} \geq 0
$$

where $T$ is the trace of the stress-energy tensor, $T=T_{a b} g^{a b}$. By continuity, the strong energy condition implies the null energy condition, but it does not imply, in general, the weak energy condition. In terms of the principal pressures we have

$$
T=-\rho+\sum_{j} p_{j}
$$

and the SEC becomes

$$
\forall j, \quad \rho+p_{j} \geq 0, \quad \text { and } \quad \rho+\sum_{j} p_{j} \geq 0
$$

Dominant energy condition (DEC). This condition is the assertion that for any timelike vector $V^{a}$

$$
T_{a b} V^{a} V^{b} \geq 0, \quad \text { and } \quad T_{a b} V^{a} \quad \text { is not spacelike. }
$$

This condition implies that the locally measured energy density is always positive, and that the energy flux is timelike or null. The dominant energy condition implies the weak energy condition, and hence the null energy condition, but does not necessarily imply the strong energy condition. In terms of the principal pressures it becomes

$$
\rho \geq 0 \quad \text { and } \quad \forall j, \quad p_{j} \in[-\rho,+\rho] .
$$

\section{B.1 Average energy conditions}

Each of the above-mentioned conditions has an averaged version, in which the properties noted above are to hold only on average along the flow-lines of some appropriate vector field $(\Gamma)$.

Average null energy condition (ANEC). This condition is said to hold on a null curve $\Gamma$ if

$$
\int_{\Gamma} T_{a b} k^{a} k^{b} d \lambda \geq 0
$$


where $\lambda$ is a generalised affine parameterisation of the null curve and $k^{a}$ is the corresponding tangent vector. There are some technical points:

1. If $\Gamma$ is a null geodesic then the generalised affine parameter specialises to the ordinary affine parameter, see for example [141].

2. Arbitrary parameterisations are not allowed, otherwise the ANEC would be equivalent to the ordinary NEC.

3. Because of the multiplicative arbitrariness of the generalised affine parameter and ordinary affine parameter, it is only meaningful to define the ANEC integral up to an overall positive multiplicative constant.

In order to phrase this condition in terms of the principal pressures, one can use a normalisation function $\xi$, and direction cosines $\cos \psi_{i}$, defined by

$$
k^{a} \equiv \xi\left(1 ; \cos \psi_{i}\right)
$$

The ANEC can then be written as

$$
\int_{\Gamma}\left(\rho+\sum_{j} \cos ^{2} \psi_{j} p_{j}\right) \xi^{2} d \lambda \geq 0 .
$$

Usually one typically requires the ANEC to hold on some suitable class $\Gamma$ of inextendible null geodesics.

Average weak energy condition (AWEC). This condition is said to hold on a timelike curve $\Gamma$ if

$$
\int_{\Gamma} T_{a b} V^{a} V^{b} d s \geq 0
$$

Here $s$ denotes the proper time parameterisation of the curve $\Gamma$, whose corresponding tangent vector is $V^{a}$. With the choice of $s$ as the proper time one has

$$
V^{a}=\gamma\left(1 ; \beta \cos \psi_{j}\right)
$$

Hence

$$
\int_{\Gamma} \gamma^{2}\left(\rho+\beta^{2} \sum_{j} \cos ^{2} \psi_{j} p_{j}\right) d s \geq 0 .
$$

When applied, one usually requires the AWEC to hold on some suitable class $\Gamma$ of inextensible timelike geodesics. Some technical points are: 
1. If this class of timelike geodesics is suitably large, its boundary $\partial \Gamma$ may contain limit points (limit curves) consisting of null geodesics. In this sense, AWEC (on the set $\Gamma$ ) can be said to imply ANEC (on the null geodesics in the set $\partial \Gamma)$.

2. In a general space-time there may be null geodesics that are not obtained as the limit of any sequence of timelike geodesics. In general, AWEC and ANEC are independent conditions.

Average strong energy condition (ASEC). This condition is said to hold on a timelike curve $\Gamma$ if

$$
\int_{\Gamma}\left(T_{a b} V^{a} V^{b}+\frac{1}{2} T\right) d s \geq 0 .
$$

In terms of the principal pressure this gives the constraint

$$
\int_{\Gamma}\left\{\gamma^{2}\left(\rho+\beta^{2} \sum_{j} \cos ^{2} \psi_{j} p_{j}\right)-\frac{1}{2} \rho+\frac{1}{2} \sum_{j} p_{j}\right\} d s \geq 0 .
$$

If $\beta \rightarrow 1$, then $\gamma \rightarrow \infty$, while $\gamma d s \rightarrow d \lambda$ and $d s \rightarrow 0$. In this limit ASEC reduces to the ANEC - up to an irrelevant infinite multiplicative factor.

\section{B.2 Some applications}

The energy conditions, in one form or another, are used in the various classical singularity theorems and theorems of classical black hole thermodynamics. We shall report here just a few examples.

- The Penrose singularity theorem invokes the weak energy condition, see [141].

- The Hawking-Penrose singularity theorem invokes the strong energy condition, see [141] and [291].

- The proof the zeroth law of black hole thermodynamics (the constancy of surface gravity over the event horizon) relies on the dominant energy condition [291].

- The proof of the second law of black hole thermodynamics (the area increase theorem) uses the null energy condition [291].

For other applications and an exhaustive list of all the known violations refer to [141] and [275. 
APPENDIX B. ENERGY CONDITIONS 


\section{Appendix C}

\section{Generalised second law}

The generalised second law was introduced by Jacob Bekenstein in 1973 [63], after it was noticed that, if one restricts the attention to the matter outside the black hole, one can decrease its entropy when falling inside the black hole itself, violating the second law of thermodynamics - that states that the total entropy of matter in the universe never decreases.

Indeed it is now established, at least at a theoretical level, that there exists an analogy between the laws of thermodynamics and the laws of black hole physics, derived from classical general relativity. This analogy can be considered an equivalence if one takes into account quantum effects, identifying energy $E$ and mass $M$, temperature $T$ and surface gravity $\kappa$, entropy $S$ and even horizon area $A$ (up to some multiplicative factors).

In order to avoid such a violation of the second law, Bekenstein defined the generalised entropy

$$
S^{\prime}=S+\frac{1}{4} k \frac{c^{2} A}{G \hbar}
$$

where $S$ is the entropy of the matter falling into the black hole, $k$ is Boltzmann's constant, $G$ the gravitational constant and $A$ the horizon area. The fact that a decrease in $S$ seems always to be compensated by an increase in $A$ and, similarly, a decrease in $A$ seems always to be compensated by an increase in $\mathrm{S}$, suggests that the generalised second law

$$
\delta S^{\prime} \geq 0
$$

In the case described in chapter 7, we are considering an energy-momentum tensor $T^{a b}$ that satisfies the null energy condition (NEC). This guarantees the positivity of the total Clausius entropy flux, that includes the entropy of the bulk $S_{\mathcal{B}}$ and the 
entropy of the bifurcate null surface, implying that the total entropy of the systems obeys

$$
\delta S_{\text {Clausius }} \geq 0 \text {. }
$$




\section{Appendix D}

\section{Jensen's inequality and Shannon entropy bounds}

In this appendix we shall give a brief introduction to Jensen's inequality and its application to the cases that lead to inequalities 8.3 and 8.17 in chapter 8 .

\section{D.1 Definition of Jensen's inequality}

Jensen's inequality [171] relates the value of a convex/concave function of an integral to the integral of the convex/concave function, and it generalises the statement that the secant line of a convex function lies above the graph of the function - Jensen's inequality for two points. In the finite case, if $f(x)$ is a convex function, numbers $x_{1}, x_{2}, \ldots, x_{n}$ in its domain, and positive weights $a_{i}$, Jensen's inequality can be stated as

$$
f\left(\frac{\sum a_{i} x_{i}}{\sum a_{j}}\right) \leq \frac{\sum a_{i} f\left(x_{i}\right)}{\sum a_{j}}
$$

and the inequality is reversed if the function is concave, as it is in our case:

$$
f\left(\frac{\sum a_{i} x_{i}}{\sum a_{j}}\right) \geq \frac{\sum a_{i} f\left(x_{i}\right)}{\sum a_{j}}
$$

In the following subsections we shall carefully explain how to obtain the inequalities 8.3 and (8.17).

\section{D.1.1 Shannon entropy bounds}

Regarding inequality 8.3 for the case with a finite number of states with probability different from zero we have that: 


$$
S=-\sum_{n} p_{n} \ln p_{n}=\sum_{n} p_{n} \ln \left(\frac{1}{p_{n}}\right) .
$$

If we now consider that logarithm function is a concave function, we can apply eq. (D.2), with $\sum_{i} a_{i}=\sum p_{i}=1$, and obtain:

$$
S \leq \ln \left(\sum_{n} \frac{p_{n}}{p_{n}}\right)=\ln N,
$$

that is the inequality we were looking for.

Regarding inequality (8.17), we know that for the function $f(x)=x \ln x, f^{\prime \prime}=$ $1 / x>0$ for $x>0$, therefore $f(x)$ is always convex for the interval of $x$ we are considering. Applying the inequality (D.1) to our case

$$
\sum_{i=1}^{2} p_{i}\left(x_{i} \ln x_{i}\right) \geq \sum_{i} p_{i} x_{i} \ln \left(\sum_{i} p_{i} x_{i}\right),
$$

where $x_{i}=\tilde{x}, \tilde{y}$ and $p_{i}=\frac{a}{a+b}, \frac{b}{a+b}$, therefore leading to

$$
\left(\frac{a \tilde{x}}{a+b}+\frac{b \tilde{y}}{a+b}\right) \ln \left(\frac{a \tilde{x}}{a+b}+\frac{b \tilde{y}}{a+b}\right)=\left(\frac{a \tilde{x}+b \tilde{y}}{a+b}\right) \ln \left(\frac{a \tilde{x}+b \tilde{y}}{a+b}\right)
$$

that is the inequality we were looking for. 


\section{Appendix E}

\section{Bose-Einstein condensate and analogue space-time}

In this appendix we shall explain in more detail some of the characteristics of the analogue model of space-time that we have used in section 9.3 such as definition of an effective metric for a relativistic BEC and the validity of this approximation when the system undergoes an acceleration. For a review in the subject refer to the Living Review by Barceló, Liberati, and Visser [56] and references therein.

\section{E.1 Effective space-time for a relativistic Bose-Einstein condensate}

The Lagrangian density of a Bose-Einstein condensate on a space-time metric $g_{a b}$ trapped by an external potential $V\left(x^{\mu}\right)$ is given by [107],

$$
\hat{\mathcal{L}}=\sqrt{-g} g^{a b} \partial_{a} \Phi^{\dagger} \partial_{b} \Phi-\left(\frac{m^{2} c^{2}}{\hbar^{2}}+V\left(x^{\mu}\right)\right) \Phi^{\dagger} \Phi-U\left(\Phi^{\dagger} \Phi ; \lambda_{i}\right) .
$$

where $c$ is the speed of light, $\hbar$ Planck's constant and $g=\operatorname{det} g_{a b}$. The atomic field $\Phi$ consists of $N$ atoms of mass $m$ that interact with each other through $U\left(\hat{\phi}^{\dagger} \hat{\phi} ; \lambda_{i}\right)$. The interaction strengths $\lambda_{i}$ can in principle depend on the coordinates $x^{\mu}$ of the background space-time. In the regime below the critical temperature $T_{c}$, the atomic field can be approximated by $\Phi=\Phi_{0}(1+\Pi)$, where $\Phi_{0}$ is a classical background field and $\Pi$ is a quantum field corresponding to fluctuations known as phonons. In this regime, the background field obeys the non-linear Klein-Gordon equation

$$
\square_{g} \Phi_{0}-\left(\frac{m^{2} c^{2}}{\hbar^{2}}+V\left(x^{\mu}\right)\right) \Phi_{0}-U^{\prime}\left(\rho ; \lambda_{i}\right) \Phi_{0}=0
$$


where $\rho:=\Phi_{0}^{*} \Phi_{0}$ is the background density and

$$
\square_{g}:=\frac{1}{\sqrt{-g}} \partial_{a} \sqrt{-g} \partial^{a},
$$

is the d'Alembertian operator. The superscript in $U^{\prime}$ denotes the derivatives with respect to $\rho$. Equation (E.2) reduces to the standard Gross-Pitaevskii equation in the Newtonian limit $c^{2} \rightarrow \infty$ [107]. On the other hand, the quantum fluctuations $\Pi$ obey the field equation

$$
\square_{g} \Pi+2 g^{a b}\left(\partial_{a} \ln \Phi_{0}\right) \partial_{b} \Pi-\rho U^{\prime \prime}\left(\rho ; \lambda_{i}\right)=0 .
$$

Writing $\Phi_{0}=\sqrt{\rho} e^{i \theta}$ we define the generalized kinetic operators as

$$
T_{\rho} \equiv-\frac{\hbar^{2}}{2 m}\left(\square_{g}+g^{a b} \partial_{a} \ln \rho \partial_{b}\right),
$$

and the effective speed of phonon propagation $c_{0}^{2} \equiv \frac{\hbar^{2}}{2 m^{2}} \rho U^{\prime \prime}\left(\rho ; \lambda_{i}\right)$, and the fourvelocity vectors $u^{a} \equiv \frac{\hbar}{m} g^{a b} \partial_{b} \theta$. We can then rewrite the equation as

$$
\left\{\left[i \hbar u^{a} \partial_{a}+T^{\rho}\right] \frac{1}{c_{0}^{2}}\left[-i \hbar u^{b} \partial_{b}+T^{\rho}\right]-\frac{\hbar^{2}}{\rho} g^{a b} \partial_{a} \rho \partial_{b}\right\} \Pi=0 .
$$

The quantity $T_{\rho}$ can be neglected when the dispersion relation for the perturbations is $\omega^{2}=c_{s}^{2} k^{2}$ and if one is working in the eikonal approximation [107]. That is when the background quantities vary slowly in space and time on scales comparable with the wavelength and the period of the perturbations, respectively [107]. This assumption is equivalent to neglecting the quantum pressure term in the GrossPitaevskii equation obtained in the Newtonian limit. In this case equation E.6 becomes the Klein-Gordon equation

$$
\square_{\mathfrak{g}} \Pi=\frac{1}{\sqrt{-\mathfrak{g}}} \partial_{a} \sqrt{-\mathfrak{g}} \partial^{a} \Pi=0,
$$

where the effective metric $\mathfrak{g}_{a b}$ is defined as

$$
\mathfrak{g}_{a b}=\frac{\rho}{\sqrt{1-u_{d} u^{d} / c_{0}^{2}}}\left[g_{a b}\left(1-\frac{u_{d} u^{d}}{c_{0}^{2}}\right)+\left(\frac{u_{a} u_{b}}{c_{0}^{2}}\right)\right] .
$$

By defining the four-velocity $v^{a} \equiv \frac{c}{\|u\|} u^{a}$ and the scalar speed of sound

$$
c_{s}^{2}=\frac{c^{2} c_{0}^{2} /\|u\|^{2}}{1+c_{0}^{2} /\|u\|^{2}}
$$

the effective metric can be written as

$$
\mathfrak{g}_{\mathfrak{a} \mathfrak{b}}=\frac{c}{c_{s}} \frac{n_{0}}{\varrho_{0}+p_{0}}\left[g_{a b}+\left(1-\frac{c_{s}^{2}}{c^{2}}\right) v_{a} v_{b}\right]
$$

The conformal factor in the last equation $(\mathrm{E} .10)$ can be found by considering the hydrodynamical description for a BEC [107]. 


\section{E.2 Effective metric and accelerating BEC}

One important point one needs to take into account is the fact that, when the BEC undergoes acceleration, the background density can become inhomogeneous. This implies that it is not possible to neglect the generalised kinetic operator in equation (E.6) that describes the motion of the quantum fluctuations $\Pi(x)$. Therefore it is not possible to use the analogue model approximation and describe the motion of the quantum fluctuations with quantum field theory in a curved space-time.

Fortunately, in the acceleration regimes we consider $a \simeq 10^{-3} \mathrm{~m} / \mathrm{s}^{2}$, these effects are negligible. Indeed, mimicking the acceleration by an external potential of the form $V(x)=m \cdot a \cdot x$ [209], with $m$ the atomic mass, and $x$ a measure of the BEC displacement due to the acceleration, we find that the term associated to the quantum pressure $T_{\rho}$ can be safely neglected as long as

$$
\frac{\partial_{x} \rho}{\rho} \simeq \frac{h}{L} \ll \frac{m c}{\hbar} .
$$

Here $h$ is not the Planck constant but $h=\frac{a L}{c^{2}}{ }_{2}$, and $L$ is the length of the BEC trap. Given the values for $a$ and $c_{s}$ mentioned above, we have that $h / L \simeq 10^{3} \mathrm{~m}^{-1}$ while $m c / \hbar$ is larger than $10^{15} \mathrm{~m}^{-1}$. Therefore the condition is satisfied and we can still define an effective metric. 
APPENDIX E. BEC AND ANALOGUE SPACE-TIME 


\section{Appendix F}

\section{Quantum information tools}

In this appendix we shall present a few quantum information tools that we have used in chapter 9 , section 9.4 to describe the evolution of the quantum states and the entanglement when one of the two BEC systems undergoes a constant acceleration while the other continues on an inertial trajectory.

\section{F.1 Covariance matrix formalism}

The covariance matrix is a general tool used in probabilistic theory and statistics. Also known as the dispersion matrix or variance-covariance matrix, it is a matrix whose element $(i, j)$ is the covariance between the $i$-th and $j$-th elements of a random vector, each element of which is a scalar random variable, either with a finite number of observed empirical values or with a finite or infinite number of potential values specified by a theoretical joint probability distribution of all the random variables.

\section{General definition}

If we define the random vector as

$$
\mathbf{X}=\left(\begin{array}{c}
X_{1} \\
\vdots \\
X_{n}
\end{array}\right)
$$

where $X_{i}$ are random variables, each with finite variance, then we can define the $(i, j)$ element of the covariance matrix $\sigma$ as

$$
\sigma_{i j}:=\mathrm{E}\left[\left(X_{i}-\mu_{i}\right)\left(X_{j}-\mu_{j}\right)\right]
$$

The values 


$$
\mu_{i}=\mathrm{E}\left(X_{i}\right)
$$

are the expected value of the $i$-th entry in the vector $\mathbf{X}$.

In the area of interest of quantum information, the covariance matrix is very useful when considering Gaussian states - that is states with Gaussian characteristic functions and quasi-probability distributions. Such distributions are completely described by the mean and the variance, i.e. they are determined by the vector of first moments

$$
\mathbf{X}:=\left(\hat{q}_{1}, \hat{p}_{1}, \hat{q}_{2}, \hat{p}_{2}, \ldots, \hat{q}_{n}, \hat{p}_{n}\right)^{T},
$$

and the real, symmetric covariance matrix $\sigma$, that in this case has components

$$
\sigma_{i j}:=\left\langle X_{i} X_{j}+X_{j} X_{i}\right\rangle_{\rho}-2\left\langle X_{i}\right\rangle_{\rho}\left\langle X_{j}\right\rangle_{\rho} .
$$

Here $\langle.\rangle_{\rho}$ is the expectation value in the state $\rho$ and $\hat{q}_{j}$ and $\hat{p}_{j}$ are the quadrature phase operators defined as

$$
\begin{aligned}
\hat{q}_{j} & :=\frac{1}{\sqrt{2}}\left(a_{j}+a_{j}^{\dagger}\right) \\
\hat{p}_{j} & :=\frac{-i}{\sqrt{2}}\left(a_{j}-a_{j}^{\dagger}\right) .
\end{aligned}
$$

The operators $a_{i}$ and $a_{i}^{\dagger}$ are the usual annihilation and creation operators for the mode $i$. The expectation value $\langle.\rangle_{\rho}$ is defined in terms of the density matrix as

$$
\langle A\rangle_{\rho}=\operatorname{Tr}(A \rho)
$$

with $A$ being an operator.

\section{F.2 Squeezed coherent states}

A squeezed coherent state is any state in a Hilbert space such that the uncertainty principle is saturated. That is, the product of the corresponding two operators takes on its minimum value:

$$
\Delta X \Delta P=\frac{\hbar}{2}
$$

The simplest such state is the ground state $|0\rangle$ of the quantum harmonic oscillator. Indeed, we shall use the quantum harmonic oscillator to give the basic idea of 
squeezing. In particular let us consider the vacuum state wave function in position basis and momentum basis

$$
\psi_{0}=\frac{1}{\pi^{1 / 4}} e^{-X^{2} / 2}, \quad \tilde{\psi}_{0}=\frac{1}{\pi^{1 / 4}} e^{-P^{2} / 2} .
$$

(Here we are assuming that $\hbar=1$ ) The variance of the position and momentum observables in the vacuum state is $\left\langle 0\left|\Delta X^{2}\right| 0\right\rangle=\left\langle 0\left|\Delta P^{2}\right| 0\right\rangle=1 / 2$. The squeezevacuum state $\left|s q_{r}\right\rangle$, with squeezing parameter $r$, is obtain from the vacuum state by means of a scaling transformation, that for the wave function is

$$
\psi_{r}(X)=\frac{\sqrt{r}}{\pi^{1 / 4}} e^{-(r X)^{2} / 2}, \quad \tilde{\psi}_{r}(P)=\frac{1}{\pi^{1 / 4} \sqrt{r}} e^{-(P / r)^{2} / 2} .
$$

In this state, the variances of the two canonical observables become

$$
\left\langle\Delta X^{2}\right\rangle=\frac{1}{2 r^{2}}, \quad\left\langle\Delta P^{2}\right\rangle=\frac{r^{2}}{2} .
$$

If $r>1$, the position variance is below that of the vacuum state, so $\left|s q_{r}\right\rangle$ is positionsqueezed; for $r<1$ the state is momentum-squeezed. Therefore, the measurements of the squeezed observable on each copy of $\left|s q_{r}\right\rangle$ will exhibit less a variance than if performed on multiple copies of the vacuum state.

In general, a state of a single harmonic oscillator exhibits (quadrature) squeezing if the variance of the position, momentum, or any other quadrature $\hat{X}_{\theta}=$ $\hat{X} \cos \theta+\hat{P} \sin \theta$ - with $\theta$ a real angle known as quadrature angle - in that state exhibits variance below $1 / 2$. In accordance with the uncertainty principle, both position and momentum observables, or in fact any two quadratures associated with orthogonal angles, cannot be squeezed at the same time. For instance, for position and momentum we still have $\left\langle\Delta X^{2}\right\rangle\left\langle\Delta P^{2}\right\rangle=1 / 4$ for the vacuum state.

In general, a single-mode squeezed state $|\alpha, \xi\rangle$ is generated by a single-mode squeezing operator $S(\xi)$, defined as

$$
S(\xi)=\exp \left(\frac{\xi^{*}}{2} a^{2}-\frac{\xi}{2} a^{\dagger 2}\right)
$$

with $\xi=r e^{i \theta}$ a complex number, $r \geq 0,0 \leq \theta \leq 2 \pi$ in the following way

$$
|\alpha, \xi\rangle=D(\alpha) S(\xi)|0\rangle,
$$

and $D(\alpha)=\exp \left(\alpha a^{\dagger}-\alpha^{*} a\right)$ is the coherent state displacement operator with $\alpha=$ $|\alpha| e^{i \phi}$.

The annihilation operator is transformed by the squeezing operator as follows 


$$
S^{-1}(\xi) a S(\xi)=a \cosh r-a^{\dagger} e^{i \theta} \sinh r .
$$

The variance of the position and momentum becomes

$$
\begin{aligned}
\left\langle\Delta X^{2}\right\rangle_{s q} & =\frac{1}{2}\left(e^{-2 r} \cos ^{2} \frac{\theta}{2}+e^{2 r} \sin ^{2} \frac{\theta}{2}\right) \\
\left\langle\Delta P^{2}\right\rangle_{s q} & =\frac{1}{2}\left(e^{-2 r} \sin ^{2} \frac{\theta}{2}+e^{2 r} \cos ^{2} \frac{\theta}{2}\right),
\end{aligned}
$$

therefore

$$
\left\langle\Delta X^{2}\right\rangle_{s q}\left\langle\Delta P^{2}\right\rangle_{s q}=\frac{1}{4}\left(1+\sin ^{2} \theta \sinh ^{2} 2 r\right) \geq \frac{1}{4} .
$$

When $\theta=0$ we recover the minimum uncertainty relation and, depending on the sign of $r$, either $\left\langle\Delta X^{2}\right\rangle_{s q}$ or $\left\langle\Delta P^{2}\right\rangle_{s q}$ can be smaller than $1 / 2$.

In a similar way, it is possible to generate two-mode squeezed state as

$$
\left|\alpha_{1}, \alpha_{2}, \xi\right\rangle=D_{1}\left(\alpha_{1}\right) D_{2}\left(\alpha_{2}\right) S_{1,2}(\xi)|0\rangle
$$

with $D_{1}$ and $D_{2}$ the displacement operators, and $S_{1,2}(\xi)$ the two-mode squeezing operator defined as

$$
S_{1,2}=\exp \left(\xi^{*} a_{1} a_{2}-\xi a_{1}^{\dagger} a_{2}^{\dagger}\right)
$$

1,2 represent the two particles. The creation and annihilation operators for 1,2 transform accordingly to (F.14).

The generalised quadrature operators are defined by

$$
\begin{aligned}
& X_{1,2}=\frac{1}{2^{3 / 2}}\left(a_{1}+a_{1}^{\dagger}+a_{2}+a_{2}^{\dagger}\right), \\
& X_{1,2}=\frac{1}{2^{3 / 2}}\left(a_{1}-a_{1}^{\dagger}+a_{2}-a_{2}^{\dagger}\right) .
\end{aligned}
$$

The variances are

$$
\begin{aligned}
\left\langle\Delta X_{1,2}^{2}\right\rangle_{s q} & =\frac{1}{2}\left(e^{-2 r} \cos ^{2} \frac{\theta}{2}+e^{2 r} \sin ^{2} \frac{\theta}{2}\right), \\
\left\langle\Delta P_{1,2}^{2}\right\rangle_{s q} & =\frac{1}{2}\left(e^{-2 r} \sin ^{2} \frac{\theta}{2}+e^{2 r} \cos ^{2} \frac{\theta}{2}\right) .
\end{aligned}
$$


Therefore, the variance of one of the quadrature operators can decrease while the other one simultaneously increases to satisfy the uncertainty principle. Similarly as before, for $\theta=0$ we recover the minimum uncertainty relation and, depending on the sign of $r$, either $\left\langle\Delta X_{1,2}^{2}\right\rangle_{s q}$ or $\left\langle\Delta P_{1,2}^{2}\right\rangle_{s q}$ can be smaller than $1 / 2$.

\section{F.3 Negativity as a measure of entanglement}

Negativity is one of the possible measures of entanglement of a mixed state. Generally speaking the requirements that one imposes on entanglement measures $E(\rho)$ are

\section{Definition: Entanglement measure:}

An entanglement measure $E(\rho)$ is a map from density operators $\rho$ to the nonnegative real numbers $E(\rho) \in \mathbb{R}_{0}^{+}$that satisfies:

(i) $E(\rho)=0$ for all separable states.

(ii) $E(\rho)$ is non-increasing under local operations and classical communication.

An extensive review of the various available entanglement measures, entanglement monotones, and their connections can be found in [228, 273].

\section{Definition: Negativity}

Negativity is based on the positive partial transpose criterion (PPT), or PeresHorodecki criterion, a criterion established by A. Peres and M., P. and R. Horodecki to detect entanglement [146, 224]. The PPT criterion states the following

- A bipartite state $\rho_{A B} \in \mathbb{C}_{2} \otimes \mathbb{C}_{2}$ or $\mathbb{C}_{2} \otimes \mathbb{C}_{3}$ is separable if, and only if, the partial transposition of $\rho_{A B}$ is positive,i.e.

$$
\rho_{A B}^{T_{B}}=\left(\mathbb{1} \otimes T_{B}\right) \rho_{A B} \geq 0 .
$$

This is based on the fact that, since the transposition preserves the positivity of operators, one can show that that separable states remain positive under partial transposition, see [146, 224, 228].

Going into more detail, we first write a general bipartite mixed state $\rho \in \mathcal{H}_{A B}$ in terms of local bases $|m\rangle \in \mathcal{H}_{A}$ and $|n\rangle \in \mathcal{H}_{B}$ as 


$$
\rho=\sum_{m, m^{\prime}, n n^{\prime}} \rho_{m, n, m^{\prime}, n^{\prime}}|m\rangle\left\langle m^{\prime}|\otimes| n\right\rangle\left\langle n^{\prime}\right| .
$$

The partial transposition is obtained by exchanging the indices of the operators on one of the subspaces

$$
\rho^{T_{B}}=\left(\mathbb{1} \otimes T_{B}\right) \rho=\sum_{m, m^{\prime}, n n^{\prime}} \rho_{m, n, m^{\prime}, n^{\prime}}|m\rangle\left\langle m^{\prime}|\otimes| n^{\prime}\right\rangle\langle n| .
$$

In terms of these quantities the negativity $N(\rho)$ of a bipartite state $\rho$ is defined as

$$
N(\rho):=\frac{1}{2} \sum_{i}\left(\left|\lambda_{i}\right|-\lambda_{i}\right)
$$

where $\lambda_{i} \in\left[-\frac{1}{2}, 1\right]$ are the eigenvalues of $\rho^{T_{B}}$. The negativity can also be defined in terms of the trace norm $\|.\|_{1}$ as

$$
N(\rho):=\frac{1}{2}\left(\left\|\rho^{T_{B}}\right\|_{1}-1\right),
$$

with $\|\rho\|_{1}=\operatorname{Tr} \sqrt{\rho^{\dagger} \rho}$. 


\title{
Appendix G
}

\section{Publications not included in this thesis}

\section{G.1 Massive gravity from bimetric gravity}

\author{
Valentina Baccetti, Prado Martin Moruno, Matt Visser
}

Electronic preprint ArXiv: 1205.2158 [gr-qc]

Published in Classical and Quantum Gravity 30 (2013) 015004

DOI: $10.1088 / 0264-9381 / 30 / 1 / 015004$

We discuss the subtle relationship between massive gravity and bimetric gravity, focusing particularly on the manner in which massive gravity may be viewed as a suitable limit of bimetric gravity. The limiting procedure is more delicate than currently appreciated. Specifically, this limiting procedure should not unnecessarily constrain the background metric, which must be externally specified by the theory of massive gravity itself. The fact that in bimetric theories one always has two sets of metric equations of motion continues to have an effect even in the massive gravity limit, leading to additional constraints besides the one set of equations of motion naively expected. Thus, since solutions of bimetric gravity in the limit of vanishing kinetic term are also solutions of massive gravity, but the contrary statement is not necessarily true, there is not complete continuity in the parameter space of the theory. In particular, we study the massive cosmological solutions which are continuous in the parameter space, showing that many interesting cosmologies belong to this class. 


\title{
G.2 Null Energy Condition violations in bimetric gravity
}

\author{
Valentina Baccetti, Prado Martin Moruno, Matt Visser \\ Electronic preprint ArXiv: 1206.3814 [gr-qc] \\ Published in JHEP 1208 (2012) 148 \\ DOI: $10.1007 / J H E P 08(2012) 148$
}

We consider the effective stress-energy tensors for the foreground and background sectors in ghost-free bimetric gravity. By considering the symmetries of the theory, we show that the foreground and background null energy conditions (NECs) are strongly anti-correlated. In particular, the NECs can only be simultaneously fulfilled when they saturate, corresponding to foreground and background cosmological constants. In all other situations, either the foreground or the background is subject to a NEC-violating contribution to the total stress-energy.

\section{G.3 Gordon and Kerr-Schild ansatze in massive and bimetric gravity}

\author{
Valentina Baccetti, Prado Martin Moruno, Matt Visser \\ Electronic preprint ArXiv: 1206.4720 [gr-qc] \\ Published in JHEP 1208 (2012) 108 \\ DOI: $10.1007 / J H E P 08(2012) 108$
}

We develop the "generalized Gordon ansatz" for the ghost-free versions of both massive and bimetric gravity, an ansatz which is general enough to include almost all space-times commonly considered to be physically interesting, and restricted enough to greatly simplify calculations. The ansatz allows explicit calculation of the matrix square root $\gamma=\sqrt{g^{-1} f}$ appearing as a central feature of the ghost-free analysis. In particular, this ansatz automatically allows us to write the effective stress-energy tensor as that corresponding to a perfect fluid. A qualitatively similar "generalized Kerr-Schild ansatz" can also be easily considered, now leading to an effective stress-energy tensor that corresponds to a null fluid. Cosmological implications are 
considered, as are consequences for black hole physics. Finally we have a few words to say concerning the null energy condition in the framework provided by these ansatze.

\title{
G.4 Massive gravity as a limit of bimetric gravity
}

\author{
Prado Martin Moruno, Valentina Baccetti, Matt Visser \\ Electronic preprint ArXiv: 1302.2687 [gr-qc]
}

13th Marcel Grossmann Meeting, Conference Proceedings: C12-07-01.1

Massive gravity may be viewed as a suitable limit of bimetric gravity. The limiting procedure can lead to an interesting interplay between the "background" and "foreground" metrics in a cosmological context. The fact that in bimetric theories one always has two sets of metric equations of motion continues to have an effect even in the massive gravity limit. Thus, solutions of bimetric gravity in the limit of vanishing kinetic term are also solutions of massive gravity, but the contrary statement is not necessarily true.

\section{G.5 Inertial frames without the relativity principle: breaking Lorentz symmetry}

\author{
Valentina Baccetti, Kyle Tate, Matt Visser \\ Electronic preprint ArXiv: 1302.5989 [gr-qc]
}

13th Marcel Grossmann Meeting, Conference Proceedings: C12-07-01.1

We investigate inertial frames in the absence of Lorentz invariance, reconsidering the usual group structure implied by the relativity principle. We abandon the relativity principle, discarding the group structure for the transformations between inertial frames, while requiring these transformations to be at least linear (to preserve homogeneity). In theories with a preferred frame (aether), the set of transformations between inertial frames forms a groupoid/pseudogroup instead of a group, a characteristic essential to evading the von Ignatowsky theorems. In order to understand the dynamics, we also demonstrate that the transformation rules for energy and momentum are in general affine. We finally focus on one specific and compelling model implementing a minimalist violation of Lorentz invariance. 


\section{Appendix $\mathbf{H}$}

\section{Curriculum vitæ}

\section{H.1 Education}

Bachelor's thesis

- Title: Correlation Function for a Catalog of Infrared Galaxies

- Supervisor: Prof. Enzo Branchini

- Date and Place: October 2004, Dipartimento di Fisica, "E.Amaldi", Università "RomaTre"

- Final Grade: 110/110 cum laude (first class honours degree)

Master's thesis

- Title: The particle interpretation of $N=1$ supersymmetric spin foams

- Supervisors: Dr. James Ryan (Perimeter Institute), Prof. Orlando Ragnisco (RomaTre)

- Date and Place: September 2010, Dipartimento di Fisica, "E.Amaldi", Università "RomaTre" (Thesis work undertaken at Perimeter Institute)

- Final Grade: 110/110 cum laude (first class honours degree) 


\section{PhD thesis}

- Title: Phenomena at the border between quantum physics and general relativity

- Supervisor: Prof. Matt Visser

- Date and Place: April 2014 (thesis submission), School of Mathematics, Statistics and Operations Research, Victoria University of Wellington

\section{H.2 Scholarship and prizes}

- Victoria PhD Scholarship for doctoral study at Victoria University of Welling-ton, December 2010.

- Best talk in the applied mathematics session at the New Zealand Mathematics and Statistics Postgraduate Conference, Auckland, November 2012.

- Hartle award for one of the best student presentations at the 20th International Conference of General Relativity and Gravitation (GR20), Warsaw, July 2013.

- Irene Pestov Memorial Scholarship for 2014 that aims to assist female students in the area of mathematical sciences or geosciences to complete their MSc or PhD thesis at Victoria University of Wellington, November 2013.

- Kerr Prize (shared) for the best student presentation at the Seventh Australasian Conference on General Relativity and Gravitation (ACGRG7), Hamilton Island, Queensland, December 2013.

\section{H.3 Other publications}

- The Redshift-Space Two Point Correlation Function of ELAIS-S1 Galaxies, V. D'Elia, E. Branchini, F. La Franca, V. Baccetti, I. Matute, F. Pozzi, C. Gruppioni, Mon. Not. Roy. Astron. Soc. 359 (2005), 1077-1082.

- The particle interpretation of $N=1$ supersymmetric spin foams, V. Baccetti, E. R. Livine, J. P. Ryan, Class. Quant. Grav., 27 (2010), 225022. 


\section{Bibliography}

[1] Abreu, G., Barceló, C., And Visser, M. Entropy bounds in terms of the $w$ parameter. JHEP 1112 (2011), 092.

[2] Abreu, G., And Visser, M. Tolman mass, generalized surface gravity, and entropy bounds. Phys. Rev. Lett. 105 (2010), 041302.

[3] Abreu, G., and Visser, M. Entropy bounds for uncollapsed matter. J. Phys. Conf. Ser 314 (2011), 012035.

[4] Abreu, G., And Visser, M. Entropy bounds for uncollapsed rotating bodies. JHEP 1103 (2011), 056.

[5] Adam, T., And et al. Opera Collaboration. Measurement of the neutrino velocity with the OPERA detector in the CNGS beam. JHEP 1210 (2012), 093.

[6] Adamson, P., And et al. Minos Collaboration. Measurement of neutrino velocity with the MINOS detectors and NuMI neutrino beam. Phys. Rev. D 76 (2007), 072005.

[7] Adesso, G., And Illuminati, F. Gaussian measures of entanglement versus negativities: Ordering of two-mode Gaussian states. Phys. Rev. A 72 (Sep 2005), 032334.

[8] Ahluwalia, D. V. Operational indistinguishability of doubly special relativities from special relativity. ArXiv:gr-qc/0212128, 2002.

[9] Alexandre, J. Lifshitz-type Quantum Field Theories in Particle Physics. Int. J. Mod. Phys. A26 (2011), 4523.

[10] Alexandre, J., Ellis, J., And Mavromatos, N. E. On the Possibility of Superluminal Neutrino Propagation. Phys.Lett. B706 (2012), 456-461. 
[11] Aloisio, R., A.Galante, Grillo, A. F., Luzio, E., and Mendez, F. Approaching space time through velocity in doubly special relativity. Phys. Rev. D 70 (2004), 125012.

[12] Aloisio, R., Blasi, P., Ghia, P. L., and Grillo, A. F. Probing the structure of space-time with cosmic rays. Phys. Rev. D 62 (2000), 053010.

[13] Aloisio, R., Galante, A., Grillo, A. F., Luzio, E., and Mendez, F. A Note on DSR-like approach to space-time. Phys. Lett. B 610 (2005), 101.

[14] Alsing, P. M., And Fuentes, I. Observer dependent entanglement. Class.Quant.Grav. 29 (2012), 224001.

[15] Ambjorn, J., Dasgupta, A., Jurkiewicz, J., And Loll, R. A Lorentzian cure for Euclidean troubles. Nuclear Physics B Proceedings Supplements 106 (Mar. 2002), 977-979.

[16] Ambjorn, J., Jurkiewicz, J., And Loll, R. Reconstructing the universe. Phys.Rev. D72 (2005), 064014.

[17] Ambjorn, J., Jurkiewicz, J., And Loll, R. The Universe from scratch. Contemp.Phys. 47 (2006), 103-117.

[18] Amelino-Camelia, G. Doubly special relativity. Nature 418 (2002), 34.

[19] Amelino-Camelia, G. Relativity in space-times with short distance structure governed by an observer independent (Planckian) length scale. Int. J. Mod. Phys. D 11 (2002), 35.

[20] Amelino-Camelia, G. On the fate of Lorentz symmetry in relative-locality momentum spaces. ArXiv:1110.5081 [hep-th], 2011.

[21] Amelino-Camelia, G. Particle-dependent deformations of Lorentz symmetry. Symmetry \& (2012), 344.

[22] Amelino-Camelia, G., Ellis, J. R., Mavromatos, N. E., Nanopoulos, D. V., AND SARKAR, S. Tests of quantum gravity from observations of gamma-ray bursts. Nature 393 (1998), 763.

[23] Amelino-Camelia, G., Freidel, L., Kowalski-Glikman, J., And Smolin, L. Relative locality: A deepening of the relativity principle. Gen. Rel. Grav. 43 (2011), 2547. 
[24] Amelino-Camelia, G., Freidel, L., Kowalski-Glikman, J., And Smolin, L. The principle of relative locality. Phys. Rev. D 84 (2011), 084010.

[25] Amelino-Camelia, G., Gubitosi, G., Loret, N., Mercati, F., Rosati, G., ET AL. OPERA-reassessing data on the energy dependence of the speed of neutrinos. Int.J.Mod.Phys. D20 (2011), 2623-2640.

[26] Amelino-Camelia, G., Matassa, M., Mercati, F., and Rosati, G. Taming nonlocality in theories with Planck-scale deformed Lorentz symmetry. Phys. Rev. Lett. 106 (2011), 071301.

[27] Amelino-Camelia, G., And Piran, T. Planck scale deformation of Lorentz symmetry as a solution to the UHECR and the TeV gamma paradoxes. Phys. Rev. D 64 (2001), 03600.

[28] Anselmi, D. Renormalization of Lorentz violating theories. In Prepared for 4th Meeting on CPT and Lorentz Symmetry, Bloomington, Indiana (8-11 Aug 2007).

[29] Anselmi, D. Weighted scale invariant quantum field theories. JHEP 0802 (2008), 051.

[30] Anselmi, D. Weighted power counting and Lorentz violating gauge theories. I: General properties. Annals Phys. 324 (2009), 874.

[31] Anselmi, D. Weighted power counting and Lorentz violating gauge theories. II: Classification. Annals Phys. 324 (2009), 1058.

[32] Anselmi, D. Weighted power counting, neutrino masses and Lorentz violating extensions of the Standard Model. Phys. Rev. D 79 (2009), 025017.

[33] Anselmi, D. Standard model without elementary scalars and high energy Lorentz violation. Eur. Phys. J. C 65 (2010), 523.

[34] Anselmi, D. Renormalization and Lorentz symmetry violation. PoS C LAQG08 (2011), 010.

[35] Anselmi, D., and Buttazzo, D. Distance between quantum field theories as a measure of Lorentz violation. Phys. Rev. D 84 (2011), 036012.

[36] Anselmi, D., And Ciuffoli, E. Renormalization of high-energy Lorentz violating four Fermion models. Phys. Rev. D 81 (2010), 085043. 
[37] Anselmi, D., And Ciuffoli, E. Low-energy phenomenology of scalarless standard-model extensions with high-energy Lorentz violation. Phys. Rev. D 83 (2011), 056005.

[38] Anselmi, D., And Halat, M. Renormalization of Lorentz violating theories. Phys. Rev. D 76 (2007), 125011.

[39] Anselmi, D., and Taiuti, M. Renormalization of high-energy Lorentz violating QED. Phys. Rev. D 81 (2010), 085042.

[40] Anselmi, D., And Taiuti, M. Vacuum Cherenkov radiation in quantum electrodynamics with high-energy Lorentz violation. Phys. Rev. D 83 (2011), 2011.

[41] Armitage, S. E., Stras, L., Bonin, G. R. R. R. E., and Zee, R. E. In IWSCFF-2013-08-05, 7th Int. Workshop on Satellite Constellations and Formation Flying, Lisbon, Portugal (2013).

[42] Arnowitt, R., Deser, S., And Misner, C. Republication of: The dynamics of general relativity. General Relativity and Gravitation 40, 9 (2008), 1997-2027.

[43] Ash, R. B. Information Theory. Dover, New York, 1990.

[44] Ashtekar, A. Gravity and the quantum. New J.Phys. 7 (2005), 198.

[45] Baccetti, V., Tate, K., and Visser, M. Inertial frames without the relativity principle. JHEP 1205 (2012), 119.

[46] Baccetti, V., Tate, K., And Visser, M. Lorentz violating kinematics: Threshold theorems. JHEP 1203 (2012), 087.

[47] Baccetti, V., And Visser, M. Infinite Shannon entropy. JSTAT - Journal of Statistical Mechanics: Theory and Experiment 1304 (2013), P04010.

[48] Baccetti, V., And Visser, M. Clausius entropy for arbitrary bifurcate null surfaces. Class. Quant. Grav. 31 (2014), 035009.

[49] Baez, J., And Muniain, J. P. Gauge Fields, Knots and Gravity. World Scientific, 1994.

[50] Baez, J. C. Spin foam models. Class.Quant.Grav. 15 (1998), 1827-1858. 
[51] Barbado, L. C., And Visser, M. Unruh-DeWitt detector event rate for trajectories with time-dependent acceleration. Phys. Rev. D 86 (2012), 084011.

[52] Barceló, C., Liberati, S., Sonego, S., And M.Visser. Hawking-like radiation does not require a trapped region. Phys. Rev. Lett. $9^{77}$ (2006), 171301.

[53] Barceló, C., Liberati, S., Sonego, S., And Visser, M. Hawking-like radiation from evolving black holes and compact horizonless objects. JHEP 1102 (2011), 003.

[54] Barceló, C., Liberati, S., Sonego, S., and Visser, M. Minimal conditions for the existence of a Hawking-like flux. Phys. Rev. D 83 (2011), 041501.

[55] Barceló, C., Liberati, S., And Visser, M. Analog gravity from BoseEinstein condensates. Class. Quant. Grav. 18 (2001), 1137.

[56] Barcelo, C., Liberati, S., And Visser, M. Analogue gravity. Living Rev. Rel. 8 (2005), 12.

[57] Barceló, C., And Visser, M. Twilight for the energy conditions? Int. J. Mod. Phys. D 11 (2002), 1553.

[58] Bardeen, J. M., Carter, B., and Hawking, S. W. The four laws of black hole mechanics. Communications in Mathematical Physics 31, 2 (1973), 161-170.

[59] Bear, D., Stoner, R. E., Walsworth, R. L., Kostelecky, V. A., And LANE, C. D. Limit on Lorentz and CPT violation of the neutron using a two-species noble-gas maser. Phys. Rev. Lett 85 (2000), 5038.

[60] Becker, K., Becker, M., And Schwarz, J. H. String theory and M-theory: A modern introduction. Cambridge University Press, 2007.

[61] Beig, R., And Chrusciel, P. T. Stationary black holes. ArXiv: grqc/0502041, 2005.

[62] Bekenstein, J. Black holes and entropy. Phys. Rev. D 7 (1973), 2333-2346.

[63] Bekenstein, J. D. Generalized second law of thermodynamics in black-hole physics. Phys. Rev. D 9 (Jun 1974), 3292-3300.

[64] Bekenstein, J. D. A Universal Upper Bound on the Entropy to Energy Ratio for Bounded Systems. Phys. Rev. D 23 (1981), 287. 
[65] Bender, C. M., Brody, D. C., And Meister, B. J. Unusual quantum states: non-locality, entropy, Maxwell's demon and fractals. Proc. R. Soc. A 461 (2005), 733-753.

[66] Bergmann, P., And Komar, A. The phase space formulation of general relativity and approaces toward its canonical quantization. General Relativity and Gravitation vol 1, One Hundred Years after the Birth of Albert Einstein. Plenum, New York, 1981.

[67] Berzi, V., And Gorini, V. Reciprocity principle and the Lorentz transformations. J. Math. Phys. 10 (1969), 1518-1524.

[68] Berzi, V., And Gorini, V. On space-time, reference frames and the structure of relativity groups. Annales Poincare Phys. Theor. 16 (1972), 1-22.

[69] Bi, X.-J., Yin, P.-F., Yu, Z.-H., And Yuan, Q. Constraints and tests of the OPERA superluminal neutrinos. Phys.Rev.Lett. 107 (2011), 241802.

[70] Birrell, N. D., And Davies, P. C. W. Quantum fields in curved space. Cambridge: University Press, 1982.

[71] Bombelli, L., Koul, R. K., Lee, J., and Sorkin, R. D. A quantum source of entropy for black holes. Phys. Rev. D 34 (1986), 373.

[72] Bonato, C., Tomaello, A., Deppo, V. D., Naletto, G., and VilLORESI, P. Feasibility of satellite quantum key distribution. New Journal of Physics 11, 4 (Apr. 2009), 045017.

[73] Bruschi, D. E., Fris, N., Fuentes, I., And Weinfurtner, S. On the robustness of entanglement in analogue gravity systems. New Journal of Physics 15, 11 (Nov. 2013), 113016.

[74] Bruschi, D. E., Fuentes, I., And Louko, J. Voyage to Alpha Centauri: Entanglement degradation of cavity modes due to motion. Phys. Rev. D 85 (Mar 2012), 061701.

[75] Bruschi, D. E., Sabín, C., White, A., Baccetti, V., Oi, D. K. L., ET AL. Testing the effects of gravity and motion on quantum entanglement in space-based experiments. New Journal of Physics 16 (2014), 053041.

[76] Cacciapaglia, G., Deandrea, A., and Panizzi, L. Superluminal neutrinos in long baseline experiments and SN1987a. JHEP 1111 (2011), 137. 
[77] Calmet, X., Hossenfelder, S., and Percacci, R. Deformed special relativity from asymptotically safe gravity. Phys. Rev. D 82 (2010), 124024.

[78] Carlen, E. Trace inequalities and quantum entropy: An introductory course. In Entropy and the Quantum: A school on analytic and functional inequalities with applications (Tucson, Arizona, March 16-20 2009).

[79] Carmona, J., and Cortes, J. Constraints from neutrino decay on superluminal velocities. ArXiv: 1110.0430 [hep-ph], 2011.

[80] Chadha, S., and B.Nielsen, H. Lorentz invariance as a low-energy phenomenon. Nucl. Phys. B 217 (1983), 125.

[81] Chandrasekhar, S. An introduction to the study of stellar structure. Dover, 1957.

[82] Chirco, G., Eling, C., And Liberati, S. The universal viscosity to entropy density ratio from entanglement. Phys. Rev. D 82 (2010), 024010.

[83] Chirco, G., Eling, C., And Liberati, S. Higher curvature gravity and the holographic fluid dual to flat spacetime. JHEP 1108 (2011), 009.

[84] Chirco, G., Eling, C., and S.Liberati. Reversible and irreversible spacetime thermodynamics for general Brans-Dicke theories. Phys. Rev. D 83 (2011), 024032.

[85] Chirco, G., And S.Liberati. Non-equilibrium thermodynamics of spacetime: The role of gravitational dissipation. Phys. Rev. D 81 (2010), 024016.

[86] Clausius, R. On the application of the theorem of the equivalence of transformations to interior work. Communicated to the Naturforschende Gesellschaft of Zurich, January 27, 1862; published in the Vierteljahrschrift of this Society, vol. vii. p. 48; in Poggendorff's Annalen, May 1862, vol. cxvi. p. 73; in the Philosophical Magazine, S. 4. vol. xxiv. pp. 81, 201; and in the Journal des Mathematiques of Paris, S. 2. vol. vii. p. 209., 1862.

[87] Cohen, A. G., And Glashow, S. L. Pair creation constrains superluminal neutrino propagation. Phys. Rev. Lett $10^{7}$ (2011), 181803.

[88] Coleman, S. R., and Glashow, S. L. Cosmic ray and neutrino tests of special relativity. Phys.Lett. B405 (1997), 249-252. 
[89] Coleman, S. R., and Glashow, S. L. High-energy tests of Lorentz invariance. Phys.Rev. D59 (1999), 116008.

[90] Coleman, S. R., Preskill, J., And Wilczek, F. Quantum hair on black holes. Nucl. Phys. B 378 (1992), 175.

[91] Colladay, D., And Kostelecky, V. A. Lorentz-violating extension of the standard model. Phys. Rev. D 58 (1998), 116002.

[92] Cowsik, R., Nussinov, S., And Sarkar, U. Superluminal neutrinos at OPERA confront pion decay kinematics. Phys. Rev. Lett. 107 (2011), 251801.

[93] DAss, N. D. H. OPERA, SN1987a and energy dependence of superluminal neutrino velocity. ArXiv: 1110.0351 [hep-ph], 2011.

[94] Daszkiewicz, M., Imilkowska, K., and Kowalski-Glikman, J. Velocity of particles in doubly special relativity. Phys. Lett. A 323 (2004), 345.

[95] De Montigny, M., and Rousseaux, G. On some applications of Galilean electrodynamics of moving bodies. American Journal of Physics 75 (2007), 984-992.

[96] Deb, B., And Agarwal, G. S. Entanglement of two distant Bose-Einstein condensates by detection of Bragg-scattered photons. Phys. Rev. A 78 (Jul 2008), 013639.

[97] Deriglazov, A. A. Doubly special relativity in position space starting from the conformal group. Phys. Lett. B 603 (2004), 124.

[98] DeWitt, B. S. Covariant Quantum Geometrodynamics. In Magic Without Magic: John Archibald Wheeler (1972), J. R. Klauder, Ed., W. H. Freeman, San Fransisco.

[99] Doukas, J., Lin, S., Hu, B., And Mann, R. B. Unruh Effect under Non-equilibrium conditions: Oscillatory motion of an Unruh-DeWitt detector. JHEP 1311 (2013), 119.

[100] Du, S., Squires, M. B., Imai, Y., Czaia, L., Saravanan, R. A., Bright, V., Reichel, J., Hänsch, T. W., And Anderson, D. Z. Atom-chip BoseEinstein condensation in a portable vacuum cell. Phys. Rev. A 70 (Nov 2004), 053606 . 
[101] Duff, M. Covariant quantization. In Quantum Gravity, An Oxford Symposium (1975), C. J. Isham, R. Penrose, and D. W. Sciama, Eds., Clarendon Press, Oxford.

[102] Dvali, G., And Vikman, A. Price for Environmental Neutrino-Superluminality. JHEP 1202 (2012), 134.

[103] Dyson, F. Missed Opportunities. Bulletin of the American Mathematical Society 78 (1972), 635-652.

[104] Einstein, A. Naeherungsweise Integration der Feldgleichungen der Gravitation. Sitzungsberichte der Preussischen Akademie der Wissenschaften zu Berlin (June 22 1916).

[105] Eisert, J., Cramer, M., and Plenio, M. B. Colloquium: Area laws for the entanglement entropy. Reviews of Modern Physics 82 (2010), 277-306.

[106] Eisert, J., Simon, C., And Plenio, M. B. On the quantification of entanglement in infinite-dimensional quantum systems. Journal of Physics A 35 (2002), 3911-3923.

[107] Fagnocchi, S., Finazzi, S., Liberati, S., Kormos, M., and TrombetTONI, A. Relativistic Bose-Einstein condensates: a new system for analogue models of gravity. New J.Phys. 12 (2010), 095012.

[108] Fewster, C. J. A General worldline quantum inequality. Class. Quant. Grav. 17 (2000), 1897.

[109] Fewster, C. J., And Roman, T. A. Null energy conditions in quantum field theory. Phys. Rev. D 67 (2003), 044003.

[110] Fewster, C. J., And Smith, C. J. Absolute quantum energy inequalities in curved spacetime. Annales Henri Poincare 9 (2008), 425.

[111] Fewster, C. J., And Teo, E. Quantum inequalities and 'quantum interest' as eigenvalue problems. Phys. Rev. D 61 (2000), 084012.

[112] Fiola, T. M., Preskill, J., Strominger, A., And Trivedi, S. P. Black hole thermodynamics and information loss in two-dimensions. Phys. Rev. D 50 (1994), 3987.

[113] Flanagan, E. E., And Wald, R. M. Does back reaction enforce the averaged null energy condition in semiclassical gravity? Phys. Rev. D 54 (1996), 6233 . 
[114] Fock, V. The Theory of Space, Time and Gravitation, Revised Second Edition. Pergamon Press, 1964.

[115] Ford, L. H. Constraints on negative energy fluxes. Phys. Rev. D 43 (1991), 3972 .

[116] Ford, L. H., And A.Roman, T. The quantum interest conjecture. Phys. Rev. D 60 (1999), 104018.

[117] Ford, L. H., Pfenning, M. J., and Roman, T. A. Quantum inequalities and singular negative energy densities. Phys. Rev. D 57 (1998), 4839.

[118] Ford, L. H., And Roman, T. A. Averaged energy conditions and quantum inequalities. Phys. Rev. D 51 (1995), 4277.

[119] Ford, L. H., And Roman, T. A. Restrictions on negative energy density in flat spacetime. Phys. Rev. D 55 (1997), 2082.

[120] Frank, P., And Rothe, H. Über die transformation der raumzeitkoordinaten von ruhenden auf bewegte systeme. Ann. Phys. 34 (1911), 825-853.

[121] Frank, P., ANd Rothe, H. Zur herleitung der Lorentz transformation. Phys. Zeitsch. 13 (1912), 750-753.

[122] Friedman, J. L., Schleich, K., And M.Witt, D. Topological censorship. Phys. Rev. Lett 71 (1993), 1486.

[123] Friss, N., And Fuentes, I. Entanglement generation in relativistic quantum fields. Journal of Modern Optics 60 (Jan. 2013), 22-27.

[124] Fris, N., Lee, A. R., Bruschi, D. E., And Louko, J. Kinematic entanglement degradation of fermionic cavity modes. Phys. Rev. D85 (2012), 025012 .

[125] Fris, N., Lee, A. R., Truong, K., Sabin, C., Solano, E., et al. Relativistic quantum teleportation with superconducting circuits. Phys. Rev. Lett. 110, 11 (2013), 113602.

[126] Gambini, R., And Pullin, J. Nonstandard optics from quantum space-time. Phys. Rev. D 59 (1999), 124021.

[127] Gaunt, A. L., Schmidutz, T. F., Gotlibovych, I., Smith, R. P., And HadzibABic, Z. Bose-Einstein condensation of atoms in a uniform potential. Phys. Rev. Lett. 110 (May 2013), 200406. 
[128] Ghosh, S. A Lagrangian for DSR particle and the role of noncommutativity. Phys. Rev. D 74 (2006), 084019.

[129] Girelli, F., Konopka, T., Kowalski-Glikman, J., and Livine, E. R. The Free particle in deformed special relativity. Phys. Rev. D 73 (2006), 045009 .

[130] Girelli, F., And Livine, E. R. Physics of deformed special relativity: Relativity principle revisited. ArXiv:0412004 [gr-qc], 2004.

[131] Giudice, G. F., Sibiryakov, S., And Strumia, A. Interpreting OperA results on superluminal neutrino. Nucl.Phys. B861 (2012), 1-16.

[132] Gorini, V. Linear kinematical groups. Commun. Math. Phys. 21 (1971), 150-163.

[133] Gorini, V., And ZeccA, A. Isotropy of space. J. Math. Phys. 11 (1970), $2226-2230$.

[134] Gromov, N. A., And Kuratov, V. V. Quantum kinematics. ArXiv: 0410086 [hep-th], 2004.

[135] Grumiller, D., Kummer, W., And Vassilevich, D. V. A Note on the triviality of Kappa deformations of gravity. Ukr. J. Phys. 48 (2003), 329.

[136] Gubser, S. S. Superluminal neutrinos and extra dimensions: Constraints from the null energy condition. Phys. Lett. B705 (2011), 279-281.

[137] Hansel, W., Hommelhoff, P., Hansch, T. W., and Reichel, J. BoseEinstein condensation on a microelectronic chip. Nature 413 (2001), 498-501.

[138] Hardy, G., And Riesz, M. The general theory of Dirichlet's series, vol. Republished by Cornell University Library 1991. Cambridge University Press, England, 1915.

[139] Hardy, G. H. A course in pure mathematics, vol. reprinted 2006. Cambridge University Press, England, 1908.

[140] Hardy, G. H., Littlewood, J. E., And Polya, G. Inequalities, vol. reprinted 1997. Cambridge University Press, England, 1934.

[141] Hawking, S., And Ellis, G. The Large scale structure of space-time. Cambridge University Press, 1973. 
[142] Hawking, S. W. Particle creation by black holes. Communications in Mathematical Physics 43, 3 (1975), 199-220.

[143] Hinterleitner, F. Canonical DSR. Phys. Rev. D 71 (2005), 025016.

[144] Hohmann, W. Die Erreichbarkeit der Himmelskorper. Verlag Oldenburg, Munich, 1925.

[145] Hohmann, W. The attainability of heavenly bodies. NASA technical translation, 1925.

[146] Horodecki, M., Horodecki, P., And Horodecki, R. Separability of mixed states: necessary and sufficient conditions. Physics Letters A 223 (1996), 1-8.

[147] Hossenfelder, S. Deformed special relativity in position space. Phys. Lett. B 649 (2007), 310 .

[148] Hossenfelder, S. Multi-particle states in deformed special relativity. Phys. Rev. D 75 (2007), 105005.

[149] Hossenfelder, S. The box-problem in deformed special relativity. ArXiv:0912.0090 [gr-qc], 2009.

[150] Hossenfelder, S. Bounds on an energy-dependent and observer-independent speed of light from violations of locality. Phys. Rev. Lett. 104 (2010), 140402.

[151] Hossenfelder, S. Comment on arXiv:1007.0718 by Lee Smolin. ArXiv:1008.1312 [gr-qc], 2010.

[152] Hossenfelder, S. Comments on nonlocality in deformed special relativity, in reply to arXiv:1004.0664 by Lee Smolin and arXiv:1004.0575 by Jacob et al. ArXiv:1005.0535 [gr-qc], 2010.

[153] Hossenfelder, S. Reply to arXiv:1006.2126 by Giovanni Amelino-Camelia et al. ArXiv:1006.4587 [gr-qc], 2010.

[154] Hossenfelder, S. Comment on arXiv:1104.2019, 'Relative locality and the soccer ball problem,' by Amelino-Camelia et al. Phys.Rev. D88 (2013), 028701.

[155] Houlrik, J. M., And Rousseaux, G. 'Nonrelativistic' kinematics: Particles or waves? ArXiv: 1005.1762v1 [gen-ph], 2010. 
[156] HořAva, P. Quantum Gravity at a Lifshitz Point. Phys.Rev. D79 (2009), 084008.

[157] Jacobson, T. Thermodynamics of space-time: The Einstein equation of state. Phys. Rev. Lett. 75 (1995), 1260.

[158] Jacobson, T., Liberati, S., And Mattingly, D. TeV astrophysics constraints on Planck scale Lorentz violation. Phys.Rev. D66 (2002), 081302.

[159] Jacobson, T., Liberati, S., And Mattingly, D. A Strong astrophysical constraint on the violation of special relativity by quantum gravity. Nature 424 (2003), 1019-1021.

[160] Jacobson, T., Liberati, S., And Mattingly, D. Comments on 'Improved limit on quantum space-time modifications of Lorentz symmetry from observations of gamma-ray blazars. ArXiv: 0303001 [gr-qc], 2003.

[161] Jacobson, T., Liberati, S., and Mattingly, D. Threshold effects and Planck scale Lorentz violation: Combined constraints from high-energy astrophysics. Phys.Rev. D67 (2003), 124011.

[162] Jacobson, T., Liberati, S., And Mattingly, D. Astrophysical bounds on Planck suppressed Lorentz violation. Lect.Notes Phys. 669 (2005), 101-130.

[163] Jacobson, T., Liberati, S., And Mattingly, D. Quantum gravity phenomenology and Lorentz violation. Springer Proc.Phys. 98 (2005), 83-98.

[164] Jacobson, T., Liberati, S., And Mattingly, D. Lorentz violation at high energy: Concepts, phenomena and astrophysical constraints. Annals Phys. 321 (2006), 150-196.

[165] Jacobson, T. A., Liberati, S., Mattingly, D., And Stecker, F. New limits on Planck scale Lorentz violation in QED. Phys. Rev. Lett. 93 (2004), 021101.

[166] Jammer, M. Some foundational problems in the special theory of relativity in Problems in the Foundations of Physics. G. Toraldo di Francia, Amsterdam, North-Holland, 1979, pages 202-236.

[167] Jaskula, J.-C., Partridge, G. B., Bonneau, M., Lopes, R., Ruaudel, J., Boiron, D., And Westbrook, C. I. Acoustic analog to the dynamical casimir effect in a Bose-Einstein condensate. Phys. Rev. Lett. 109 (Nov 2012), 220401. 
[168] Jaynes, E. T. Information Theory and Statistical Mechanics. Physical Review Series II 106 (1957), 620-630.

[169] Jaynes, E. T. Information Theory and Statistical Mechanics II. Physical Review Series II 108 (1957), 171-190.

[170] Jaynes, E. T. Probability Theory: The Logic of Science. Cambridge University Press, 2003.

[171] Jensen, J. L. W. V. Sur les fonctions convexes et les inégalités entre les valeurs moyennes. Acta Mathematica 30 (1906), 175-193.

[172] Judes, S., AND Visser, M. Conservation laws in 'Doubly special relativity'. Phys. Rev. D68 (2003), 045001.

[173] Kehagias, A. Relativistic Superluminal Neutrinos. ArXiv: 1109.6312 [hep$\mathrm{ph}], 2011$.

[174] KIfune, T. Invariance violation extends the cosmic ray horizon? Astrophys. J. 518 (1999), L21.

[175] Klinkhamer, F. Superluminal muon-neutrino velocity from a Fermi-pointsplitting model of Lorentz violation. ArXiv: 1109.5671 [hep-ph], 2011.

[176] Klinkhamer, F. R., And Volovik, G. E. Superluminal neutrino and spontaneous breaking of Lorentz invariance. Pisma Zh. Eksp. Teor. Fiz. 94 (2011), 731.

[177] Komar, A. Quantization program for general relativity. Plenum, New Tork, 1970.

[178] Konopka, T., Markopoulou, F., and Severini, S. Quantum graphity: A model of emergent locality. Phys.Rev. D77 (2008), 104029.

[179] Konopka, T., Markopoulou, F., and Smolin, L. Quantum Graphity. ArXiv: 0611197 [ hep-th], 2006.

[180] Kostelecky, V. A. Gravity, Lorentz violation, and the standard model. Phys. Rev. D 69 (2004), 105009.

[181] Kostelecky, V. A., And Lane, C. D. Constraints on Lorentz violation from clock-comparison experiments. Phys. Rev. D 60 (60), 116010. 
[182] Kostelecky, V. A., And Lehner, R. Stability, causality, and Lorentz and CPT violation. Phys. Rev. D 63 (2001), 065008.

[183] Kostelecky, V. A., And Mewes, M. Cosmological constraints on Lorentz violation in electrodynamics. Phys. Rev. Lett. 87 (2001), 251304.

[184] Kostelecky, V. A., And Mewes, M. Signals for Lorentz violation in electrodynamics. Phys. Rev. D 66 (2002), 056005.

[185] Kostelecky, V. A., And Mewes, M. Lorentz and CPT violation in neutrinos. Phys. Rev. D 69 (2004), 016005.

[186] Kostelecky, V. A., And Samuel, S. Spontaneous Breaking of Lorentz Symmetry in String Theory. Phys. Rev. D 39 (1989), 683.

[187] Kothawala, D., and Padmanabhan, T. Response of Unruh-DeWitt detector with time-dependent acceleration. Phys. Lett. B 690 (2010), 20.

[188] Kunang, L.-M., Chen, Z.-B., and Pan, J.-W. Generation of entangled coherent states for distant Bose-Einstein condensates via electromagnetically induced transparency. Phys. Rev. A 76 (Nov 2007), 052324.

[189] Kuchar, K. Canonical methods of quantization. In Quantum gravity 2, A second Oxford Symposium (1980), C. J. Isham, R. Penrose, and D. Sciama, Eds., Clarendon Press, Oxford.

[190] Kunn, T. S. The structure of scientific revolutions. University of Chicago Press., 1962.

[191] Kumar, T., Bhattacherjee, A. B., Verma, P., and ManMohan. Entangling two Bose-Einstein condensates in a double cavity system. Journal of Physics B Atomic Molecular Physics 44, 6 (Mar. 2011), 065302.

[192] Lalan, V. Sur les postulats qui sont à la base des cinématiques. Bull. Soc. Math. France 65 (1937), 83-99.

[193] Langer, S. A., Sethna, J. P., And Grannan, E. R. Nonequilibrium entropy and entropy distributions. Physical Review B 41 (1990), 2261-2278.

[194] Lee, A. R., and Kalotas, T. M. Lorentz transformations from the first postulate. American Journal of Physics 43 (1975), 434-437.

[195] LÉvy-Leblond, J. M. Une nouvelle limite non-relativiste du groupe de Poincaré. Annales de l'Institut Henri Poincaré A3 (1965), 1-12. 
[196] LÉvy-LeBlond, J. M. One more derivation of the Lorentz transformation. American Journal of Physics 44 (1976), 1-13.

[197] Liberati, S., Girelli, F., And Sindoni, L. Analogue models for emergent gravity. CNUM: C08-09-22.7, 2009.

[198] Liberati, S., Jacobson, T., and Mattingly, D. High-energy constraints on Lorentz symmetry violations. ArXiv: 0110094 [hep-ph], 2001.

[199] Liberati, S., Sonego, S., And Visser, M. Faster than $c$ signals, special relativity, and causality. Annals Phys. 298 (2002), 167.

[200] Liberati, S., Sonego, S., And Visser, M. Interpreting doubly special relativity as a modified theory of measurement. Phys. Rev. D71 (2005), 045001.

[201] Ling, A., AND Oi, D. K. L. Small photon-entangling quantum systems (SPEQS) for LEO satellites. In Proc. Int. Conf. Space Opt. Systems and Applications 2012 (Ajaccio, Corsica, France, 2012).

[202] Loll, R. Discrete lorentzian quantum gravity. Nucl. Phys. Proc. Suppl. 94 (2001), 96-107.

[203] Louko, J., And Satz, A. How often does the Unruh-DeWitt detector click? Regularisation by a spatial profile. Class. Quant. Grav. 23 (2006), 6321.

[204] Lugiato, L. A., And Gorini, V. On the structure of relativity groups. J. Math. Phys. 13 (1972), 665-671.

[205] Maccione, L., Liberati, S., and Mattingly, D. M. Violations of Lorentz invariance in the neutrino sector after OPERA. JCAP 1303 (2013), 039.

[206] Mansouri, R., And Sexl, R. U. A test theory of special relativity: I. Simultaneity and clock synchronization. Gen. Relativ. Gravit. 8 (1977), 497513.

[207] Martín-Moruno, P., And Visser, M. Classical and quantum flux energy conditions for quantum vacuum states. Physical Review D88 (2013), 061701R.

[208] Martín-Moruno, P., And Visser, M. Semiclassical energy conditions for quantum vacuum states. JHEP 1309 (2013), 050.

[209] Marzlin, K.-P., And Zhang, W. Acceleration of quasi-particle modes in Bose-Einstein condensates. Physics Letters A 248 (Nov. 1998), 290-294. 
[210] Mattingly, D. Modern tests of Lorentz invariance. Living Rev.Rel. 8 (2005), 5.

[211] Mattingly, D., Jacobson, T., And Liberati, S. Threshold configurations in the presence of Lorentz violating dispersion relations. Phys.Rev. D67 (2003), 124012 .

[212] Meyrath, T. P., Schreck, F., Hanssen, J. L., Chuu, C.-S., And Raizen, M. G. Bose-Einstein condensate in a box. Phys. Rev. A 71 (Apr 2005), 041604.

[213] Nauerth, S., Moll, F., Rau, M., Fuchs, C., Horwath, J., Frick, S., And Weinfurter, H. Air-to-ground quantum communication. Nature Photonics 7 (March 31 2013), 382-386.

[214] Nielsen, H. B., And Ninomiya, M. Beta function in a noncovariant YangMills theory. Nucl. Phys. B 141 (1978), 153.

[215] Nielsen, H. B., And Picek, I. Redei like model and testing lorentz invariance. Phys. Lett. B 114 (1982), 141.

[216] Nielsen, H. B., And Picek, I. Lorentz noninvariance. Nucl. Phys. B 211 (1983), 269.

[217] Nielsen, H. B., AND PiceK, I. Lorentz noninvariance. (addendum) On a possible subtraction for the Lorentz noninvariant model. Nucl. Phys. B 242 (1984), 542 .

[218] Obadia, N., And Milgrom, M. On the Unruh effect for general trajectories. Phys. Rev. D 75 (2007), 065006.

[219] Padmanabhan, H., and Padmanabhan, T. Non-relativistic limit of quantum field theory in inertial and non-inertial frames and the principle of equivalence. Phys. Rev. D 84 (2011), 085018.

[220] Padmanabhan, T. Thermodynamical Aspects of Gravity: New insights. Reports in Progress of Physics 73 (2010), 046901.

[221] Padmanabhan, T. Lessons from Classical Gravity about the Quantum Structure of Spacetime. J.Phys. Conf. Ser. 306 (2011), 01200.

[222] Padmanabhan, T. Structural Aspects Of Gravitational Dynamics And The Emergent Perspective Of Gravity. AIP Conf. Proc 1483 (2012), 2120-238. 
[223] Pars, L. A. The Lorentz transformation. Phil. Mag. 42 (1921), 249-258.

[224] Peres, A. Separability Criterion for Density Matrices. Phys. Rev. Lett. 77 (1996), 1413-1415.

[225] Perez, A. Spin foam models for quantum gravity. Classical and Quantum Gravity 20 (Mar. 2003), 43.

[226] Perez, A. Introduction to loop quantum gravity and spin foams. CNUM: C04-06-06.3. ArXiv: 0409061 [gr-qc], 2004.

[227] Pethick, C. J., And Smith, H. Bose-Einstein condensation in dilute gases. Cambridge University Press, 2004.

[228] Plenio, M. B., And Virmani, S. An introduction to entanglement measures. Quant. Inf. Comput. 7 (2005), 1-51.

[229] Polchinski, J. String theory. Vol. 1: An introduction to the bosonic string. Cambridge University Press, 1998.

[230] Polchinski, J. String theory. Vol. 2: Superstring theory and beyond. Cambridge University Press, 1998.

[231] Ponzano, G., And Regge, T. E. Semiclassical limit of Racah coefficients. In Spectroscopic and Group Theoretical Methods in Physics (1968).

[232] Preskill, J. Do black holes destroy information? In Houston 1992, Proceedings, Black holes, membranes, wormholes and superstrings (1992), pp. 22-39.

[233] Regge, T. General relativity without coordinates. Il Nuovo Cimento Series 1019,3 (1961), 558-571.

[234] Rembielinski, J., And Smolinski, K. A. Unphysical predictions of some doubly special relativity theories. Bull. Soc. Sci. Lett. Lodz 53 (2003), 57.

[235] Renner, R. Quantum Information theory, 2009. Lecture notes.

[236] Requardt, M. Emergence of space-time on the Planck scale within the scheme of dynamical cellular networks and random graphs. ArXiv:9612185 [hep-th], 1996.

[237] Rideout, D., Jennewein, T., Amelino-Camelia, G., Demarie, T. F., Higgins, B. L., ET AL. Fundamental quantum optics experiments conceivable with satellites: Reaching relativistic distances and velocities. Class. Quant. Grav. 29 (2012), 224011. 
[238] RindleR, W. Essential Relativity, vol. pp. 51-53. Springer, 2nd ed, New York, 1977.

[239] Robertson, H. Postulate versus observation in the special theory of relativity. Rev. Mod. Phys. 21 (1949), 378.

[240] RovelLI, C. Quantum gravity. Cambridge University Press, 2004.

[241] Rovelli, C. A note on DSR. ArXiv:0808.3505 [gr-qc], 2008.

[242] RovelLI, C. Loop quantum gravity. Living Reviews in Relativity 11, 5 (2008).

[243] Rovelli, C., And Smolin, L. Knot theory and quantum gravity. Phys. Rev. Lett. 61 (Sep 1988), 1155-1158.

[244] Rubino, E., Belgiorno, F., Cacciatori, S., Clerici, M., Gorini, V., ET AL. Experimental evidence of analogue Hawking radiation from ultrashort laser pulse filaments. New Journal of Physics 13 (2011), 085005.

[245] Rudolph, J., And et al. Degenerate quantum gases in microgravity. Microgravity Science and Technology 23 (2011), 287-292.

[246] Sabín, C., White, A., Hackermuller, L., and Fuentes, I. Dynamical phase quantum thermometer for an ultracold Bose-Einstein condensate. ArXiv: 1303.6208 [quant-ph], Mar. 2013.

[247] Sakharov, A. Vacuum quantum fluctuations in curved space and the theory of gravitation. Sov. Phys. Dokl. 12 (1968), 1040-1041.

[248] Sarda, K., Eagleson, S., Caillibot, E., Grant, C., Kekez, D., Pranajaya, F., AND ZeE, R. E. Canadian advanced nanospace experiment 2: Scientific and technological innovation on a three-kilogram satellite. Acta Astronautica 59 (2006), 236 - 245. Space for Inspiration of $\mathrm{Hu}-$ mankind, Selected Proceedings of the 56th International Astronautical Federation Congress, Fukuoka, Japan, 17-21 October 2005 Space for Inspiration of Humankind, Selected Proceedings of the 56th International Astronautical Federation Congress, Fukuoka, Japan, 17-21 October 2005.

[249] SARIDAkis, E. N. Constraining Hořava-Lifshitz gravity from neutrino speed experiments. Gen.Rel.Grav. 45 (2013), 387-394.

[250] SATz, A. Then again, how often does the Unruh-DeWitt detector click if we switch it carefully? Class. Quant. Grav. 24 (2007), 1719. 
[251] Scheidl, T., Wille, E., And Ursin, R. Quantum optics experiments using the International Space Station: a proposal. New Journal of Physics 15, 4 (Apr. 2013), 043008.

[252] Scherk, J., And Schwarz, J. H. Dual models fon nonhadrons. Nucl. Phys. B81 (1974), 118-144.

[253] Schlicht, S. Considerations on the Unruh effect: Causality and regularization. Class. Quant. Grav 21 (2004), 4647.

[254] Schutzhold, R., And UnRuh, W. G. Large-scale nonlocality in 'doubly special relativity' with an energy-dependent speed of light. JETP Lett. 78 (2003), 431.

[255] Severi, F. Aspetti matematici dei legami tra relatività e senso comune. Cinquant'anni di Relatività. Giunti, Firenze, 1955, pages 309-333.

[256] Shannon, C. E. A Mathematical Theory of Communication. Bell System Technical Journal 27, 3 (July/October 1948), 379-423.

[257] Shannon, C. E., And Weaver, W. The Mathematical Theory of Communication. University of Illinois Press, 1949.

[258] Shilov, G. E. Elementary real and complex analysis. Dover, New York, 1996.

[259] Silva, J. F., And Parada, P. A. Shannon entropy convergence results in the countable infinite case. In Proceedings of the 2012 IEEE International Symposium on Information Theory. (2012).

[260] Smolin, L. Classical paradoxes of locality and their possible quantum resolutions in deformed special relativity. Gen. Rel. Grav. 43 (2011), 3671.

[261] Sotiriou, T. P., Visser, M., And Weinfurtner, S. Phenomenologically viable Lorentz-violating quantum gravity. Phys. Rev. Lett. 102 (2009), 251601.

[262] Sotiriou, T. P., Visser, M., And Weinfurtner, S. Quantum gravity without Lorentz invariance. JHEP 0910 (2009), 033.

[263] SRednicki, M. Entropy and area. Phys. Rev. Lett 71 (1993), 666.

[264] Sriramkumar, L., and Padmanabhan, T. Response of finite time particle detectors in non-inertial frames and curved space-time. Class. Quant. Grav 13 (1996), 2061. 
[265] Srivastava, A. M. Invariant speed in special relativity. American Journal of Physics 49 (1981), 504.

[266] Süssmann, G. Begründung der Lorentz-gruppe allein mit symmetrie- und relativitäts-annahmen. Zeitsch. Naturf. $24 A$ (1969), 495-498.

[267] TerletskiI, Y. P. Paradoxes in the theory of relativity. Plenum, New York, 1968.

[268] Terno, D. R. Entropy, holography, and the second Law. Phys. Rev. Lett. 93 (Jul 2004), 051303.

[269] TorRetti, R. Relativity and geometry, vol. pp. 76-82. Dover, New York, 1996.

[270] Unruh, W. Experimental black hole evaporation. Phys. Rev. Lett. 46 (1981), $1351-1353$.

[271] Unruh, W. G. Notes on black hole evaporation. Phys. Rev. D 14 (1976), 870 .

[272] URsin, R., AND et al. Free-space distribution of entanglement and single photons over $144 \mathrm{~km}$. Nature Physics 3 (2007), 481 - 486.

[273] Vidal, G., and Werner, R. F. Computable measure of entanglement. Phys. Rev. A 65, 3 (2002), 032314.

[274] Villoresi, P., AND et al. Experimental verification of the feasibility of a quantum channel between space and Earth. New Journal of Physics 10, 3 (Mar. 2008), 033038.

[275] VisseR, M. Lorentzian wormholes: From Einstein to Hawking. AIP Press, now Springer-Verlag, New York, 1995.

[276] Visser, M. Acoustic black holes: Horizons, ergospheres, and Hawking radiation. Class. Quant. Grav. 15 (1998), 1767-1791.

[277] Visser, M. Sakharov's induced gravity: A Modern perspective. Mod. Phys. Lett. A17 (2002), 977-992.

[278] Visser, M. Essential and inessential features of Hawking radiation. Int. J. Mod. Phys. D 12 (2003), 649. 
[279] Visser, M. Lorentz symmetry breaking as a quantum field theory regulator. Phys. Rev D80 (2009), 025011.

[280] Visser, M. Power-counting renormalizability of generalized Hořava gravity. ArXiv:0912.4757 [hep-th]], 2009.

[281] Visser, M. Status of Hořava gravity: A personal perspective. J. Phys. Conf. Ser. 314 (2011), 012002.

[282] Visser, M. Zipf's law, power laws, and maximum entropy. New Journal of Physics 15 (2013), 043021.

[283] Visser, M., Barceló, C., And Liberati, S. Acoustics in Bose-Einstein condensates as an example of broken Lorentz symmetry. ArXiv:hep-th/0109033, 2001.

[284] Visser, M., and Molina-Paris, C. Acoustic geometry for general relativistic barotropic irrotational fluid flow. New Journal of Physics 12 (2010), 095014 .

[285] von Ignatowsky, W. A. Einige allgemeine Bemerkungen zum Relativitätsprinzip. Verh. Deutsch. Phys. Ges. 12 (1910), 788-796.

[286] von Ignatowsky, W. A. Einige allgemeine Bemerkungen zum Relativitätsprinzip. Phys. Zeitsch. 11 (1910), 972-976.

[287] von Ignatowsky, W. A. Das Relativitätsprinzip. Arch. Math. Phys. 3, (17) (18) (1911), 1-24; 17-41.

[288] von Ignatowsky, W. A. Eine Bemerkung zu meiner Arbeit 'Einige allgemeine Bemerkungen zum Relativitätsprinzip'. Phys. Zeitsch. 12 (1911), 779.

[289] VOn Neumann, J. Mathematische Grundlagen der Quantenmechanik. Springer, Berlin, 1955.

[290] von Neumann, J. Mathematical Foundations of Quantum Mechanics. Princeton University Press, Princeton, 1996.

[291] Wald, R. M. General Relativity. University of Chicago Press, 1984.

[292] WANG, J.-Y., AND et al. Direct and full-scale experimental verifications towards ground-satellite quantum key distribution. Nature Photonics 7 (May 2013), 387-393. 
[293] Wang, P., Wu, H., And YAng, H. Superluminal neutrinos and domain walls. ArXiv: 1109.6930 [hep-ph], 2011.

[294] Watrous, J. Theory of Quantum Information, 2008. Lecture notes.

[295] WeHrL, A. Three theorems about entropy and convergence of density matrices. Reports on Mathematical Physics 10 (1976), 159-163.

[296] Wehrl, A. General properties of entropy. Reviews of Modern Physics 50 (1978).

[297] Weinfurtner, S., Sotiriou, T. P., And Visser, M. Projectable HořavaLifshitz gravity in a nutshell. J. Phys. Conf. Ser. 222 (2010), 012054.

[298] Weinfurtner, S., Tedford, E. W., Penrice, M. C., Unruh, W. G., and Lawrence, G. A. Measurement of stimulated Hawking emission in an analogue system. Phys.Rev.Lett. 106 (2011), 021302.

[299] Wheeler, J. A. Geometrodymanics and the issue of the final state. Relativity, Groups and Topology. Gordon and Breach, 1964.

[300] Wheeler, J. A. Geometrodynamics. Academic Press, New York, 1964.

[301] Whitehead, A. N. An Enquiry Concerning the Principles of Natural Knowledge, vol. chapter XIII. Cambridge University Press, Cambridge, 1919.

[302] Wilson, C. M., Johansson, G., Pourkabirian, A., Simoen, M., Johansson, J. R., Duty, T., Nori, F., And Delsing, P. Observation of the dynamical Casimir effect in a superconducting circuit. Nature 479 (Nov. 2011), 376-379.

[303] Winter, W. Constraints on the interpretation of the superluminal motion of neutrinos at OPERA. Phys.Rev. D85 (2012), 017301.

[304] Wolf, M. M., Verstraete, F., Hastings, M. B., And Cirac, J. I. Area laws in quantum systems: Mutual information and correlations. Phys. Rev. Lett. 100 (2008), 070502.

[305] Yeung, R. W. Information Theory and Network Coding. Springer Verlag, 2002, 2008.

[306] Yin, J., AND et al. Quantum teleportation and entanglement distribution over 100-kilometre free-space channels. Nature 488 (Aug. 2012), 185-188. 